Pedro Manuel González del Foyo

\title{
Verificação Formal de Sistemas Discretos Distribuídos
}

Tese apresentada à Escola Politécnica da Universidade de São Paulo para obtenção do Título de Doutor em Engenharia Mecânica. 



\section{Pedro Manuel González del Foyo}

\section{Verificação Formal de Sistemas Discretos Distribuídos}

Tese apresentada à Escola Politécnica da Universidade de São Paulo para obtenção do Título de Doutor em Engenharia Mecânica.

Área de concentração:

Engenharia de Controle e Automação Mecânica

Orientador:

Prof. Dr. José Reinaldo Silva 
Este exemplar foi revisado e alterado em relação à versão original, sob responsabilidade única do autor e com anuência de seu orientador.

São Paulo, 16 de dezembro de 2009

Assinatura do autor

Assinatura do orientador

\section{Ficha Catalográfica}

González del Foyo, Pedro Manuel

Verificação Formal de Sistemas Discretos Distribuídos. \ P.M. González del Foyo. - ed.rev. - São Paulo, 2009.

160 p.

Tese (Doutorado) - Escola Politécnica da Universidade de São Paulo. Departamento de Engenharia Mecatrônica e de Sistemas Mecânicos.

1. Redes de Petri (modelos) 2. Sistemas Discretos 3. Sistemas Distribuídos I. Universidade de São Paulo. Escola Politécnica. Departamento de Engenharia Mecatrônica e de Sistemas Mecânicos. II. t. 


\section{Agradecimentos}

Agradeço ao orientador deste trabalho, Prof. Doutor José Reinaldo Silva pelo suporte dado na realização deste trabalho e pelas continuas discussões que tanto aportaram a melhoria do mesmo.

Agradeço também aos colegas do Dlab pela sua contribuição a manter um ambiente amigável de trabalho. Agradeço especialmente aos colegas José Armando San Pedro Miralles e Arianna Zoila Olivera Sálmon pela sua ajuda na implementação da rede GHENeSys assim como do verificador de modelos.

A minha familia, pela sua ajuda e compreensão, especialmente a meus pais, pela formação que me deram, e o seu incentivo na procura constante de superação profissional.

Finalmente gostaria de agradecer aos professores do Departamento de Mecatrônica e de Sistemas Mecânicos, especialmente ao Prof. Doutor Newton Maruyama, que de alguma forma contribuíram na minha formação e à conclusão deste trabalho. 



\section{Resumo}

O presente trabalho trata da verificação e design de sistemas complexos, especificamente da verificação de sistemas de tempo real concorrentes e distribuídos. Propõe-se uma técnica enumerativa para a verificação formal de modelos que permite determinar a validade de propriedades quantitativas, além das qualitativas. A técnica proposta separa a construção do espaço de estados dos algoritmos de rotulação das fórmulas temporais, o que possibilita a diminuição da complexidade do processo de verificação, tornando-o viável para aplicações práticas. A técnica proposta foi inicialmente aplicada sobre modelos de redes de Petri temporizadas e depois em uma rede unificada chamada GHENeSys para aproveitar as características de abstração, hierarquia e de elementos de interação chamados pseudo-boxes. A definição da rede GHENeSys foi modificada para permitir a modelagem de sistemas onde os requisitos temporais devem ser expressos através de atrasos e prazos como é o caso dos sistemas de tempo real. A rede suporta ainda mecanismos de refinamento tanto para os elementos ativos quanto os passivos. A demonstração da manutenção de propriedades como invariantes, vivacidade, limitação assim como da validade de fórmulas lógicas no processo de refinamento constitui um aspecto fundamental no projeto de sistemas complexos, e foi portanto revista em detalhes para a rede GHENeSys. Alguns exemplos práticos são apresentados para avaliar o desempenho dos algoritmos e um estudo de caso finaliza a apresentação, onde se pode contrastar os algoritmos propostos com os implementados na ferramenta UPPAAL.

Palavras-chave: Verificação formal. Verificação de modelos. Redes de Petri temporizadas. Sistemas discretos. 



\section{Abstract}

This work deals with the process of design and verification of complex systems, mainly real time, concurrent and distributed systems. An enumerative technique is proposed for model-checking which is capable of determining both quantitative and qualitative properties. The proposed technique detach the algorithm for labeling the formula being checked from the state space construction, allowing a better result in the verification process. This model-checking approach shows itself valuable in practical applications. This approach was first applied to systems modeled by Time Petri Nets and further extended to a unified net called GHENeSys, which includes abstraction and hierarchy concepts as well as elements for data and control interchange, called pseudo-boxes. The GHENeSys definition was modified in order to deal with systems in which temporal requirements can be expressed through delays and deadlines as in the real-time systems. The GHENeSys environment supports a refinement technique applied to both passive and active elements. Net properties like invariants, liveness, boundedness and also the validity of temporal formulas was proved to be maintained through the refinement process if some conditions are satisfied. Such characteristics are useful to deal with complex systems design. Some experiments based on well known academic articles were used to avaliate the performance of the algorithms and a case study is presented in order to compare obtained results with those obtained using the UPPAAL tool.

Keywords: Formal Verification. Model-checking. Time Petri nets. Discrete Event Systems. 



\section{Lista de Figuras}

2.1 Exemplo de rede de autômatos temporizados para modelar o problema de passagem de nível. . . . . . . . . . . . . . . . . . . . . 13

2.2 Exemplos de árvores binárias de decisão. . . . . . . . . . . . . . 18

3.1 Propriedades $(\mathrm{EE}),(\mathrm{EA})$ e $(\mathrm{AE}) \ldots \ldots \ldots$

3.2 Rede de Petri temporizada e seus grafos de classes de estados lineares e atômicas . . . . . . . . . . . . . . . . . . . 41

3.3 Grafos de classes de estados lineares e atômicas para o exemplo da figura $3.2 \ldots \ldots \ldots 4 \ldots$. . . . . . . . . . . . . . . . . . . . 42

3.4 Exemplo de RPT modelando um sistema com concorrência, conflito e sincronização. . . . . . . . . . . . . . . . . . . . . 44

3.5 Árvore de atingibilidade com seus respectivos intervalos de tempo

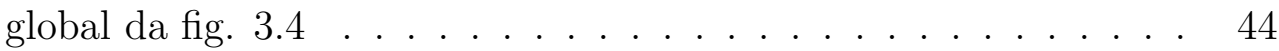

3.6 LTS construído a partir das classes de estados lineares usando TINA 45

3.7 Carta de tempo da RPT da figura $3.2 \ldots \ldots$. . . . . . . . . . 47

3.8 Grafo das classes de estados com restrições da RPT na figura 3.2 . 58

4.1 Exemplo de rede GHENeSys . . . . . . . . . . . . . . . . . . . . 92

4.2 Novo Diagrama de Classes da rede GHENeSys . . . . . . . . . . 113

5.1 Exemplo de workflow . . . . . . . . . . . . . . 116

5.2 Modelo em RPT do exemplo de workflow da figura 5.1 . . . . . 116

5.3 Resultados da verificação do exemplo da figura 5.1, ressaltando em que estados a fórmula $\forall \diamond_{\leq 25} p 11$ é verdadeira . . . . . . . . . . 118

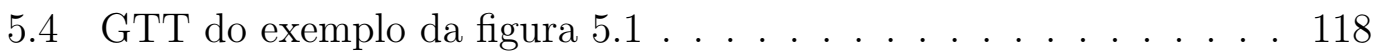

5.5 Estados onde a fórmula $\forall \diamond_{\leq 5}(p 1 \wedge p 2 \wedge p 3)$ é verdadeira . . . . 119

5.6 Sistema de evaporação. Malha fechada do sistema composto pelo controlador e a planta. . . . . . . . . . . . . . . . . 120 
5.7 Modelo do Evaporador . . . . . . . . . . . . . . . . . . 122

5.8 Modelo do controlador . . . . . . . . . . . . . . . . . . 123

5.9 Resultado do computo das classes de estados com restrições para o sistema de evaporação . . . . . . . . . . . . . . . . . . . . 124

5.10 Resultado da verificação das propriedades de segurança para o sistema de evaporação . . . . . . . . . . . . . . . . . . . . 125

5.11 Resultado da verificação das propriedades de vivacidade para o sistema de evaporação . . . . . . . . . . . . . . . . . . 125

5.12 Exemplos de rede de Petri temporizadas usadas nos experimentos de Yoneda e Ryuba (1998) . . . . . . . . . . . . . . . . 126

5.13 Exemplo de modelagem que conduz a erro na verificação de propriedades . . . . . . . . . . . . . . . . . 129

C.1 Modelo em UPPAAL dos módulos Tank1, Tank2, Heater, Mixer, Steam e Condenser. . . . . . . . . . . . . . . . . 157

C.2 Modelo em UPPAAL dos módulos Liquid, Alarm, Ton_3, Ton_4 e Ton_6. . . . . . . . . . . . . . . . . . . . . . 158

C.3 Especificação em SFC do controlador do Sistema de Evaporação. . 158

C.4 Modelo em UPPAAL do módulo Ladder. . . . . . . . . . . . . . 159

C.5 Resultado da verificação para o Sistema de Evaporação. . . . . . . 160 


\section{Lista de Algoritmos}

Algoritmo 3.1 Verificação de fórmulas TCTL em todos os estados do GTT 67

Algoritmo 3.2 Subrotina AUcheck . . . . . . . . . . . . . . . . . 69

Algoritmo 3.3 Busca em profundidade para o operador UNTIL com quantificador universal . . . . . . . . . . . . . 70

Algoritmo 3.4 Subrotina TcheckB usada pela subrotina DFS . . . . . . . 71

Algoritmo 3.5 Subrotina UpdateList . . . . . . . . . . . . . . . . . 72

Algoritmo 3.6 Subrotina EUcheck . . . . . . . . . . . . . . . . 73

Algoritmo 3.7 Busca em profundidade para o operador UNTIL com quantificador existencial . . . . . . . . . . . . . . . . 74

Algoritmo 3.8 Subrotina TcheckB usada pela subrotina DFSeu . . . . . . 75

Algoritmo 3.9 Determinação da diferença de tempo entre dois conjuntos de estados . . . . . . . . . . . . . . 76

Algoritmo 3.10 Subrotina para manipular as listas f . . . . . . . . . . 78

Algoritmo 3.11 Verificação de sub-fórmulas de estados para o problema da verificação local . . . . . . . . . . . . . . . . . . . . 79

Algoritmo 3.12 Verificação de fórmulas TCTL em um estado específico do GTT ..................... 80

Algoritmo 3.13 Subrotina para verificar fórmulas com o operador UNTIL com quantificador universal . . . . . . . . . . . . . 81

Algoritmo 3.14 Subrotina que procura um contra-exemplo a partir de um determinado estado . . . . . . . . . . . . . . 82

Algoritmo 3.15 Subrotina para verificar fórmulas com o operador UNTIL com quantificador existencial . . . . . . . . . . 83

Algoritmo 3.16 Subrotina que procura um avalista a partir de um determinado estado . . . . . . . . . . . . . . . 84 
Algoritmo 4.1 Determinação do tempo mínimo de habilitação das atividades habilitadas num determinado estado . . . . . . . . 96 


\section{Lista de Tabelas}

3.1 Algumas abreviaturas de formulas TCTL . . . . . . . . . . . . . 32

3.2 Semântica do operador de diferença temporal . . . . . . . . . . . 35

3.3 Restrição usada na divisão da classe $s_{i}$ para a condição 3.4 . . . . 52

3.4 Restrição usada na divisão da classe $s_{i}$ para a condição 3.5 . . . . 52

3.5 Classes de estado com restrições para o exemplo da figura 3.2 . . 57

3.6 Requisito de espaço de memória em bits dos algoritmos de verificação global e local. . . . . . . . . . . . . . . . . . . . . 88

4.1 Restrição usada na divisão da classe $s_{i}$ para a condição 4.10 . . 106

4.2 Restrição usada na divisão da classe $s_{i}$ para a condição 4.11 . . . 106

5.1 Especificação em TCTL do exemplo de workflow . . . . . . . . . . 117

5.2 Sinais do controlador presentes no modelo do evaporador . . . . . 122

5.3 Sinais dos sensores do processo presentes no modelo do controlador 122

5.4 Especificação em TCTL do funcionamento do sistema de evaporação124

5.5 Resultados dos experimentos de Yoneda e Ryuba . . . . . . . . . 127

A.1 Classes de estado lineares obtidas usando a opção -W para o exem-

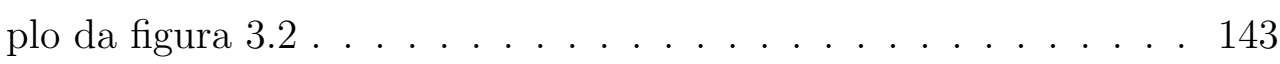

A.2 Classes de estado atômicas obtidas usando a opção -A para o exemplo da figura $3.2 \ldots$. . . . . . . . . . . . . . . . . . . 144

A.1 Algumas classes de complexidades presentes em problemas de verificação. . . . . . . . . . . . . . . . . . . . . 153

B.1 Tempo de execução e requerimento de espaço de memória para algumas implementações de verificadores de modelos . . . . . . 155

B.2 Requerimento de espaço de memória em bits para verificação da lógica CTL . . . . . . . . . . . . . . . . 156 
B.3 Requerimento de espaço de memória em bits para armazenar a estrutura do grafo . . . . . . . . . . . . . . . . . . 156 


\section{Lista de Abreviaturas}

CPU Central Processing Unit

VLSI Very Large Scale Integration

TPTL Timed Propositional Temporal Logic

TCTL Timed Computation Tree Logic

MTL Metric Temporal Logic

CSP Communicating Sequential Processes

CCS Calculus of Communicating Systems

TCSP Timed Communicating Sequential Processes

PTL Linear-time Propositional Temporal Logic

LTL Linear-time Temporal Logic

TLTL Timed Linear-time Temporal Logic

RTL Real Time Logic

LPTL Linear-time Propositional Temporal Logic

APTL Asynchronous Propositional Temporal Logic

CTL Computation Tree Logic

RTCTL Real Time Computation Tree Logic

ACP Algebra of Communicating Processes

UML Unified Modeling Language

UML-RT Unified Modeling Language for Real Time

RPTs Redes de Petri Temporizadas

BDD Binary Decision Diagram 
DBM Difference Bounded Matrix

NDD Numerical Decision Diagram

S-TPNs Scheduling-Time Petri Nets

ICTL Integrator Computation Tree Logic

ITCPN Interval Timed Coloured Petri Nets

TNL Temporal Nets Logic

ALMC Algorithm for Local Model Checking

TMPN Timed Modular Petri Net

EFT Earliest Firing Time

LFT Latest Firing Time

WFMS WorkFlows Management System

CSZ Concrete State Zone

CSZG Concrete State Zone Graph

CC-CSZG Convex combination Contracted Concrete State Zone Graph

SSCG Strong State Class Graph

CSCG Constrained State Class Graph

CLP Controlador Lógico Programavel 


\section{Lista de Símbolos}

Na notação das fórmulas, as seguintes convenções foram utilizadas:

- letras maiúsculas são conjuntos ou matrices, exemplo: $P, A$;

- letras minúsculas são elementos de conjunto, exemplo: $s \in S, t \in \operatorname{enb}(M)$;

- subscrito diferencia um elemento de um conjunto de outro, exemplo: $a_{j}, t_{i}$;

- letras maiúsculas seguidas de minúsculas entre parênteses identificam elementos dentro de uma matriz, exemplo: $D_{t t^{\prime}}, M(p), K(s)$;

- $A \times B$ é o produto cartesiano dos conjuntos $A$ e $B$;

- $|S|$ cardinalidade de $S$ (o número de elementos do conjunto $S$ ); 


\section{Símbolos lógicos e matemáticos}

$N \quad$ Conjunto dos números naturais

$N^{+} \quad$ Conjunto dos números naturais sem o zero

$Q^{+} \quad$ Conjunto dos números racionais positivos

$Q^{+} \cup\{\infty\}$ Conjunto dos números racionais positivos incluindo infinito

$n, m, i, j \quad$ Números naturais quaisquer

$+\quad$ Adição

- Subtração

* Multiplicação escalar

| Tal que

$\wedge \quad$ "e" lógico

$\vee \quad$ "ou" lógico

$\cup \quad$ União entre conjuntos

$\cap \quad$ Intersecção entre conjuntos

$\backslash$ Diferença entre conjuntos

$\emptyset \quad$ Conjunto vazio

$\neq \quad$ Diferente

$\geq \quad$ Maior ou igual que

$\leq \quad$ Menor ou igual que

$\cong \quad$ Relação de equivalência

$\lceil\lambda\rceil \quad$ Conjunto de seqüencias admissíveis por uma rede

$\sigma, \omega \quad$ Seqüência de disparo, caminho numa estrutura tipo árvore

$\omega(t) \quad$ tempo decorrido no caminho $\omega$

$\sigma^{*} \quad$ Permutações possíveis numa seqüência ou caminho

$f, \phi, \phi_{1} \quad$ fórmulas lógicas temporais

$\diamond, \square, U \quad$ operadores modais na lógica temporal

$s \models \phi \quad$ relação de satisfatibilidade, $\phi$ é satisfeita em $s$ ou num caminho que começa em $s$

$s \not \models \phi \quad \phi$ não é satisfeita em $s$ ou num caminho que começa em $s$

$\Omega \quad$ Partição do espaço de estados, Conjunto de classes de estados

EFT $(t) \quad$ Earliest Firing Time da transição $t$

LFT $(t) \quad$ Latest Firing Time da transição $t$

$\downarrow \quad$ operador que devolve o limite inferior de um intervalo

$\uparrow \quad$ operador que devolve o limite superior de um intervalo

$\forall \quad$ Qualquer, para todos

$\exists \quad$ Existe

$\sum \quad$ Somatória 


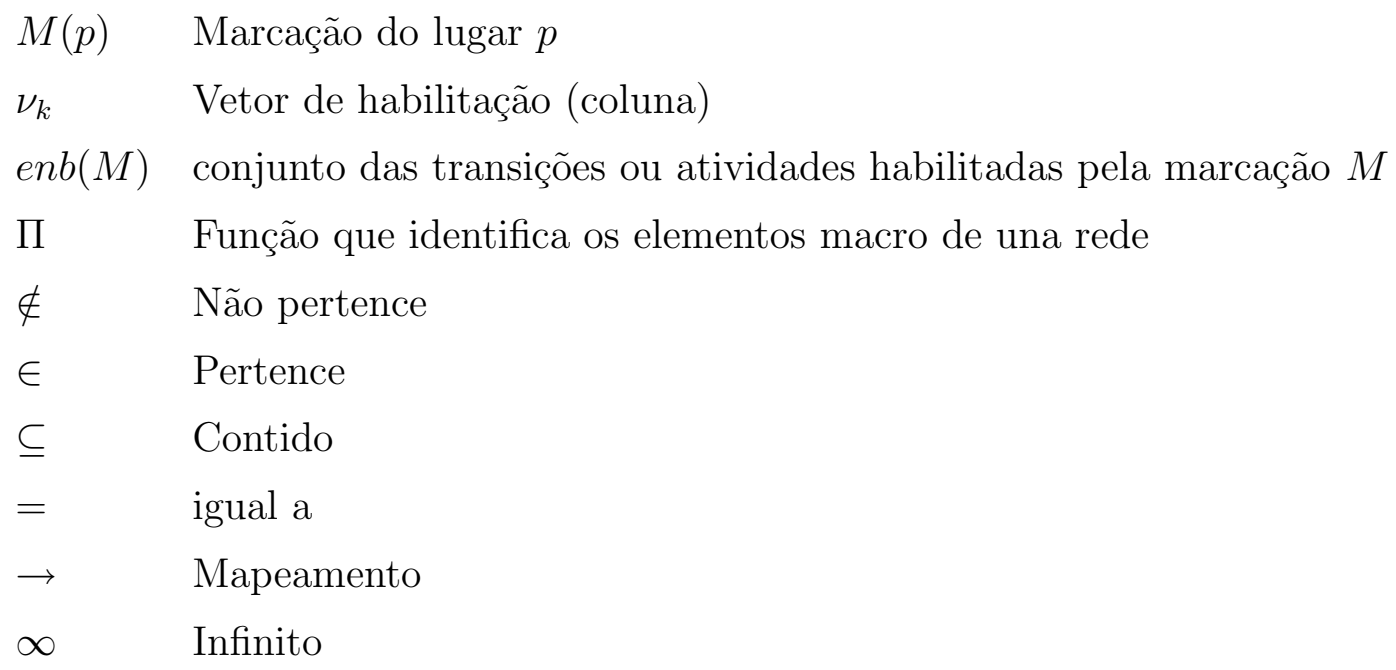

\section{Demais símbolos}
$s \stackrel{t, I(t)}{\longrightarrow} s^{\prime}$ transição do estado $s$ ao estado $s^{\prime}$ através do disparo da transição $t$ num tempo dentro de intervalo $I(t)$
$C \quad$ Matriz de incidência
$C^{T} \quad$ Transposta da matriz $C$
$x^{\bullet} \quad$ Pós-elementos do elemento $x$
- $x \quad$ Pré-elementos do elemento $x$ 



\section{Sumário}

1 Introdução 1

1.1 Contribuição da tese . . . . . . . . . . . . . . . . . . 3

1.2 Organização do trabalho . . . . . . . . . . . . . . . . 4

2 O Problema da Verificação de Modelos 5

2.1 Abordagens para verificação formal . . . . . . . . . . . 5

2.2 Abordagens para os modelos temporais . . . . . . . . . . 7

2.3 Linguagens para especificação de sistemas . . . . . . . . . . . . . 9

2.4 Formalismos para descrição de sistemas . . . . . . . . . . . . . . . 11

2.4.1 Autômatos híbridos . . . . . . . . . . . . . . . . . 12

2.4.2 Autômatos temporizados . . . . . . . . . . . . . . . 12

2.4.3 Redes de Petri . . . . . . . . . . . . . . . . . . 13

2.4.4 Linguagens gráficas . . . . . . . . . . . . . . . . . 14

2.5 Construção do espaço de estados . . . . . . . . . . . . . . . . 15

2.6 Estruturas para representação do espaço de estados . . . . . . . . 18

2.7 Ambientes Computacionais para Verificação . . . . . . . . . . . 19

2.8 Trabalhos relacionados . . . . . . . . . . . . . . . . . . 21

2.9 Resumo e comentários finais . . . . . . . . . . . . . . . . . 23

3 Uma Abordagem Baseada em Redes de Petri para a Verificação $\begin{array}{ll}\text { de Propriedades Quantitativas } & 27\end{array}$

3.1 As redes de Petri temporizadas . . . . . . . . . . . . . . 29

3.2 A lógica TCTL . . . . . . . . . . . . . . . . . 30

3.2.1 Estendendo a TCTL . . . . . . . . . . . . . . . . 33 
3.3 Um modelo finito para representar sistemas com tempo . . . . . . 35

3.3.1 As classes de estado atômicas . . . . . . . . . . . . . 39

3.3.2 As classes de estados com restrições . . . . . . . . . . . . 47

3.3.3 O grafo das transições temporizadas . . . . . . . . . . . 57

3.3.4 A verificação da TCTL sobre o grafo das transições temporizadas ..................... 60

$3.4 \mathrm{O}$ verificador de modelos . . . . . . . . . . . . . . . 61

3.4.1 Preparando o processo de verificação . . . . . . . . . . 65

3.4.2 A verificação de modelos de forma global . . . . . . . . 67

3.4.3 A verificação de modelos de forma local . . . . . . . . . . 78

3.4.4 Análise dos requisitos de tempo e espaço . . . . . . . . . 84

$4 \quad$ A rede GHENeSys $\quad 89$

4.1 O tempo na rede GHENeSys . . . . . . . . . . . . . . . . 90

4.1.1 Estado e transição de estado . . . . . . . . . . . . . . . 92

4.1.2 A abordagem de classes de estados em GHENeSys . . . . 98

4.2 Abordagem modular do processo de verificação em GHENeSys 107

4.3 Modificação no diagrama de classes da rede GHENeSys . . . . . 112

$\begin{array}{lll}5 & \text { Aplicações e Estudo de Caso } & 115\end{array}$

5.1 Escalonamento de workflows com restrições de tempo . . . . . . 115

5.2 Verificação de sistemas com tempo . . . . . . . . . . . . . . . . 119

5.2.1 Descrição do comportamento do estudo de caso ..... 119

5.2.2 Modelando o sistema usando GHENeSys . . . . . . . . . 121

5.2.3 Formalizando a especificação usando a TCTL . . . . . . . 123

5.2.4 Resultados do processo de verificação formal . . . . . . . . 124

5.3 Comparação dos algoritmos de geração das classes de estados . . . 126

5.4 Comparação dos métodos para verificação formal de sistemas . . . 128 
6.1 Conclusões . . . . . . . . . . . . . . . . . . . . . . . . 131

6.2 Trabalhos Futuros . . . . . . . . . . . . . . . . 133

Referências

Apêndice A - Resultados da ferramenta TINA

A.1 Cálculo dos intervalos de tempo para o grafo das classes de estado atômicas . . . . . . . . . . . . . . . . . . . 144

Apêndice B - Provas

B.1 Prova de correção do algoritmo AUcheck . . . . . . . . . . . . . 147

B.2 Prova da proposição $39 \ldots$. . . . . . . . . . . . . . . . 151

Anexo A - Complexidade de Algoritmos

Anexo B - Complexidade de Algoritmos usados em Verificadores de Modelos CTL

Anexo C - Modelagem do Sistema de Evaporação usando a Ferramenta UPPAAL 



\section{Introdução}

Desde o final do século passado, a busca por métodos formais para o design de sistemas tem se tornado cada vez mais freqüente impulsionada pela possibilidade de implementação de sistemas cada vez mais complexos. A possibilidade de reutilização de módulos, a repetitibilidade da solução, a facilidade de análise e verificação de sistemas de grande porte e a facilidade para a geração da documentação estão entre as razões que justificam a busca por tais métodos, que contribuem para acelerar o processo de design, além de torná-lo mais seguro.

Estes métodos adquirem maior relevância na medida em que sistemas maiores e mais complexos (SILVA; PESSOA, 1992) precisam ser projetados e construídos. Por outro lado, a integração entre as diferentes partes constituintes, ou subsistemas, contribui para o aumento da complexidade. Por outro lado, a automação destes sistemas complexos via o uso de computadores (ou microprocessadores), permite que estes sistemas sejam classificados e modelados como sistemas discretos, tendo em conta o tamanho e a natureza dos dispositivos de medição e atuação.

$\mathrm{Na}$ verdade, boa parte dos sistemas de grande porte são sistemas híbridos que podem ser representados pela evolução de uma mistura de sinais de natureza continua e discreta. Ainda assim, quando sistemas complexos precisam ser projetados é comum olhar o sistema todo como um sistema discreto e, na medida que seja necessário, inserir a parte continua no modelo - por exemplo, em métodos de um objeto (DAVID; ALLA, 2001), (DAVID; ALLA, 2005).

Este trabalho trata sobre sistemas discretos e distribuídos. O termo distribuído faz alusão aos sistemas em que a parte "inteligente" está espalhada por todo o sistema, comunicando-se através de mensagens para garantir suas funcionalidades. Tais sistemas envolvem uma ampla gama de aplicações, desde sistemas embarcados de tempo real até instalações para manufatura em grande escala, incluindo o controle aeroespacial e até controle de tráfego.

A despeito do uso bastante difundido, tanto na academia quanto no mer- 
cado, existem dois problemas básicos que atraem a atenção de pesquisadores em verificação formal: a estabilidade e eficiência dos algoritmos, e a integração do processo de verificação formal com os métodos de design. No Laboratório de Design de Sistemas Automatizados (D-Lab) a abordagem para este problema tem sido direcionada pelo uso de redes de Petri (SILVA; MIYAGI, 1995), (SILVA; MIYAGI, 1996), (SILVA, 1998), e mais recentemente de redes unificadas (SILVA et al., 2009). A rede GHENeSys (General Hierarchical Enhanced Net System) tem se colocado como um dos primeiros ambientes de design baseado em redes unificadas, e um dos objetivos do laboratório é estender o uso destas redes para modelar problemas com tempo. Neste trabalho trataremos do problema da verificação tanto nas redes convencionais como na rede GHENeSys, como uma contribuição à evolução deste sistema para inclusão de model checking.

Na abordagem seguida neste trabalho separaremos o algoritmo de verificação em duas partes: a geração das classes de estado e o rotulado das fórmulas. Esta separação é em si uma contribuição, dado que o espaço de estados - que é responsável pela explosão combinatória dos algoritmos - é feita somente uma vez potencializando a verificação de propriedades em seguida. O processo enumerativo é escolhido como forma de explorar ao máximo todas as possibilidades de análise, uma vez geradas as classes de estado. O objetivo final é poder verificar propriedades quantitativas, além da análise qualitativa feita pelos sistemas disponíveis na literatura.

A aplicação do método na rede GHENeSys, além de explorar a integração do método de design baseado em redes de Petri tem ainda a vantagem de reduzir a aplicação dos algoritmos propostos para sistemas distribuídos a um processo semelhante a um sistema único conectado com o que chamamos de pseudo-boxes. Estes lugares de marcação persistente na verdade denotam a ligação de um subsistema com os demais, trocando sinais e informação via os pseudo-boxes. Portanto cada sub-sistema pode ser analisado no seu contexto próprio, isto é, submetido ao processo de integração com os demais sub-sistemas via pseudo-box. O colapso destes pseudo-boxes para geração do modelo distribuído geral é absolutamente desnecessário do ponto de vista da verificação de propriedades.

Estudos de caso serão direcionados para aplicações em workflow e em sistemas industriais. Entretanto vale ressaltar o uso potencial dos algoritmos aqui propostos para sistemas embarcados e sistemas de tempo real em geral. Esta aplicação potencial será discutida em alguns pontos do texto. Finalmente outra aplicação potencial importante é na área de scheduling, isto é, nos sistemas de planejamento com recurso e tempo. Este tópico é objeto de outros traba- 
lhos, também do D-Lab, e se reflete na proposição de outro sistema chamado itSIMPLE (Integrated Tools and Software Interface to the Modeling of Planning Systems)(VAQUERO et al., 2007). No futuro a proposta seria integrar este sistema com o sistema GHENeSys.

Os resultados obtidos neste trabalho apontam para uma utilização melhor dos métodos enumerativos. Estes resultados são comparados com algoritmos bem conhecidos para a verificação de propriedades quantitativas e, para referência, com outros algoritmos conhecidos para análise e verificação qualitativa. Não é o caso de se fazer uma comparação exaustiva com os ambientes e sua integração com o processo de design, até porque a existência de poucas abordagens para verificação tanto qualitativa quanto quantitativa não justifica esta comparação. É bem mais efetiva a abertura para esta possibilidade futura, mostrando que o algoritmo aqui apresentado justifica o investimento em sistemas que incluam a verificação como parte do processo de projeto, em particular sistemas para suporte a processos distribuídos.

Sintetizaremos a seguir as principais contribuições desta tese.

\subsection{Contribuição da tese}

Entre as maiores contribuições que esperamos apresentar neste trabalho podemos citar as seguintes:

- Uma abordagem para a verificação de propriedades quantitativas e qualitativas que melhora a performance das já existentes.

- Uma nova condição suficiente para que uma partição de classes de estados preservem as propriedades de tempo ramificado que permite a geração de partições mais eficientes.

- Demonstração das condições necessárias e suficientes para que a estrutura proposta reflita corretamente o comportamento temporal do modelo.

- Algoritmos para verificar os modelos global e localmente.

- Aplicação de técnicas de abstração para diminuir a complexidade do processo de verificação. 


\subsection{Organização do trabalho}

O presente trabalho está organizado da seguinte forma:

No Capítulo 2 apresenta-se uma revisão sobre diferentes técnicas de verificação formal dando ênfase na verificação de modelos. Os diferentes aspectos envolvidos na verificação de modelos (estruturas, técnicas de verificação, modelos temporais, linguagens de especificação, etc.) são analisados de forma detalhada. Trabalhos relacionados são abordados de forma crítica e explicitadas as diferenças com a presente proposta.

O Capítulo 3 introduz a proposta de um método de verificação de modelos que usa uma estrutura onde a verificação da lógica TCTL pode ser executada em tempo polinomial. Neste capítulo apresentam-se os argumentos que sustentam as escolhas dos diferentes aspectos envolvidos na verificação de sistemas com tempo.

O Capítulo 4 apresenta as modificações propostas a rede GHENeSys para permitir a modelagem, análise e verificação de sistemas com tempo. Finalmente mostramos como diminuir a complexidade do processo de design destes sistemas aplicando técnicas de abstração e estruturação já existentes na rede GHENeSys.

O Capítulo 5 apresenta diferentes áreas de aplicação para a técnica de verificação proposta, mostrando através de exemplos como isto pode ser feito. Também discutem-se os resultados obtidos em um estudo de caso, tanto usando a técnica proposta como outras técnicas disponíveis na literatura.

Finalmente o Capítulo 6 apresenta as conclusões deste trabalho assim como algumas proposta para a continuidade do mesmo. 


\section{O Problema da Verificação de Modelos}

\subsection{Abordagens para verificação formal}

No campo da verificação formal existem quatro abordagens fundamentais:

Verificação de satisfabilidade: Nesta abordagem tanto a descrição do sistema $(D)$ quanto as especificações $(E)$ para o seu comportamento são representadas por fórmulas lógicas. Assim, a verificação consiste em provar que $D \rightarrow E$ é um teorema ou que $D \wedge \neg E$ é uma contradição.

Verificação de modelos: Nesta abordagem a descrição do sistema é feita através de um autômato e as especificações são representadas por fórmulas lógicas. O problema é então verificar se o modelo do autômato satisfaz a cada uma das fórmulas.

Simulação: Nesse caso tanto a descrição do sistema $(D)$ quanto as especificações $(E)$ são representadas por sistemas estado-transição (autômatos) e verificase se cada comportamento de $(D)$ é um comportamento de $(E)$.

Prova de teoremas: Nesse caso tanto o comportamento do sistema quanto as propriedades desejadas são dadas por fórmulas em numa determinada representação formal. Esta representação é composta por um conjunto de axiomas e regras de inferência. A verificação consiste em demonstrar a propriedade (proposição) desejada a partir dos axiomas.

Entre estas abordagens a verificação de modelos tem sido a mais utilizada na literatura acadêmica da área de verificação formal. Algumas razões para isto são:

- O processo de verificação é totalmente automático, o que limita a possibilidade de ocorrência de erros. 
- Existem várias lógicas temporais e não temporais com suficiente expressividade para especificar sistemas reais para as quais o problema da verificação é decidível.

- Em geral, a complexidade dos problemas de verificação de modelos é menor que a do problema de satisfabilidade. Por exemplo, para a lógica temporal TCTL o problema de satisfabilidade é indecidível enquanto o problema de verificação de modelos é PSPACE-completo (ALUR; COURCOUBETIS; DILL, $1993)^{1}$.

- Os algoritmos de verificação de modelos produzem contra-exemplos que ajudam a identificar e corrigir erros de design.

A primeira abordagem para verificação automática de $\operatorname{modelos}^{2}$ foi apresentada por Clarke e Emerson (CLARKE; EMERSON, 1981),(EMERSON; CLARKE, 1980) no começos dos anos 80, motivando o desenvolvimento de outras contribuições à pesquisas de mecanismos de verificação. Nestes trabalhos nota-se que a maior limitação prática ao bom desempenho dos algoritmos de verificação de modelos é devido ao tamanho do espaço de estados, que cresce exponencialmente com o número de componentes independentes do sistema (HENZINGER et al., 1992).

O desafio da verificação de modelos desde os trabalhos pioneiros de Clarke e Emerson é obter algoritmos de verificação eficientes, sem afetar a expressividade dos formalismos usados na descrição e especificação do comportamento do sistema. Neste sentido, resultados expressivos têm sido encontrados nas áreas de verificação de software e de sistemas VLSI. Em (CLARKE et al., 1996) foi publicado um resumo de projetos que resultaram bem sucedidos nestas áreas.

Entretanto, o mesmo sucesso não se estendeu para os sistemas dependentes do tempo, especificamente os de tempo real. Apesar de vários resultados terem sido obtidos na verificação de sistemas de tempo real, e do sucesso de várias ferramentas e ambientes computacionais criados para verificação, a verificação de modelos temporizados está aquém das necessidades das aplicações atuais. A razão para isso é o aumento da complexidade dos processos de verificação com a introdução do tempo como variável independente.

Lamport (1977) classificou propriedades temporais em dois grupos: propriedades de segurança (safety properties) e propriedades de vivacidade (liveness

\footnotetext{
${ }^{1}$ Veja no Anexo A uma breve descrição sobre complexidade e a denominação usada para representar complexidade de algoritmos

${ }^{2}$ Para fazer a verificação de um determinado sistema de forma automática, este precisa ser modelado com um número de estados finito.
} 
properties). Intuitivamente, as propriedades de vivacidade garantem que "algo bom" possivelmente acontecerá, e as propriedades de segurança garantem que "algo ruim" nunca deve acontecer.

Evidentemente seria desejável que os verificadores de modelos pudessem verificar todo tipo de propriedades, mas nem sempre isto é possível ou mesmo viável. A escolha da linguagem para especificação do sistema e dos algoritmos de verificação deve ser orientada pela expressividade (quais propriedades podem ser especificadas) e complexidade (a dificuldade para verificar essas propriedades) geralmente é possível projetar algoritmos mais eficientes para lógicas com expressividade limitada. Por outro lado, o problema da verificação de modelos pode ser abordado tanto global como localmente. A verificação global determina a validade da fórmula em todos os estados da estrutura usada para definir o comportamento do sistema modelado, enquanto a verificação local só determina a validade da fórmula no estado solicitado.

A seguir abordaremos os tópicos fundamentais da pesquisa sobre verificação de modelos os quais esclarecerão os critérios de escolha usados na tese.

\subsection{Abordagens para os modelos temporais}

Entre os diversos esquemas de verificação de modelos existentes destacam-se aqueles que têm o seu comportamento fortemente dependente do tempo. Pare estes existem esquemas de verificação que usam diferentes abordagens para modelos temporais. Basicamente, as três abordagens básicas para modelar sistemas com tempo são:

Tempo discreto: Onde se usa o domínio dos números inteiros para indexar o instante de tempo em que o valor das variáveis características do sistema é lido. Esta abordagem requer a escolha de um "tempo de amostragem" (time-step) fixo para modelar a passagem do tempo, o que limita a precisão com que o sistema pode ser modelado. Em teoria, erros podem passar desapercebidos na verificação por causa desta aproximação. Entretanto, na prática, se escolhemos valores suficientemente pequenos para a amostragem do tempo, o espaço de estados do sistema pode crescer tanto que torna a verificação impossível de ser realizada.

Relógio fictício: Esta abordagem introduz uma transição especial no modelo. O tempo é representado no domínio dos números naturais e é incrementado em uma unidade cada vez que a transição especifica é disparada. O tempo 
transcorrido entre dois eventos é medido através do número de vezes que a transição foi disparada. Nesta abordagem só é possível determinar restrições de tempo com uma margem de tolerância de dois segundos. Esta abordagem, ainda que pareça imprecisa (e de fato o é), pode ser usada com sucesso em determinados tipo de sistemas como, por exemplo, no projeto de circuitos lógicos onde a passagem de tempo é medida em ticks de relógios.

Tempo denso: Neste caso o tempo é modelado no domínio dos números reais. Por causa disso, o intervalo entre eventos pode ser determinado de forma precisa.

As abordagens de tempo discreto, como por exemplo as lógicas TPTL e MTL (ALUR; HENZINGER, 1990), são adequadas para sistemas onde todos os processos concorrentes compartilham o mesmo relógio enquanto as abordagens de tempo denso são melhores para sistemas distribuídos com múltiplos relógios e temporizadores (WANG, 2004). A lógica TCTL (ALUR; COURCOUBETIS; DILL, 1993) é um exemplo do uso da abordagem de tempo denso.

A escolha do tipo de modelo temporal a ser usado no esquema de verificação pode afetar a complexidade do processo. Um exemplo disso é a lógica TPTL. Enquanto a complexidade da TPTL usando tempo discreto é EXPSPACE, usando tempo denso ela se torna indecidível.

Intuitivamente, parece que os modelos temporais baseados na abordagem de tempo discreto podem levar a uma complexidade menor por possuir um número de estados muito menor. Entretanto, esta conclusão não é de todo correta porque, na prática, com a aparição de métodos de representação do espaço de estados que usam combinações booleanas de restrições lineares de variáveis de estado, a relação de complexidade entre a abordagem de tempo denso e o número de estados deixa de ser tão direta. Uma idéia do impacto que isto pode ter na complexidade dos algoritmos de verificação pode ser vista na seguinte constatação: o problema de determinar se restrições lineares são vazias - que é um problema importante dentro dos algoritmos de verificação - é PTIME na abordagem de tempo denso e NP-completo na abordagem de tempo discreto (WANG, 2004).

Seja qual for a abordagem escolhida, os sistemas precisam ser devidamente modelados e especificados formalmente. Passaremos então a analisar este processo de especificação, notadamente com respeito à representação formal utilizada. 


\subsection{Linguagens para especificação de sistemas}

Como foi levantado na seção 2.1, o formalismo utilizado para a especificação de sistemas depende em parte da abordagem temporal escolhida. No caso da verificação de modelos a especificação é feita usando fórmulas lógicas.

Apesar da existência de diferentes linguagens ou métodos de especificação ${ }^{3}$, o objetivo do presente trabalho é estudar os modelos usados na especificação de sistemas concorrentes, entre os quais podemos citar CSP (HOARE, 1985), CCS (MILNER, 1980), Statecharts (HAREL, 1987), e as lógicas temporais (PNUELI, 1977). As lógicas temporais têm sido as mais usadas na especificação de sistemas temporizados ao lado de uma variante temporal do CSP denominada TCSP (REED; ROSCOE, 1988).

No escopo das lógicas temporais temos as seguintes semânticas:

tempo linear: é visto como uma seqüencia de eventos com um futuro determinado univocamente.

tempo ramificado: é visto como uma árvore com muitos futuros possíveis.

Pnueli (1977) propôs uma lógica com semântica de tempo linear para especificar o comportamento de programas concorrentes com dependência temporal. Esta lógica foi denominada LPTL (Linear time Propositional Temporal Logic). LPTL é uma subclasse da lógica modal que possui os operadores $\square$ que significa "começando agora em todos os estados" e $\diamond$ que significa "começando agora, existe pelo menos um estado". Também possui os operadores $U$ (until) e o (next). $p U q$ significa que " $p$ é verdade até que $q$ seja verdade" e op significa que " $p$ será verdade no próximo estado". LPTL permite a especificação só de requisitos temporais "qualitativos", isto é, não inclui referências ao tempo de forma explícita portanto só pode se pronunciar sobre a ordem parcial dos eventos, se determinados eventos ocorrem eventualmente ou não etc.

A partir do trabalho de Pnuelli, várias outras lógicas temporais foram propostas baseadas na LPTL, várias delas adaptadas para permitir a especificação de requisitos temporais "quantitativos", que incluem referências temporais e são interpretados sobre seqüencias de estados temporizados ${ }^{4}$ associando um lapso de tempo a cada estado do sistema. Tudo isto foi feito com o objetivo de especificar

\footnotetext{
${ }^{3}$ Por exemplo, alguns métodos são direcionados para a especificação de comportamento de sistemas seqüenciais como $\mathbf{Z}$ e VDM

${ }^{4}$ Para uma discussão detalhada sobre a teoria das seqüencias de estados temporizados veja (ALUR; HENZINGER, 1990).
} 
e verificar sistemas de tempo real. Exemplos destas lógicas temporais são RTL (Restricted Temporal Logic) (JAHANIAN; MOK, 1986), TPTL (Timed Propositional Temporal Logic), MTL (Metric Temporal Logic) (ALUR; HENZINGER, 1990), APTL (Asynchronous Propositional Temporal Logic) (WANG; MOK; EMERSON, 1992).

Uma vez que as lógicas com semântica de tempo ramificado admitem a possibilidade de muitos futuros, é fundamental a adição dos operadores $\exists$ e $\forall$ para especificar a relação entre estes diferentes futuros. O quantificador de caminhos $\exists$ significa "existe pelo menos um caminho a partir de agora" e $\forall$ significa "para todos os caminhos a partir de agora".

Um exemplo de lógica com semântica de tempo ramificado é a lógica CTL (Computation Tree Logic) (CLARKE; EMERSON; SISTLA, 1986). Em CTL, assim como nas suas extensões, os operadores modais de tempo linear ( $\square \diamond U \circ$ ) devem ser precedidos por um quantificador de caminhos $(\exists \forall)$. Portanto, a lógica CTL é mais expressiva que a lógica LPTL (WANG, 2004).

Assim como aconteceu com as lógicas que possuem semântica de tempo linear, varias extensões surgiram para permitir a especificação de requisitos temporais quantitativos. Emerson et al. (1990) propuseram a Real-time CTL RTCTL, que permitia especificar tempo nas fórmulas, no domínio de tempo discreto. Por exemplo, fórmulas do tipo $\exists p U^{\leq k} p$ permitem especificar comportamentos do tipo "existe uma seqüencia de estados na qual $p$ passa a ser verdadeiro em algum dos próximos $k$ estados a partir de agora".

A lógica com semântica de tempo ramificado mais utilizada em sistemas de tempo real é a Timed Computation Tree Logic TCTL (WANG, 2004). A lógica TCTL foi proposta por Alur, Courcoubetis e Dill (1990) e suporta operadores do tipo $\exists \square_{\sim c}, \exists \diamond_{\sim_{c}}, \exists U_{\sim_{c}}, \forall \square_{\sim_{c}}, \forall \diamond_{\sim_{c}}$ e $\forall U_{\sim_{c}}$. Aqui $c$ é um numero natural ${ }^{5} \mathrm{e}$ $\sim$ representa um dos símbolos binários $(<, \leq,=, \geq,>)$. Note que o operador "o" não é usado na TCTL. Isto se deve ao fato de que a TCTL usa domínio temporal denso e portanto não existe um "próximo estado" univocamente determinado ${ }^{6}$.

Henzinger et al. (1992) propuseram outra lógica que denominaram T $\mu$ (Timed $\mu$-calculus) para ser interpretada sobre árvores temporizadas. T $\mu$ é uma linguagem para especificação baseada no $\mu$-calculus que têm expressividade similar a

\footnotetext{
${ }^{5} \mathrm{Na}$ verdade podem ser usados números racionais uma vez que TCTL está definido no domínio temporal denso.

${ }^{6}$ As lógicas temporais CTL são definidas sobre árvores temporizadas e portanto os estados são formados pelas proposições válidas nesse estado e o tempo em que o sistema permanece nesse estado.
} 


\section{TCTL.}

O $\mu$-calculus é usado no domínio dos números reais, portanto o operador básico $\triangleright$ (próximo estado) muda seu significado para "até a próxima transição" observando que não existe um tempo fixo para o "próximo estado" acontecer.

Por exemplo, a fórmula $p \triangleright q$ significa que $p$ é verdadeira e continua verdadeira até que uma transição aconteça que torne $q$ verdadeira. O tempo que demora para acontecer a transição é irrelevante. Para realizar a contagem do tempo usam-se variáveis do tipo relógio. As fórmulas também podem conter fórmulas variáveis, limitadas pelos quantificadores.$u X$ e.$v X$ (respectivamente least-fixpoint e greatfixpoint $\left.{ }^{7}\right)$.

Adaptações de linguagens para programação concorrente, como a TCSP (Timed CSP) (REED; ROSCOE, 1988) também podem ser usadas para especificar e modelar sistemas de tempo real. A TCSP é uma extensão do CSP (Concurrent Systems Programming) (HOARE, 1985). Por definição, valor do tempo na TCSP é um número real, portanto o domínio temporal é denso. Vários elementos semânticos novos foram definidas nesta abordagem, como por exemplo: esperas (Wait $t)$, timeouts $\left(P_{1} \stackrel{t}{\triangleright} P_{2}\right)$ e interrupções $\left(P_{1} \stackrel{\downarrow}{t} P_{2}\right)$.

Schneider (1999) apresentou refinamentos e mecanismos de tradução de programas de CSP para TCSP, introduzindo o evento tock, que avança o tempo de uma unidade. Mostrou também como fazer a tradução inversa, isto é, como traduzir programas em TCSP para CSP.

\subsection{Formalismos para descrição de sistemas}

Em geral, os formalismos existentes para a descrição dos sistemas com tempo derivam de extensões de formalismos aplicados com sucesso para sistemas sem tempo. A seguir apresentaremos um resumo das estruturas mais usadas para modelar e verificar sistemas com tempo, destacando esta derivação, quando for apropriado.

\footnotetext{
${ }^{7} \mathrm{Na}$ teoria de ordem, um ramo da matemática, o least-fixpoint (lfp ou LFP) de uma função é o ponto fixo que é inferior ou igual a todos os outros pontos fixos, de acordo com alguma ordem parcial. Correspondentemente o great-fixpoint (gfp ou GFP) de uma função é o ponto fixo que é superior ou igual a todos os outros pontos fixos, de acordo com alguma ordem parcial.
} 


\subsubsection{Autômatos híbridos}

Os autômatos híbridos (ALUR et al., 1992) são usados para modelar sistemas embarcados. Estes autômatos possuem relógios que podem ser incrementados (decrementados) de forma independente, com diferentes taxas de variação. Restrições lineares arbitrárias são aceitas, tanto as relacionadas aos nós do grafo de transição (chamadas de invariantes) quanto as relacionadas às arestas (chamadas de condições de disparo).

Em geral, os autômatos híbridos não são usados na verificação de modelos porque neste caso, o problema da verificação de modelos é indecidível. De qualquer forma, existem algumas subclasses destas estruturas para as quais o problema da verificação de modelos é decidível. Henzinger et al. (1995) mostraram algumas das condições que fazem com que o problema da verificação de modelos sobre os autômatos híbridos seja decidível ou indecidível.

O resultado destas pesquisas mostrou que é necessário sacrificar parte da "expressividade" dos autômatos híbridos para obter algoritmos que em geral sejam PSPACE.

\subsubsection{Autômatos temporizados}

Os autômatos temporizados (Timed Automata) (ALUR; DILL, 1990) são uma subclasse especial de autômatos híbridos. Nos autômatos temporizados os relógios só podem ser incrementados a uma taxa constante, ou seja, todos os relógios evoluem simultaneamente. Além disso, só desigualdades são admitidas como restrições sobre os relógios. Apesar destas limitações, os autômatos temporizados conseguem representar de forma satisfatória sistemas discretos distribuídos.

Devido às limitações dos autômatos temporizados em relação aos autômatos híbridos, existe uma grande quantidade de resultados teóricos sobre a verificação de modelos usando autômatos temporizados (WANG, 2004). Já com respeito a complexidade, o problema da verificação de modelos sobre os autômatos temporizados é PSPACE.

Na prática, os sistemas de tempo real são usualmente modelados por um conjunto de autômatos temporizados, cada um deles representando o comportamento de um processo autônomo dentro do sistema. Este método de modelagem, facilita o processo de construção de modelos de sistemas com comportamentos concorrentes e complexos (WANG, 2004). 
Usando essa abordagem para a modelagem dos sistemas, um autômato temporizado pode ser construído como o produto de cada um desses autômatos temporizados que modelam os processos. Para poder realizar este processo, o conceito de canal para a sincronização binária de processos foi "importado" do CSP. Este conceito é conhecido na literatura como autômatos temporizados com comunicação (communicating timed automaton) ou redes de autômatos temporizados e foi introduzido por Shaw (1992). Na figura 2.1 uma rede de autômatos que modela o problema de passagem de nível é mostrada. Na figura 2.1 o autômato
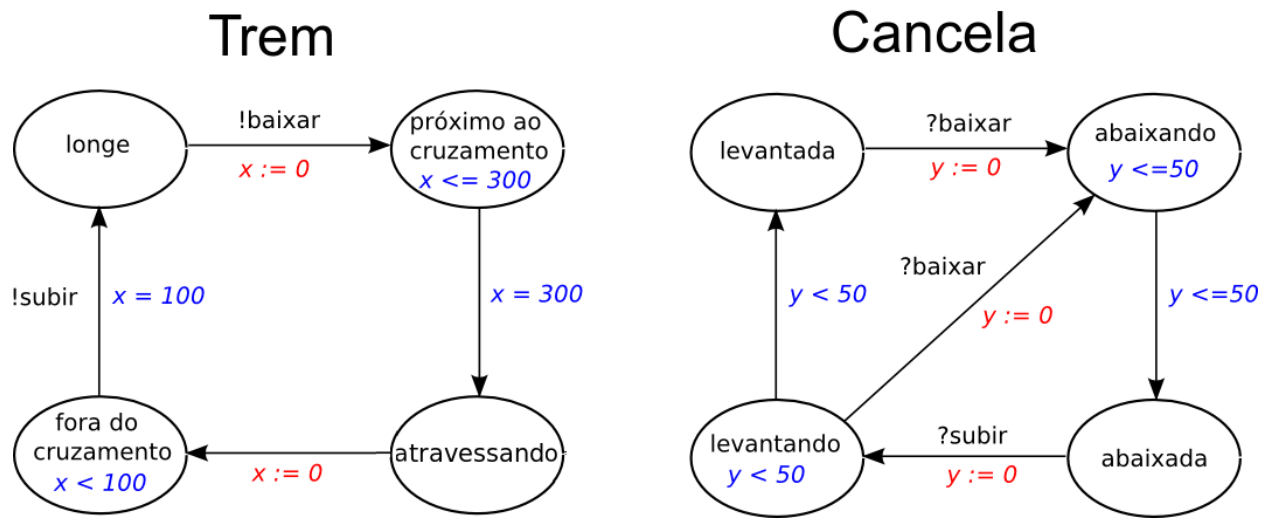

Figura 2.1: Exemplo de rede de autômatos temporizados para modelar o problema de passagem de nível.

que modela o comportamento do trem, utiliza o sinal !baixar (!subir) para sincronizar as transições de estados na rede de autômatos, o qual é representado pela transição respectiva ?baixar (?subir). Desta forma, o autômato que modela o comportamento do trem "comunica" ao autômato que modela o comportamento da cancela o momento exato de abaixar (subir), dependendo da distância entre o trem e a cancela a qual é modelada pela variável $x$. O autômato que modela o comportamento da cancela utiliza a variável y para modelar o tempo que demora as operações de abaixar e subir a cancela.

Atualmente as redes de autômatos temporizados são usadas em vários ambientes para verificação de modelos como UPPAAL, SGM, CMC e RED.

\subsubsection{Redes de Petri}

As redes de Petri têm sido usadas com sucesso na modelagem e análise de sistemas discretos (sem tempo). Similarmente ao que aconteceu na evolução de outros formalismos bem sucedidos na modelagem de problemas não temporizados, as redes de Petri também evoluíram para lidar com sistemas temporizados. Exemplos de tais extensões podem ser encontradas em (RAMCHANDANI, 1974), (SIFAKIS, 
1980) e (ZUBEREK, 1980) onde o tempo é introduzido no formalismo através da representação de lapsos ou intervalos fixos, permitindo modelar atrasos e analisar a performance dos sistemas modelados.

Outras extensões permitem modelar, além de atrasos, prazos (deadlines) o que permitiu a utilização de tais formalismos na modelagem de sistemas de tempo real. Entre estas extensões podemos citar (MERLIN; FABER, 1976), (GHEZZI et al., 1991), (TSAI; YANG; CHANG, 1995), (AALST, 1993), (CORTES; ELES; PENG, 2003). Um resumo sobre o uso das redes de Petri e suas extensões na modelagem de sistemas pode ser encontrado em (CERONE; MAGGIOLO-SCHETTINI, 1999).

As redes de Petri Temporizadas de Merlin e Faber (1976) (Time Petri Nets) possuem a maior quantidade de resultados na modelagem, análise e verificação de sistemas de tempo real (BERTHOMIEU; DIAZ, 1991), (BERTHOMIEU; RIBET; VERNADAT, 2004), (GARDEY et al., 2005).

Trabalhos recentes desenvolvidos no D-Lab mostram resultados na aplicação das RPT na verificação de sistemas de tempo real e em workflow com restrições de tempo (FOYO; SILVA, 2007), (FOYO; SILVA, 2008).

\subsubsection{Linguagens gráficas}

Entre as linguagens gráficas usadas para descrever comportamento de sistemas podemos encontrar os statecharts propostos por Harel (1987). Este formalismo permite representar sistemas complexos e concorrentes sem tempo e inspirou vários esquemas composicionais para análise e verificação de sistemas (WANG, 2004).

Modelcharts (JAHANIAN; MOK, 1994) é uma extensão temporal dos statecharts cuja semântica é definida em RTL. Neste formalismo, intervalos de tempo e eventos discretos podem ser usados conjuntamente como condições de disparo para as transições.

A UML-RT (DAMM; JOSKO; HUNGAR, 1998) é a extensão para tempo real da UML usada para especificar os modelos, que por sua vez estende a representação dinâmica de statecharts para modelcharts. Algumas ferramentas de verificação admitem este formalismo gráfico como formato de entrada porque possuem mecanismos de conversão internos implementados ou conseguem fazer a conversão usando algum módulo desenvolvido especialmente para isso. Tal é o caso da ferramenta UPPAAL que utiliza o módulo HUPPAAL para realizar esta conversão. 


\subsection{Construção do espaço de estados}

Para explorar o espaço de estados de um determinado modelo é preciso construílo. Precisamente, é na construção do espaço de estados onde reside a complexidade das abordagens de verificação de modelos.

Alur, Courcoubetis e Dill (1993) mostraram pela primeira vez que a verificação de modelos descritos através de autômatos temporizados e usando uma lógica temporal como linguagem para a especificação do sistema era decidível. Tal resultado foi obtido através da construção de um grafo com número de estados finito o qual denominaram region graph. Este método consistia em determinar o conjunto real de estados de onde evoluem as mesmas regiões ${ }^{8}$.

Este tipo de abordagem onde o espaço de estados é computado integralmente é conhecido como abordagem enumerativa. O nome dado provém do fato de que são "enumerados" todos os comportamentos possíveis para logo determinar se um dado comportamento satisfaz as especificações.

Porém, na prática, estes algoritmos mostraram-se computacionalmente irrealizáveis devido ao tamanho do grafo das regiões. Como foi mostrado em (ALUR; COURCOUBETIS; DILL, 1993) o grafo das regiões cresce exponencialmente com o número de relógios do modelo e com o maior valor de tempo usado como restrição na fórmula que está sendo verificada, enquanto cresce linearmente com a quantidade de elementos (nós + arestas) do modelo.

Devido a esta limitação, outras técnicas para a construção do espaço de estados foram introduzidas. Uma delas é conhecida como abordagem simbólica. O termo "simbólica" é usada para referir-se ao fato de que o espaço de estados não é construído nem explorado integralmente, diferenciando-se assim da abordagem enumerativa.

O problema é que para aplicar esta técnica é preciso que no modelo do sistema esteja definida uma relação específica "próximo estado" que permita computar as propriedades desejadas através da sua iteração (HENZINGER et al., 1992).

Geralmente os sistemas que usam esta abordagem são descritos usando $\mu$ calculus. A possibilidade teórica de usar a verificação de modelos simbólica através do cálculo dos fixpoints foi levantada primeiramente por Emerson e Clarke (1980) e Sifakis (1982). Porém, a técnica não se mostrou prática até que uma

\footnotetext{
${ }^{8}$ As regiões representam conjuntos de estados que evoluíram através da execução de seqüencias de transições similares e encontram-se no mesmo estado num determinado intervalo de tempo
} 
estrutura apropriada para representação dos conjuntos de estados fosse desenvolvida em (BRYANT, 1986) chamada BDD.

A abordagem simbólica foi usada com sucesso na verificação de modelos em (BURCH et al., 1990) para sistemas sem tempo. Em seguida esta abordagem foi usada em sistemas com tempo, no domínio de tempo discreto (EMERSON et al., 1990), (EMERSON; JUTLA; SISTLA, 1993). Porém, após a constatação de que para sistemas assíncronos o tempo não deve ser discretizado, a abordagem simbólica foi aplicada também ao domínio temporal denso em (HENZINGER et al., 1992).

Os algoritmos simbólicos para verificação de modelos particionam o espaço de estados em classes de equivalência de tal forma que os estados pertencentes a cada classe são equivalentes com relação ao autômato investigado e à propriedade que está sendo verificada. No pior caso, o algoritmo constrói o equivalente ao grafo de regiões de forma integral só que na prática, a complexidade do algoritmo não depende do maior valor de tempo usado como restrição na fórmula sendo verificada, como no caso da abordagem do grafo de regiões (LARSEN; PETTERSSON; YI, 1995).

Outra técnica usada para diminuir a complexidade dos algoritmos de verificação de modelos é denominada On-the-fly (HENZINGER; KUPFERMAN; VARDI, 1996), (SCHWOON; ESPARZA, 2005) (HADJIDJ; BOUCHENEB, 2006). Estes algoritmos constroem o espaço de estados do sistema investigado enquanto verificam a propriedade de interesse, de forma simultânea. O intuito é gerar apenas a parte do espaço de estados necessária para responder se a propriedade sendo verificada é satisfeita pelo modelo (WANG, 2004).

A abordagem composicional na verificação de modelos explora o fato dos sistemas serem formados por componentes. A idéia básica nesta técnica é reduzir o problema de verificação do sistema todo à verificação de seus componentes. Várias estratégias para aplicar esta idéia têm sido propostas. Laroussinie e Larsen (1998) propuseram um procedimento para refinar as fórmulas de forma iterativa reduzindo o problema de verificação, retirando basicamente os componentes que não intervém na fórmula que está sendo verificada. Neste trabalho a abordagem composicional é baseada no método de construção do grafo das regiões enquanto em (LARSEN; PETTERSSON; YI, 1995) o método de construção usado é o simbólico.

Todos os trabalhos a que fizemos referência até agora nesta seção utilizam os autômatos temporizados como formalismo para descrever o comportamento do sistema. Porém, as redes de Petri temporizadas também têm sido muito usadas nas diferentes etapas do processo de verificação de modelos. 
A introdução do conceito de classes de estados (BERTHOMIEU; MENASCHE, 1983), assim como a publicação de algoritmos para calculá-las a partir de modelos usando $\operatorname{RPTs}^{9}$ deu um impulso às pesquisas de métodos de verificação baseados em redes. A utilização desta abordagem permitiu a representação "finita" do comportamento para uma grande parte das RPTs. Este resultado, conjuntamente com os apresentados em (BERTHOMIEU; DIAZ, 1991), (YONEDA; RYUBA, 1998), levaram ao surgimento de ferramentas tais como TINA (BERTHOMIEU; RIBET; VERNADAT, 2004) e Romeo (GARDEY et al., 2005), ambas baseadas nas redes de Petri temporizadas de Merlin (MERLIN; FABER, 1976).

Apesar da abordagem das classes de estados para construir o espaço de estados ser uma abordagem enumerativa, os resultados da verificação usando este método parecem levar a complexidades menores que a reportada usando o grafo das regiões.

A redução da ordem parcial é uma técnica que pode ser usada aliada a outras aqui apresentadas. Esta técnica é baseada no fato de que raramente a ordem exata da ocorrência de certos eventos é relevante para considerar um comportamento correto, o que é garantido pelos intervalos de tempo em que os eventos especificados aconteçem.

A redução da ordem parcial tem sido usada com sucesso na verificação de sistemas sem tempo e foi estendida também para sistemas com tempo usando como formalismo base tanto os autômatos temporizados quanto as RPTs (WANG, 2004). Por exemplo, Pagani (1996) apresentou uma noção de independência entre transições baseada na semântica de tempo global dos autômatos temporizados. Yoneda e Schlingloff (1997) propuseram uma técnica de redução de ordem parcial para a verificação de modelos usando TLTL e RPTs.

\subsection{Estruturas para representação do espaço de estados}

Um dos pontos cruciais na pesquisa sobre verificação formal de sistemas são as estruturas de dados usadas para representar o espaço de estados. Para se ter uma idéia da importância destas estruturas podemos mencionar o fato de que mesmo com a descoberta dos métodos automáticos de verificação de modelos, estes modelos não puderam ser de fato aplicados a sistemas reais até a descoberta

\footnotetext{
${ }^{9} \mathrm{O}$ termo "redes de Petri temporizadas" é usado neste trabalho como referência às Time Petri Nets de Merlin; embora o termo possa ser usado também para denominar as Timed Petri Nets de Ramchandani, no presente trabalho não se faz este uso.
} 
dos grafos binários de decisão (BDD, pela suas siglas em inglês) (HENZINGER et al., 1992).

Um BDD, como proposto por (BRYANT, 1986) é uma forma mínima canônica usada na lógica proposicional para a representação simbólica de conjuntos de estados. Do ponto de vista topológico, os BDD são grafos direcionados e acíclicos com uma fonte e dois sumidouros, um para FALSE e outro para TRUE. O grafo pode representar tanto disjunções quanto conjunções sendo que cada nó é rotulado com um átomo, e os arcos emergentes com os valores do respectivo átomo. A figura 2.2 mostra alguns exemplos de BDD.
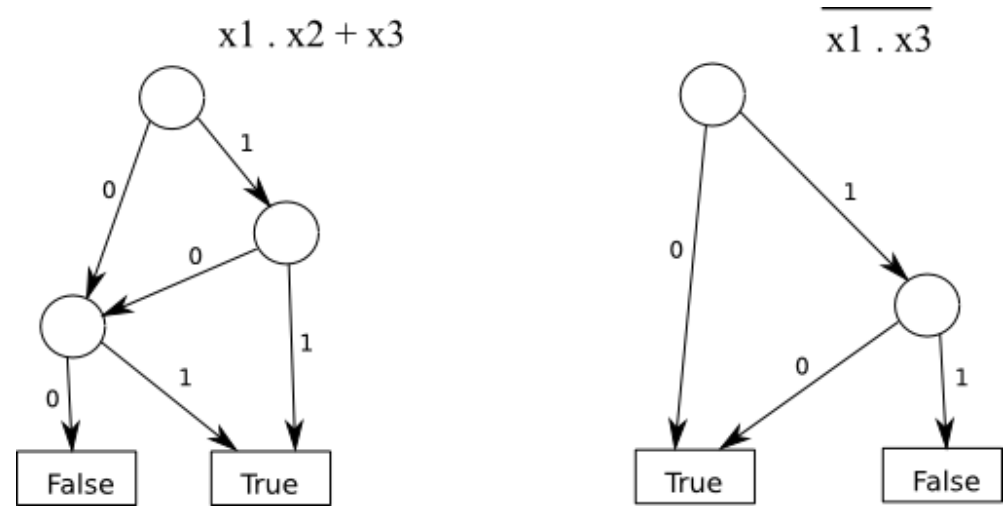

Figura 2.2: Exemplos de árvores binárias de decisão.

As árvores binárias de decisão da figura 2.2 representam duas funções lógicas. Os pesos nos arcos representam os possíveis valores lógicos da variável representada pelo nó onde os arcos são originados. Estes pesos podem tomar os valores lógicos 1 ou 0.

São as características desta estrutura que fazem dela um modo eficiente para representar e manipular os conjuntos de estados que representam. Esta estrutura é minimal porque possui o menor tamanho de representação de um espaço de estados com relação a uma ordem dada das variáveis. A estrutura é canônica porque existe somente uma representação possível para qualquer espaço de estados. Esta última característica implica que verificações da igualdade entre espaços de estados e a verificação de espaço de estados vazio podem ser feitas com elevada eficiência.

Nos sistemas temporizados foram as Matrizes de Diferenças Finitas (DBM) propostas em (DILL, 1989) que revolucionaram os verificadores de modelos. As DBM permitem representar espaços temporais nos sistemas de tempo real e são a estrutura de dados mais usada para este fim.

Uma DBM é um arranjo bidimensional na qual cada entrada registra a dife- 
rença entre dois relógios pertencentes ao espaço temporal representado pela DBM. Estas estruturas podem representar espaços de estados convexos no intervalo de tempo.

Entre as operações que podem ser realizadas com as DBMs estão a intersecção e a normalização. Esta última é muito importante porque leva à forma canônica de representação das DBMs com as vantagens que isto implica.

Os NDD (Numerical Decision Diagrams) (ASARIN et al., 1997) são baseados na idéia de que cada relógio do sistema pode tomar um valor entre 0 e $k$. A discretização do tempo leva os relógios a adotar valores $K=\{0, \ldots, k-1\}$ o que permite codificar os relógios em binários usando $\log k$ bits. Desta forma, qualquer subconjunto $K^{n}$ pode ser visto como um subconjunto de $\{0,1\}^{n \log k}$ e portanto pode ser representado por um BDD usando $n \log k$ variáveis booleanas.

Outras estruturas similares a esta têm sido propostas baseadas nas DBM e BDD. Entre elas podemos citar as seguintes: DDD (Difference Decision Diagram) (J.MøLLER et al., 1999), CDD (Clock-Difference Diagram) (BEHRMANn et al., 1999) e CRD (Clock Restriction Diagram) (WANG, 2001b).

\subsection{Ambientes Computacionais para Verificação}

Os avanços na parte teórica da verificação de sistemas de tempo real levaram ao surgimento de várias ferramentas computacionais de apoio, sendo que algumas delas atingiram o nível de produto comercial. Algumas destas ferramentas são: UPPAAL (LARSEN; PETTERSSON; YI, ), Kronos (DAWS et al., 1995), Epsilon (CERAnS; GODSKESEN; LARSEN, 1993), Romeo (GARDEY et al., 2005), HyTech (HENZINGER; HO; WONG-TOI, 1997), RED (WANG, 2001a), CMC (LAROUSSINIE; LARSEN, 1998) entre outras.

A seguir apresentaremos as principais características de algumas destas ferramentas.

UPPAAL: é um ambiente integrado para modelagem e verificação de sistemas de tempo real, modelados através de redes de autômatos, que aceitam estruturas de dados de alto nível. A ferramenta foi desenvolvida em colaboração entre as Universidades de Uppsala, na Suécia, e Aalborg na Dinamarca. Esta ferramenta usa as abordagens simbólica e composicional na construção do espaço de estados e resolve o problema de verificação de forma local. A estrutura de dados usada para representar o espaço temporal do conjunto 
de estados é a DBM. A linguagem de especificação utilizada é uma lógica modal temporizada denominada $\mathscr{L}_{s}$ que pode ser vista como um fragmento da lógica $\mathrm{T} \mu$, a qual pode ser convertida a $\mathrm{TCTL}^{10}$. Tem ainda a capacidade de gerar contraexemplos.

Kronos: é um verificador de sistemas modelados usando autômatos temporizados e foi desenvolvido no VERIMAG, França, um renomado centro de pesquisas em sistemas embarcados (www-verimag.imag.fr). Usa algoritmos de atingibilidade backward e forward para verificar as propriedades especificadas através da TCTL. A ferramenta constrói o espaço de estados usando as abordagens simbólica e on-the-fly e resolve o problema de verificação de forma local. As estruturas de dados usadas são DBMs e NDDs. Tem também a capacidade de gerar contraexemplos.

Romeo: é um ambiente para modelagem, análise e verificação de sistemas usando RPTs ou S-TPNs. A ferramenta foi desenvolvida no IRCCyN, Nantes, França. Além do editor gráfico para as RPT, o pacote possui dois módulos: i) o GPN é o verificador de modelos que consegue verificar propriedades de segurança definidas sobre as RPT usando a abordagem enumerativa baseada nas classes de estado para a construção do espaço de estados; ii) o Mercutio converte o modelo em RPT num autômato temporizado que pode ser verificado usando alguma outra ferramenta (UPPAAL, Kronos, HyTech, etc.).

HyTech: é um verificador de modelos para sistemas híbridos lineares. A especificação das propriedades é feita usando ICTL e o sistema é modelado usando autômatos híbridos. A ferramenta foi desenvolvida na Universidade de Berkeley, Califórnia, Estados Unidos. Gera contraexemplos e também pode realizar a análise de parâmetros do modelo ainda que a terminação desta análise não seja garantida.

CMC: é um verificador de modelos que utiliza redes de autômatos para a descrição dos sistemas e verifica as propriedades especificadas usando a lógica modal temporizada $\mathscr{L}_{s}$. A ferramenta foi desenvolvida no Laboratório de Especificação e Verificação de Cachan na França em colaboração com a Universidade de Aalborg, Dinamarca. Usa a abordagem composicional, reduzindo gradativamente os componentes do sistema e incorporando-os na fórmula. A eficiência do método depende do uso do subconjunto da lógica

\footnotetext{
${ }^{10} \mathrm{Na}$ verdade esta linguagem só consegue representar um subconjunto da TCTL
} 
$\mathscr{L}_{s}$ que pode ser calculado usando só cálculos que envolvem least-fixpoints ${ }^{11}$.

\subsection{Trabalhos relacionados}

A proposta desta tese é usar uma extensão das redes de Petri para modelar sistemas distribuídos que permita a verificação do modelo de forma eficiente. Como nosso foco são sistemas distribuídos, que envolvem os sistemas de tempo real, faremos referência a abordagens similares, ou seja, com capacidade para representar incertezas na duração dos processos.

Entre as extensões das redes de Petri com esta capacidade podemos citar as RPT (MERLIN; FABER, 1976), ITCPN (AALST, 1993), TCPN (TSAI; YANG; ChAnG, 1995), S-TPNs (ROUX; DéPlAnche, 2002), PRES+ (CORTES; ElES; PENG, 2003). Na maioria delas o problema da verificação de modelos foi abordado de diferentes maneiras, e por isso vamos resumir aqui as similaridades e diferenças destas abordagens com a nossa proposta.

Em (LIME; ROUX, 2006), as classes de estado geradas a partir do modelo em RPT são usadas para construir um tipo especial de autômato temporizado denominado autômato de classes de estado o qual possui um comportamento bisimilar em tempo ao do modelo em RPT. Este autômato gerado possui um número reduzido de variáveis relógios ${ }^{12}$ o que pode ser verificado usando uma das ferramentas disponíveis para verificação de modelos com autômatos temporizados. Também estenderam a TCTL para expressar propriedades sobre as marcações das redes de Petri.

Enquanto esta abordagem usa as classes de estado lineares para construir o autômato a nossa proposta é usar as classes de estado atômicas, as quais preservam as propriedades da lógica de tempo ramificado (YONEDA; RYUBA, 1998). Outra diferença é que a verificação de propriedades quantitativas é feita usando a abordagem dos "observadores" a qual transforma o problema da verificação temporal em problemas de atingibilidade (TOUSSAINT; SIMONOT-LION; THOMESSE, 1997), enquanto na nossa proposta o processo de verificação do modelo é feito sobre uma espécie de grafo de atingibilidade construído usando as classes de estado atômicas sem precisar mudar de formalismo de representação e permitindo a verificação da lógica TCTL de forma integral ${ }^{13}$.

\footnotetext{
${ }^{11}$ Foi provado que cálculos de propriedades que envolvem iterações de least-fixpoints e greatfixpoints possuem complexidade exponencial no número de mudanças do tipo de aproximação

${ }^{12}$ Os autores alegam que conseguem o ótimo ou algo perto do ótimo.

${ }^{13}$ quando UPPAAL é usado como ferramenta de verificação só é possível verificar um subconjunto desta lógica
} 
Uma abordagem similar é usada em (CORTES; ELES; PENG, 2003), onde o sistema é modelado usando uma extensão das redes de Petri denominadas PRES+ e transformada em autômato temporizado para ser verificado usando uma das ferramentas disponíveis.

Aalst (1993) propôs uma extensão das redes de Petri coloridas (JENSEN, 1992) para modelar o comportamento temporal de sistemas de tempo real similar ao da nossa proposta de extensão temporal para as redes GHENeSys (General Hierarchical Enhanced Net System). No mesmo trabalho foi proposto um método para a construção do grafo de atingibilidade de forma reduzida. Este método foi complementado e melhorado em (BERTHELOT, 1994) para construir as classes de estado. Diferentemente da nossa proposta, os intervalos das classes de estado são colocados num relógio global enquanto nós colocamos estes intervalos como incrementos no relógio global o que facilita a modelagem com um número finitos de estados. Apesar das melhorias introduzidas em (BERTHELOT, 1994), nenhuma estrutura canônica foi proposta para a determinação de equivalência entre classes o que leva a algoritmos pouco eficientes.

Christensen, Kristensen e Mailund (2001) propuseram um método para gerar as classes de estados para uma extensão temporizada das redes Lugar-Transição. Nesta abordagem o domínio temporal é discreto. No mesmo trabalho foi mostrada a possibilidade de verificação da lógica RTCTL sobre uma estrutura gerada a partir do citado método.

Em (VIRBITSKAITE; POKOZY, 1999) foi proposto um método para verificar propriedades descritas usando a lógica TCTL sobre RPT's seguras. Neste trabalho, a estrutura sobre a qual as propriedades serão verificadas é gerada usando a abordagem do grafo das regiões e uma técnica de ordem parcial para reduzir o espaço de estados e portanto a eficiência do verificador. O fato de aceitar só RPT's seguras reduz a expressividade do formalismo usado na modelagem de sistemas. Por outro lado, o uso do grafo das regiões na construção do espaço de estados limita a eficiência do método devido a sua complexidade.

Wang, Deng e Xu (2000) também usaram a abordagem das classes de estados (as quais denominaram de clock-stamped state class) na construção da árvore de atingibilidade para RPT's onde colocam de forma explícita o intervalo de tempo transcorrido em cada uma das transições. Como o método de verificação é baseado na árvore de atingibilidade o mesmo não é utilizável para sistemas cíclicos, os quais, mesmo sendo representados por RPT limitadas geram uma árvore de atingibilidade infinita. Esta abordagem é baseada nas classes de estados 
lineares e portanto só permite a verificação de propriedades temporais lineares. Pelo tamanho da estrutura onde as verificações podem ser feitas podemos concluir que este método dificilmente seria aplicavél em situações reais.

Lakos e Petrucci (2007) mostraram o efeito da aplicação de uma abordagem modular (o que seria um equivalente a abordagem composicional usada nos autômatos temporizados) na verificação de propriedades dos sistemas distribuídos com nível baixo de acoplamento entre os seus módulos.

Os trabalhos de Berthomieu e colaboradores, (BERTHOMIEU; MENASCHE, 1983); (BERTHOMIEU; DIAZ, 1991); (BERTHOMIEU; RIBET; VERNADAT, 2004); e (BERTHOMIEU; VERNADAT, 2003) assim como os de Yoneda e Ryuba (1998); Boucheneb e colaboradores (BOUCHENEB; ALGER; BERTHELOT, 1993); (BOUCHENEB; HADJIDJ, 2006); (BOUCHENEB; RAKKAY, 2007) e Penczek e Półrola (2001) sobre a geração das classes de estado a partir das RPT constituem uma base fundamental para o desenvolvimento deste trabalho. Entretanto, diferentemente destes trabalhos, nós abordamos a construção de estruturas onde as propriedades quantitativas podem ser verificadas de forma direta através das fórmulas em TCTL. Além disso, desenvolvemos os algoritmos de verificação para TCTL para a resolução do problema de verificação de modelos global e local.

Recentemente Hadjidj e Boucheneb (2008) abordaram o problema da verificação de um subconjunto da TCTL para sistemas modelados usando as RPT, usando técnicas on-the-fly. Nesta abordagem, uma RPT especial em paralelo com a RPT que modela o sistema a ser analisado, é usada para verificar a propriedade com tempo. Os resultados obtidos usando a abordagem aqui proposta serão comparados com os resultados usando a abordagem supra-citada.

\subsection{Resumo e comentários finais}

Pelo que foi apresentado neste capítulo, seria possível concluir que os verificadores de modelos baseados em redes de Petri não conseguem melhorar a eficiência daqueles baseados em autômatos temporizados. Esta conclusão estaria sustentada nos seguintes fatos:

- Os sistemas baseados na abordagem enumerativa são ineficientes porque precisam construir o espaço de estados inteiro antes de proceder à verificação de uma fórmula.

- As abordagens conhecidas para construir o espaço de estados de um sistema 
modelado através de extensões temporizadas das redes de Petri, apropriadas para a modelagem de sistemas de tempo real, são enumerativas (grafo das regiões ou classes de estados).

- Não é possível aplicar abordagens simbólicas se não for possível definir adequadamente as relações de "próximo estado" o que por enquanto não é possível aplicar no caso das redes de Petri com tempo a não ser transformandoas em autômatos temporizados com comportamentos equivalentes, o que a nosso modo de ver carece de sentido ${ }^{14}$.

- O uso da técnica on-the-fly é limitado pelo fato de que o espaço de estados sempre é construído usando métodos forward partindo do estado inicial, de modo que, mesmo que não seja preciso construir o espaço de estados completamente, uma parte do espaço construído carece de utilidade para determinar se a fórmula é válida ou não.

Então por que insistir em abordagens de verificação usando extensões das redes de Petri? Em (GIRAULT; VALK, 2001); (DAVID; ALLA, 2005) podemos encontrar várias razões para defender o uso destas extensões, mas nenhuma delas nos leva a intuir que estas poderiam levar a melhores resultados em complexidade que os autômatos temporizados como sugerem os fatos previamente mencionados. Sendo assim, vamo-nos concentrar nos seguintes fatos:

- Mesmo usando o algoritmo mais eficiente para verificar modelos usando autômatos temporizados, a complexidade continua exponencial no tamanho do modelo e no número de variáveis relógio do modelo.

- A redução no tamanho do modelo, aplicando abordagens on-the-fly e composicional, é conseguida ao custo de construir o espaço de estados para cada fórmula a ser verificada.

- O processo de verificação de propriedades sobre a estrutura que representa o espaço de estados do sistema uma vez construído é polinomial mesmo para lógicas de tempo ramificado e usando domínio temporal denso.

Existe um outro fato interessante neste processo como um todo. Se um sistema for especificado de forma correta, devem existir fórmulas, temporais ou não, para cada uma das funcionalidades desejadas. Por outro lado, no modelo

\footnotetext{
${ }^{14}$ Se a verificação vai acontecer de fato sobre um autômato temporizado porque não modelar o sistema desde o começo como autômato, eliminando o processo de transformação, que tem certa complexidade, além da possibilidade de inclusão de erros durante o processo.
} 
do sistema não devem figurar módulos ou componentes que não contribuem com funcionalidades para o sistema como um todo. Isto indica que, mesmo que o espaço de estados do sistema seja construído por partes, existe a possibilidade (a qual não é pequena no nosso entendimento) de na soma, construir-se um espaço de estados maior que o espaço de estados completo construído de uma vez só.

Valendo-se dessa hipótese, podemos pensar que existe espaço para propostas que usam abordagens enumerativas para construir o espaço de estados completo (o que de fato é feito em tempo e espaço exponencial) e logo verificar cada uma das propriedades em tempo polinomial sem precisar construir novamente o espaço de estados o que no final pode nos levar a uma redução na complexidade do processo de verificação.

Levando em consideração estes argumentos, temos ainda o problema de obter algoritmos de construção do espaço de estados cuja complexidade não seja função do valor das constantes de tempo das fórmulas verificadas. Um passo como este, colocaria as abordagens baseadas em extensões das redes de Petri, no pior caso, em igualdade de condições com os métodos de verificação baseados em autômatos temporizados que usam abordagem simbólica.

Como veremos a seguir, isto já foi tentado, aplicando a abordagem das classes de estados nas redes de Petri temporizadas como mostram os trabalhos de Berthomieu e Vernadat (2003); Yoneda e Ryuba (1998); Boucheneb, Alger e Berthelot (1993) e Hadjidj e Boucheneb (2008). 


\section{$3 \quad$ Uma Abordagem Baseada em Redes de Petri para a Verificação de Propriedades Quantitativas}

Como foi mostrado no capítulo anterior, os métodos enumerativos são a opção quando extensões das redes de Petri são usadas como formalismo para descrever o comportamento do sistema. Um resultado de sucesso nesta abordagem seria conseguir uma complexidade similar à obtida usando os métodos simbólicos sem ter de sacrificar expressividade da especificação.

Desde o começo deste trabalho, deixou-se clara a intenção de incorporar à rede GHENeSys um mecanismo de verificação e ao mesmo tempo aumentar sua expressividade para modelar sistemas temporizados. Dado o tipo de sistema que pretende-se abarcar neste trabalho, algumas escolhas foram feitas para o esquema de verificação: i) como foi levantado na pesquisa bibliográfica, o modelo temporal adequado para sistemas distribuídos é o de tempo denso; ii) também pode-se constatar que a lógica temporal mais usada para este tipo de sistemas é a TCTL.

Como foi explicado no capítulo anterior, não se considerou viável usar as RPTs apenas como uma ponte entre modelagem e verificação como é proposto em (LIME; ROUX, 2006). Se for para usar as RPT apenas para sintetizar um autômato temporizado onde as verificações podem ser feitas de fato, é melhor modelar o sistema de uma vez usando autômatos temporizados. Se as RPTs são um formalismo adequado para a modelagem de sistemas de tempo real, e a abordagem das classes de estados particiona o espaço de estado de forma que as propriedades que precisam ser verificadas são conservadas, então seria melhor projetar algoritmos de verificação sobre esta partição em vez de usá-la apenas como ponto de partida para a construção de um autômato temporizado.

Uma solução como esta só faria sentido se a complexidade do processo de 
verificação sobre as RPTs fosse comparável à soma das complexidades dos processos de construção do autômato temporizado usando a abordagem das classes de estados e da verificação das propriedades no autômato construído. Na opinião do autor, a verificação do modelo diretamente nas RPTs é não só possível como viável, o que pretende-se mostrar neste capítulo.

Abordagens que usam extensões das redes de Petri com capacidade para verificação de propriedades expressas por fórmulas temporais (exceto aquelas que usam o método de transformação para autômatos temporizados) usam o método enumerativo para a construção do espaço de estado.

Em (VIRBITSKAITE; POKOZY, 1999) um esquema para verificação da TCTL é apresentado, onde o método do grafo de regiões é usado na construção do espaço de estados e uma técnica de redução da ordem parcial é usada em seguida para reduzir o tamanho deste grafo. Outro trabalho importante é o de Yoneda e Schlingloff (1997) onde um esquema para verificação de uma lógica denominada TNL $^{1}$ é apresentado, usando as classes de estado na construção do espaço de estados e, similarmente ao método anterior, uma técnica de redução da ordem parcial para reduzir o tamanho do espaço construído. Wang, Deng e Xu (2000) propuseram um método para calcular a duração de cada atividade a partir das classes de estados lineares o que permite determinar a validade de propriedades lineares quantitativas. Todas estas abordagens usam as RPT como formalismo para modelar o sistema.

Para uma melhor compreensão da proposta aqui apresentada a mesma será formulada usando como base as RPT o que facilitará na comparação dos resultados obtidos com outras abordagens. A definição temporal da rede GHENeSys será apresentada de forma análoga à definição das RPT, de forma que o esquema de verificação proposto possa ser transferido para as redes GHENeSys. Finalmente será discutido como os mecanismos de hierarquia da GHENeSys podem ajudar no tratamento dos algoritmos para o processo de verificação.

No caso dos sistemas temporizados, a verificação de modelos é uma técnica que depende de três fatores: i) da construção de um modelo finito do sistema onde as propriedades que se desejam verificar sejam preservadas; ii) da especificação das propriedades desejadas em uma lógica temporal e, iii) dos algoritmos que determinam se uma determinada fórmula é satisfeita no modelo.

A seguir tratam-se cada um destes aspectos, mas antes disso será feita uma

\footnotetext{
${ }^{1}$ Trata-se de uma lógica linear similar à LTL, adaptada para ser usada na especificação de propriedades em redes
} 
breve apresentação das RPT de Merlin.

\subsection{As redes de Petri temporizadas}

As RPT de Merlin são redes de Petri com intervalos de tempo associados às transições. Formalmente, uma RPT é uma tupla $\left(P, T\right.$, Pre, Pos, $\left.M_{0}, I s\right)$ donde $P$ e $T$ são conjuntos finitos de lugares e transições respectivamente, tal que $P \cap T=$ $\emptyset$, Pre e Pos são as funções relacionais (Pre, Pos $: P \times T \rightarrow N, M_{0}$ é a marcação inicial (uma instância específica do mapeamento $P \rightarrow N$ ) e $I s: T \rightarrow Q^{+} \times\left\{Q^{+} \cup\right.$ $\infty\}$ associa a cada transição $t \in T$ um intervalo $[E F T(t), L F T(t)]$ denominado de intervalo estático de disparo de $t$. $E F T(t)$ e $L F T(t)$ são respectivamente os prazos mínimo e máximo para o disparo de $t$. $N$ e $Q^{+}$são respectivamente o domínio dos números naturais e o domínio dos números racionais não negativos.

Seja $M$ uma marcação e $t$ uma transição. Diz-se que a transição $t$ está habilitada pela marcação $M$ se e somente se existe em $M$ o número de marcas requeridas para o disparo de $t$, formalmente: $\forall p \in P, M(p) \geq \operatorname{Pre}(p, t)$. O conjunto das transições habilitadas pela marcação $M$ é denotado como enb $(M)$, formalmente: $\operatorname{enb}(M)=\{t \in T \mid \forall p \in P, \operatorname{Pre}(p, t) \leq M(p)\}$.

Seja $M$ a marcação atingida através do disparo da transição $t_{f}$ a partir de uma dada marcação. O conjunto das transições recém habilitadas em $M$ é denotado como new $\left(M, t_{f}\right)$, formalmente: new $\left(M, t_{f}\right)=\left\{t \in \operatorname{enb}(M) \mid t=t_{f} \vee \exists p,(M(p)-\right.$ $\left.\left.\operatorname{Pos}\left(p, t_{f}\right)\right) \leq \operatorname{Pre}(p, t)\right\}$.

Existem na literatura basicamente duas definições para estados nas RPT. A primeira é baseada em relógios, que associa um relógio a cada transição habilitada pela marcação do estado. Estes relógios medem o tempo desde que a transição foi habilitada pela última vez. A segunda é baseada em intervalos, e define o estado como uma marcação e uma função que associa um intervalo de tempo fechado a cada transição habilitada. Este é o intervalo válido de disparo da transição no estado em questão.

Nesta tese será usada a segunda definição devido às vantagens sobre a primeira no cálculo de abstrações do espaço de estados as quais preservam as propriedades lineares e de atingibilidade (BERTHOMIEU; VERNADAT, 2003). Mesmo assim, sempre que fazamos referência a "estados concretos" estaremos usando a primeira definição. Note que, sendo assim um estado está composto por um número infinito de estados concretos.

Portanto, o estado em uma RPT é um par $s=(M, I)$ onde $M$ é uma marcação 
e $I$ é uma função de intervalos $I s: \operatorname{enb}(M) \rightarrow Q^{+} \times\left\{Q^{+} \cup \infty\right\}$.

Assim, um estado $s=(M, I)$ e uma transição $t \in e n b(M)$, o intervalo $I(t)$ é denominado o intervalo de disparo de $t$. Os valores mínimos e máximos do intervalo $I(t)$ são denotados por $\downarrow I(t)$ e $\uparrow I(t)$.

Uma transição $t_{f} \in T$ é disparável em um estado $s=(M, I)$ sse $t_{f} \in e n b(M)$ e $I\left(t_{f}\right)$ é consistente. Por sua vez, um intervalo de disparo é consistente sse:

$$
\forall t \in \operatorname{enb}(M) \downarrow I\left(t_{f}\right) \leq \uparrow I(t)
$$

Denomina-se $\Upsilon(M)$ ou $\Upsilon(s)$ ao conjunto das transições disparáveis em $s$.

O estado inicial de uma RPT é $s_{0}=\left(M_{0}, I_{0}\right)$ onde $I_{0}=I(t), \forall t \in e n b\left(M_{0}\right)$.

Os estados podem evoluir tanto por transições discretas como contínuas. As primeiras são resultado do disparo de transições na rede e as segundas são o resultado da passagem do tempo. Nesta tese será usada apenas a primeira delas.

A transição entre dois estados $s=(M, I)$ e $s^{\prime}=\left(M^{\prime}, I^{\prime}\right)$ através do disparo da transição $t$ em um intervalo $I(t)$ é denotado como: $s \stackrel{t, I(t)}{\longrightarrow} s^{\prime}$

O espaço de estados de uma RPT é a estrutura $\left(S, \rightarrow, s_{0}\right)$, onde $s_{0}=\left(M_{0}, I_{0}\right)$ é o estado inicial, $S=\left\{s \mid s_{0} \stackrel{*}{\rightarrow} s\right\}$, e onde $s_{0} \stackrel{*}{\rightarrow}$ é o fechamento (closure) reflexivo e transitivo da relação de transição de estados. $S$ é o conjunto de todos os estados atingíveis a partir de $s_{0}$.

Uma marcação $M$ é dita atingível se somente se $\exists s \in S$ tal que $s=(M, I)$.

\subsection{A lógica TCTL}

A lógica TCTL foi proposta em (ALUR; COURCOUBETIS; DILL, 1990) como uma extensão da CTL (CLARKE; EMERSON; SISTLA, 1986). O propósito era permitir a especificação de propriedades quantitativas, que são de vital importância no design de sistemas de tempo real. Nesta seção vamos mostrar como a lógica TCTL, que foi definida como uma linguagem para especificação de sistemas com tempo, modelados através de autômatos temporizados, pode ser estendida para ser usada em sistemas modelados em RPT.

Lime e Roux (2006) propuseram uma adaptação da TCTL para definir propriedades sobre as RPT e estenderam esta lógica para determinar algumas propriedades das redes de Petri que acharam interessantes. A seguir apresentaremos como especificar fórmulas da TCTL sobre as RPT para em seguida analisar a 
possibilidade ou necessidade de estender esta linguagem.

Em CTL podem-se escrever por exemplo fórmulas como $\exists \diamond p$, estipulando que em um determinado caminho ou processo, a proposição $p$ em algum momento será verdadeira. Na TCTL limites de tempo podem ser adicionados aos operadores temporais $\diamond, \square, U$, que respectivamente significam "em algum momento", "sempre" e "até". Estes operadores têm que ser usados em conjunto com os quantificadores de caminhos $\exists$ e $\forall$ que significam "existe" e "para todos" respectivamente.

Por exemplo, a fórmula $\exists \diamond_{\leq 5} p$ significa existe um caminho no qual a proposição $p$ é verdadeira em no máximo 5 unidades de tempo.

Seja $P$ um conjunto de proposições e $Q^{+}$o conjunto dos números racionais positivos.

Definição 1 (TCTL sintaxe). As fórmulas $\phi$ da TCTL são definidas indutivamente da seguinte forma:

$$
\phi:=p \mid \text { false }|\neg \phi| \phi_{1} \wedge \phi_{2}\left|\phi_{1} \rightarrow \phi_{2}\right| \exists\left[\phi_{1} U_{\sim_{c}} \phi_{2}\right] \mid \forall\left[\phi_{1} U_{\sim_{c}} \phi_{2}\right]
$$

onde $p \in P, c \in Q^{+}$, e $\sim$ é uma das seguintes relações binárias $<, \leq,=, \geq$, ou $>$.

Note que em TCTL, não existe o operador "próximo estado" uma vez que o domínio temporal é denso, o que impede a determinação de um único próximo estado.

Os operadores temporais também podem ser definidos usando intervalos de tempo. Por exemplo, $\exists \diamond_{(a, b)} \phi$, especifica que $\phi$ é válida pelo menos uma vez no intervalo de tempo $(a, b)$ em algum caminho. Essa fórmula equivale à fórmula $\exists \diamond_{=a} \phi \exists \diamond_{<(b-a)} \phi$.

Existem outras abreviaturas aceitas na TCTL. Algumas delas aparecem na tabela 3.1 e outras podem ser deduzidas a partir destas. Os operadores de caminhos que não possuem limites de tempo correspondem a operadores TCTL no qual o limite temporal é $\geq 0$ estabelecendo assim equivalência com os respectivos operadores em CTL. Por exemplo, $\exists \diamond \phi$ corresponde a $\exists \diamond_{\geq 0} \phi$.

A validade destas fórmulas é determinada sobre uma estrutura que especifica um conjunto de estados rotulados com as proposições válidas em cada um deles, e uma relação entre os estados que permite associar seqüências computacionais 


\begin{tabular}{|c|c|c|}
\hline Fórmula & Abreviatura & Significado \\
\hline$\forall \diamond_{\sim_{c}} f$ & $\forall\left[\right.$ true $\left.U_{\sim c} f\right]$ & $\begin{array}{l}f \text { se torna válida em algum caminho começando } \\
\text { em } s_{0} \text { em (no máximo, no mínimo, exatamente) c } \\
\text { unidades de tempo }\end{array}$ \\
\hline$\exists \diamond_{\sim c} f$ & $\exists\left[\right.$ true $\left.U_{\sim c} f\right]$ & $\begin{array}{l}\text { existe algum caminho começando em } s_{0} \text { que leva } \\
\text { a um estado em que } f \text { é válida em(no máximo, no } \\
\text { mínimo, exatamente) c unidades de tempo }\end{array}$ \\
\hline$\forall \square_{\geq 0} f$ & $\neg \exists \diamond_{\geq 0}[\neg f]$ & $\begin{array}{l}f \text { é válida em cada estado de todos os caminhos } \\
\text { que começam em um determinado estado } s\end{array}$ \\
\hline$\exists \square_{\geq 0} f$ & $\neg \forall \diamond_{\geq 0}[\neg f]$ & $\begin{array}{l}\text { existe algum caminho começando em um deter- } \\
\text { minado estado } s \text { no qual } f \text { é válida em todos os } \\
\text { estados }\end{array}$ \\
\hline
\end{tabular}

Tabela 3.1: Algumas abreviaturas de formulas TCTL

a cada um. A semântica das fórmulas TCTL é definida sobre uma estrutura que representa o comportamento temporal "ramificado" do sistema.

As fórmulas podem ser separadas em dois tipos: fórmulas para estados e para caminhos. As fórmulas para estados dependem somente das proposições válidas no estado que está sendo verificado. Já as fórmulas para caminhos são determinadas verificando os caminhos que começam no estado onde a fórmula é verificada. Obviamente fórmulas de ambos os tipos podem aparecer permitindo especificar comportamentos combinados.

Uma seqüência computacional, ou simplesmente uma seqüência em uma estrutura para verificação da TCTL é um caminho no espaço de estados $S$ composto por uma sucessão de transições disparáveis começando em qualquer estado $s \in S$. O conjunto de todos os caminhos possíveis nesta estrutura será denotado por $\lceil\lambda\rceil$.

Seja $\omega \in\lceil\lambda\rceil$ um caminho começando no estado $s_{0} \in S$.

$$
\omega=s_{0} \stackrel{a_{1}, d_{1}}{\longrightarrow} s_{1} \stackrel{a_{2}, d_{2}}{\longrightarrow} \ldots \stackrel{a_{n-1}, d_{n-1}}{\longrightarrow} s_{n-1} \stackrel{a_{n}, d_{n}}{\longrightarrow} s_{n}
$$

onde $\forall i \in[1 . . n]$, existem $l_{i}, u_{i}$ tal que $l_{i} \leq d_{i} \leq u_{i}$.

O conjunto de estados em um caminho $\omega$ é denotado como $S_{\omega}$ e o tempo necessário para percorrê-lo como $t_{s_{n}}$. Obviamente $t_{s_{n}}=\sum_{i=1}^{n} d_{i}$.

Para determinar a validade das fórmulas com operadores temporais é necessário conhecer o tempo máximo ou mínimo (dependendo da relação binária usada na restrição temporal subscrita ao operador da fórmula) das seqüencias computacionais que satisfazem a parte lógica da fórmula.

Seja $G$ uma estrutura onde é possível verificar a TCTL ${ }^{2}$. O significado de

\footnotetext{
${ }^{2}$ A definição de estrutura TCTL e como construí-la será abordada em detalhes na seção 3.3
} 
uma determinada fórmula TCTL ser verdadeira ou válida em um determinado estado em uma estrutura TCTL é definido como:

Definição 2 (Satisfatibilidade). Para uma estrutura TCTL $G$, um estado $s \in$ $S$, e uma fórmula TCTL $\phi$, a relação de satisfatibilidade é definida indutivamente como segue:

$$
\begin{aligned}
& s \models \phi \text { sse } \phi \in \mu(s), \text { onde } \mu(s) \text { é o conjunto das fórmulas atômicas } \\
& \text { verdadeiras em } s \\
& s \not \models \text { false } \\
& s \models \neg \phi \quad \text { sse } s \not \models \phi \\
& s \models\left(\phi_{1} \rightarrow \phi_{2}\right) \text { sse } s \not \models \phi_{1} \text { ou } s \models \phi_{2} \\
& s \models\left(\phi_{1} \vee \phi_{2}\right) \text { sse } s \models \phi_{1} \text { ou } s \models \phi_{2} \\
& s \models\left(\phi_{1} \wedge \phi_{2}\right) \text { sse } s \models \phi_{1} \text { e } \quad s \models \phi_{2} . \\
& s \models \exists\left[\phi_{1} U_{\sim c} \phi_{2}\right] \text { sse } \exists \omega \in\lceil\lambda\rceil, \exists t \sim c, \forall s^{\prime} \in S_{\omega} \backslash\left\{s_{n}\right\}, 0 \leq t_{s^{\prime}} \leq t s^{\prime} \models \phi_{1} \\
& \quad e t=t_{s_{n}}, s_{n} \models \phi_{2} \\
& s \models \forall\left[\phi_{1} U_{\sim_{c}} \phi_{2}\right] \text { sse } \forall \omega \in\lceil\lambda\rceil, \quad \exists t \sim c, \forall s^{\prime} \in S_{\omega} \backslash\left\{s_{n}\right\}, 0 \leq t_{s^{\prime}} \leq t s^{\prime} \models \phi_{1} \\
& \quad e t=t_{s_{n}}, s_{n} \models \phi_{2}
\end{aligned}
$$

Uma fórmula TCTL $\phi$ é satisfativel se e somente se existir uma estrutura TCTL $G$ e um estado s de $G$, tal que $(G, s) \models \phi . \quad((G, s) \models \phi$ pode ser escrito simplesmente como $s \models \phi$ uma vez que a estrutura é fixa).

Note que a sub-fórmula $\exists \diamond p U q$ é uma forma abreviada de $\exists \diamond p U_{\geq 0} q$.

O problema de satisfatibilidade para a lógica TCTL é indecidível para os autômatos temporizados (ALUR; COURCOUBETIS; DILL, 1993), mas existem subconjuntos decidíveis desta lógica (TORRE; NAPOLI, 2000).

Como o objetivo deste trabalho é a verificação de modelos o problema de satisfatibilidade não será abordado.

\subsubsection{Estendendo a TCTL}

(LIME; ROUX, 2006), além de propor uma adaptação da TCTL para as RPT denominadas TPN-TCTL (Timed Computation Tree Logic for Time Petri Nets) também tratou de incluir algumas propriedades associadas às redes de Petri: 
tipo 1: determinação do tempo decorrido entre duas marcações.

tipo 2: determinação do tempo decorrido entre duas transições.

tipo 3: determinação do tempo necessário para atingir determinada marcação.

Estas propriedades podem ser verificadas através da lógica TCTL sem a necessidade de se aumentar a sintaxe. O problema de fato é a eficiência com que esta verificação pode ser feita.

Por exemplo, suponha-se que é preciso determinar se o tempo transcorrido entre duas marcações $M_{1}=(p 1, p 2)$ e $M_{5}=(p 4)$ é menor que 8 unidades de tempo. Usando a lógica TCTL, esse problema é equivalente à verificação da fórmula $\exists \diamond\left(p 1 \wedge p 2 \wedge \exists \diamond_{\leq 8} p 4\right)$. Como a ocorrência das transições está associada às marcações, as propriedades do tipo 2 podem ser verificadas usando uma abordagem similar àquela para o tipo 1. As propriedades do tipo 3 são claramente um subconjunto das de tipo 1.

A validade das propriedades pode ser determinada usando um verificador projetado para resolver o problema da verificação de modelos que será abordado na seção seguinte. O problema da eficiência aparece precisamente pelo fato de se ter que avaliar as sub-fórmulas em todos os estados da estrutura.

Para melhorar a eficiência poderíamos sugerir um algoritmo para determinar este tipo de propriedades que, no pior caso, varreria o espaço de estado completo. Desta forma podemos aumentar a performance na verificação de tais propriedades.

Por fim, a verificação das propriedades estendidas resume-se à possibilidade de determinar o tempo transcorrido entre conjuntos de estados (ou apenas um) que satisfazem a uma determinada condição, por exemplo, que tenha uma marcação determinada. A condição para determinar esta propriedade é que nenhum dos conjuntos seja vazio e que não exista intersecção entre eles. Esta condição é expressa formalmente a seguir:

$$
\begin{array}{r}
\Phi_{1} \neq \emptyset \\
\Phi_{2} \neq \emptyset \\
\Phi_{1} \cap \Phi_{2}=\emptyset
\end{array}
$$

onde $\Phi_{1}$ e $\Phi_{2}$ são os conjuntos de estados do GTT que satisfazem as fórmulas $\phi_{1}$ e $\phi_{2}$ respectivamente. 
É preciso esclarecer que a possibilidade de expressar esta propriedade não aumenta a expressividade da TCTL, apenas permite representar de um modo diferente as propriedades que podem ser calculadas de forma mais eficiente pelo verificador. Sendo assim inclui-se uma nova possibilidade na sintaxe da TCTL que poderia ser identificada como um operador de diferença temporal $\phi_{1}-\phi_{2} \sim c$.

Definição 3 (TCTL sintaxe). As fórmulas $\phi$ da TCTL são definidas indutivamente da seguinte forma:

$$
\phi:=p \mid \text { false }|\neg \phi| \phi_{1} \wedge \phi_{2}\left|\phi_{1} \rightarrow \phi_{2}\right| \exists\left[\phi_{1} U_{\sim_{c}} \phi_{2}\right]\left|\forall\left[\phi_{1} U_{\sim_{c}} \phi_{2}\right]\right| \phi_{1}-\phi_{2} \sim c
$$

onde $p \in P, c \in Q^{+}$, e $\sim$ é uma das seguintes relações binárias $<, \leq,=, \geq$, ou $>$.

A semântica do operador de diferença temporal é mostrada na tabela 3.2.

$$
\begin{aligned}
& s \vDash \phi_{1}-\phi_{2}<c \quad \text { sse } \quad \forall \omega=s_{0} \stackrel{a_{1}\left[l_{1}, u_{1}\right]}{\longrightarrow} s_{1} \ldots \stackrel{a_{n}\left[l_{n}, u_{n}\right]}{\longrightarrow} s_{n} \in\lceil\lambda\rceil \\
& \forall i, j \in[1 . . n] s_{i} \models \phi_{1}, s_{j}=\phi_{2}, i<j \text {, } \\
& \forall k, i<k<j, s_{k} \not \models \phi_{1}, \sum_{n=i+1}^{j} u_{n}<c \\
& s \models \phi_{1}-\phi_{2} \leq c \quad \text { sse } \quad \forall \omega=s_{0} \stackrel{a_{1}\left[l_{1}, u_{1}\right]}{\longrightarrow} s_{1} \ldots \stackrel{a_{n}\left[l_{n}, u_{n}\right]}{\longrightarrow} s_{n} \in\lceil\lambda\rceil \\
& \forall i, j \in[1 . . n] s_{i} \models \phi_{1}, s_{j}=\phi_{2}, i<j \text {, } \\
& \forall k, i<k<j, s_{k} \not \models \phi_{1}, \sum_{n=i+1}^{j} u_{n} \leq c \\
& s=\phi_{1}-\phi_{2}>c \quad \text { sse } \quad \forall \omega=s_{0} \stackrel{a_{1}\left[l_{1}, u_{1}\right]}{\longrightarrow} s_{1} \ldots \stackrel{a_{n}\left[l_{n}, u_{n}\right]}{\longrightarrow} s_{n} \in\lceil\lambda\rceil \\
& \forall i, j \in[1 . . n] s_{i} \models \phi_{1}, s_{j} \models \phi_{2}, i<j \text {, } \\
& \forall k, i<k<j, s_{k} \not \models \phi_{1}, \sum_{n=i+1}^{j} l_{n}>c \\
& s \models \phi_{1}-\phi_{2} \geq c \quad \text { sse } \quad \forall \omega=s_{0} \stackrel{a_{1}\left[l_{1}, u_{1}\right]}{\longrightarrow} s_{1} \ldots \stackrel{a_{n}\left[l_{n}, u_{n}\right]}{\longrightarrow} s_{n} \in\lceil\lambda\rceil \\
& \forall i, j \in[1 . . n] s_{i} \models \phi_{1}, s_{j}=\phi_{2}, i<j \text {, } \\
& \forall k, i<k<j, s_{k} \not \models \phi_{1}, \sum_{n=i+1}^{j} l_{n} \geq c
\end{aligned}
$$

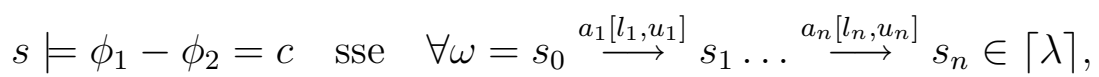

$$
\begin{aligned}
& \forall i, j \in[1 . . n] s_{i} \models \phi_{1}, s_{j} \models \phi_{2}, i<j \text {, } \\
& \forall k, i<k<j, s_{k} \not \models \phi_{1}, \sum_{n=i+1}^{j} l_{n}=\sum_{n=i+1}^{j} u_{n}=c
\end{aligned}
$$

Tabela 3.2: Semântica do operador de diferença temporal

\subsection{Um modelo finito para representar sistemas com tempo}

Uma característica do modelo usado para verificar propriedades é que este modelo deve preservar as propriedades que pretendemos verificar. No caso, se busca um 
modelo que preserve as propriedades de tempo ramificado, ou seja, que seja capaz de representar os diferentes futuros possíveis a partir de um determinado estado.

Como mencionou-se antes, para as RPT existem duas abordagens (ambas enumerativas) para representar sistemas de forma finita. A primeira delas é conhecida como grafo das regiões proposto pelo Alur, Courcoubetis e Dill (1990) e o segundo as classes de estados propostas por Berthomieu e Menasche (1983).

No primeiro caso um grafo temporizado é construído usando a abordagem do grafo das regiões. Este grafo temporizado é um grafo direcionado constituído por nós que representam as regiões, e arcos, que representam tanto o progresso do tempo quanto a evolução de uma transição discreta produto da ocorrência de eventos.

As regiões representam conjuntos de estados que apresentam o mesmo comportamento que é definido por três características: as proposições que caracterizam o estado, a parte inteira das variáveis relógio ativas nesse estado até o valor máximo da constante de tempo usada no modelo (ou da fórmula que pretende ser verificada) e finalmente a ordem das partes fracionárias das variáveis relógio ativas no estado.

Como foi mostrado em (ALUR; COURCOUBETIS; DILL, 1993) o número de regiões cresce exponencialmente com o número de relógios do modelo e com o maior valor da constante de tempo do modelo, e linearmente com a quantidade de elementos (nós e arestas) do modelo.

As classes de estados foram propostas em (BERTHOMIEU; MENASCHE, 1983), definidas como o conjunto de estados atingíveis a partir do estado inicial através de um esquema de disparos associados a uma determinada seqüência.

As classes de estados são determinadas por duas características: a marcação da rede, correspondente ao estado, e a junção de todos os domínios de disparo dos estados atingidos a partir do estado inicial disparando as transições na seqüência que caracteriza a própria classe de estado.

Este conceito mostrou-se restritivo demais e foi relaxado em (BERTHOMIEU; DIAZ, 1991) como sendo a junção de todos os valores de disparo possíveis a partir de uma determinada marcação. Outras definições de classes de estados podem ser encontradas na literatura, como por exemplo (BOUCHENEB; ALGER; BERTHELOT, 1993), todas elas semelhantes conceitualmente.

Usando a abordagem das classes de estado também é possível construir um grafo temporizado. Neste grafo os nós representam as classes de estado e os arcos 
as transições de uma classe de estado a outra, como resultado da ocorrência de um evento determinado.

Não existe uma análise da complexidade do algoritmo de geração das classes de estados, mas, levando-se em consideração que as estas só podem ser computadas a partir de RPT limitadas, podemos dizer que se a rede subjacente sem tempo de uma determinada RPT é $k$-limitada então o número de classes de estados geradas para esta rede estaria limitada pelo número de estados atingíveis da rede subjacente o que seria $P^{k} \times T$, que é o número máximo de marcações diferentes. Claro é, que como as RPT impõem mais restrições à evolução (se comparado com a rede de Petri subjacente não temporizada) o número de classes de estados pode ser bem menor.

O grafo temporizado, usando qualquer uma das abordagens aqui relacionadas, constitui uma abstração temporal do comportamento dos sistemas que estes representam. Sendo assim, os nós destes grafos representam um conjunto de estados equivalentes no seu comportamento e portanto são um tipo de "partição" do espaço de estados. Devido a isto as classes de estados são também denominadas em algumas publicações como espaço de estados abstratos.

Se o espaço de estados de um sistema fosse construído usando as duas abordagens acima duas partições diferentes deste espaço de estados seriam obtidas. Se essas construções preservam as mesmas propriedades então existe algum tipo de equivalência, mesmo que não seja uma relação bijetiva, entre as partições. Obviamente, se as duas cobrem todo o espaço de estado, quanto menor a partição, mais eficiente será o processo de verificação sobre uma estrutura gerada usando essa abordagem.

Uma bissimulação de abstração temporal é uma relação binária simétrica $B \subseteq Q \times Q$ entre estados de forma que para todos os estados $q_{1}, q_{2} \in Q,\left(q_{1}, q_{2}\right) \in B$ se,

$$
\begin{aligned}
& \forall \sigma \in \Sigma, \exists q_{1}^{\prime} \in Q\left|\left(q_{1} \stackrel{\sigma}{\rightarrow} q_{1}^{\prime}\right) \Rightarrow \exists q_{2}^{\prime}\right|\left(q_{2} \stackrel{\sigma}{\rightarrow} q_{2}^{\prime}\right) \wedge\left(q_{2}, q_{2}^{\prime}\right) \in B \\
& \forall \delta \in R^{+}, \exists q_{1}^{\prime}\left|\left(q_{1} \stackrel{\delta}{\rightarrow} q_{1}^{\prime}\right) \Rightarrow \exists q_{2}^{\prime} \in Q \exists \delta^{\prime} \in R^{+}\right|\left(q_{2} \stackrel{\delta^{\prime}}{\rightarrow} q_{2}^{\prime}\right) \wedge\left(q_{2}, q_{2}^{\prime}\right) \in B
\end{aligned}
$$

onde $\Sigma$ é o conjunto de eventos do sistema, ou seja, o alfabeto no caso dos autômatos, ou o conjunto das transições nas redes de Petri.

Se temos um modelo correto do sistema então temos uma bisimulação de abstração temporal entre os estados do sistema e os estados do modelo. Note que tanto o grafo das regiões quanto o grafo das classes de estado são bisimulações 
de abstração temporal, ainda que não necessariamente as que possuam a menor quantidade de partições.

Segundo Yovine (1996), a melhor partição (aquela que subdivide o espaço de estado no menor número de regiões ou classes de estados) deve cumprir as seguintes condições:

$$
\begin{aligned}
& s \in C_{1} \mid \exists s^{\prime} \in C_{2} . s \stackrel{\sigma}{\rightarrow} s^{\prime} \\
& s_{0}, \ldots, s_{n-1} \in C_{1} \mid \exists s_{n} \in C_{2} . s_{0} \stackrel{\epsilon}{\rightarrow} \ldots \stackrel{\epsilon}{\rightarrow} s_{n-1} \stackrel{\epsilon}{\rightarrow} s_{n}
\end{aligned}
$$

onde $C_{1}, C_{2}$ são regiões e $\epsilon$ representa um ifinitésimo de tempo.

Seja $\Omega$ a partição dos estados em $Q$ que agrupa os estados em regiões com comportamentos equivalentes. Então, $C_{1}, C_{2}, \ldots, C_{n} \in \Omega$ e $\forall q \in Q . \exists C \in \Omega \mid q \in C$.

A condição 3.2 acima estipula que a melhor partição possível é aquela que não possui transições que representam o progresso do tempo. Isto constitui uma forte evidencia de que a partição obtida usando a abordagem das classes de estado é melhor que a obtida usando a abordagem do grafo das regiões.

É precisamente o método de particionar o grafo das regiões em avanços unitários na parte inteira dos relógios mais a parte fracionária o que provoca que a complexidade do algoritmo seja dependente do valor da maior constante de tempo do sistema. Mesmo que a partição obtida através das classes de estados não seja ótima ${ }^{3}$ pelo menos não é diretamente dependente do valor da constante de tempo.

$\mathrm{Na}$ abordagem das classes de estado, a presença de grandes valores nos intervalos de tempo permitem - dependendo da estrutura da rede - um número maior de combinações das seqüências de disparo, aumentando assim o tamanho do grafo das classes de estados. De qualquer forma isto faz parte do comportamento do sistema modelado e portanto é pertinente ao seu processo de análise.

Por outro lado, é a partição excessiva do espaço de estados na abordagem do grafo das regiões justamente o que garante a conservação das propriedades de tempo ramificado. Yoneda e Ryuba (1998) encontraram uma condição suficiente que garante que as classes de estado preservem esse tipo de propriedade. As classes de estado que preservam as propriedades de tempo ramificado são conhecidas como classes de estados atômicas.

Os resultados experimentais obtidos em (YONEDA; RYUBA, 1998) mostraram

\footnotetext{
${ }^{3}$ Note que o fato de só conseguir representar restrições temporais convexas limita a possibilidade de se obter uma partição ótima.
} 
a diferença entre as partições do espaço de estado usando o grafo das regiões e as classes de estados atômicas computadas a partir do algoritmo proposto por eles.

\subsubsection{As classes de estado atômicas}

Em (BERTHOMIEU; VERNADAT, 2003) foram estabelecidas três condições que podem ser satisfeitas pelos grafos de classes de estados:

$$
\begin{array}{ll}
(E E) & (\exists t \in T)\left(\forall C_{1}, C_{2}\right)\left(C_{1} \stackrel{t}{\rightarrow} C_{2} \Leftrightarrow\left(\exists s \in C_{1}\right)\left(\exists s^{\prime} \in C_{2}\right)\left(s \stackrel{t}{\rightarrow} s^{\prime}\right)\right) \\
(A E) & (\exists t \in T)\left(\forall C_{2}\right)\left(C_{1} \stackrel{t}{\rightarrow} C_{2} \Rightarrow\left(\forall s \in C_{1}\right)\left(\exists s^{\prime} \in C_{2}\right)\left(s \stackrel{t}{\rightarrow} s^{\prime}\right)\right) \\
(E A) & (\exists t \in T)\left(\forall C_{2}\right)\left(C_{2} \stackrel{t}{\rightarrow} C_{1} \Rightarrow\left(\forall s \in C_{1}\right)\left(\exists s^{\prime} \in C_{2}\right)\left(s^{\prime} \stackrel{t}{\rightarrow} s\right)\right)
\end{array}
$$

onde $C_{1}$ e $C_{2}$ são classes de estados.

Como mostrado em (HADJIDJ; BOUCHENEB, 2008) a condição (EE) restringe demais a relação de transição entre classes de estados ao ponto de não ser respeitada pelos próprios grafos propostos em (BERTHOMIEU; VERNADAT, 2003).

Sendo assim a condição (EE) é substituída por uma combinação das condições denominadas $(S G)$ e $\left(E E_{r}\right) . E E=S G \wedge E E_{r}$, onde:

$(S G) \forall s \in S,\left(s \stackrel{t}{\rightarrow} s^{\prime}\right) \Rightarrow(\forall s \in C, C \in \Omega), \exists C^{\prime} \in \Omega$ tal que $s^{\prime} \in C^{\prime} \wedge C \stackrel{t}{\rightarrow} C^{\prime}$; $\left(E E_{r}\right)\left(C \stackrel{t}{\rightarrow} C^{\prime}\right) \Rightarrow \exists s \in C, \exists s^{\prime} \in C^{\prime}, s \stackrel{t}{\rightarrow} s^{\prime}$.

A condição $(S G)$ combinada com o fato de $C_{0}=\left\{s_{0}\right\}$ garante que cada estado concreto estará representado em ao menos uma classe de estado. A condição $\left(E E_{r}\right)$ evita que sejam conectadas duas classes de estados as quais não possuem estados concretos conectados.

A figura 3.1 mostra uma representação gráfica destas propriedades.
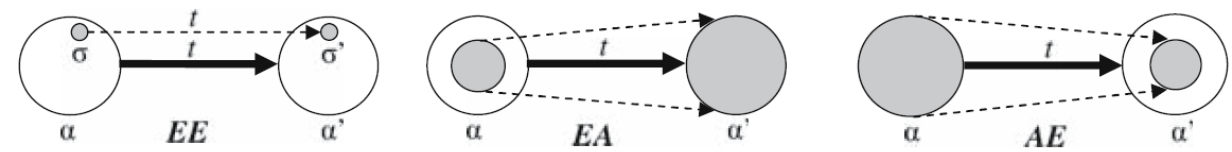

Figura 3.1: Propriedades (EE), (EA) e (AE).

Doravante neste trabalho vamos a adotar a seguinte definição (menos restrita) para as classes de estados. As classes de estado são uma representação de um conjunto de estados das RPT que possuem a mesma marcação, e compartilham as mesmas restrições temporais nas transições habilitadas. Isto é formalmente 
apresentado na seguinte definição:

Definição 4 (Classe de estado). Uma classe de estado $C$, de uma RPT é um par $(M, D)$ onde $M$ é a marcação e $D$ um conjunto de inequações denominadas de "dominio temporal"

As inequações em D são de dois tipos (BERTHOMIEU; DIAZ, 1991):

$$
D=\left\{\begin{aligned}
x_{i} \leq \theta_{i} \leq y_{i}, & x_{i}, y_{i} \in Q^{+}\left(\forall i \mid t_{i} \in \operatorname{enb}(M)\right) \\
\theta_{j}-\theta_{k} \leq z_{j k}, & z_{j k} \in Q^{+}\left(\forall j, k \mid j \neq k \text { e } t_{j}, t_{k} \in \operatorname{enb}(M)\right) ;
\end{aligned}\right.
$$

onde $\theta_{i}$ é o tempo de disparo da transição habilitada $t_{i}$, contado à partir do instante em que a marcação da classe foi alcançada.

Definição 5 (Grafo das classes de estados). O grafo das classes de estado de uma RPT é definido como um grafo cujos nós representam uma classe de estadouma partição do espaço de estados da RPT - e as arestas representam transições que satisfazem a condição (EE).

Segundo Yoneda e Ryuba (1998) uma condição suficiente para que as classes de estados preservem as propriedades de tempo ramificado é satisfazer (AE), além de (EE). Estes autores apresentam a seguinte definição para as classes de estado atômicas.

Definição 6 (Classes de estados atômicas). Uma classe de estado $\alpha$ é atômica se para qualquer estado $s \in \alpha$ e para qualquer classe de estado $\alpha^{\prime}$ tal que $\alpha \rightarrow \alpha^{\prime}$, existe um estado $s^{\prime}$ tal que $s \rightarrow s^{\prime}$.

Apesar da afirmação que (AE) é condição suficiente para que uma classe de estado seja atômica, o algoritmo proposto em (YONEDA; RYUBA, 1998) para calcular estas classes satisfaz (EE), (AE) e (EA). Como conseqüência obtém-se uma partição menos compacta do espaço de estado. Algoritmos que melhoraram de forma significativa este resultado foram propostos em (PENCZEK; PÓŁROLA, 2001); (BERTHOMIEU; VERNADAT, 2003) e (BOUCHENEB; RAKKAY, 2007).

Já o algoritmo proposto em (BERTHOMIEU; VERNADAT, 2003) satisfaz somente (EE) e (AE), além de trabalhar com uma definição de classe de estados menos restritiva, onde a seqüência geradora não é mais um fator excludente para agrupar estados. Esta abordagem do problema introduz de fato uma redução, pois permutações da mesma seqüencia são representadas pela mesma classe de estado sempre que a marcação alcançada for a mesma e que as restrições tempo- 
rais sejam satisfeitas. Geralmente esta estratégia consegue gerar grafos de classe de estados mais compactos.

A figura 3.2 traz o exemplo utilizado em (YONEDA; RYUBA, 1998) explicitando o método de geração das classes de estados atômicas, conjuntamente com seus grafos das classes de estados lineares (b) e atômicas (c).

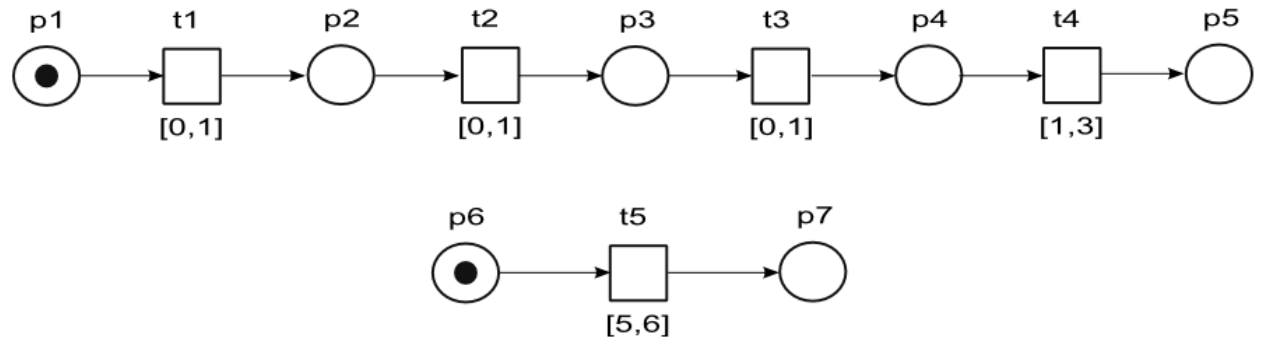

(a) Rede de Petri Temporizada

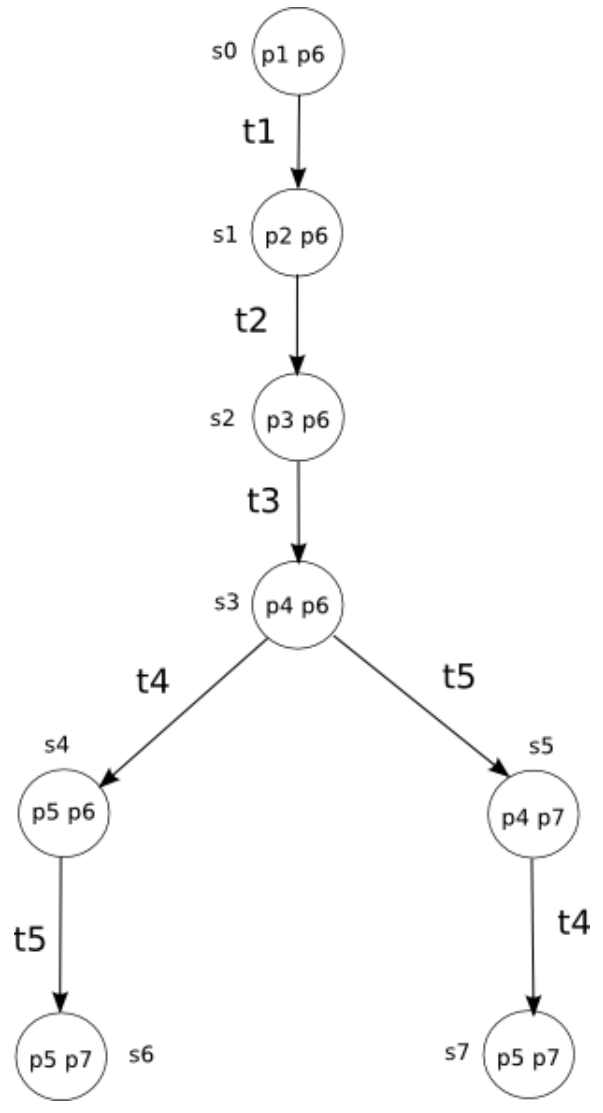

(b) Grafo das classes lineares

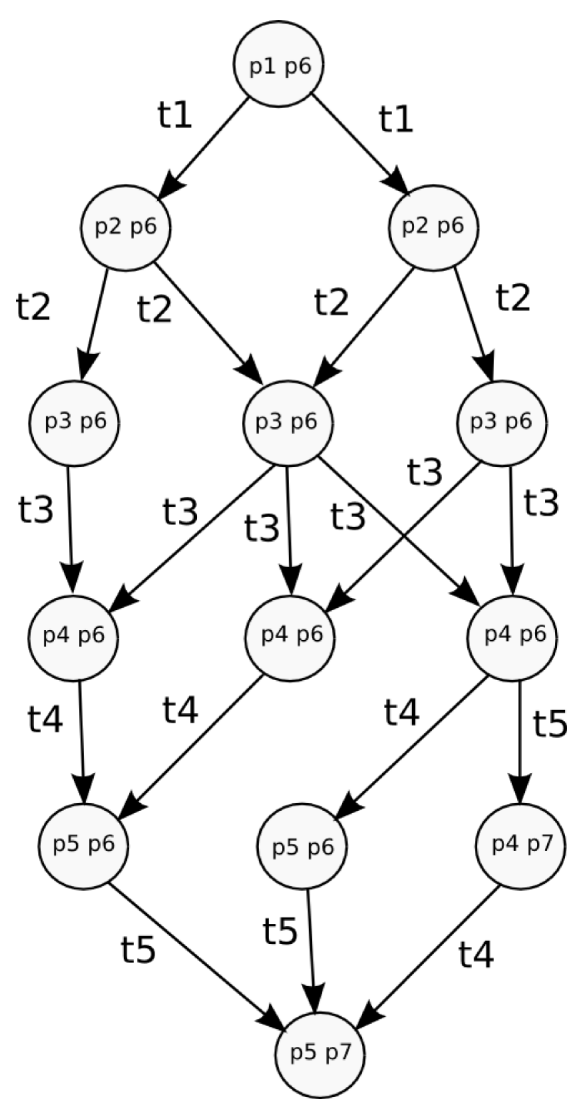

(c) Grafo das classes atômicas

Figura 3.2: Rede de Petri temporizada e seus grafos de classes de estados lineares e atômicas

A partir do trabalho de Yoneda e Ryuba (1998), outros métodos para a determinação das classes atômicas surgiram, como por exemplo (BERTHOMIEU; VERNADAT, 2003), (PENCZEK; PÓŁROLA, 2001), (BOUCHENEB; RAKKAY, 2007) e (HADJIDJ; BOUCHENEB, 2008). 
Especificamente em (PENCZEK; PÓŁROLA, 2001) foi proposta uma nova condição para os grafos de classe de estados denominada (U). Esta propriedade decorre do relaxamento da propriedade (AE) e justamente por isto as classes de estados que satisfazem (U) são denominadas de pseudo-atômicas.

Foi provado em (PENCZEK; PÓŁROLA, 2001) que os grafos pseudo-atômicos, que são aqueles onde todas as classes de estados satisfazem (U) e preservam as fórmulas $A C T L^{*}$, que é uma sub-linguagem da $C T L^{*}$.

Na ferramenta TINA é possível encontrar duas formas de fazer a geração das classes atômicas a partir de uma classe de estado linear denominada strong state class (SSC). Usando estas duas variantes de refinamento (opções -A e -U) as SSC são refinadas até as propriedades (EE) e (AE) ficarem restabelecidas.

A figura 3.3 mostra os grafos das classes de estados lineares e atômicas obtidas aplicando o algoritmo descrito em (FOYO; SILVA, 2008) e usando as partições geradas com as opções $-W$ e $-A$ da ferramenta TINA.

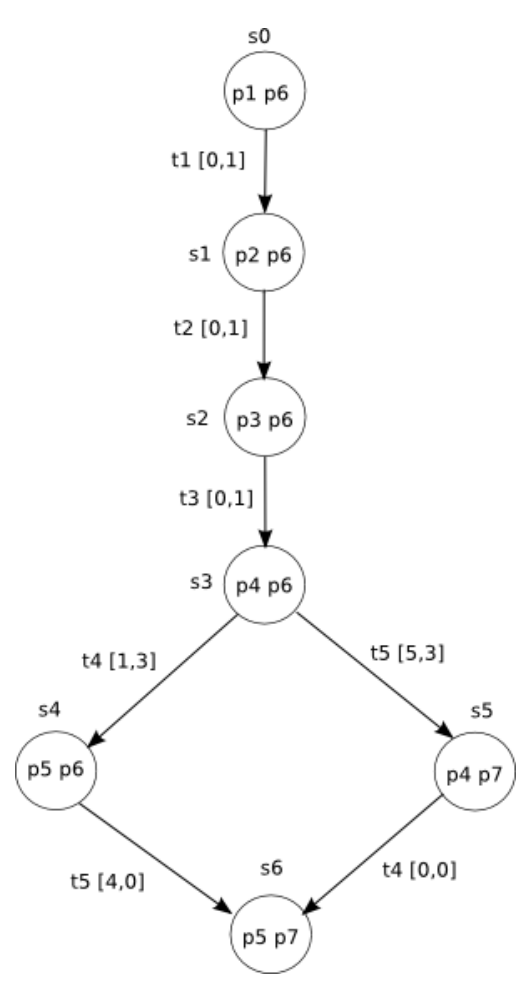

(a) Grafo das classes lineares

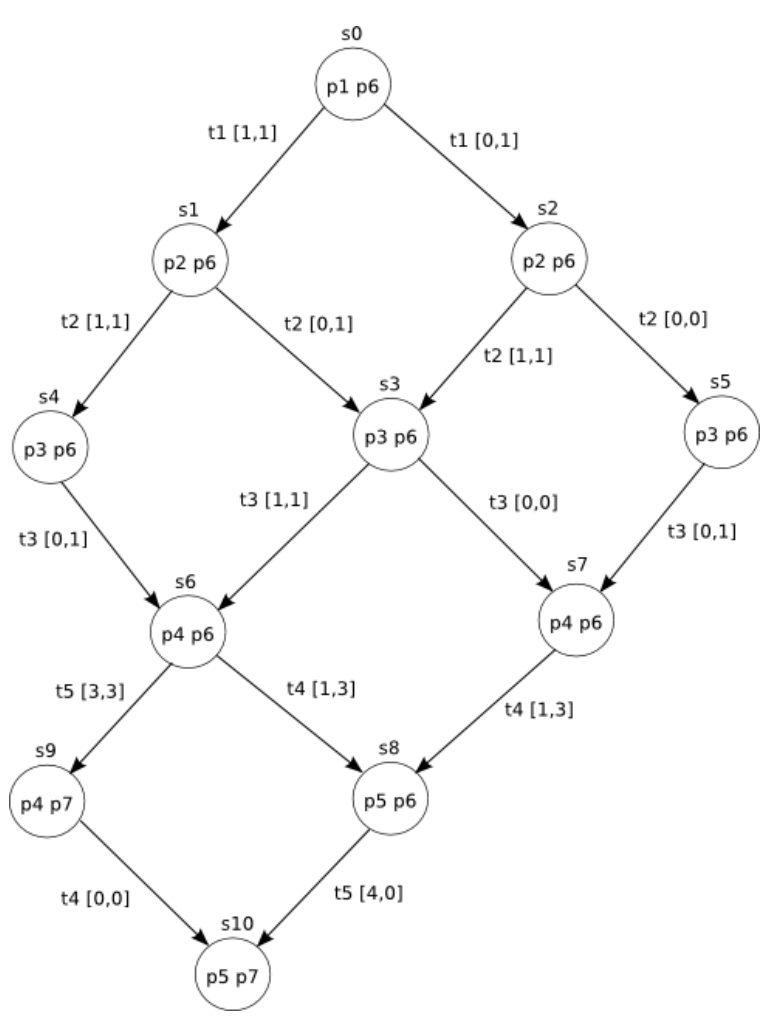

(b) Grafo das classes atômicas

Figura 3.3: Grafos de classes de estados lineares e atômicas para o exemplo da figura 3.2

No apêndice A podemos encontrar as tabelas com os resultados das classes de estados lineares A.1 e atômicas A.2. No apêndice A.1 mostra-se o cálculo dos incrementos de tempo para o grafo das classes de estados atômicas. 
Note que nem todas as soluções estão representadas neste grafo. Por exemplo, disparando $t 1 \mathrm{em} 0.73, t 2 \mathrm{em} 0.7$ e $t 3 \mathrm{em} 0.62$ pode-se atingir um estado em que tanto $t 4$ quanto $t 5$ são disparáveis. Um caminho como este não aparece no grafo das classes de estados atômicas da figura 3.2.

Quanto a eficiência na verificação das lógicas $C T L$ e $C T L^{*}$, podemos dizer que o algoritmo proposto em (BERTHOMIEU; VERNADAT, 2003) gera um número menor de classes (11 em lugar de 13) bem como o número menor de transições (15 em lugar de 19) e portanto resulta mais eficiente. A diferença decorre do fato de que não são representadas todas as soluções possíveis como mostrado anteriormente. Usando a abordagem do grafo das regiões para este mesmo exemplo o número de estados iria para 115 com 190 transições como mostram os resultados em (YONEDA; RYUBA, 1998).

Portanto, em uma comparação direta, a partição obtida usando as opções $-A$ e $-U$ na análise de atingibilidade ${ }^{4}$ garante uma maior eficiência na verificação das lógicas $C T L$ e $C T L^{*}$ que a partição obtida pelo algoritmo de Yoneda e Ryuba (1998). Por sua vez, este último é mais eficiente que a partição obtida pelo método do grafo das regiões.

Hadjidj e Boucheneb (2008) propuseram um outro tipo de partição que preserva as propriedades ramificadas que chamaram de atomic-CSZG. Esta partição é obtida através de refinamentos similares aos propostos em (YONEDA; RYUBA, 1998) e (BERTHOMIEU; VERNADAT, 2003). O ponto chave para a obter esta partição é uma contração do Grafo das Zonas de Estados Concretos (CC-CSZG pelas suas siglas em inglês), através de uma combinação convexa.

Independentemente da "qualidade" da partição do espaço de estados, as quatro abordagens existentes para a geração das classes de estado atômicas constroem um grafo onde as propriedades da lógica temporal ramificada são preservadas. Estas abordagens mostraram que, com os grafos gerados é possível verificar propriedades temporais qualitativas, mas nenhuma delas é capaz de verificar propriedades quantitativas. Para conseguir verificar propriedades temporais quantitativas é preciso determinar a duração (mínima e máxima) entre cada uma das transições de estado do sistema (WANG; DENG; XU, 2000).

A primeira idéia para determinar a duração das transições de estado e portanto verificar propriedades temporais quantitativas foi baseada na determinação da diferença de tempo entre os domínios temporais das classes de estados. Apa-

\footnotetext{
${ }^{4}$ Para o exemplo da figura 3.2 as classes de estados obtidas usando ambas opções são exatamente iguais.
} 
rentemente esse método funcionou para as classes lineares e portanto constitui a base das verificações temporais em (WANG; DENG; XU, 2000) e (BERTHOMIEU; RIBET; VERNADAT, 2004).

A figura 3.4 mostra una rede de Petri temporizada usada como exemplo da técnica para determinar os intervalos de tempo globais em (WANG; DENG; XU, $2000)$.

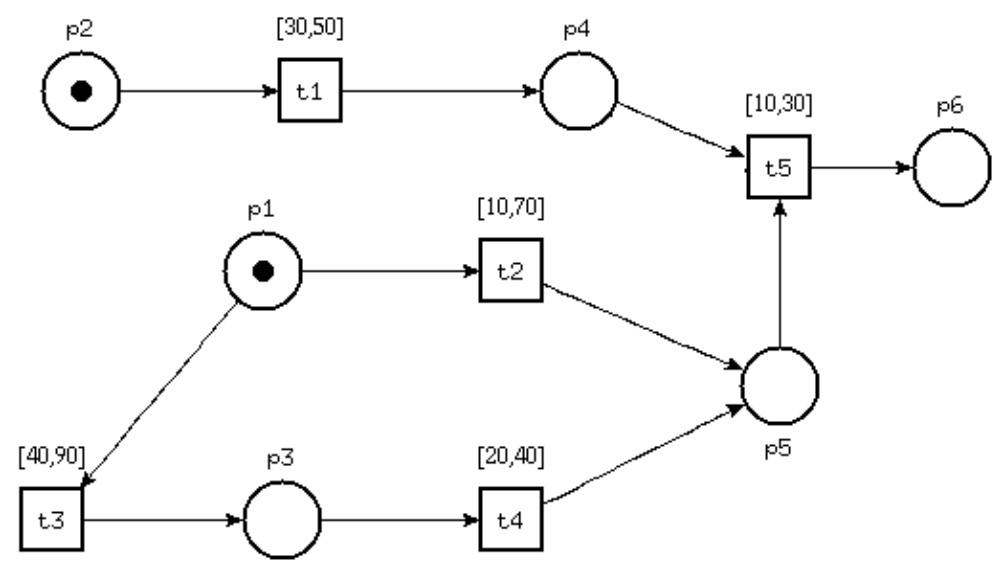

Figura 3.4: Exemplo de RPT modelando um sistema com concorrência, conflito e sincronização.

As estruturas resultantes da aplicação dos métodos apresentados em (WANG; DENG; XU, 2000) e (BERTHOMIEU; RIBET; VERNADAT, 2004) são mostradas nas figuras 3.5 e 3.6 respectivamente.

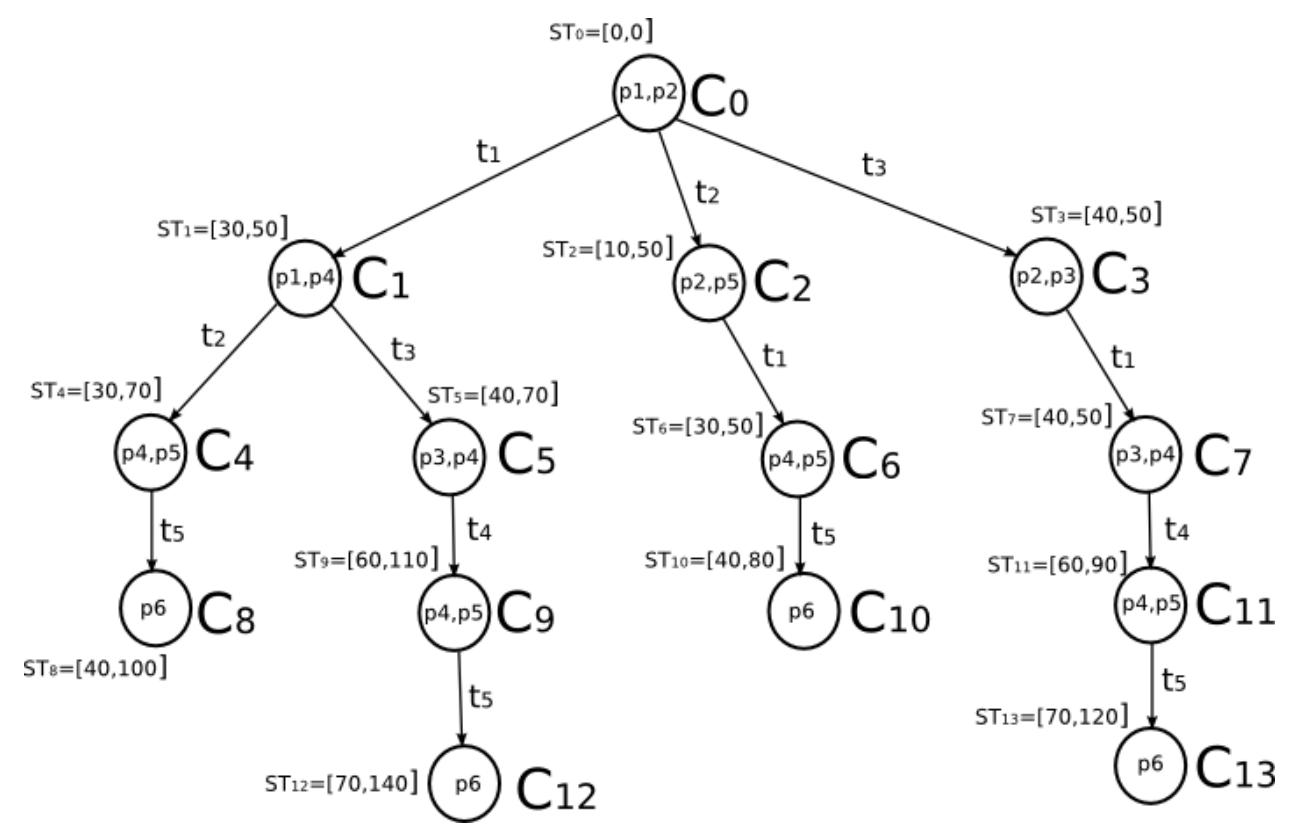

Figura 3.5: Árvore de atingibilidade com seus respectivos intervalos de tempo global da fig. 3.4 


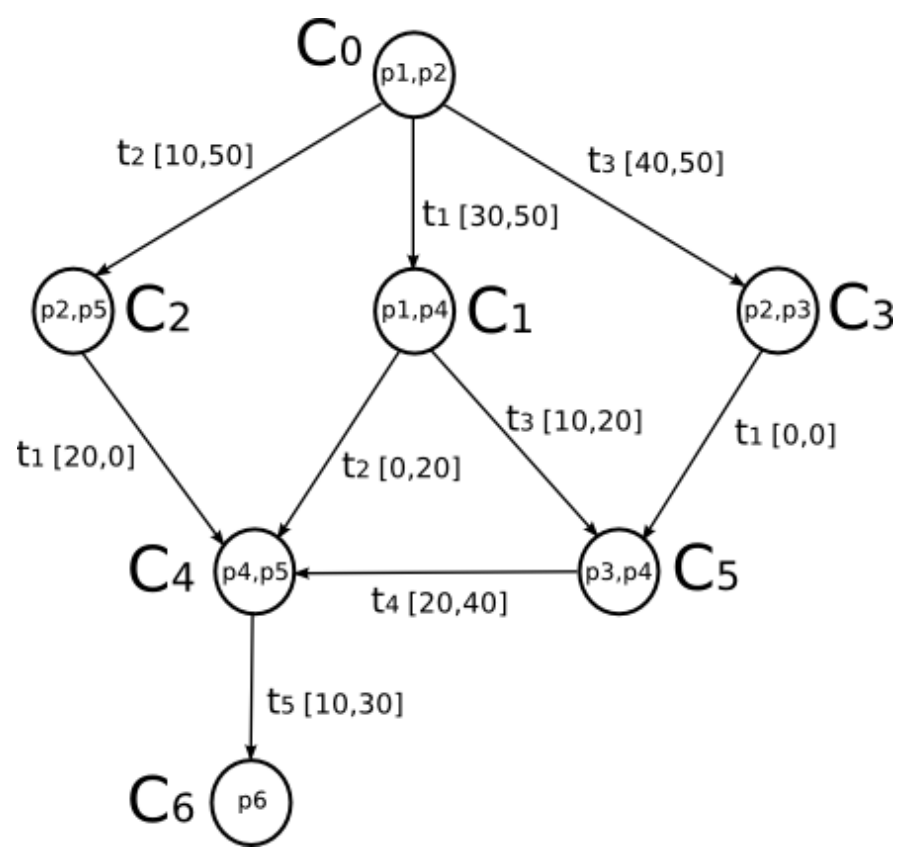

Figura 3.6: LTS construído a partir das classes de estados lineares usando TINA

A estrutura obtida a partir das classes de estado atômicas computadas pelo TINA usando a abordagem da diferença dos domínios das classes de estados proposta em (FOYO; SILVA, 2008) é bastante similar à mostrada na figura 3.6. O fato é que dependendo da estrutura da rede e da amplitude dos intervalos estáticos de disparo das transições $(L F T-E F T)$, os métodos anteriores podem não funcionar ou levar a valores incorretos do tempo, e portanto a uma validação equivocada de uma determinada fórmula temporal.

Como neste trabalho estamos interessados na verificação da lógica TCTL é preciso que as partições que preservam as propriedades de tempo ramificado permitam calcular de forma correta o tempo transcorrido em qualquer caminho através da mesma.

A partição proposta em (PENCZEK; PÓŁROLA, 2001) não será considerada porque preserva apenas um sub-conjunto da CTL.

Os grafos das classes de estados atômicas são computados a partir de refinamentos de grafos de classes de estados que preservam as propriedades lineares dos modelos em RPT. Por sua vez estes são construídos usando um algoritmo do tipo $\operatorname{succ}\left(C, t_{f}\right)=C^{\prime}$ apresentado em (HADJIDJ; BOUCHEnEB, 2008). Por construção, estes grafos preservam as condições (EE) e (EA) sendo que todos os caminhos possíveis no modelo em RPT estão representados na partição do espaço de estados. 
Em alguns grafos de classe de estados atômicos apenas as marcações são preservadas. Tal é o caso do ASCG (BERTHOMIEU; VERNADAT, 2003). Isto permite gerar grafos mais compactos e melhorar a complexidade dos algoritmos de geração das classes de estados. Portanto conduzem a uma melhor complexidade na verificação da $C T L$ e $C T L^{*}$.

O processo de refinamento para transformar um grafo que preserva as propriedades lineares em um que preserva as de tempo ramificado é feito através da determinação da partição maximalmente granular (coarsest partition). Neste processo de refinamento a condição (EA) não é mais satisfeita, e alguns caminhos válidos no modelo em RPT não são mais representados na partição. Devido a isto, o cálculo dos incrementos de tempo mínimo e máximo nas transições de estados pode levar a resultados incorretos na verificação de propriedades quantitativas.

A abordagem apresentada em (HADJIDJ; BOUCHENEB, 2008) usa o CSZG como grafo intermediário na obtenção de um grafo de classes de estados atômicas. Apesar do CSZG preservar todos os caminhos do modelo em RPT e a condição (EA), o processo de contração das classes através da combinação convexa impossibilita o uso desta na determinação dos incrementos de tempo nas transições de forma correta. Assim, mesmo que a operação de contração dos domínios não seja feita o método de refinamento usado não garante a manutenção da condição (EA).

O algoritmo proposto em Yoneda e Ryuba (1998) para dividir as classes lineares até obter uma partição do espaço de estado onde todas as classes de estados sejam atômicas gera um grafo que satisfaz as condições (EE), (EA), (AE) assim como a preservação de todos os caminhos do modelo em RPT.

Entretanto, esta técnica tem vários inconvenientes:

- Cada classe de estado é definida a partir das transições que foram disparadas desde o estado inicial até chegar à transição alvo, além das transições habilitadas nesse estado. Isto faz com que as estruturas que representam estas classes sejam cada vez maiores a medida que representam caminhos mais longos.

- Para calcular o tempo transcorrido em cada transição é necessário resolver um sistema de inequações (com um número cada vez maior de variáveis) o que torna o método impraticável para sistemas reais.

- Como cada ocorrência da mesma transição é representada por uma variável diferente, o grafo das classes de estados pode crescer infinitamente mesmo 
quando a rede subjacente é limitada (HADJIDJ; BOUCHENEB, 2008).

A partir desta análise pode-se concluir que os algoritmos existentes para o cálculo das classes de estado atômicas, embora preservem as propriedades de tempo ramificado, não permitem a verificação de propriedades quantitativas.

\subsubsection{As classes de estados com restrições}

Em todas as abordagens analisadas na seção anterior o conjunto de estados representados nas classes de estados tem o seu domínio temporal representado por DBMs. Isto limita a representação de regiões temporais convexas embora seja vantajoso em relação as CDDs no que diz respeito à complexidade do cálculo das classes de estados (BERTHOMIEU; VERNADAT, 2006).

No entanto, uma vez que os algoritmos existentes para gerar as classes de estado atômicas não nos permitem obter resultados satisfatórios para a verificação de propriedades quantitativas para lógicas de tempo ramificado, é preciso investigar outras alternativas.

Nesta subseção será apresentada uma discussão de alguns aspectos da lógica de tempo ramificado através de um exemplo. A figura 3.7 mostra os tempos válidos para o disparo de cada uma das transições na RPT da figura 3.2.

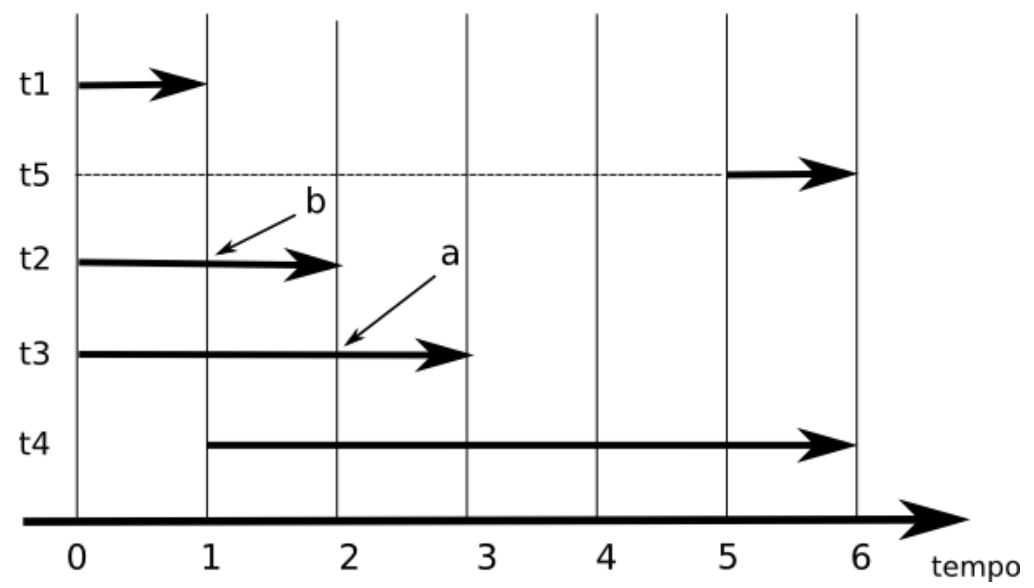

Figura 3.7: Carta de tempo da RPT da figura 3.2

A coincidência temporal entre transições concorrentes, como é o caso de $t 4 \mathrm{e}$ $t 5$, é a causa do comportamento temporal ramificado. Note que $t 4$ e $t 5$ poderão acontecer em qualquer ordem dependendo do momento em que tenham acontecido $t 1, t 2$ e $t 3$. O marcador $a$ sinaliza o tempo mínimo em que deve ocorrer $t 3$ para que $t 4$ e $t 5$ possam acontecer em qualquer ordem. O marcador $b$ sinaliza o tempo mínimo que deve demorar $t 2$ para que $t 4$ e $t 5$ possam acontecer em qualquer 
ordem. Este comportamento temporal ramificado é claramente mostrado através das diferenças dos grafos lineares e ramificados das figuras 3.2 e 3.3 .

De qualquer forma, o marcador $b$ não tem nenhuma influência no comportamento ramificado, mas o marcador $a$ contém informação suficiente para identificar o comportamento ramificado. Isto pode ser aproveitado para gerar uma partição do espaço de estados que preserve as propriedades de tempo ramificado de modo mais eficiente, ou seja, com o menor número de classes possíveis.

Esta situação, mesmo sendo freqüente, não acontece em todos os domínios temporais. Portanto, a nossa proposta é substituir o domínio temporal com todas as transições disparadas a partir do estado inicial pelo domínio temporal apenas com as transições habilitadas nesse estado, e aplicar uma restrição temporal à transição responsável pelo comportamento ramificado.

A restrição temporal é similar à restrição implícita referenciada em (YONEDA; RYUBA, 1998). Voltando ao exemplo da figura 3.2 a restrição usada na divisão da classe $s_{3}$, foi: $\theta_{3}-2 \geq v$ e $\theta_{3}-2<v$ onde $v$ representa o momento em que o estado $s_{0}$ foi atingido. Note que esta restrição é sinalizada pelo marcador $a$ na figura 3.7. Na divisão da classe $s_{2}$ é usada a restrição: $\theta_{2}-1 \geq v$ e $\theta_{2}-1<v$ que é a mesma sinalizada pelo marcador $b$ na figura 3.7 .

Como as restrições são referentes a estados ancestrais do estado que deve ser dividido - tendo como base transições habilitadas nesse estado - a restrição pode ser aplicada diretamente na inequação do domínio temporal da transição em questão.

A seção a seguir mostra como pode ser obtido o grafo das classes de estados com restrições.

\subsubsection{O algoritmo de geração das classes de estados com restrições}

Como foi colocado na seção anterior, as classes de estados com restrições devem preservar as propriedades ramificadas. Isto pode ser garantido através de alguma condição suficiente, como acontece no casos das classes de estados atômicas.

Como ponto de partida para a geração das classes de estados com restrições utilizaremos o SSCG proposto em (BERTHOMIEU; VERNADAT, 2003) (denominado CSZG em (HADJIDJ; BOUCHENEB, 2008)). A proposição a seguir é utilizada para gerar o sucessor de uma classe de estado na sua forma canônica.

Proposição 7 (Determinação do domínio para uma classe sucessora). 
Seja $s=(M, D)$ uma classe de estado na sua forma canônica e $t_{j}$ uma transição habilitada pela marcação $M$.

- $t_{j}$ é disparável em s sse $\operatorname{EFT}\left(t_{j}\right)-D_{t_{j} o} \leq \operatorname{Min}_{u \in e n b(M)}\left(\operatorname{LFT}(u)-D_{\text {ou }}\right)$.

- se $t_{j}$ é disparável em s então o intervalo de disparo é: $I\left(t_{j}\right)=\left[\max \left(0, \operatorname{EFT}\left(t_{j}\right)-D_{t_{j} o}\right), \operatorname{Min}_{u \in e n b(M)}\left(\operatorname{LFT}(u)-D_{\text {ou }}\right)\right]$.

- a classe sucessora de s pelo disparo da transição t $t_{j}$ no intervalo de disparo $I\left(t_{j}\right), s \stackrel{t_{j}, I\left(t_{j}\right)}{\longrightarrow} s^{\prime}, s^{\prime}=\left(M^{\prime}, D^{\prime}\right)$ é computada como:

$$
\begin{aligned}
& M^{\prime}=M-\operatorname{Pre}\left(t_{j}\right)+\operatorname{Pos}\left(t_{j}\right) ; \operatorname{now}\left(M^{\prime}\right)=\left\{t \in \operatorname{enb}\left(M^{\prime}\right) \mid t=t_{f} \vee \exists p, M^{\prime}(p)-\operatorname{Pos}\left(p, t_{f}\right) \leq \operatorname{Pre}(p, t)\right\} ; \\
&\left.\forall t \in \operatorname{enb}\left(M^{\prime}\right), D_{t o}^{\prime}\right)\left\{\begin{array}{r}
0 \text { se } t \in \text { new }\left(M^{\prime}\right) ; \\
\operatorname{Min}_{u \in \operatorname{enb}(M)}\left(\operatorname{LFT}(u)+D_{t u}\right) \text { nos casos restantes }
\end{array}\right. \\
& D_{o t}^{\prime}=\left\{\begin{array}{r}
\max \left(0, E F T\left(t_{j}\right)-D_{t_{j} t}\right) \text { nos casos restantes } \\
0 \text { se } t=t^{\prime} \text { ou } t \wedge t^{\prime} \in \text { new }\left(M^{\prime}\right) ;
\end{array}\right. \\
& \forall\left(t, t^{\prime}\right) \in \operatorname{enb}\left(M^{\prime}\right)^{2}, D_{t t^{\prime}}^{\prime}=\left\{\begin{array}{r}
D_{t o}^{\prime}-D_{o t^{\prime}}^{\prime} \text { se } t \vee t^{\prime} \in \text { new }\left(M^{\prime}\right) ; \\
D_{t t^{\prime}} \text { nos casos restantes }
\end{array}\right.
\end{aligned}
$$

onde $D$ é uma $D B M$ de ordem $|e n b(M) \cup\{o\}|$ e o representa o valor zero.

Esta proposição é utilizada para construir um algoritmo que gera o sucessor de uma classe de estado com complexidade $O\left(n^{2}\right)$, onde $n$ é o número de transições habilitadas em $M$.

Como os intervalos estáticos nas transições admitem $\infty$ como LFT é necessário uma normalização como a proposta em (HADJIDJ; BOUCHENEB, 2008). O teorema 4 em (HADJIDJ; BOUCHENEB, 2008) garante que o CSZG normalizado continua sendo um espaço abstrato, que é finito se a rede de Petri subjacente é limitada e que preserva as propriedades lineares do modelo. Também é garantido pela própria definição do CSZG que todos os estados concretos do modelo em TPN estão representados em pelo menos uma classe de estado (definição $8 \mathrm{em}$ (HADJIDJ; BOUCHENEB, 2008)).

Uma vez construído o CSZG, uma técnica de refinamento da partição precisa ser utilizada para construir um espaço abstrato que preserve as propriedades ramificadas. Além disso, esta técnica deve garantir que todos os caminhos admissíveis no modelo da RPT estejam representados na partição do espaço de 
estados e que os incrementos de tempo entre as transições de estados possam ser computadas de forma correta.

Em (YONEDA; RYUBA, 1998) foi estabelecida a propriedade de "atomicidade" como suficiente para um grafo preservar as propriedades de tempo ramificado. Mesmo assim, esta propriedade é restritiva demais para gerar grafos que preservem estas propriedades.

As propriedades de tempo ramificado são preservadas em grafos de classes de estados que têm estrutura de árvore, onde estão representados todos os possíveis "futuros" a partir de um estado inicial. Do ponto de vista do comportamento de uma RPT, todos os "futuros" podem ser representados em um grafo de classes de estados se todos os estados concretos, pertencentes a cada classe de estados, possuem o mesmo conjunto de transições disparáveis.

Definição 8 (Classe de estados estáveis). Uma classe de estado é dita estável se todos os estados concretos que pertencem a ela possuirem as mesmas transições disparáveis. Formalmente:

$$
\forall s \in C, C \in \Omega \Upsilon(s)=\Upsilon(C)
$$

Definição 9 (Grafo de classes de estados estável). Um grafo de classe de estados $G$ é estável se todas as classes de estados em $G$ são estáveis.

Diferentemente dos algoritmos para calcular os grafos que preservam as propriedades de tempo ramificado em (YONEDA; RYUBA, 1998), (BERTHOMIEU; VERNADAT, 2003), (HADJIDJ; BOUCHENEB, 2008), o algoritmo aqui proposto vai dividir as classes do CSZG que não sejam estáveis assim estas forem obtidas. Desta forma evita-se ter de continuar aplicando as restrições aos sucessores das classes divididas. O grafo resultante deste algoritmo será denominado como CSCG.

O algoritmo é o seguinte:

1. Inicializa a lista de estados a processar com o estado inicial;

2. Tira um estado da lista e computa as classes sucessoras, usando a proposição 7

3. Verifica se alguma das classes geradas não é estável usando a proposição 10;

4. Se existe alguma classe não estável, detecta a restrição a ser usada e divide esta classe em duas. 
5. Computa os incrementos mínimos e máximo de cada transição de estado;

6. Determina se alguma das classes geradas possui um equivalente entre as já processadas, se achar alguma, se deve agregá-las.

7. As classes computadas são colocadas na lista;

8. Repete os passos 2 a 7 até que não existam mais sucessores a calcular.

Proposição 10 (Identificação de classes não estáveis). Seja $s_{i}=\left(M^{\prime}, D^{\prime}\right)$ uma classe de estados tal que $s_{i-1} \stackrel{I\left(t_{j}\right)}{\longrightarrow} s_{i}, s_{i}$ é não estável ou instável sse satisfaz uma das seguintes condições:

$$
\begin{aligned}
& \exists t, t^{\prime} \in \operatorname{enb}\left(M^{\prime}\right), t \in \operatorname{new}\left(M^{\prime}\right) \text { tal que : } \\
& \qquad \begin{aligned}
D_{o t^{\prime}}^{\prime} & <\operatorname{EFT}\left(t^{\prime}\right)-\operatorname{LFT}(t)<D_{t^{\prime} o}^{\prime} \\
D_{o t^{\prime}}^{\prime} & <\operatorname{LFT}\left(t^{\prime}\right)-\operatorname{EFT}(t)<D_{t^{\prime} o}^{\prime}
\end{aligned}
\end{aligned}
$$

Se a condição 3.4 é satisfeita $t^{\prime}$ pode ou não ser disparável, dependendo do tempo em que esta foi recém habilitada em uma classe antecessora de $s_{i}$. Se a condição 3.5 é satisfeita $t$ pode ou não ser disparável, dependendo do tempo em que a transição $t^{\prime}$ foi recém habilitada em uma classe antecessora a $s_{i}$.

Os valores das restrições para cada caso detectado usando a proposição 10 são mostrados nas tabelas 3.3 e 3.4. Para determinar a restrição deve-se procurar a classe onde a transição $t^{\prime}$ foi recém habilitada pela última vez. Na formulação das restrições foi considerado que $t^{\prime}$ foi recém habilitada pela última vez em um estado antecessor de $s_{i}$, o qual denominaremos $s_{k}$.

Além das restrições, as tabelas 3.3 e 3.4 mostram os valores $\left[\Delta_{\min }, \Delta_{\max }\right]$ que são usados na correção dos domínios divididos e o intervalo $I\left(t_{j}\right)$ que é o intervalo de disparo da transição $t_{j}$ para cada uma das novas classes resultantes do processo de divisão.

\begin{tabular}{|l|c|c|}
\hline Condição 3.4 & \multicolumn{2}{|c|}{$\sigma=s_{k} \rightarrow \ldots \rightarrow s_{i-1} \stackrel{t_{j}}{\rightarrow} s_{i}$} \\
\hline Restrição & $s_{i}-s_{k} \geq E F T\left(t^{\prime}\right)-L F T(t)$ & $s_{i}-s_{k}<E F T\left(t^{\prime}\right)-L F T(t)$ \\
\hline$\left[\Delta_{\text {min }}, \Delta_{\text {max }}\right]$ & $E F T\left(t^{\prime}\right)-L F T(t)-D_{o t^{\prime}}^{\prime}$ & $E F T\left(t^{\prime}\right)-D_{t^{\prime} o}^{\prime}-L F T(t)-\epsilon$ \\
\hline$s_{i-1} \stackrel{I\left(t_{t^{\prime}}\right)}{\stackrel{(t}{(})} s_{i a}$ & $E F T\left(t_{j}\right)-D_{t_{j} o}$ & $\operatorname{Min}_{u \in \operatorname{enb}(M)}\left(L F T(u)-D_{o u}\right)$ \\
\hline$s_{i-1} \stackrel{I\left(t_{j}\right)}{\longrightarrow} s_{i b}$ & $E F T\left(t_{j}\right)-D_{t_{j} o}$ & $\operatorname{Min}_{u \in \operatorname{enb}(M)}\left(L F T(u)-D_{o u}\right)$ \\
\hline
\end{tabular}

Tabela 3.3: Restrição usada na divisão da classe $s_{i}$ para a condição 3.4 


\begin{tabular}{|l|c|c|}
\hline Condição 3.5 & \multicolumn{2}{|c|}{$\sigma=s_{k} \rightarrow \ldots \rightarrow s_{i-1} \stackrel{t_{j}}{\rightarrow} s_{i}$} \\
\hline Restrição & $s_{i}-s_{k}>L F T\left(t^{\prime}\right)-E F T(t)$ & $s_{i}-s_{k} \leq L F T\left(t^{\prime}\right)-E F T(t)$ \\
\hline$\left[\Delta_{\min }, \Delta_{\max }\right]$ & $L F T\left(t^{\prime}\right)-E F T(t)-D_{o t^{\prime}}^{\prime}+\epsilon$ & $L F T\left(t^{\prime}\right)-D_{t^{\prime} o}^{\prime}-E F T(t)$ \\
\hline$s_{i-1} \stackrel{I\left(t_{j}\right)}{\longrightarrow} s_{i a}$ & $D_{o t^{\prime}}^{\prime}-D_{o t^{\prime}}+\Delta_{\min }$ & $\operatorname{Min}_{u \in e n b(M)}\left(L F T(u)-D_{o u}\right)$ \\
\hline$s_{i-1} \stackrel{I\left(t_{j}\right)}{\longrightarrow} s_{i b}$ & $\operatorname{Max}\left(0, E F T\left(t_{j}\right)-D_{t_{j} o}\right)$ & $D_{t^{\prime} o}^{\prime}-D_{o t^{\prime}}+\Delta_{\max }$ \\
\hline
\end{tabular}

Tabela 3.4: Restrição usada na divisão da classe $s_{i}$ para a condição 3.5

Os domínios das novas classes $s_{i a}$ e $s_{i b}$ são computados utilizando a seguinte proposição:

\section{Proposição 11 (Determinação dos domínios para classes divididas).} Dado o domínio da classe não estável $s_{i}=\left(M^{\prime}, D^{\prime}\right)$, devido a transição $t^{\prime} \in$ enb $\left(M^{\prime}\right), t^{\prime} \notin$ new $\left(M^{\prime}\right)$, e os valores de $\Delta_{\min }$ e $\Delta_{\max }$ segundo a condição que determinou que a mesma não é estável os domínios das novas classes $s_{i a}$ e $s_{i b}$, $D^{a}$, e $D^{b}$ são obtidos da seguinte forma:

$$
\begin{aligned}
& \forall t \in \operatorname{enb}\left(M^{\prime}\right), \quad D_{t o}^{a}=D_{t o}^{\prime} \\
& D_{o t}^{a}=\left\{\begin{aligned}
D_{o t}^{\prime}+\Delta_{\text {min }} & \text { se } D_{t t^{\prime}}^{\prime}=D_{t^{\prime} t}^{\prime}=0 \text { ou } t=t^{\prime} \\
D_{o t}^{\prime} & \text { nos casos restantes }
\end{aligned}\right. \\
& \forall\left(t, t^{\prime \prime}\right) \in \operatorname{enb}\left(M^{\prime}\right)^{2}, \quad D_{t t^{\prime \prime}}^{a}=\left\{\begin{aligned}
0 & \text { se } t=t^{\prime \prime} \\
\operatorname{Min}\left(D_{t t^{\prime \prime}}^{\prime}, D_{t o}^{a}-D_{o t^{\prime \prime}}^{a}\right) & \text { nos casos restantes }
\end{aligned}\right. \\
& \forall t \in \operatorname{enb}\left(M^{\prime}\right), \quad D_{t o}^{b}=\left\{\begin{array}{cl}
D_{t o}^{\prime}+\Delta_{\max } & \text { se } D_{t t^{\prime}}^{\prime}=D_{t^{\prime} t}^{\prime}=0 \text { ou } t=t^{\prime} \\
D_{\text {to }}^{\prime} & \text { nos casos restantes }
\end{array}\right. \\
& \forall t \in \operatorname{enb}\left(M^{\prime}\right), \quad D_{o t}^{b}=D_{o t}^{\prime} \\
& \forall\left(t, t^{\prime \prime}\right) \in \operatorname{enb}\left(M^{\prime}\right)^{2}, \quad D_{t t^{\prime \prime}}^{b}=\left\{\begin{aligned}
0 & \text { se } t=t^{\prime \prime} ; \\
\operatorname{Min}\left(D_{t t^{\prime \prime}}^{\prime}, D_{t o}^{b}-D_{o t^{\prime \prime}}^{b}\right) & \text { nos casos restantes }
\end{aligned}\right.
\end{aligned}
$$

O incremento de tempo global, necessário para calcular os tempos mínimos e máximos em uma seqüência, em cada uma destas transições é computada usando a proposição 12, uma vez que os domínios temporais das classes divididas são determinados. 
ses de estados com os seus domínios temporais $D$ e $D^{\prime}$ na sua forma canônica, onde $s \stackrel{t_{j}}{\longrightarrow} s^{\prime}$. O incremento de tempo mínimo e máximo entre a s e $s^{\prime}$, denotado como $l\left(t_{j}\right)$ e $u\left(t_{j}\right)$, respectivamente, é computado da seguinte forma:

$$
\begin{aligned}
\forall t \in \operatorname{enb}(M), \quad \delta_{t} & = \begin{cases}E F T(t)-D_{o t} & \text { se } t=t_{j} ; \\
D_{o t}^{\prime}-D_{o t} & \text { se } t \in \operatorname{enb}\left(M^{\prime}\right) \\
0 & \text { nos casos restantes }\end{cases} \\
\Delta_{t} & = \begin{cases}D_{t o}^{\prime}-D_{t o} & \text { se } t \in \operatorname{enb}\left(M^{\prime}\right) \\
L F T(t)-D_{t o} & \text { nos casos restantes }\end{cases} \\
l\left(t_{j}\right)=\max \left(\delta_{t}\right), & u\left(t_{j}\right)=\min \left(\Delta_{t}\right)
\end{aligned}
$$

A definição usada para determinar as classes equivalentes é similar as apresentadas em (BERTHOMIEU; MENASCHE, 1983), (BERTHELOT, 1994), (BERTHOMIEU; RIBET; VERNADAT, 2004), (BERTHOMIEU; VERNADAT, 2006), (BOUCHENEB; RAKKAY, 2007).

Definição 13 (Equivalência entre classes de estados com restrições). Duas classes de estados $C_{w}=\left(M_{w}, \Delta_{w}\right)$ e $C_{w^{\prime}}=\left(M_{w^{\prime}}, \Delta_{w^{\prime}}\right)$ são equivalentes se e somente se $M_{w}=M_{w^{\prime}}$, e $\Delta_{w}=\Delta_{w^{\prime}}$. Esta relação de equivalência é denotada como $C_{w} \cong C_{w^{\prime}}$.

Inicialmente a lista dos estados vivos contém apenas o estado inicial. O processamento de cada estado da lista é feito seguindo o método de ramificação e poda (branch and bound) o qual minimiza a quantidade de memória usada pelo stack em comparação com o método de busca em profundidade.

Cada vez que é atingido um estado onde todas as transições vizinhas são recém habilitadas, este é colocado na lista - desde que já não esteja nela nem seja equivalente a um estado já processado. Desta forma reduze-se o impacto do tratamento das classes equivalentes.

Lema 14 (Completeza do CSSG). O CSCG contém todos os estados concretos assim como todos os caminhos admissíveis do modelo da RPT.

Prova O CSCG utiliza como ponto de partida o CSZG, que contém todos os estados concretos do modelo e todos os caminhos válidos a partir do estado inicial $s_{0}$, por definição. Mostraremos que no processo de divisão de uma classe do CSZG 
nenhum estado é retirado, e as novas transições de estado satisfazem os caminhos válidos no modelo em $\mathrm{RPT}$.

O único ponto do algoritmo onde um estado de CSZG é modificado é quando a restrição é aplicada ao domínio temporal da classe identificada como não estável.

A restrição aplicada à classe instável é do tipo $\left\{t-t^{\prime}>c, t-t^{\prime} \leq c\right\}$ ou $\left\{t-t^{\prime}<c, t-t^{\prime} \geq c\right\}$ dependendo da condição que determinou a instabilidade. Sendo assim, a restrição representa uma reta no plano t, t' e portanto divide o conjunto das soluções do domínio temporal da classe instável em duas, ambas perfeitamente convexas.

Se uma das classes divididas ainda satisfaz à condição de instabilidade o processo se repete até a classe inicialmente identificada como instável tenha sido dividida em classes estáveis. O número de divisões é finito, uma vez que o número de transições habilitadas em cada classe também é finito.

Para cada classe de estado resultante do processo de divisão, uma nova transição é adicionada ao grafo, de forma que os caminhos na classe dividida são preservados. Sendo assim, nenhum estado concreto foi adicionado ou retirado da partição e os caminhos existentes na partição original continuam na partição após o processo de divisão.

\section{Teorema 15 (Preservação de propriedades de tempo ramificado pelo}

CSSG). Se o grafo das classes de estados de uma RPT $N$ é estável, então para cada classe de estado $C$ no grafo, qualquer estado concreto $s \in C$ e qualquer fórmula $C T L \phi, C \models \phi$ é equivalente a $s \models \phi$.

Prova Seja uma fórmula CTL $\phi$ definida indutivamente em (CLARKE; EMERSON; SISTLA, 1986). Existe uma decomposição da fórmula $\phi$ em sub-fórmulas CTL que por sua vez satisfaz à seguinte condição:

$$
(s \models \phi) \Leftrightarrow\left(s \models \theta_{1}\right) \wedge\left(s \models \theta_{2}\right) \wedge \ldots \wedge\left(s \models \theta_{j}\right)
$$

onde $\theta_{1}, \theta_{2}, \ldots, \theta_{j}$ são sub-fórmulas básicas da CTL e o resultado de cada uma delas é usado como uma proposição em alguma das sub-fórmulas seguintes.

Estas sub-fórmulas básicas podem ser divididas em dois grupos: as fórmulas de estado $(p, \neg p, p \wedge q, p \rightarrow q)$ e as fórmulas de caminho $(\exists[p U q], \forall[p U q], \exists \circ p, \forall \circ p)$.

Suponha que das $j$ sub-fórmulas, um número $i$ delas, $\theta_{1}, \theta_{2}, \ldots, \theta_{i}$ são fórmulas 
de estados. A validade destas fórmulas depende somente das proposições verdadeiras nesse estado. Pela definição de classe de estados podemos concluir que:

$$
\exists s \in S \exists C \in \Omega \text { tal que } \forall s \in C M(s)=M(C)
$$

Como $s$ e $C$ possuem a mesma marcação então as proposições verdadeiras em $s$ são também verdadeiras em $C$ e viceversa, portanto cumpre-se que:

$$
\begin{aligned}
\left(C \models \theta_{1}\right) & \Leftrightarrow \quad\left(s \models \theta_{1}\right) \\
\left(C \models \theta_{2}\right) & \Leftrightarrow \quad\left(s \models \theta_{2}\right) \\
& \vdots \\
\left(C \models \theta_{i}\right) & \Leftrightarrow \quad\left(s \models \theta_{i}\right)
\end{aligned}
$$

Suponha que as sub-fórmulas $\theta_{i+1}, \theta_{i+2}, \ldots, \theta_{j}$ sejam fórmulas de caminho. A validade destas fórmulas depende dos caminhos que começam em $s$.

Suponha então que $\lceil\lambda\rceil$ é o conjunto de todos os caminhos que começam em $s$ e $\left\lceil\lambda_{\theta}\right\rceil \subseteq\lceil\lambda\rceil$ o subconjunto destes caminhos que satisfazem $\theta$.

Pelo lema 14 sabemos que:

$$
\forall s^{\prime} \in \sigma, \sigma \in\lceil\lambda\rceil \quad \exists C^{\prime} \in \Omega \text { tal que } s^{\prime} \in C^{\prime}
$$

Como $C^{\prime}$ é estável cumpre-se que $\Upsilon\left(s^{\prime}\right)=\Upsilon\left(C^{\prime}\right)$ e pela definição de classe de estados $M\left(s^{\prime}\right)=M\left(C^{\prime}\right)$. Portanto existe no CSCG, a partir de $C$, os mesmos conjuntos de caminhos $\left\lceil\lambda_{\theta}\right\rceil$ e $\lceil\lambda\rceil$.

Sendo assim, pode-se afirmar que:

$$
\begin{aligned}
&\left(C \models \theta_{i+1}\right) \Leftrightarrow\left(s \models \theta_{i+1}\right) \\
&\left(C \models \theta_{i+2}\right) \Leftrightarrow\left(s \models \theta_{i+2}\right) \\
& \vdots \\
&\left(C \models \theta_{j}\right) \Leftrightarrow\left(s \models \theta_{j}\right) \\
&(C \models \phi) \Leftrightarrow\left(C \models \theta_{1}\right) \wedge\left(C \models \theta_{2}\right) \wedge \ldots \wedge\left(C \models \theta_{j}\right)
\end{aligned}
$$

Portanto pode-se concluir que $(C \models \phi) \Leftrightarrow(s \models \phi)$

A partição do espaço de estados obtida usando a abordagem das classes de estados com restrições satisfaz as condições (EE) e (EA). A condição (AE) não é satisfeita, mas a condição de estabilidade garante a preservação das propriedades 
de tempo ramificado.

Quanto ao cálculo dos incrementos de tempo entre domínios temporais sucessivos existem condições que garantem sua determinação correta. O cálculo destes incrementos de tempo é determinado usando a proposição 12.

A seguir é mostrado o sistema de inequações cuja solução dá o valor destes incrementos de tempo. Suponha que a transição de estados $C \stackrel{t_{f}}{\longrightarrow} C^{\prime}$ onde $C=$ $(M, D)$ e $C^{\prime}=\left(M^{\prime}, D^{\prime}\right)$ é uma transição admissível na partição $G=\left(\Omega, \stackrel{*}{\rightarrow}, C_{0}\right)$.

$$
\begin{aligned}
& \forall t \in\left\{\operatorname{enb}(M) \cap \operatorname{enb}\left(M^{\prime}\right)\right\} \quad \Delta_{\min } \geq D_{o t}^{\prime}-D_{o t} 0 \quad \Delta_{\max } \leq D_{t o}^{\prime}-D_{t o} \\
& t=t_{f} \quad \Delta_{\min } \geq E F T(t)-D_{o t} \quad \Delta_{\max } \leq L F T(t)-D_{t o} \\
& \forall t \in \operatorname{enb}(M) \quad \Delta_{\max } \leq L F T(t)-D_{t o} \\
& l\left(t_{f}\right)=\max \left(\Delta_{\min }\right), \quad u\left(t_{f}\right)=\min \left(\Delta_{\max }\right)
\end{aligned}
$$

O sistema de inequações é formado pelas inequações dos domínios sucessivos que compartilham transições habilitadas e pelos intervalos estáticos de disparo das transições habilitadas na classe que originou a mudança de estado. A solução do sistema $\left[l\left(t_{f}\right), u\left(t_{f}\right)\right]$ dá os incrementos de tempo mínimo e máximo, respectivamente, na transição de estado $C \stackrel{t_{f}}{\longrightarrow} C^{\prime}$.

Os valores dos domínios temporais foram obtidos através do algoritmo que computa o estado sucessor, e como no CSCG a condição (EA) é satisfeita, todos os estados concretos que pertencem à classe de estados $C$ - e satisfazem a restrição imposta pelo intervalo de disparo estático da transição envolvida no disparo têm um sucessor na classe de estados $C^{\prime}$. Sendo assim, a solução do sistema de inequações levará a valores corretos dos incrementos de tempo global se o grafo satisfaz a condição (EA).

Na determinação das classes de estados com restrições foi introduzido o valor $\epsilon$ para delimitar os estados concretos que estão na restrição e os que ficam de fora. Desta forma as relações binárias usadas nas restrições passam a ser apenas $\{<,>\}$ em vez de $\{\leq,<,>, \geq\}$. O valor de $\epsilon$ pode ser escolhido antes de executar o algoritmo da geração das classes de estados com restrições, e de modo geral se deve usar um valor bem menor que o menor valor de tempo usado nos intervalos das transições.

A tabela 3.5 mostra as classes de estados com restrições obtidas para o exemplo da figura 3.2 usando $\epsilon=0.01$. Considerando estes domínios temporais pode-se notar que todos os estados possíveis na RPT da figura 3.2 estão contemplados na partição das classes de estados com restrições mostrada na tabela 3.5. O grafo 


\begin{tabular}{|c|c|c|c|c|}
\hline Classe de est. & Marcação & Dom. temporal & Incremento & Transições \\
\hline class 0 & p1 p6 & $\begin{array}{l}0 \leq t 1 \leq 0 \\
0 \leq t 5 \leq 0\end{array}$ & {$[0,1]$} & $C 0 \stackrel{t 1[0,1]}{\longrightarrow} C 1$ \\
\hline class 1 & p2 p6 & $\begin{array}{l}0 \leq t 2 \leq 0 \\
0 \leq t 5 \leq 1\end{array}$ & {$[0,1]$} & $C 1 \stackrel{t 2[0,1]}{\longrightarrow} C 2$ \\
\hline class 2 & p3 p6 & $\begin{array}{l}0 \leq t 3 \leq 0 \\
0 \leq t 5 \leq 2\end{array}$ & $\begin{array}{c}{[2,1]} \\
{[0,-0.01]}\end{array}$ & $\begin{array}{l}C 2 \stackrel{t 3[0,1]}{\longrightarrow} C 3 \\
C 2 \stackrel{t 3[0,1]}{\longrightarrow} C 4\end{array}$ \\
\hline class 3 & $\mathrm{p} 4 \mathrm{p} 6$ & $\begin{array}{l}0 \leq t 4 \leq 0 \\
2 \leq t 5 \leq 3\end{array}$ & $\begin{array}{l}{[1,3]} \\
{[3,3]} \\
\end{array}$ & $\begin{array}{l}C 3 \stackrel{t 4[1,3]}{\longrightarrow} C 5 \\
C 3 \stackrel{t 5[2,3]}{\longrightarrow} C 8\end{array}$ \\
\hline class 4 & $\mathrm{p} 4 \mathrm{p} 6$ & $\begin{array}{c}0 \leq t 4 \leq 0 \\
0 \leq t 5 \leq 1.99\end{array}$ & {$[1,3]$} & $C 4 \stackrel{t 4[1,3]}{\longrightarrow} C 6$ \\
\hline class 5 & p5 p6 & $3 \leq t 5 \leq 6$ & {$[2,0]$} & $C 5 \stackrel{t 5[0,3]}{\longrightarrow} C 7$ \\
\hline class 6 & p5 p6 & $1 \leq t 5 \leq 4.99$ & {$[4,1.01]$} & $C 6 \stackrel{t 5[0.01,5]}{\longrightarrow} C 7$ \\
\hline class 7 & p5 p7 & & & \\
\hline class 8 & $\mathrm{p} 4 \mathrm{p} 7$ & $2 \leq t 4 \leq 3$ & {$[0,0]$} & $C 8 \stackrel{t 4[0,1]}{\longrightarrow} C 7$ \\
\hline
\end{tabular}

Tabela 3.5: Classes de estado com restrições para o exemplo da figura 3.2

das classes de estados com restrições que representa o comportamento temporal da RPT da figura 3.2 é mostrado na figura 3.8.

Como se pode ver, as classes $C 3$ e $C 4$ estão relacionadas à restrição $a$ da figura 3.7. A restrição $b$ não tem influencia nenhuma no comportamento ramificado e portanto não foi preciso dividir a classe $C 2$. Note-se que mesmo $C 2$ sendo nãoatômica (existem estados em $C 2$ que não tem sucessores em $C 3$ ) a estrutura da figura 3.8 satisfaz as mesmas fórmulas CTL que a estrutura ASCG das figuras 3.2 e 3.3 .

O tamanho do grafo na figura 3.8 é menor do que aquele obtido usando o algoritmo em (YONEDA; RYUBA, 1998) e também menor que o obtido usando o algoritmo em (BERTHOMIEU; VERNADAT, 2003). A restrição $C 3^{\prime}-C 0 \geq 2$ e $C 3^{\prime}-C 0<2$ foi aplicada diretamente no domínio da classe $C 3^{\prime}$ obtendo as classes $C 3$ e $C 4$.

\subsubsection{O grafo das transições temporizadas}

Para garantir a terminação de qualquer processo de verificação de modelos devese garantir que a estrutura sobre a qual a verificação será feita é finita. O seguinte teorema suporta esta garantia. 


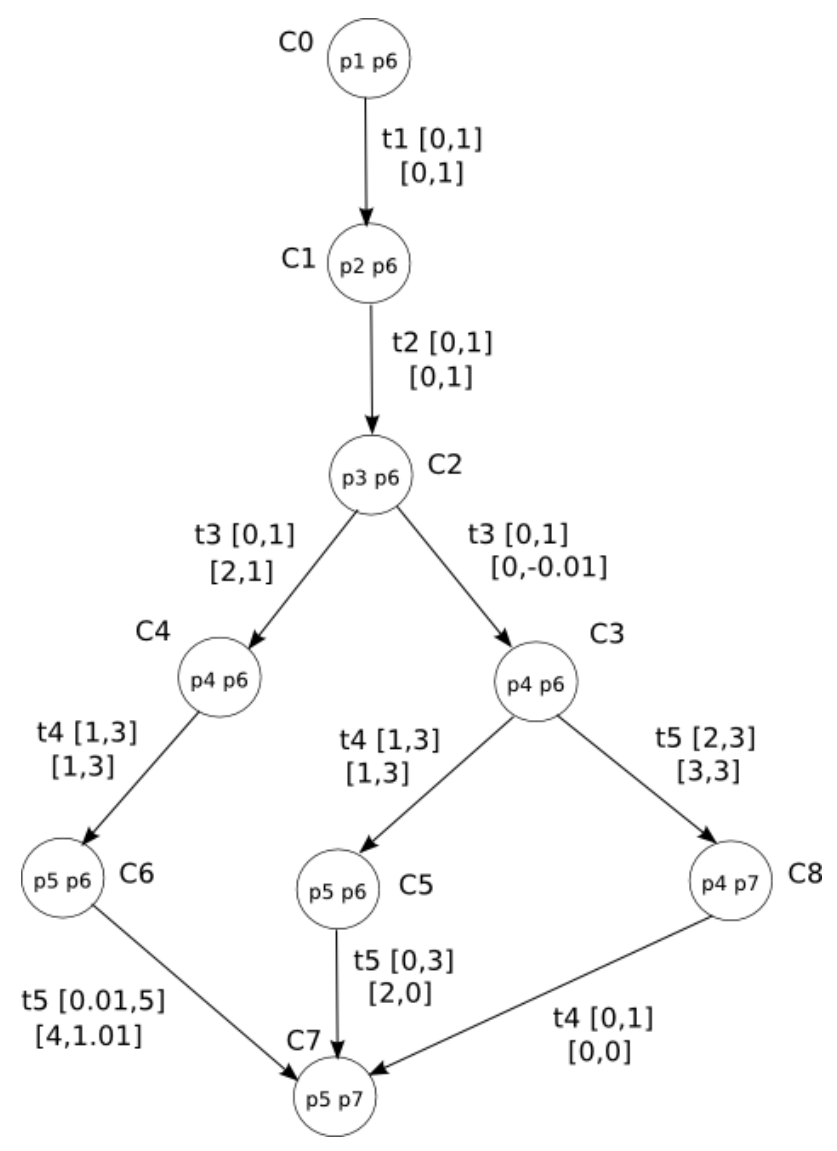

Figura 3.8: Grafo das classes de estados com restrições da RPT na figura 3.2

Teorema 16 (Finitude do CSCG). O CSCG é finito sse o CZSG é finito

Prova $\Rightarrow:$ Se o CSCG é finito, significa que o número de marcações atingíveis é também finito, portanto a RPT é limitada. Pelo teorema 4 em (HADJIDJ; BOUCHENEB, 2008) conclui-se que o CZSG também é finito .

$\Leftarrow$ : Como foi mostrado na prova do lema 14, o processo de divisão das classes de estados aplicando uma restrição gera um número finito de classes de estados para cada classe de estado não estável do CSZG. Sendo assim, se o CSZG é finito o CSCG será também finito.

Define-se a estrutura construída a partir da partição obtida usando as classes de estados com restrições ou CSSG como segue:

Definição 17 (Grafo das transições temporizadas). O grafo das transições temporizadas (GTT) é uma tupla $G=\left(S, \mu, s_{0}, A, E, I t\right)$, onde:

- S é um conjunto finito de nós; 
- $\mu: S \rightarrow P$ é a função de rotulação que define o conjunto de proposições atômicas que são verdadeiras em cada nó;

- $s_{0} \in S$ é o nó inicial;

- A é o conjunto de ações ou eventos;

- $E \subseteq S \times A \times S$ é o conjunto de arestas;

- It : $E \longrightarrow Q^{+} \times\left(Q^{+} \cup\{\infty\}\right) \times Q \times(Q \cup\{\infty\})$ é o intervalo de tempo associado a cada aresta do grafo que representa o tempo de disparo mínimo e o máximo que pode transcorrer até que o sistema mude de estado através da ocorrência do evento $a \in A$ e o incrementos de tempo global minimo e máximo associados à ocorrência do deste evento;

O intervalo It : $[\delta(a), l, u]$ associado a cada transição no GTT representa o intervalo de disparo da transição $a$ no estado de partida assim como o incremento de tempo global mínimo e máximo para o sistema mudar de estado. Esses valores de tempo no intervalo It estão associados à ocorrência de um evento específico.

O GTT é construído a partir da partição das classes de estados com restrições da seguinte forma:

1. Cada classe de estados $C \in \Omega$ é representada por um nó do GTT $s \in S$, estabelecendo uma relação biunívoca entre estes conjuntos.

2. A função de rotulação $\mu$ é definida relacionando a marcação de cada classe de estados com as proposições verdadeiras do nó correspondente do GTT.

3. O conjunto de eventos do GTT é o mesmo da RPT exceto pela a existência de alguma transição "morta" 5 .

4. As arestas do GTT são formadas pelas transições das classes de estado seguindo a relação entre os nós e as classes de estados determinadas no primeiro passo.

5. O intervalo ligado a cada aresta é o intervalo de tempo associado à transição disparada em cada caso.

Como o GTT é construído a partir do CSCG pode-se afirmar que o GTT é finito sse o CSCG é finito.

\footnotetext{
${ }^{5}$ São assim definidas as transições que não disparam em nenhuma das marcações atingidas a partir do estado inicial
} 


\subsubsection{A verificação da TCTL sobre o grafo das transições temporizadas}

Para verificar a lógica TCTL, precisamos de uma estrutura que preserve as propriedades de tempo ramificado, onde também possam ser computados os tempos mínimos e máximos para cada caminho no grafo. O algoritmo que computa as classes com restrições gera uma partição do espaço de estados a partir do qual o GTT é construído. Neste grafo, cada nó representa uma classe de estado e portanto temos o incremento de tempo mínimo e máximo para cada transição, o que é representado pelas arestas do GTT.

Sendo assim, suponha que a seguinte seqüência de transições, válida sobre o grafo $G$ :

$$
\omega=s_{i} \stackrel{a_{1},\left[l_{i+1}, u_{i+1}\right]}{\longrightarrow} s_{i+1} \stackrel{a_{2},\left[l_{i+2}, u_{i+2}\right]}{\longrightarrow} s_{i+2} \ldots s_{i+n-1} \stackrel{a_{j},\left[l_{i+n}, u_{i+n}\right]}{\longrightarrow} s_{i+n}
$$

onde cada par $\left[l_{x}, u_{x}\right]$ com $x=i+1 \ldots n$ representa o incremento de tempo mínimo e máximo associado a cada disparo.

Os incrementos de tempo mínimos e máximos são computados através dos domínios temporais das classes de estados de tal forma que estes incrementos levam em consideração o tempo de disparo da transição que antecede este estado. Portanto, nem sempre o valor dos incrementos de tempo mínimo e máximo para o estado onde começa o caminho $\omega$ correspondem aos valores reais.

Se computássemos o tempo mínimo a partir de um estado onde todas as transições são recém habilitadas, como é o caso do estado inicial, o tempo mínimo corresponderia à somatória do incremento dos tempos mínimos das transições disparadas até atingir o estado desejado. Contudo, é necessário também poder calcular tempos mínimos e máximos em caminhos que não começam no estado inicial.

A solução para este problema é simples. Substituímos o incremento de tempo mínimo e máximo do estado inicial do caminho pelo intervalo de disparo associado à transição que foi disparada, e então teremos os tempos reais mínimos e máximos de transição ao próximo estado do caminho, sem considerar tempos de disparo anteriores a este estado inicial.

No exemplo do caminho $\omega$, o tempo mínimo transcorrido entre $s_{i}$ e $s_{i+n}$ corresponde à somatória do incremento dos tempos mínimos nas transições que pertencem ao caminho $\omega, a_{2}, \ldots, a_{j}{ }^{6}$, mais o tempo mínimo de disparo da transição

\footnotetext{
${ }^{6}$ Note que podem existir transições repetidas no caminho $\omega$
} 
$a_{1}$ em $s_{i}$.

Igualmente, o tempo máximo entre $s_{i}$ e $s_{i+n}$ depende da somatória dos incrementos dos tempos máximos nas transições que pertencem ao caminho $\omega$ e do tempo máximo de disparo da primeira transição no caminho.

Assim, os tempos mínimo e máximo entre $s_{i}$ e $s_{i+n}$ no caminho $\omega$ seriam calculados como:

$$
\begin{aligned}
& t_{\omega \min }=\sum_{x=i+2}^{i+n} l_{x}+\downarrow \delta_{s_{i}}\left(a_{1}\right) \\
& t_{\omega \max }=\sum_{x=i+2}^{i+n} u_{x}+\uparrow \delta_{s_{i}}\left(a_{1}\right)
\end{aligned}
$$

Dado que o GTT preserva as propriedades de tempo ramificado e os tempos mínimos e máximos podem ser calculados para todos os caminhos em $G$, podemos chegar à seguinte definição:

Definição 18 (estrutura TCTL). O grafo das transições temporizadas é uma estrutura TCTL.

Ao contrário de outras abordagens que usam as RPT na verificação de modelos, não temos a limitação de usar só RPT seguras (1-limitadas). Sendo assim podemos encontrar marcações de classes de estados como por exemplo:

$$
M_{4}=(p 1 \quad p 2 * 3 \quad p 5 \quad p 7 * 2)
$$

E como no GTT as marcações da RPT determinam o conjunto de proposições atômicas verdadeiras em cada estado a mesma é convertida da seguinte forma:

$$
\mu_{4}=\left(\begin{array}{lllllll}
p 1 & p 2 * 3 & p 2 * 2 & p 2 & p 5 & p 7 * 2 & p 7
\end{array}\right)
$$

onde cada um destes elementos é uma proposição verdadeira no estado $s_{4}=$ $\left(M_{4}, I_{4}\right)$.

\subsection{O verificador de modelos}

O problema da verificação de modelos neste trabalho reduz-se a determinar se $(G, s) \models \phi$ é verdadeira para uma fórmula $\phi$ expressa usando TCTL, uma estrutura $G$ definida como um GTT, e um estado $s \in S$. 
Na seção 3.3 mostramos como o GTT é construído a partir das classes de estados, as quais representam o conjunto de estados do sistema que de fato precisamos verificar. O primeiro passo para garantir que os resultados obtidos pelo verificador de modelos é provar que estes resultados, válidos para as classes de estados, são também válidos para os estados que formam estas classes de estados.

Teorema 19 (Decidabilidade da TCTL sobre o GTT). Dada uma fórmula TCTL $\phi$ e um GTT construído a partir das classes de estados com restrições $C \in \Omega$, para todo estado $s \in C, C \models \phi$ é equivalente a $s \models \phi$.

Prova Seja uma fórmula TCTL $\phi$ definida indutivamente segundo a definição 1. Portanto, existe uma decomposição da fórmula $\phi$ em sub-fórmulas TCTL que satisfazem à seguinte condição:

$$
(s \models \phi) \Leftrightarrow\left(s \models \theta_{1}\right) \wedge\left(s \models \theta_{2}\right) \wedge \ldots \wedge\left(s \models \theta_{j}\right)
$$

onde $\theta_{1}, \theta_{2}, \ldots, \theta_{j}$ são sub-fórmulas básicas da TCTL e o resultado de cada uma delas é usado como uma proposição em alguma das sub-fórmulas sub seguintes.

As sub-fórmulas básicas podem ser divididas em dois grupos: as fórmulas de estado $(p, \neg p, p \wedge q, p \rightarrow q)$ e as fórmulas de caminho $\left(\exists\left[p U_{\sim_{c}} q\right], \forall\left[p U_{\sim_{c}} q\right], p-q \sim c\right)$.

Como a validade das fórmulas de estados não depende do tempo o argumento é o mesmo que o utilizado na prova do teorema 15. Portanto cumpre-se que:

$$
\begin{aligned}
\left(C \models \theta_{1}\right) & \Leftrightarrow\left(s \models \theta_{1}\right) \\
\left(C \models \theta_{2}\right) & \Leftrightarrow \quad\left(s \models \theta_{2}\right) \\
& \vdots \\
\left(C \models \theta_{i}\right) & \Leftrightarrow \quad\left(s \models \theta_{i}\right)
\end{aligned}
$$

Suponha que as sub-fórmulas $\theta_{i+1}, \theta_{i+2}, \ldots, \theta_{j}$ sejam fórmulas de caminho. A validade destas vai depender dos caminhos que começam em $s$ e da satisfatibilidade, nesses caminhos, da restrição de tempo em cada uma das fórmulas.

Os seguintes argumentos são válidos para todas as fórmulas de caminho, se pode eleger uma sub-fórmulas genérica $\theta$.

Suponha então que $\left\lceil\lambda_{\theta}\right\rceil$ é o conjunto de todos os caminhos que começam em $s$ nos quais $\theta$ é válida. Seja $\sigma \in\left\lceil\lambda_{\theta}\right\rceil$ definida como:

$$
\sigma=s \stackrel{a_{1}, d_{1}}{\longrightarrow} s_{1} \stackrel{a_{2}, d_{2}}{\longrightarrow} \ldots \stackrel{a_{n-1}, d_{n-1}}{\longrightarrow} s_{n-1} \stackrel{a_{n}, d_{n}}{\longrightarrow} s_{n}
$$


onde o caminho $s \rightarrow s_{1} \rightarrow \ldots \rightarrow s_{n}$ satisfaz a semântica CTL de $\theta$ e o tempo $t=\sum_{i=1}^{n} d_{i}$ satisfaz a restrição temporal em $\theta$.

Como o GTT foi construído a partir do CSCG temos que, para cada caminho $\sigma \in\left\lceil\lambda_{\theta}\right\rceil$ vai existir um caminho no GTT começando em $C$ :

$$
\sigma_{\Omega}=C \stackrel{a_{1},\left[l_{1}, u_{1}\right]}{\longrightarrow} C_{1} \ldots C_{n-1} \stackrel{a_{j},\left[l_{n}, u_{n}\right]}{\longrightarrow} C_{n}
$$

tal que $s \in C, s_{1} \in C_{1}, \ldots, s_{n} \in C_{n}$

Com relação ao tempo, existem diferentes casos dependendo do quantificador de caminhos e do tipo de restrição temporal na fórmula $\theta$.

Analisemos o caso de $\exists\left[p U_{<c} q\right]$.

No caminho $\sigma$ temos que $t=\sum_{i=1}^{n} d_{i}$ e como $s \models \theta$ então temos que $t<c$. Como $\sigma$ é um caminho válido em $S$, pelo algoritmo de construção do GTT existe $\sigma_{\Omega}$ onde se cumpre que:

$$
\Delta_{\sigma_{\Omega}} \min \leq t \leq \Delta_{\sigma_{\Omega}} \max
$$

Como $t<c$ tem-se que $\Delta_{\sigma_{\Omega}}$ min $<c$, o que garante que $C \models \exists\left[p U_{<c} q\right]$, portanto $\left(s \models \exists\left[p U_{<c} q\right]\right) \Rightarrow\left(C \models \exists\left[p U_{<c} q\right]\right)$. O restante dos casos é facilmente dedutível a partir deste, e portanto se pode afirmar o seguinte:

$$
(s \models \theta) \Rightarrow(C \models \theta)
$$

Suponha agora que $C \models \theta$ é verdadeira. Sem perda de generalidade vamos referirmo-nos a uma fórmula em específico, lembrando que o resto dos casos podem ser dedutíveis a partir deste.

Tem-se que $C \models \forall\left[p U_{<c} q\right]$. Isto implica que todos os caminhos no GTT que começam em $C$ satisfazem $[p U q]$ em um tempo inferior a $c$ unidades de tempo.

Pelo algoritmo de construção do GTT e sabendo que as classes de estados com restrições satisfazem as propriedades (EE) e (C) podemos dizer que todos os caminhos de $S$ que começam em $s \in C$ estão representados em $\Omega$ e correspondem a todos os caminhos que começam em $C$.

Como $C=\forall\left[p U_{<c} q\right]$ é verdadeiro, então todas as classes de estados $C_{x} \in \Omega$ onde a formula $p U q$ é satisfeita têm $\Delta_{\sigma_{C_{x}}} \max <c$. Denota-se por $\sigma_{C_{x}}$ todas os caminhos a partir de $C$ que terminam em $C_{x}$.

Portanto, todos os caminhos em $S$ que começam em $s \in C$ e satisfazem 
$p U q$ terminam em estados $s^{\prime} \in C_{x}$ com tempos $t \leq \Delta_{\sigma_{C_{x}}}$ max, portanto se pode afirmar que:

$$
\left(C \models \forall\left[p U_{<c} q\right]\right) \Rightarrow\left(s \models \forall\left[p U_{<c} q\right]\right)
$$

A partir deste resultado pode-se deduzir que:

$$
(C \models \theta) \Rightarrow(s \models \theta)
$$

Levando em consideração 3.13 e 3.14 tem-se que:

$$
(C \models \theta) \Leftrightarrow(s \models \theta)
$$

Então poderemos chegar a seguinte conclusão:

$$
\begin{aligned}
\left(C \models \theta_{i+1}\right) & \Leftrightarrow \quad\left(s \models \theta_{i+1}\right) \\
\left(C \models \theta_{i+2}\right) & \Leftrightarrow \quad\left(s=\theta_{i+2}\right) \\
& \vdots \\
\left(C \models \theta_{j}\right) & \Leftrightarrow \quad\left(s \models \theta_{j}\right)
\end{aligned}
$$

Como também se cumpre que:

$$
(C \models \phi) \Leftrightarrow\left(C \models \theta_{1}\right) \wedge\left(C \models \theta_{2}\right) \wedge \ldots \wedge\left(C \models \theta_{j}\right)
$$

pode-se concluir que $(C \models \phi) \Leftrightarrow(s \models \phi)$

Do teorema 19 pode-se concluir que a lógica TCTL é decidível sobre o GTT construído usando as classes de estados com restrições. Este garante que o resultado obtido do verificador para cada fórmula TCTL é válido para os estados que representam o comportamento modelado usando as RPT.

O verificador de modelos responde sim ou não dada uma fórmula TCTL e uma estrutura especificada pelo GTT. Para que este procedimento tenha terminação garantida a estrutura deve ter um número finito de estados o que é garantido pelo uso da abordagem das classes de estados para a construção do GTT.

Contudo, o problema da verificação de modelos pode ser formulado de duas formas diferentes: global e local. O problema da verificação global determina a validade da fórmula em todos os estados da estrutura, assim, se a fórmula é válida para pelo menos um desses estados o verificador responde sim. O problema de verificação local determina a validade da fórmula em um determinado estado, o qual deve ser especificado conjuntamente com a fórmula e a estrutura. 
Usualmente, o problema da verificação local é menos complexo que o global uma vez que o mesmo é um subconjunto do problema de verificação global. Além disso, no caso da verificação local, contraexemplos ou justificativas podem ser fornecidos para mostrar porque uma determinada propriedade falhou.

\subsubsection{Preparando o processo de verificação}

A seguir apresentaremos algumas definições úteis no tratamento das fórmulas TCTL.

Definição 20 (Conjunto das sub-fórmulas). Seja $\phi$ uma fórmula TCTL, o conjunto de sub-fórmulas sub( $\phi)$ de $\phi$ é definido recursivamente como segue:

1. se $\phi$ é uma proposição atômica $p \in P$, então $\operatorname{sub}(\phi)=\{\phi\}$.

2. se $\phi$ é da forma $\neg \phi_{1}$, então $\operatorname{sub}(\phi)=\{\phi\} \cup \operatorname{sub}\left(\phi_{1}\right)$.

3. se $\phi$ é da forma $\phi_{1} \wedge \phi_{2}, \phi_{1} \rightarrow \phi_{2}, \exists\left[\phi_{1} U_{\sim_{c}} \phi_{2}\right], \forall\left[\phi_{1} U_{\sim_{c}} \phi_{2}\right]$, ou $\phi_{1}-\phi_{2} \sim c$ então $\operatorname{sub}(\phi)=\{\phi\} \cup \operatorname{sub}\left(\phi_{1}\right) \cup \operatorname{sub}\left(\phi_{2}\right)$.

Definição 21 (Conjunto das sub-fórmulas próprias). Seja $\phi$ uma fórmula TCTL, o conjunto de sub-fórmulas próprias psub $(\phi)$ de $\phi$ é definido por:

$$
\operatorname{psub}(\phi)=\operatorname{sub}(\phi) \backslash\{\phi\}
$$

Definição 22 (Comprimento da fórmula). Seja $\phi$ uma fórmula TCTL, o comprimento da fórmula $\phi$ é definido pela cardinalidade do conjunto sub( $\phi)$, ou seja:

$$
\text { length }(\phi)=|\operatorname{sub}(\phi)|
$$

A decomposição das fórmulas deve ser feita até que seja gerado o conjunto das sub-fórmulas próprias, o qual deve ser ordenado seguindo o seguinte ordem de precedência:

1. todas as fórmulas de estado, começando pelas de menor comprimento,

2. todas as fórmulas de caminho, começando pelas mais internas. 
Por exemplo, suponha que se deseja verificar a fórmula TCTL $\phi=\forall\left[p U_{\sim c} \exists[q U \neg p]\right]$. O conjunto das sub-fórmulas de $\phi$ seria:

$$
\left\{p, q, \neg p, \exists[q U \neg p], \forall\left[p U_{\sim c} \exists[q U \neg p]\right]\right\}
$$

O próximo passo é a representação da estrutura através da relação de sucessão dos estados e dos tempos em que as transições de estado ocorrem. Isto é feito usando os seguintes elementos:

- Um operador que retorna o conjunto dos estados sucessores de um determinado estado. Denominaremos este operador de $R$.

- Um operador que retorna o tempo mínimo no disparo da transição entre dois estados vizinhos. Por exemplo, o tempo mínimo de disparo da transição entre os estados $s$ e $s^{\prime}$ é denominado $\operatorname{Tmin}\left(s, s^{\prime}\right)$.

- Um operador que retorna o tempo máximo no disparo da transição entre dois estados vizinhos. Neste caso o tempo máximo na transição entre os estados $s$ e $s^{\prime}$ é denominado como $T \max \left(s, s^{\prime}\right)$.

- Um operador que devolve o incremento de tempo global mínimo da transição entre os estados $s$ e $s^{\prime}$ é denominado $T \operatorname{Min} G\left(s, s^{\prime}\right)$.

- Um operador que devolve o incremento de tempo global máximo da transição entre os estados $s$ e $s^{\prime}$ é denominado $T \operatorname{Max} G\left(s, s^{\prime}\right)$.

O operador $R(s)$ é definido formalmente da seguinte forma:

$$
R: S \rightarrow S^{n}, \forall s \in S, R(s)=s^{\bullet}
$$

Por exemplo, na figura 3.8, $R\left(s_{1}\right)=\left\{s_{2}, s_{3}\right\}$.

Os operadores $\operatorname{Tmin}\left(s_{1}, s_{2}\right)$ e $\operatorname{Tmax}\left(s_{1}, s_{2}\right)$ retornam respectivamente os tempos mínimo e máximo da ocorrência da transição que leva do estado $s_{1}$ ao estado $s_{2}$. Formalmente: Tmin $: S \times S \rightarrow Q^{+}$e Tmax $: S \times S \rightarrow Q^{+}$.

$\operatorname{Tmin}\left(s_{1}, s_{2}\right)=\left\{\begin{aligned} \downarrow \delta(a) & \text { sse } \exists\left(s_{1}, a, s_{2}\right) \in E \wedge a \in \operatorname{clk} \wedge \operatorname{It}\left(s_{1}, a, s_{2}\right)=[\delta(a), l, u] \\ 0 & \text { nos casos restantes }\end{aligned}\right.$ $\operatorname{Tmax}\left(s_{1}, s_{2}\right)=\left\{\begin{aligned} \uparrow \delta(a) & \text { sse } \exists\left(s_{1}, a, s_{2}\right) \in E \wedge a \in \operatorname{clk} \wedge \operatorname{It}\left(s_{1}, a, s_{2}\right)=[\delta(a), l, u] \\ 0 & \text { nos casos restantes }\end{aligned}\right.$

Os operadores $\operatorname{TMin} G\left(s_{1}, s_{2}\right)$ e $\operatorname{TMax} G\left(s_{1}, s_{2}\right)$ retornam respectivamente os incrementos de tempo global mínimo e máximo da ocorrência da transição 
que leva do estado $s_{1}$ ao estado $s_{2}$. Formalmente: TMinG $: S \times S \rightarrow Q^{+}$e $T M a x G: S \times S \rightarrow Q$.

$\operatorname{TMinG}\left(s_{1}, s_{2}\right)= \begin{cases}l & \text { sse } \exists\left(s_{1}, a, s_{2}\right) \in E \wedge a \in c l k \wedge I t\left(s_{1}, a, s_{2}\right)=[\delta(a), l, u] \\ 0 & \text { nos casos restantes }\end{cases}$

$\operatorname{TMax} G\left(s_{1}, s_{2}\right)= \begin{cases}u & \text { sse } \exists\left(s_{1}, a, s_{2}\right) \in E \wedge a \in \operatorname{clk} \wedge \operatorname{It}\left(s_{1}, a, s_{2}\right)=[\delta(a), l, u] \\ 0 & \text { nos casos restantes }\end{cases}$

Na figura 3.8, $\operatorname{Tmin}\left(C_{2}, C_{4}\right)=0, \operatorname{Tmax}\left(C_{2}, C_{4}\right)=1, \operatorname{Tmin} G\left(C_{2}, C_{4}\right)=2$ e $\operatorname{Tmax} G\left(C_{2}, C_{4}\right)=1$. Note que se a transição através da qual acontece a mudança de estado não pertence ao relógio $c l k$ definido para a fórmula que está sendo verificada $\operatorname{Tmin}=\operatorname{Tmax}=\operatorname{Tmin} G=\operatorname{Tmax} G=0$ pois estas transições não contribuem para o tempo de fórmula.

Para qualquer um dos problemas de verificação citados aqui a fórmula deve ser decomposta e as sub-fórmulas devem ser colocadas em uma pilha de forma que sejam puxadas primeiro as sub-fórmulas de menor comprimento. Um procedimento como este é o primeiro estágio de qualquer verificador de modelos.

\subsubsection{A verificação de modelos de forma global}

Depois que a fórmula a ser verificada é decomposta em sub-fórmula e empilhada em uma variável tipo pilha, o algoritmo de verificação global deve determinar a validade da formula em cada um dos estados do GTT. Em seguida checamos se a fórmula a ser verificada é válida em algum dos estados. O verificador responderá sim, ou não dependendo do resultado. O seguinte algoritmo rotula os estados com as respectivas sub-fórmulas válidas.

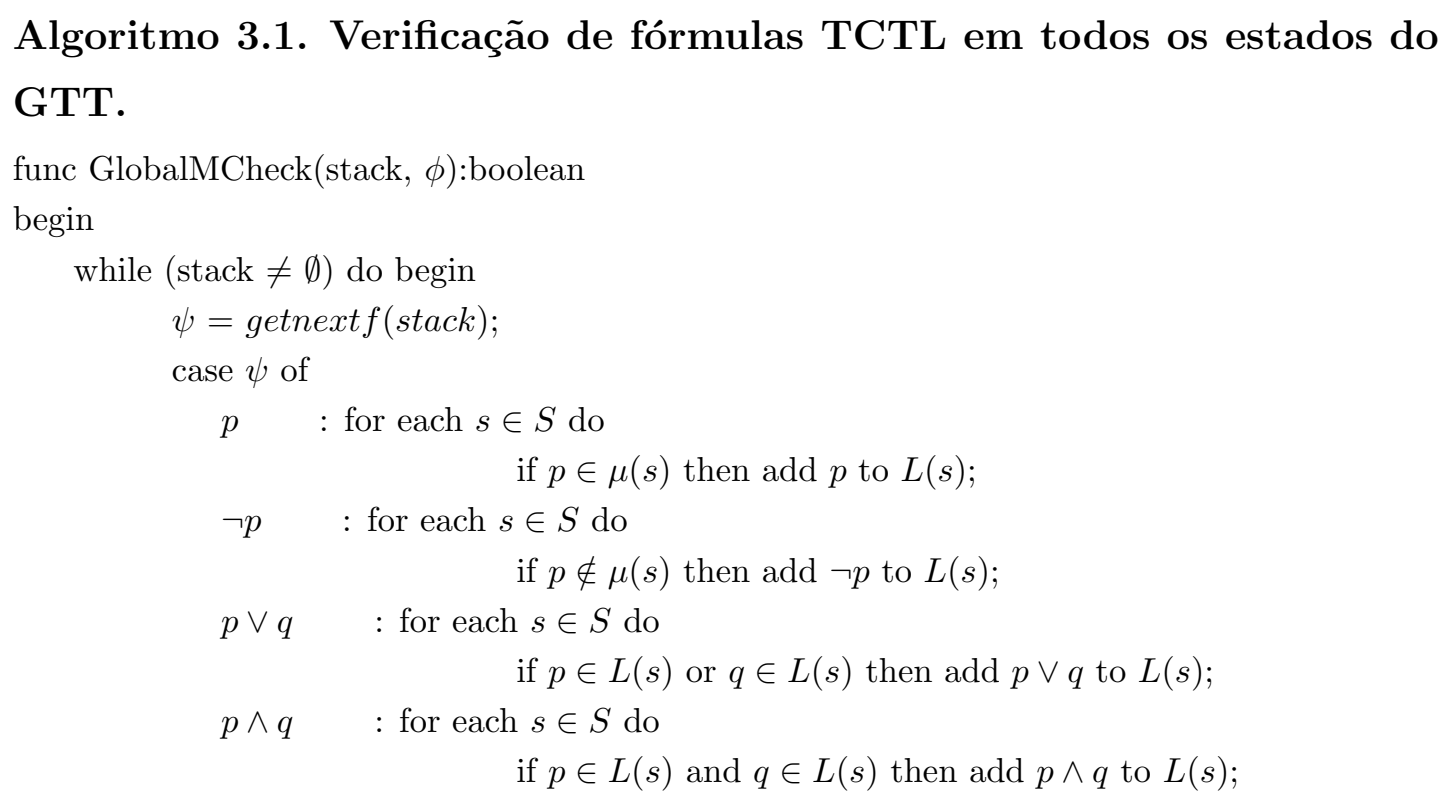




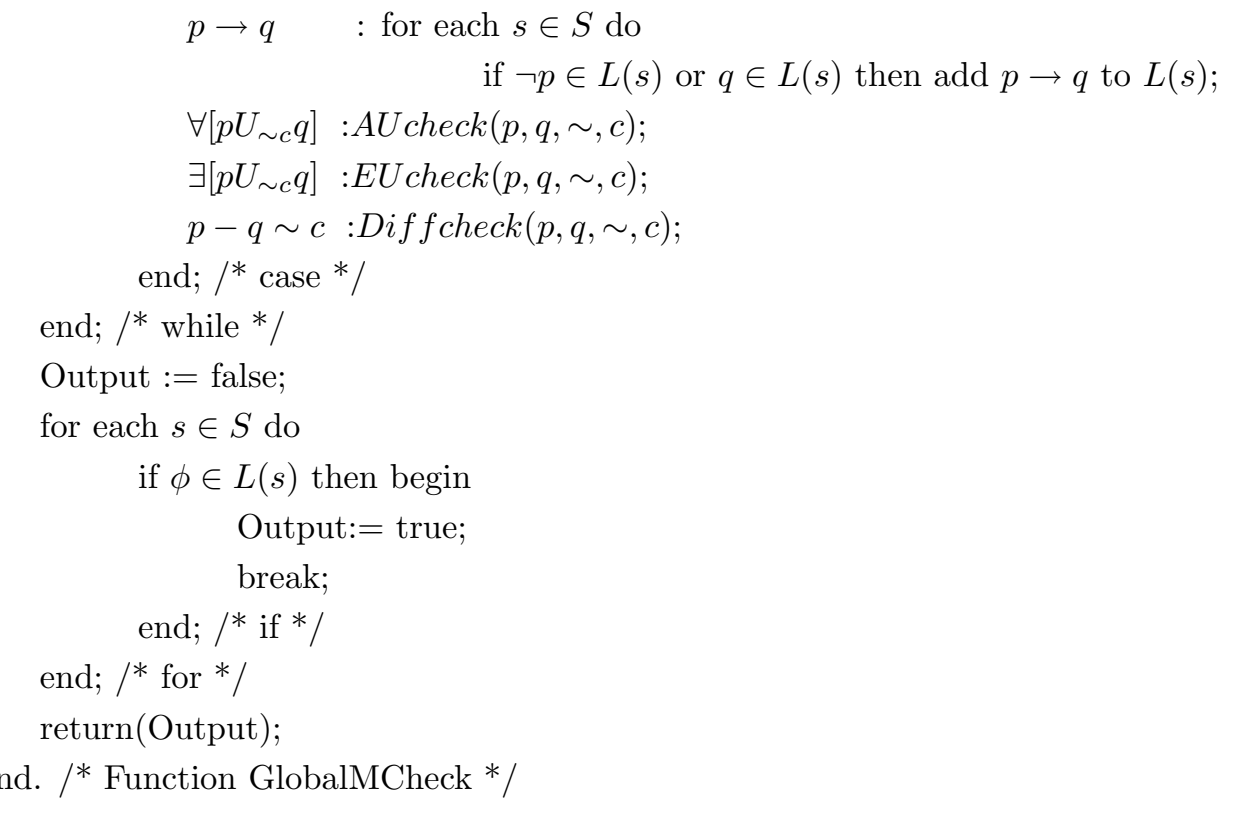

As subrotinas EUCheck e $A U C h e c k$ são as responsáveis pela determinação da validade das sub-fórmulas $\exists\left[p U_{\sim_{c}} q\right]$ e $\forall\left[p U_{\sim_{c}} q\right]$ respectivamente. A subrotina Diffcheck determina a validade das sub-fórmulas do tipo $p-q \sim c$.

Os algoritmos que verificam a validade de cada tipo de sub-fórmula usam duas estruturas de dados durante a execução: A primeira delas é um array de bits para marcar os estados que foram visitados pelo algoritmo. A segunda estrutura armazena os tempos máximo e mínimo para atingir os estados visitados bem como os tempos mínimo e máximo para atingir estados onde a fórmula $q$ foi avaliada positivamente. Estas estruturas são usadas para evitar visitar um estado mais de uma vez, e por conseguinte, para garantir a terminação da subrotina de forma eficiente.

A determinação da validade de sub-fórmulas de estados é simples e é feita de forma direta como mostrado no algoritmo 3.1. Já a determinação da validade das sub-fórmulas de caminhos é mais complexa e será discutida detalhadamente nas seções seguintes.

\subsubsection{Verificando as fórmulas UNTIL com quantificador universal}

Os verificadores em geral usam relações de equivalência para determinar de forma algorítmica o conjunto de estados que satisfazem determinada fórmula. Nos verificadores da lógica CTL a seguinte equivalência é usada para determinar os estados que satisfazem as fórmulas UNTIL com quantificador universal:

$$
\forall[\phi U \varphi] \equiv \varphi \vee(\phi \wedge \forall \circ(\forall[\phi U \varphi]))
$$

onde o é o operador "próximo estado" usado na CTL. 
Sejam $P$ e $Q$ os respectivos conjuntos de estados que satisfazem às fórmulas $\phi$ e $\varphi$. O conjunto de estados que satisfazem à fórmula $\forall \phi U \varphi$ é o limite de convergência da seqüencia de conjuntos $\left(A_{j}\right)$ definida da seguinte forma:

$$
\begin{aligned}
A_{0} & =Q \\
A_{k+1} & =A_{k} \cup\left\{s \mid(s \in P) \wedge\left(\forall s^{\prime} \mid\left(s,-, s^{\prime}\right) \in E\right) \wedge\left(s^{\prime} \in A_{k}\right\}\right.
\end{aligned}
$$

Apesar da TCTL não incluir o operador o, uma vez construído o GTT, é possível determinar o próximo estado e o tempo decorrido para executar uma transição no melhor ou pior caso. Levando isso em consideração, o conjunto de estados que satisfaz $\forall\left[p U_{\sim c} q\right]$ pode ser computado de forma similar à usada na CTL.

Seja $t_{r}$ o tempo transcorrido para atingir o estado $s$ a partir de um estado predecessor e $t_{q}$ o tempo transcorrido para atingir o estado onde $q \wedge \forall\left[p U_{\sim_{c}} q\right]$ é verdadeira. A fórmula $\forall\left[p U_{\sim_{c}} q\right]$ é válida em um estado $s \in S$ se e somente se:

$$
\underbrace{q \in L(s)}_{A} \vee \underbrace{\left(p \in L(s) \wedge\left(\forall s^{\prime} \in R(s) \mid \forall\left[p U_{\sim_{c}} q\right] \in L\left(s^{\prime}\right) \wedge\left(t_{r}+t_{q} \sim c\right)\right)\right)}_{B}
$$

lembrando que $\sim$ substitui um dos símbolos $<, \leq,=, \geq$, ou $>$. Note que a fórmula $\forall\left[p U_{<0} q\right]$ carece de sentido.

Suponha que estamos determinando os estados que satisfazem a fórmula $\forall\left[p U_{<c} q\right]$. Neste caso, o termo A é satisfeito em todos os estados onde $q$ é verdadeiro. O termo B é satisfeito em todos os estados onde $p$ é verdadeiro, todos os sucessores foram rotulados com a fórmula e todos esses sucessores são atingíveis em um tempo que satisfaz a restrição temporal. Dada a possibilidade de calcular o tempo máximo e mínimo através de uma seqüência de transições, este deve ser comparado com a constante $c$ e assim garantir que a restrição de tempo foi satisfeita.

Basta que um destes termos seja satisfeito para que a fórmula seja declarada válida nesse estado. Assim como em (CLARKE; EMERSON; SISTLA, 1986) e (HELJANKO, 1997) a idéia base é fazer uma busca em profundidade na estrutura procurando estados nos quais a condição 3.16 é satisfeita. O algoritmo 3.2 se encarrega de rotular os estados que satisfazem a fórmula $\forall\left[p U_{<c} q\right]$. A condição para que este algoritmo funcione corretamente é que as sub-fórmulas $p$ e $q$ estejam corretamente rotuladas em todos os estados da estrutura.

\section{Algoritmo 3.2. Subrotina AUcheck.}

func $\operatorname{AUcheck}(p, q, \sim, c)$

begin

for each $s \in S$ do begin 
state[s].visited $=$ false;

state[s].tmin $=0$;

state $[\mathrm{s}] \cdot \operatorname{tmax}=0$

state[s].qmin $=\infty$;

state[s].qmax $=0$;

end; $/ *$ for $* /$

List $=\left\{s_{0}\right\}$

while List $\neq \emptyset$ do begin

$\mathrm{s}=\mathrm{L} \mid$ List;

if $\neg$ state[s].visited then $\operatorname{DFS}(p, q, \sim, c$, state $[s] . t m i n$, state $[s] . t m a x, s$, out $, 0,0)$;

end; /* while */

end. /* function AUcheck */

A função $A U C h e c k$ inicializa as estruturas de dados que serão usadas na busca de estados que satisfazem a fórmula $\forall\left[p U_{\sim_{c}} q\right]$. Esta busca sempre começa no estado inicial do GTT. Assim, a subrotina DFS é chamada de forma recursiva em AUCheck até que todos os estados tenham sido visitados. O algoritmo 3.3 descreve o funcionamento desta subrotina.

Algoritmo 3.3. Busca em profundidade para o operador UNTIL com quantificador universal.

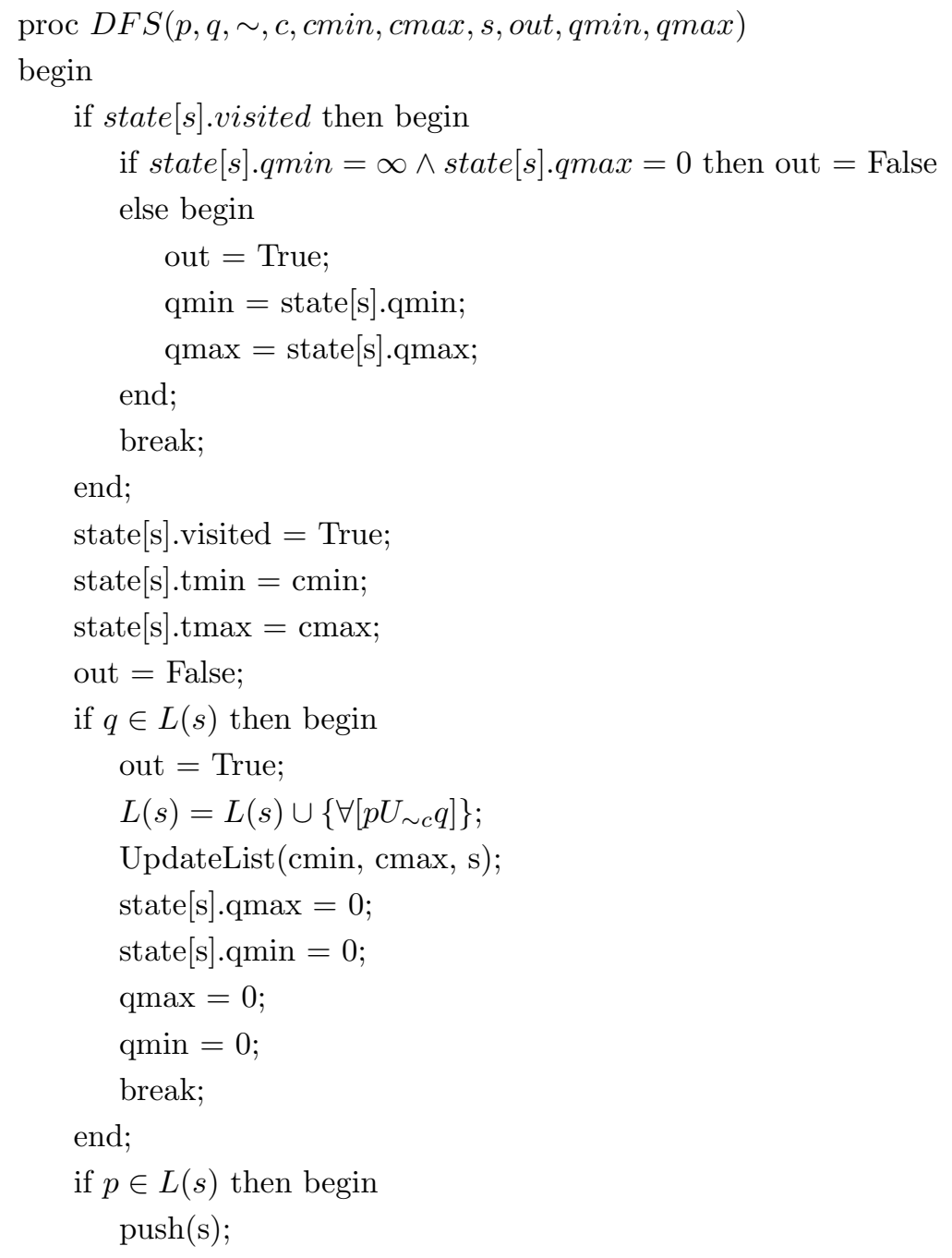




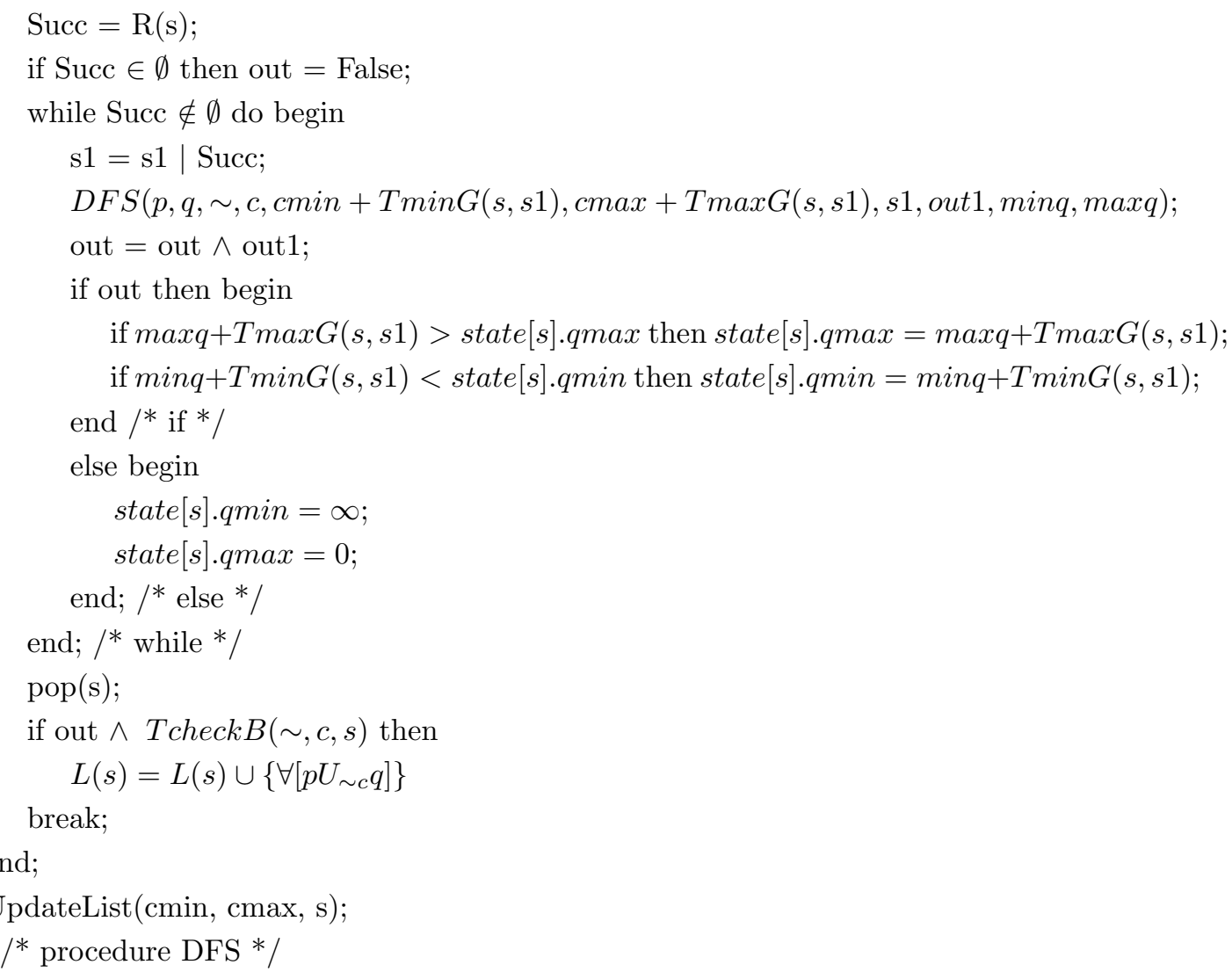

A busca em profundidade é sempre iniciada em um estado presente na variável List (inicialmente contendo só o estado inicial) o qual é removido imediatamente da lista. Sempre que a fórmula $p \wedge \neg q$ for verdadeira em um estado, este será colocado em uma variável tipo pilha e todos os sucessores deste estado serão processados chamando o próprio DFS de forma recursiva.

Cada vez que a fórmula $\neg p \wedge \neg q$ é encontrada, a variável List é atualizada com os sucessores do estado, desde que estes não estejam em List ou tenham sido previamente visitados. A busca é então abortada, e todos os estados na pilha são removidos.

No caso de $q$ ser verdadeira a fórmula $\forall\left[p U_{\sim_{c}} q\right]$ é declarada verdadeira e a variável List é atualizada com os sucessores deste estado desde que estes não estejam em List ou tenham sido previamente visitados. A partir deste ponto, os estados na pilha vão sendo retirados um a um, as variáveis que guardam o tempo transcorrido para atingir um estado onde $q$ é verdadeira são atualizadas e, somado ao tempo transcorrido para atingir o estado que estava na pilha e comparado com a restrição da fórmula. Se esta condição é satisfeita, o que é feito na subrotina $T C h e c k B$, o estado também é rotulado com a fórmula $\forall\left[p U_{\sim_{c}} q\right]$.

\section{Algoritmo 3.4. Subrotina TcheckB usada pela subrotina DFS.}

func TcheckB $(\sim, c$, pstate $)$ : boolean;

begin 


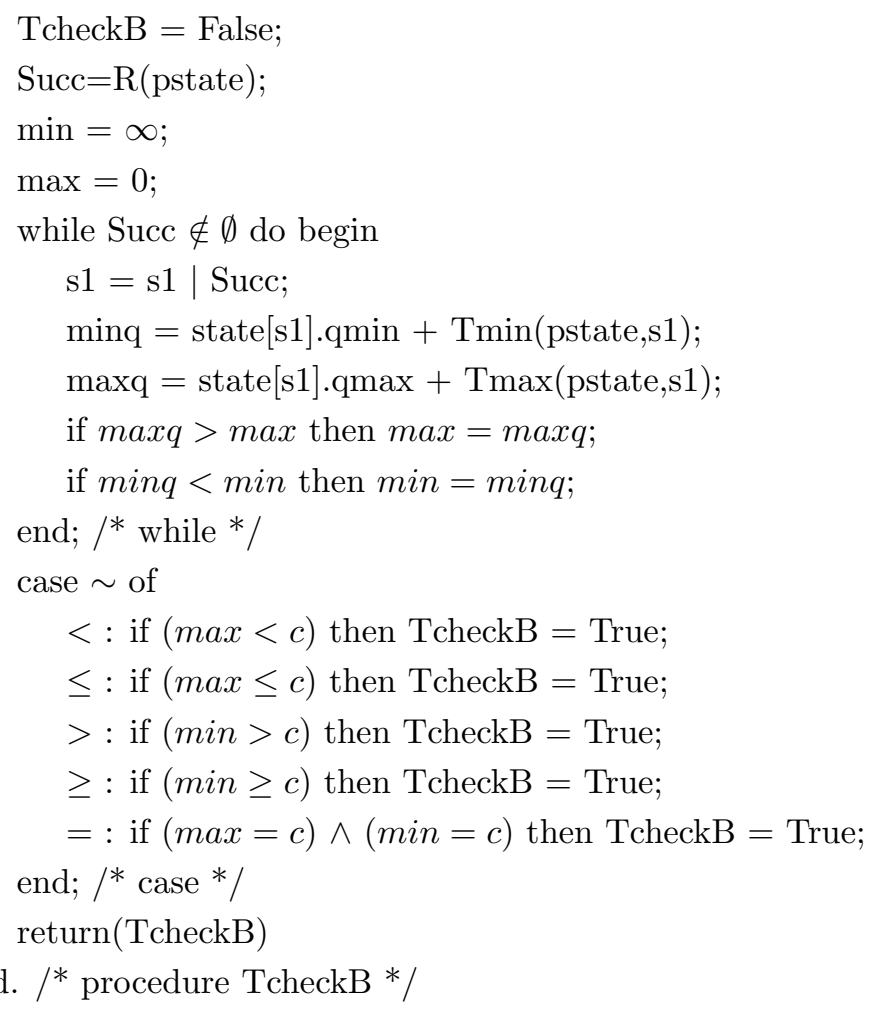

Esta operação se repete até a pilha ficar vazia. A busca é encerrada assim que todos os estados da estrutura tenham sido visitados. O algoritmo 3.5 mostra a subrotina que atualiza a variável List.

\section{Algoritmo 3.5. Subrotina UpdateList.}

proc UpdateList (cmin, cmax, s);

begin

for all $s 2 \in R(s)$ do begin

if ( $\neg$ state[s2].visited) then

if ( $\mathrm{s} 2 \notin$ List) then begin

List $=$ List $\cup\{s 2\}$;

state[s2].tmin $=\mathrm{cmin}+\operatorname{TminG}(\mathrm{s}, \mathrm{s} 2)$;

state[s2].tmax $=\mathrm{cmax}+\operatorname{TmaxG}(\mathrm{s}, \mathrm{s} 2) ;$

end

else begin

if state[s2].tmin $>$ cmin $+\operatorname{TminG}(\mathrm{s}, \mathrm{s} 2)$ then state[s2].tmin $=\operatorname{cmin}+\operatorname{TminG}(\mathrm{s}, \mathrm{s} 2)$;

if state[s2].tmax $<\operatorname{cmax}+\operatorname{TmaxG}(\mathrm{s}, \mathrm{s} 2)$ then state[s2].tmax $=\operatorname{cmax}+\operatorname{TmaxG}(\mathrm{s}, \mathrm{s} 2)$ end;

end; $/ *$ for $* /$

end.

Assumiremos que true $\in L(s)$ para todo $s \in S$. Desta forma as fórmulas do tipo $\forall \square_{\geq 0} f, \exists \square_{\geq 0} f, \forall \diamond_{\sim_{c}} f$ and $\exists \diamond_{\sim_{c}} f$ podem ser computadas de forma correta (veja a tabela 3.1 ).

A prova que estabelece o correto funcionamento da subrotina AUcheck é mostrada no apêndice B.1. 


\subsubsection{Verificando as fórmulas UNTIL com quantificador existencial}

A relação de equivalência na CTL para as fórmulas que usam o operador UNTIL com quantificador existencial é a seguinte:

$$
\exists[\phi U \varphi] \equiv \varphi \vee(\phi \wedge \exists \chi(\exists[\phi U \varphi]))
$$

Sejam $P$ e $Q$ os respectivos conjuntos de estados que satisfazem as fórmulas $\phi$ e $\varphi$. O conjunto de estados que satisfaz a fórmula $\exists \phi U \varphi$ é o limite de convergência da seqüencia de conjuntos $\left(E_{j}\right)$ definidos da seguinte forma:

$$
\begin{aligned}
E_{0} & =Q \\
E_{k+1} & =E_{k} \cup\left\{s \mid(s \in P) \wedge\left(\exists s^{\prime} \mid\left(s,-, s^{\prime}\right) \in E\right) \wedge\left(s^{\prime} \in E_{k}\right\}\right.
\end{aligned}
$$

Similarmente ao caso anterior, isto nos leva a sugerir um algoritmo para o cálculo dos estados onde a fórmula $\exists\left[p U_{\sim_{c}} q\right]$ é verdadeira.

Seja $t_{r}$ o tempo transcorrido para atingir o estado $s$ a partir de um estado predecessor e $t_{q}$ o tempo transcorrido para atingir o estado onde $q \wedge \exists\left[p U_{\sim c} q\right]$ é verdadeira. A fórmula $\exists\left[p U_{\sim_{c}} q\right]$ é válida em um estado $s \in S$ se e somente se:

$$
\underbrace{(q \in L(s))}_{A} \vee \underbrace{\left(p \in L(s) \wedge\left(\exists s^{\prime} \in R(s) \mid \exists\left[p U_{<c} q\right] \in L\left(s^{\prime}\right) \wedge\left(t_{r}+t_{q}<c\right)\right)\right)}_{B}
$$

Suponha que se queira determinar os estados que satisfazem a fórmula $\exists\left[p U_{<c} q\right]$. Neste caso, o termo A é satisfeito em todos os estados onde $q$ é verdadeiro. O termo B é satisfeito em todos os estados onde $p$ é verdadeiro, portanto existe ao menos um sucessor rotulado com a fórmula e esse estado sucessor é atingível em um tempo que satisfaz a restrição temporal. A comparação é feita usando a constante $c$ e os tempos mínimo ou máximo dependendo do tipo de relação que o símbolo $\sim$ representa.

Como especificado pela condição 3.17, basta que um destes termos seja satisfeito para que a fórmula seja declarada válida nesse estado. Como no caso anterior, o termo B requer uma busca por um estado sucessor onde a fórmula seja válida, o que nos leva novamente a sugerir um algoritmo de busca em profundidade sobre a estrutura.

A condição para que este algoritmo funcione corretamente é que as subfórmulas $p$ e $q$ estejam corretamente rotuladas em todos os estados da estrutura. 
func $\operatorname{EUcheck}(p, q, \sim, c)$

begin

for each $s \in S$ do begin

state[s].visited $=$ false;

state $[\mathrm{s}] \cdot \operatorname{tmin}=0$;

state[s].tmax $=0$

state[s].qmin $=\infty$;

state[s].qmax $=0$;

end; $/ *$ for $* /$

List $=\left\{s_{0}\right\}$;

while List $\neq \emptyset$ do begin

$\mathrm{s}=\mathrm{L} \mid$ List;

if $\neg$ state[s].visited then $\operatorname{DFSeu}(p, q, \sim, c$, state $[s]$. tmin, state $[s] . t m a x, s$, out $, 0,0)$;

end; /* while */

end. $/ *$ function EUcheck */

Como se pode ver, o algoritmo é bem similar ao algoritmo 3.2 que identifica os estados onde o operador UNTIL com quantificador universal é válido.

\section{Algoritmo 3.7. Busca em profundidade para o operador UNTIL com quantificador existencial.}

$\operatorname{proc} \operatorname{DFSeu}(p, q, \sim, c, c m i n, \operatorname{cmax}, s$, out, qmin, qmax $)$

begin

if state[s].visited then begin

if state $[s] . q \min =\infty \wedge$ state $[s] . q \max =0$ then out $=$ False

else begin

out $=$ True;

qmin $=$ state $[\mathrm{s}] \cdot$ qmin;

qmax $=$ state[s].qmax;

end;

break;

end;

state[s].visited $=$ True;

state[s].tmin $=$ cmin;

state[s].tmax $=$ cmax;

out $=$ False;

if $q \in L(s)$ then begin

out $=$ True;

$L(s)=L(s) \cup\left\{\forall\left[p U_{\sim c} q\right]\right\} ;$

UpdateList(cmin, cmax, s);

state[s].qmax $=0$;

state[s].qmin $=0$;

qmax $=0$;

$\mathrm{qmin}=0$;

break;

end;

if $p \in L(s)$ then begin

$\operatorname{push}(\mathrm{s})$;

Succ $=\mathrm{R}(\mathrm{s})$; 
if Succ $\in \emptyset$ then out $=$ False;

while Succ $\notin \emptyset$ do begin

$\mathrm{s} 1=\mathrm{s} 1 \mid$ Succ;

$\operatorname{DFS}(p, q, \sim, c, c \min +\operatorname{Tmin} G(s, s 1), \max +\operatorname{Tmax} G(s, s 1), s 1$, out1, $\operatorname{minq}, \max q)$; out $=$ out $\vee$ out 1 ;

if out then begin

if $\max q+\operatorname{Tmax} G(s, s 1)>\operatorname{state}[s] \cdot q \max$ then $\operatorname{state}[s] \cdot q \max =\max q+\operatorname{Tmax} G(s, s 1)$

if $\min q+\operatorname{Tmin} G(s, s 1)<$ state $[s] . q \min$ then state $[s] . q \min =\operatorname{minq}+\operatorname{Tmin} G(s, s 1)$; end $/ *$ if $* /$

end; /* while */

$\operatorname{pop}(\mathrm{s})$;

if out $\wedge T \operatorname{check} B(\sim, c, s)$ then

$$
L(s)=L(s) \cup\left\{\exists\left[p U_{\sim c} q\right]\right\}
$$

break;

end;

UpdateList(cmin, cmax, s);

end. $/ *$ procedure DFSeu */

A subrotina DFSeu é também similar à usada na seção anterior com a diferença de que só um dos sucessores do estado sendo verificado precisa ter sido rotulado com a fórmula para a subrotina rotular o estado corrente. A subrotina UpdateList continua a mesma mas a subrotina $T \operatorname{checkB}$ sofre uma modificação como mostrado no seguinte algoritmo.

\section{Algoritmo 3.8. Subrotina TcheckB usada pela subrotina DFSeu.}

func TcheckB $(\sim, c$, pstate $)$ : boolean;

begin

TcheckB = False;

Succ $=\mathrm{R}($ pstate);

$\min =\infty$;

$\max =0$

while Succ $\notin \emptyset$ do begin

$\mathrm{s} 1=\mathrm{s} 1 \mid$ Succ;

if state[s1].qmin $<>\infty \wedge$ state[s1].qmax $<>0$ then begin

$\operatorname{minq}=\operatorname{state}[\mathrm{s} 1] \cdot \mathrm{qmin}+\mathrm{Tmin}($ pstate, $\mathrm{s} 1) ;$

$\operatorname{maxq}=\operatorname{state}[\mathrm{s} 1] \cdot q \max +\operatorname{Tmax}($ pstate, $\mathrm{s} 1) ;$

if $\max q>\max$ then $\max =\max q$;

if $\operatorname{minq}<\min$ then $\min =\operatorname{minq}$;

end; $/ *$ if $* /$

end; /* while */

case $\sim$ of

$<:$ if $(\min <c)$ then TcheckB $=$ True;

$\leq:$ if $(\min \leq c)$ then TcheckB $=$ True;

$>:$ if $(\max >c)$ then TcheckB $=$ True;

$\geq:$ if $(\max \geq c)$ then TcheckB $=$ True;

$=:$ if $(\max =c) \wedge(\min =c)$ then TcheckB = True;

end; /* case */

return (TcheckB)

end. $/ *$ procedure TcheckB */ 
A prova do funcionamento correto deste algoritmo é bem similar à prova apresentada para o caso anterior no apêndice B.1, razão pela qual não será apresentada aqui.

\subsubsection{Verificando as fórmulas com o operador de diferença de tempo}

Para verificar fórmulas que contém o operador de diferença de tempo é preciso um algoritmo que determine a menor e a maior diferença de tempo entre conjuntos de estados. Os conjuntos de estados são determinados como os estados que satisfazem a fórmula $\phi_{1}$ e os que satisfazem a fórmula $\phi_{2}$. Como foi dito antes, estes conjuntos devem ser não vazios e disjuntos. Após a diferença de tempo entre os conjuntos ter sido determinada, o menor valor, o maior valor, ou ambos, são comparados com a constante usada como restrição temporal de acordo com a semântica do operador definida na tabela 3.2.

O algoritmo 3.9 é dividido em etapas. A primeira delas determina que estados correspondem a cada conjunto, inicializando o contador de tempo máximo e mínimo de cada um deles em zero e marcando cada um desses estados como visitados. Nesta mesma etapa as condições necessárias para a existência da diferença de tempo são verificadas. A segunda etapa se encarrega de determinar a diferença entre os conjuntos de estados. A terceira faz a comparação dos valores obtidos com a constante $c$ e determina a validade da fórmula.

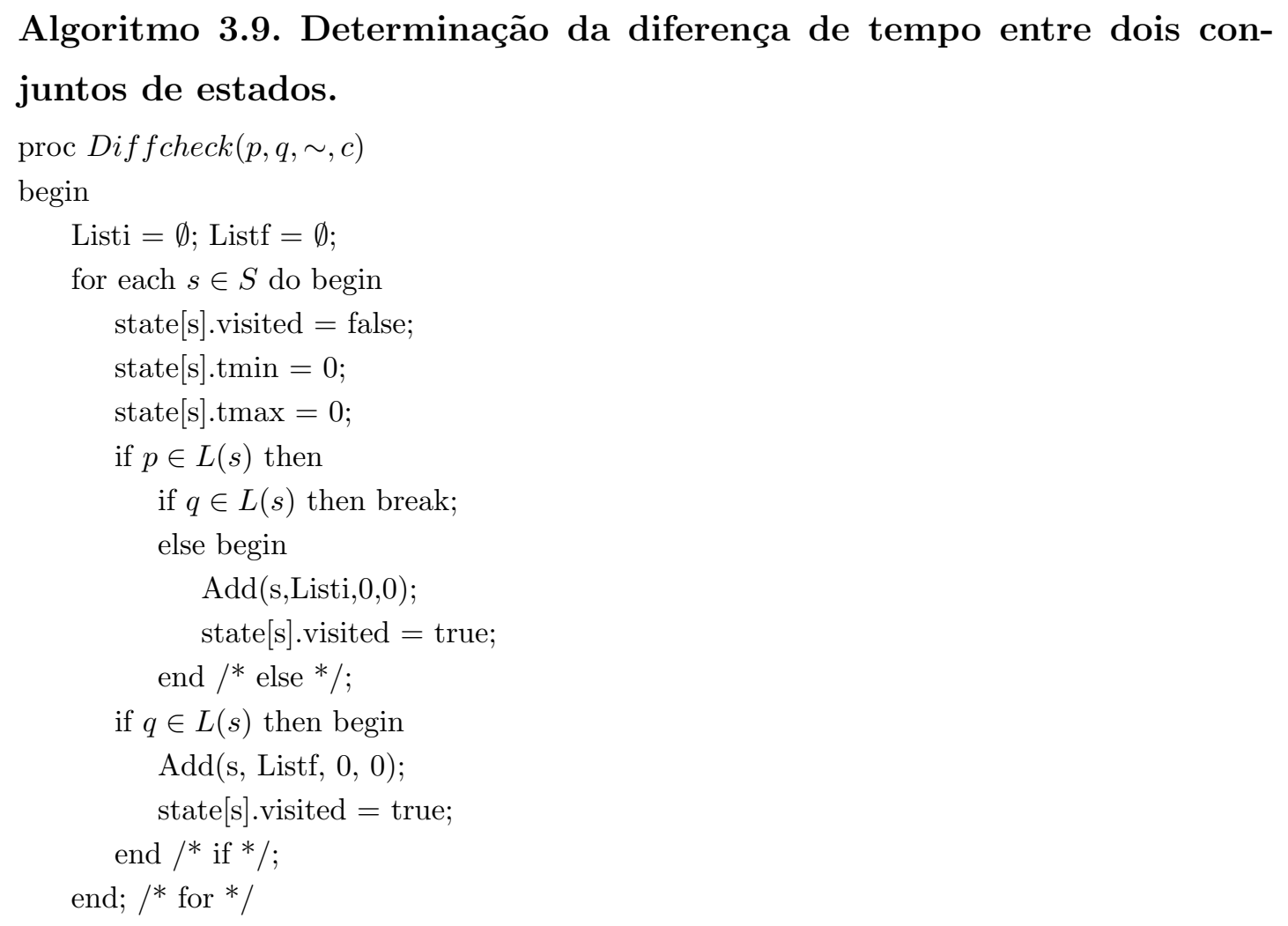




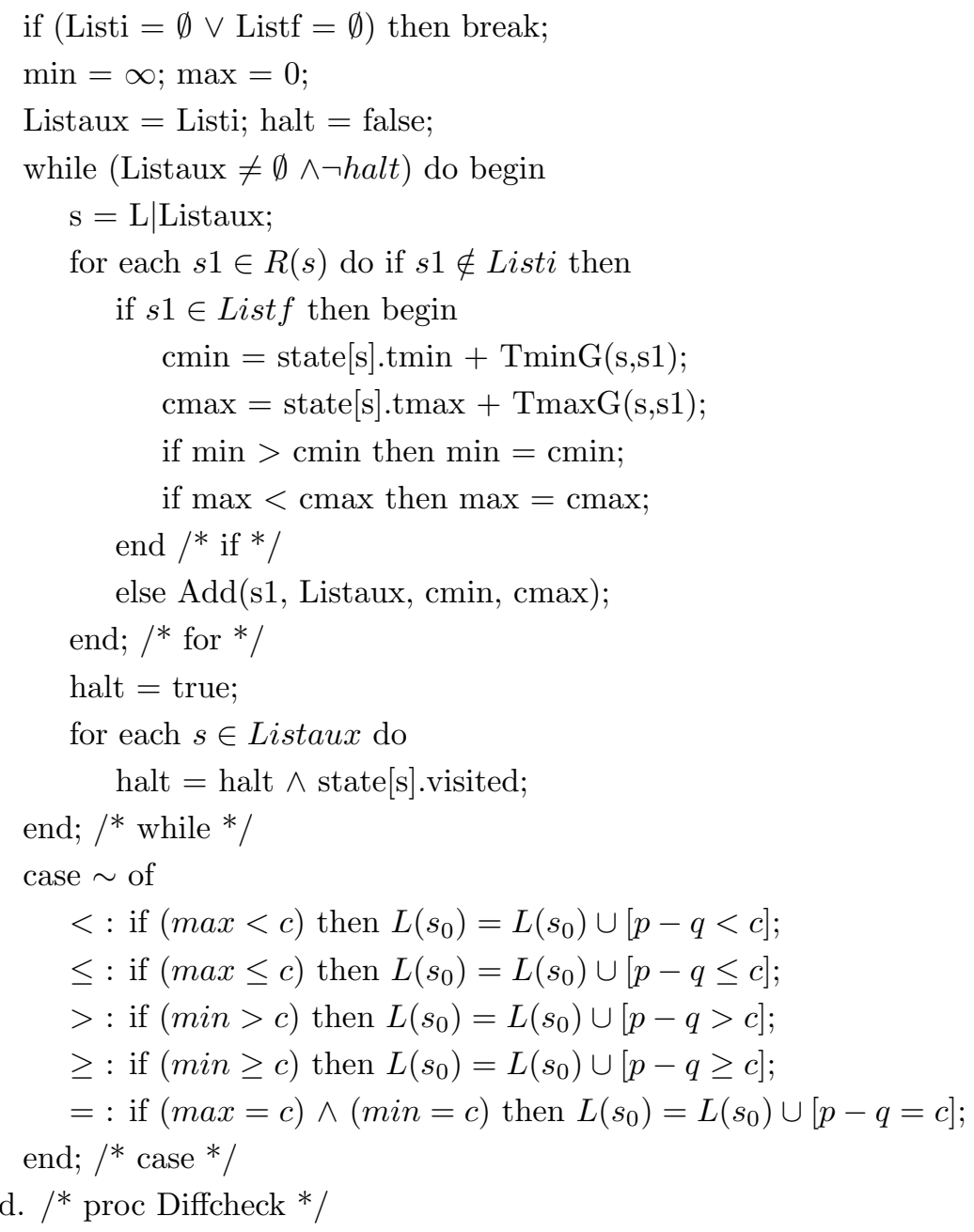

A segunda etapa deste algoritmo é a mais importante pelo seu decisivo papel na complexidade. A seguir discute-se as idéias por trás deste algoritmo.

A busca começa pelos estados que pertencem ao conjunto que satisfaz $\phi_{1}$, armazenados na variável Listaux. Cada elemento deste conjunto é retirado um a um, e substituído pelos sucessores dos estados que não pertençam ao conjunto inicial, atualizando os respectivos tempos mínimo e máximo para atingir tais estados. Se estes estados sucessores pertencem a conjunto dos estados finais (estados que satisfazem $\phi_{2}$ ), então não precisam ser colocados em Listaux e dado que atingiram algum dos estados objetivo os tempos máximo e mínimo são atualizados.

Como a variável Listaux é modificada a cada execução do ciclo, a condição de parada é alcançada de dois modos:

- O conjunto auxiliar denotado pela variável Listaux fica vazio, o que somente pode acontecer quando todos os sucessores dos estados que se encontravam no passo anterior atingiram estados do conjunto final e portanto todas as variantes possíveis já foram contempladas. 
- Todos os estados presentes na variável Listaux já foram visitados e portanto nenhum sucessor que não tenha sido contemplado poderá modificar o conjunto. Nesta situação os estados que poderiam levar a estados objetivo já foram contemplados.

O algoritmo 3.10 mostra o funcionamento da subrotina Add usada para construir as listas usadas pela subrotina Diffcheck.

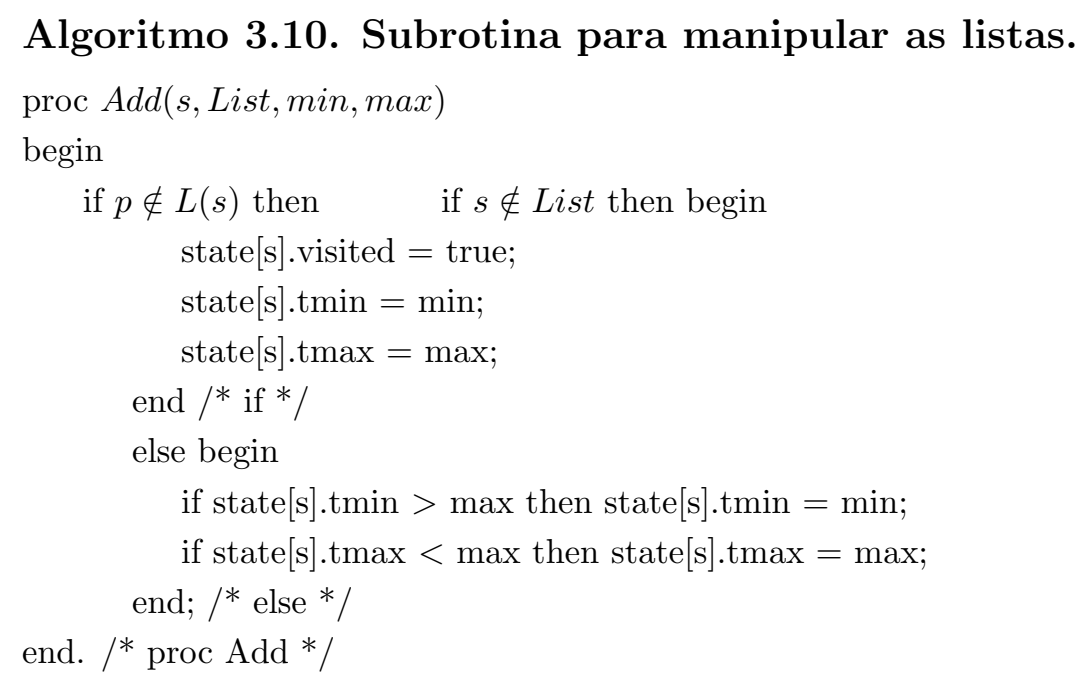

\subsubsection{A verificação de modelos de forma local}

Intuitivamente sabemos que o problema da verificação local requer menos recursos que o problema global pois não é necessário rotular todos os estados. Se a fórmula contém só sub-fórmulas de estados o algoritmo é bem simples, restringindo-se a avaliar a fórmula no estado para o qual a verificação foi solicitada. Se a fórmula envolve fórmulas de caminhos o problema é mais complexo pois não é possível determinar "a priori" quantos estados sucessores devem ser rotulados para poder determinar a validade da fórmula.

O algoritmo denominado ALMC apresentado em (HELJANKO, 1997) ${ }^{7}$ para verificar a CTL, marca todas as sub-fórmulas em cada estado com o valor "desconhecido" e vai rotulando as sub-fórmulas uma por vez mudando o seu estado para verdadeiro ou falso.

O problema de um algoritmo como este é que precisa o dobro de espaço para armazenar o valor das sub-fórmulas em cada estado ${ }^{8}$. Também precisa de

\footnotetext{
${ }^{7}$ Este algoritmo é baseado em um outro algoritmo para verificação local proposto por Vergauwen e Lewi (1993)

${ }^{8}$ Note que dois estados possíveis podem ser representados com 1 bit, três estados precisam de 2 bits.
} 
uma varredura mais do espaço de estados para marcar as sub-fórmulas com valor desconhecido antes de executar de fato a verificação da fórmula.

A proposta de algoritmo do autor desta tese está dividida em duas partes: uma que verifica fórmulas de estados e outra que verifica as formulas de caminho. Esta última verifica primeiro as fórmulas de estado em todos os estados e depois verifica a fórmula de caminho.

Desta forma o algoritmo proposto economiza espaço, mas perde no tempo de execução médio. Na análise de pior caso, a complexidade permanece a mesma que a de outros autores, como será mostrado na seção 3.4.4. O algoritmo 3.11 verifica as fórmulas de estados e o algoritmo 3.12 verifica as fórmulas que possuem pelo menos uma fórmula de caminho. A variável stack é uma variável tipo pilha, que contém as sub-fórmulas de $\phi^{9}$.

\section{Algoritmo 3.11. Verificação de sub-fórmulas de estados para o pro- blema da verificação local .}

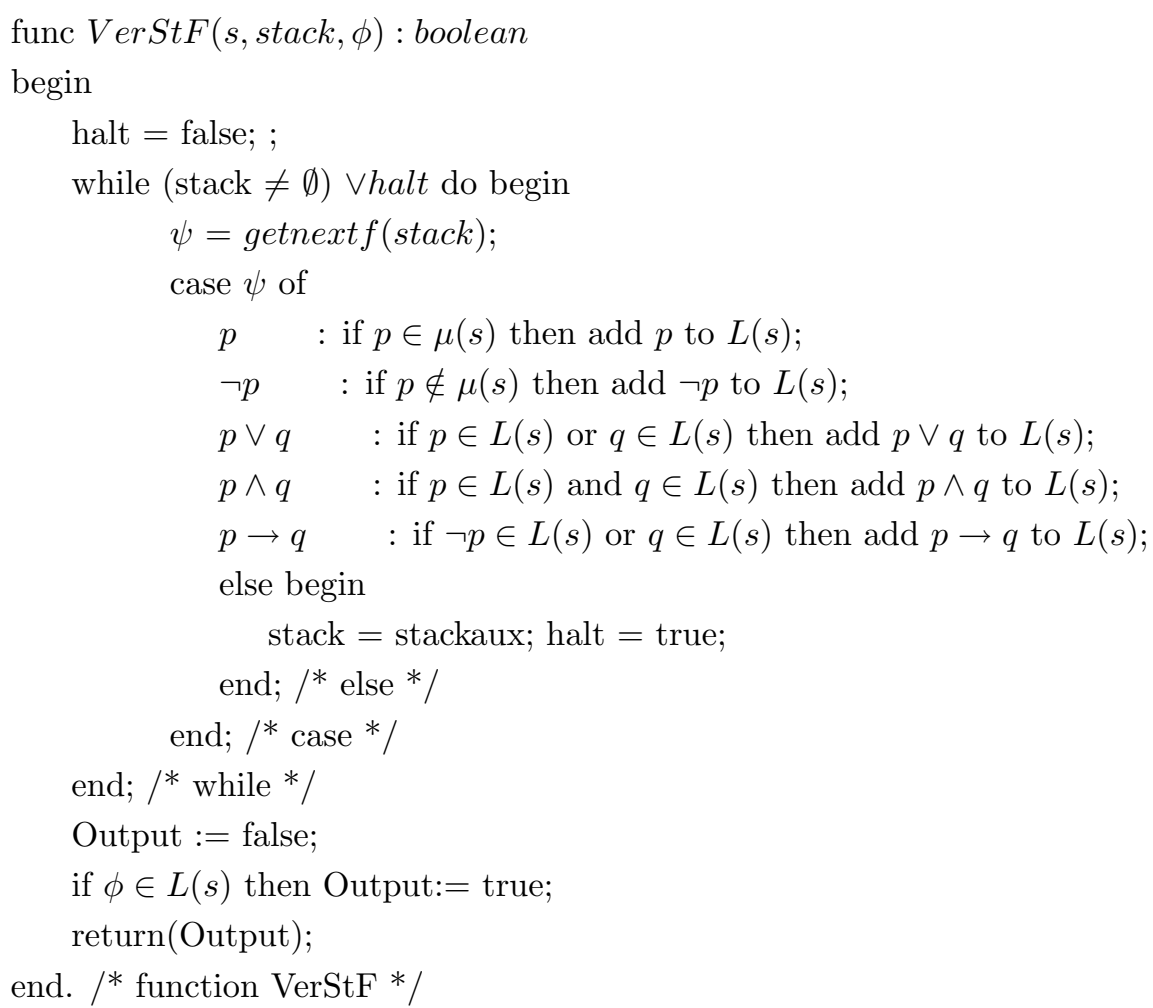

O funcionamento correto do algoritmo 3.11 depende das sub-fórmulas de $\phi$ estarem na ordem correta, segundo especificado na seção 3.4.1. Se todas as subfórmulas em que $\phi$ é decomposta são fórmulas de estados, a subrotina $\operatorname{VerStF}$ terminará com o stack vazio e a validade da fórmula $\phi$ será devidamente verificada. No caso de existir alguma sub-fórmula de caminho no stack este é colocado no seu estado inicial e a função retorna com valor falso.

\footnotetext{
${ }^{9}$ a fórmula $\phi$ está inclusa no fundo da pilha.
} 
O algoritmo 3.12 utiliza a função VerStF para determinar se a fórmula contém sub-fórmulas de caminho. Se o stack estiver vazio a fórmula só contém sub-fórmulas de estado e o problema da verificação local já foi resolvido. Se o stack não está vazio é porque existem sub-fórmulas de caminho em $\phi$ e uma subrotina precisa verificá-las.

\section{Algoritmo 3.12. Verificação de fórmulas TCTL em um estado específico do GTT.}

func LocalMCheck (st, stack, $\phi)$ : boolean

begin

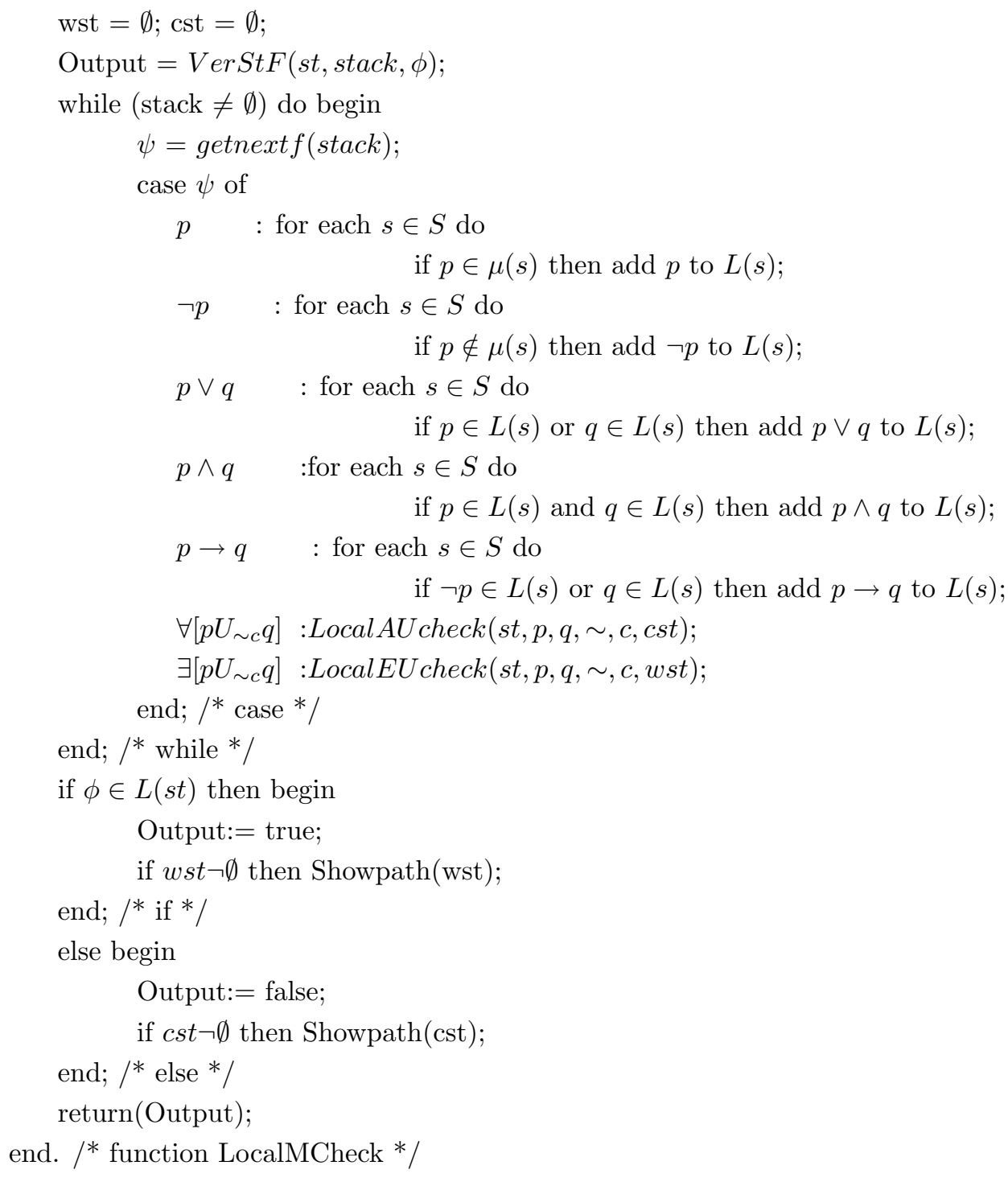

Antes de verificar as fórmulas de caminho em $\phi$ as fórmulas de estado são verificadas em todos os estados do GTT. Isto elimina a necessidade de rotular ;um estado como "desconhecido" para as sub-fórmulas de $\phi$ e portanto elimina também uma varredura no espaço de estados para colocar estas sub-fórmulas nesse estado. Por outro lado, uma varredura completa do espaço de estados é introduzida para cada sub-fórmula de estado em $\phi$. 
A estratégia para a verificação das fórmulas de caminho em $\phi$ é a mesma usada em (HELJANKO, 1997). No caso da fórmula com quantificador universal o algoritmo procura um caminho onde a fórmula seja falsa. Este caminho, se existe, é chamado de contra-exemplo. Para o caso da fórmula com quantificador existencial procura-se um caminho onde a fórmula seja verdadeira, se existir, tal caminho é chamado de avalista.

A seguir discute-se estes algoritmos em detalhes, além de formalizar as definições de contra-exemplos e avalistas.

\subsubsection{Verificando as fórmulas $U N T I L$ com quantificador universal}

Para formular a definição de contra-exemplo a ser usada na verificação de fórmulas com quantificador universal podemos partir da seguinte relação de equivalência da lógica CTL:

$$
\forall[p U q] \equiv \neg(\exists[\neg q U \neg p \wedge \neg q] \vee \exists \square(\neg q))
$$

Se for incluída a restrição temporal no operador UNTIL a definição de contraexemplo deverá seguir a seguinte relação de equivalência:

$$
\begin{aligned}
\forall\left[p U_{<c} q\right] & \equiv \neg\left(\exists\left[\neg q U_{<c} \neg p \wedge \neg q\right] \vee \exists \square(\neg q)\right) \\
\forall\left[p U_{\leq c} q\right] & \equiv \neg\left(\exists\left[\neg q U_{\leq c} \neg p \wedge \neg q\right] \vee \exists \square(\neg q)\right) \\
\forall\left[p U_{>c} q\right] & \equiv \neg\left(\exists\left[\neg q U_{>c} \neg p \wedge \neg q\right] \vee \exists \square(\neg q)\right) \\
\forall\left[p U_{\geq_{c}} q\right] & \equiv \neg\left(\exists\left[\neg q U_{\leq c} \neg p \wedge \neg q\right] \vee \exists \square(\neg q)\right) \\
\forall\left[p U_{=c} q\right] & \equiv \neg\left(\exists\left[\neg q U_{=c} \neg p \wedge \neg q\right] \vee \exists \square(\neg q)\right)
\end{aligned}
$$

A definição de contra-exemplo estará ligada ao tipo de relação binária usada na restrição temporal. Portanto pode-se enunciar a seguinte definição:

Definição 23 (contra-exemplo, $<$ ). Um caminho $\sigma_{c}=s_{0} \stackrel{a_{1}, d_{1}}{\longrightarrow} s_{1} \stackrel{a_{2}, d_{2}}{\longrightarrow} \ldots \stackrel{a_{n}, d_{n}}{\longrightarrow}$ $s_{n}$ é um contra-exemplo da fórmula $\forall\left[p U_{<c} q\right]$ no estado $s_{0}$, na estrutura $G$, sse:

$$
\begin{gathered}
\exists i\left[i \geq 0 \wedge G, s_{i}=(\neg p \wedge \neg q) \wedge \forall j\left[0 \leq j<i \Rightarrow G, s_{j}=\neg q\right] \wedge \sum_{x=0}^{i} d_{x}<c\right] \quad, \text { ou } \\
\exists i\left[i \geq 0 \wedge \forall j\left[0 \leq j<i \Rightarrow G, s_{j} \models \neg q\right]\right]
\end{gathered}
$$

onde $i, j \in[1 . . n]$ e $d_{x} \in R^{+}$.

O algoritmo para determinar a validade da fórmula $\forall\left[p U_{\sim_{c}} q\right]$ em um determinado estado st procura um contra-exemplo que comece neste estado. 
Algoritmo 3.13. Subrotina para verificar fórmulas com o operador $U N$ TIL com quantificador universal.

proc LocalAUcheck(st, $p, q, \sim, c, c s t)$

begin

try

state[st].tmin $=0$;

state[st].tmax $=0$;

if $(\neg q \in L(s t) \wedge \neg p \in L(s t))$ then begin

$\operatorname{push}($ st,cst);

throw abort;

end; $/ *$ if $* /$

if $q \in L(s t)$ then begin

$L(s t)=L(s t) \cup \forall\left[p U_{\sim_{c}} q\right] ;$

throw abort;

end; $/ *$ if $* /$

Succ $=\mathrm{R}(\mathrm{st})$;

while Succ $\notin \emptyset$ do begin

$\mathrm{s} 1=\mathrm{s} 1 \mid$ Succ;

$\mathrm{cmin}=\min +\operatorname{Tmin}(\mathrm{st}, \mathrm{s} 1) ;$

$\operatorname{cmax}=\max +\operatorname{Tmax}(\mathrm{st}, \mathrm{s} 1) ;$

state[s1].tmin $=\mathrm{cmin}$;

state[s1].tmax $=$ cmax;

SearchCE $(s 1, p, q$, cmin, cmax $, \sim, c, c s t) ;$

end; /* while */

$L(s t)=L(s t) \cup \forall\left[p U_{\left.\sim_{c} q\right]} ;\right.$

catch abort

/* Nada a fazer, cst aponta ao contra-exemplo */

end. ${ }^{*}$ procedure LocalAUcheck */

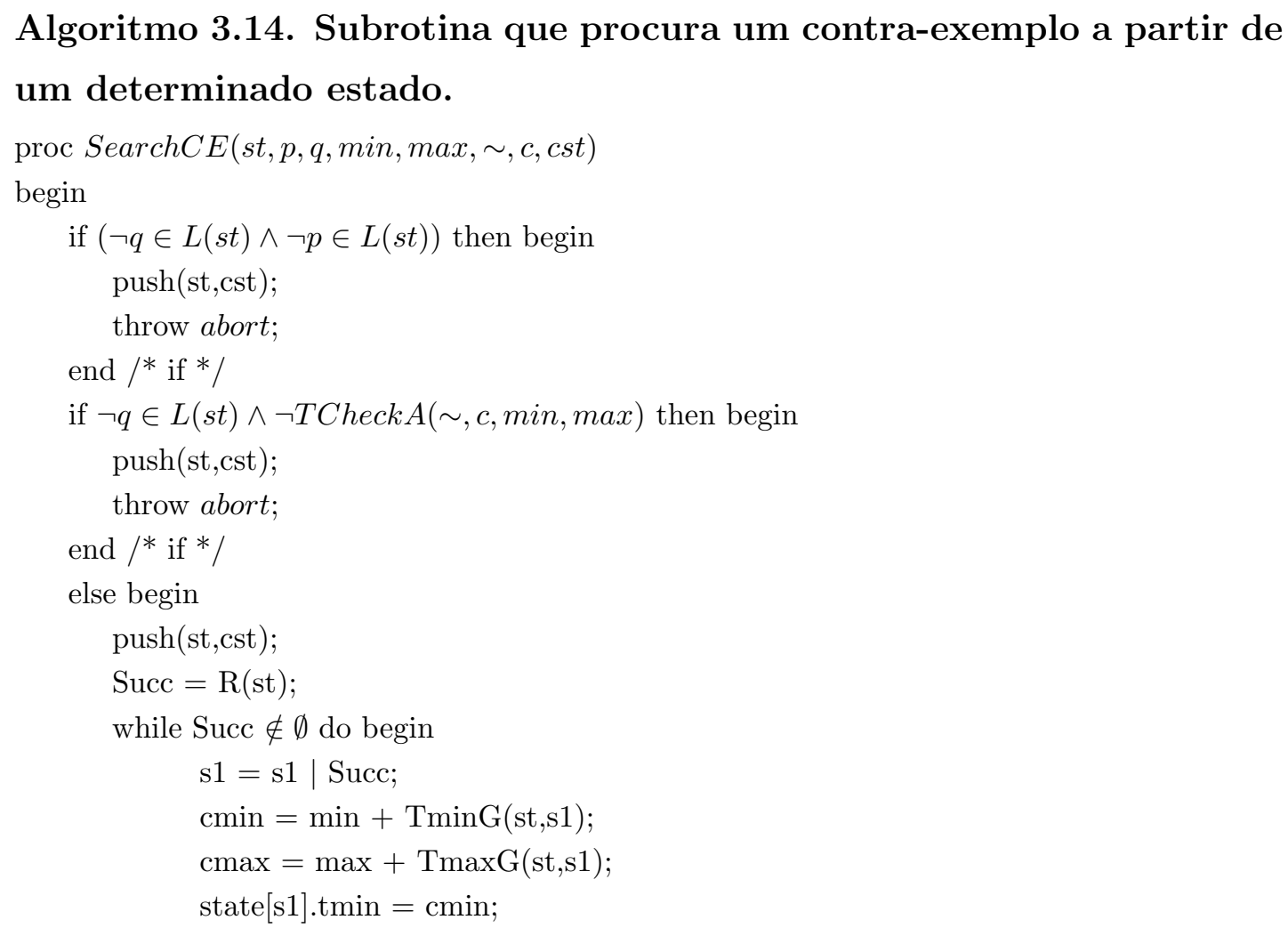


state[s1].tmax $=$ cmax;

SearchCE $(s 1, p, q$, cmin, cmax $, \sim, c, c s t)$;

end; /* while */

$\operatorname{pop}(\mathrm{cst})$;

end $/ *$ else $* /$

end. /* procedure SearchCE */

\subsubsection{Verificando as fórmulas UNTIL com quantificador existencial}

A definição de caminho do tipo avalista deriva diretamente da semântica do operador UNTIL com quantificador existencial. Similarmente ao caso da definição de contra-exemplo, esta definição é dependente da relação binária usada na restrição temporal.

Definição 24 (avalista, $<$ ). Um caminho $\sigma_{w}=s_{0} \stackrel{a_{1}, d_{1}}{\longrightarrow} s_{1} \stackrel{a_{2}, d_{2}}{\longrightarrow} \ldots \stackrel{a_{n}, d_{n}}{\longrightarrow} s_{n}$ é um avalista para a fórmula $\exists\left[p U_{<c} q\right]$ no estado $s_{0}$, da estrutura $G$ sse:

$$
\exists i\left[i \geq 0 \wedge G, s_{i} \models q \wedge \forall j\left[0 \leq j<i \Rightarrow G, s_{j} \models p\right] \wedge \sum_{x=0}^{i} d_{x}<c\right]
$$

onde $i, j \in[1 . . n]$ e $d_{x} \in R^{+}$.

O algoritmo para determinar a validade da fórmula $\exists\left[p U_{\sim_{c}} q\right]$ em um determinado estado st procura por um avalista que comece neste estado.

\section{Algoritmo 3.15. Subrotina para verificar fórmulas com o operador $U N$ - TIL com quantificador existencial.}

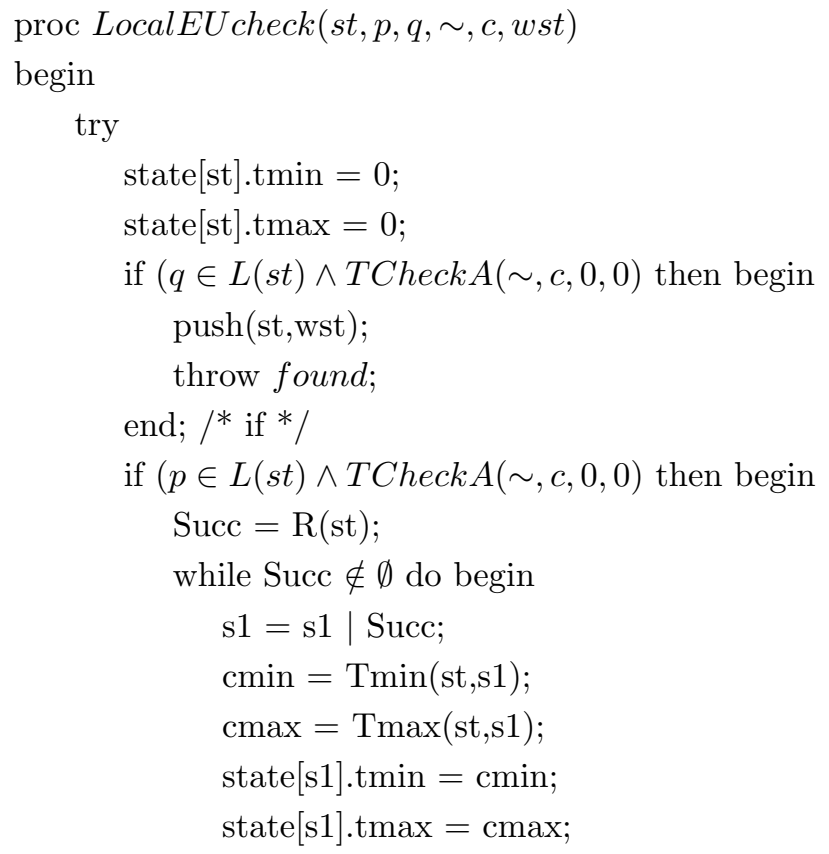


$\operatorname{Search} W(s 1, p, q, \operatorname{cmin}, \operatorname{cmax}, \sim, c, w s t) ;$

end; /* while */

end; $/ *$ if $* /$

else break;

catch found

$L(s t)=L(s t) \cup \exists\left[p U_{\sim_{c}} q\right] ;$

end. /* procedure LocalEUcheck */

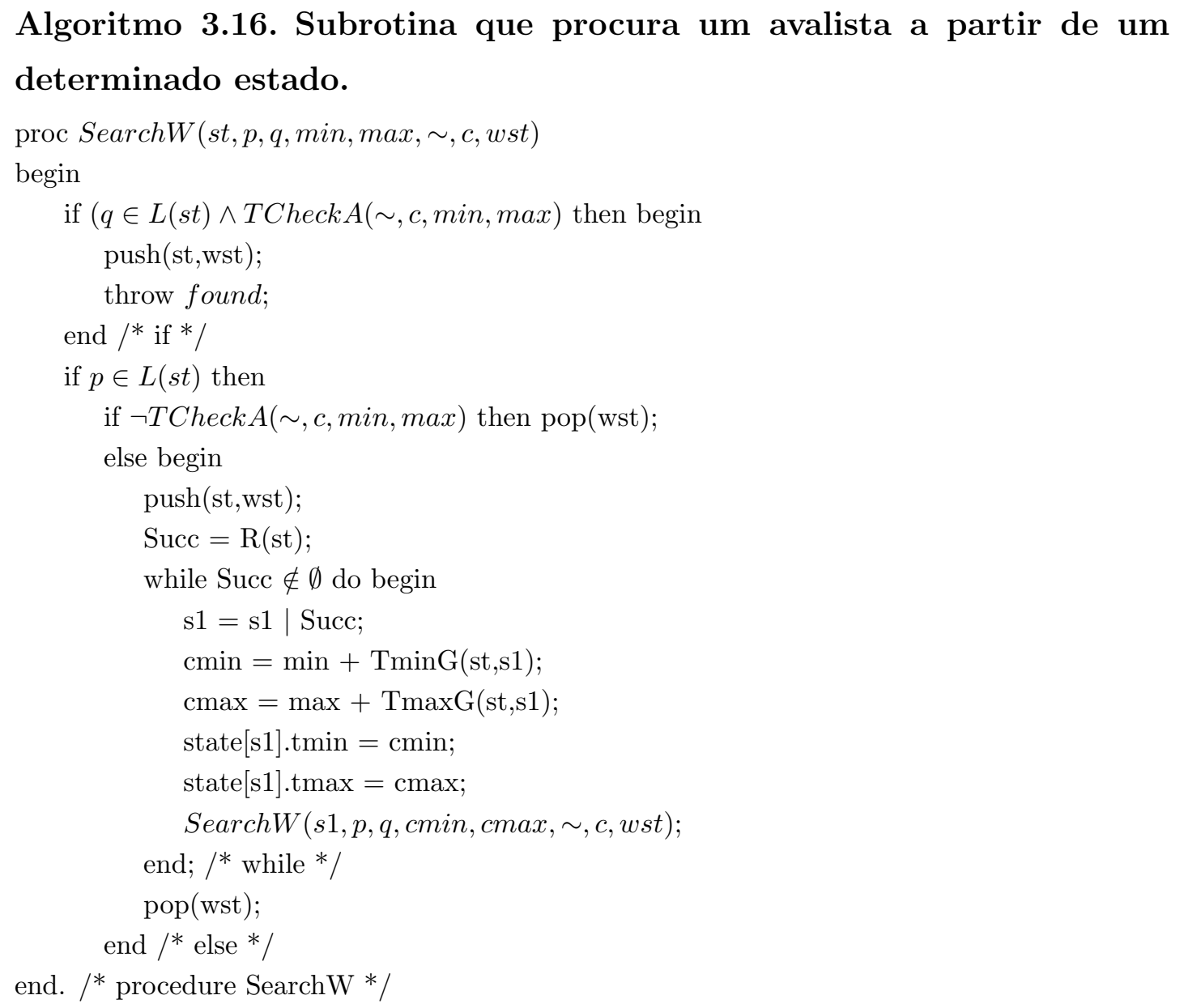

\subsubsection{Análise dos requisitos de tempo e espaço}

Nesta seção discute-se a complexidade dos procedimentos de verificação aqui propostos. Lembre-se que a complexidade aqui tratada refere-se à verificação de fórmulas TCTL sobre uma estrutura, e portanto o custo na construção desta estrutura não será levado em consideração. Desta forma pode-se realizar a comparação da complexidade destes algoritmos com outros existentes na literatura ainda que para a lógica CTL.

A seguir, apresentam-se os resultados na análise dos requisitos de tempo e espaço dos algoritmos aqui propostos para cada problema (global e local). 
Teorema 25 (Complexidade temporal do algoritmo de verificação global). Existe um algoritmo que determina a validade de uma fórmula TCTL $\phi$ em todos os estados $s \in S$ da estrutura $G=(S, \mu, s, A, E, I t)$ o qual é executado em tempo $O($ length $(\phi) \times(\operatorname{card}(S)+\operatorname{card}(E)))$.

Prova A complexidade do algoritmo de verificação depende da complexidade dos algoritmos AUCheck, EUCheck and DiffCheck (são os algoritmos de maior complexidade entre aqueles encarregados de avaliar as sub-fórmulas que formam a sintaxe da TCTL), e da cardinalidade do conjunto das sub-fórmulas em que a fórmula $\phi$ pode ser decomposta, que é equivalente ao comprimento da fórmula (Definição 22). O tempo usado pela subrotina AUCheck depende do número de vezes que o procedimento $D F S$ é chamado. Uma chamada a $D F S$, excluindo o tempo usado nas chamadas recursivas, demora um tempo constante mais um tempo proporcional ao número de sucessores do estado que está sendo processado (é igual ao número de arestas que saem desse estado). O número de vezes que $D F S$ é chamado, seja pelo $A U C h e c k$, seja de forma recursiva, é igual ao número de estados no GTT uma vez que cada estado é visitado uma vez só. Portanto podemos concluir que $A U C h e c k$ demora um tempo proporcional ao número de estados mais o número de arestas ou seja, $O((\operatorname{card}(S)+\operatorname{card}(E)))$. O algoritmo EUCheck é bem similar a AUCheck porém este não precisa procurar em todos os estados para determinar a validade da fórmula. De qualquer forma, no pior caso, precisaria percorrer todos os estados, mantendo uma complexidade igual à de $A U C h e c k$. No caso de DiffCheck, o pior caso ${ }^{10}$ depende do número de estados no GTT após a construção das listas inicial e final assim como da determinação das condições necessárias, e do número de arestas devido a busca dos caminhos de menor e maior peso entre as duas listas. Portanto, podemos concluir que a maior complexidade das sub-fórmulas a verificar é $O((\operatorname{card}(S)+\operatorname{card}(E)))$.

Levando em consideração que o número de sub-fórmulas a verificar até chegar a fórmula $\phi$ é igual ao comprimento de $\phi$, podemos concluir que a complexidade do algoritmo de verificação para fórmulas TCTL é $O(\operatorname{length}(\phi) \times(\operatorname{card}(S)+$ $\operatorname{card}(E)))$.

Teorema 26 (Requisito de espaço do algoritmo de verificação global). Existe um algoritmo que determina a validade de uma fórmula TCTL $\phi$ em todos os estados $s \in S$ da estrutura $G=(S, \mu, s, A, E, I t)$ e que precisa $O(($ length $(\phi)+$

\footnotetext{
${ }^{10} \mathrm{O}$ pior caso acontece quando o conjunto $p$ é formado pelo estado inicial e $q$ contém o estado final
} 
1) $\times \operatorname{card}(S)+4 \times \operatorname{card}(E))$ de espaço de memória.

Prova O espaço de memória usado para armazenar a validade de cada subfórmula de $\phi$, incluindo a própria $\phi$, para todos os estados, é determinada pelo número de estados e pelo comprimento da fórmula. O array usado para marcar os estados como visitados consome um bit para cada estado no GTT. Os valores máximos e mínimos de tempo em cada transição consomem memória proporcional ao quádruplo do número de arestas no GTT. A memória usada pelo procedimento de busca em profundidade consome $O(1)$. Sendo assim, o requisito de memória no pior caso é $O(($ length $(\phi)+1) \times \operatorname{card}(S)+4 \times \operatorname{card}(E))$.

Teorema 27 (Complexidade temporal do algoritmo de verificação local). Existe um algoritmo que determina a validade de uma fórmula TCTL $\phi$ em um estado $s_{0} \in S$ da estrutura $G=(S, \mu, s, A, E, I t)$ e que é executado em tempo $O($ length $(\phi) \times(\operatorname{card}(S)+\operatorname{card}(E)))$.

Prova A complexidade do algoritmo de verificação depende da complexidade dos algoritmos LocalAUCheck e LocalEUCheck, e da cardinalidade do conjunto das sub-fórmulas em que a fórmula $\phi$ pode ser decomposta, que é igual ao comprimento da fórmula (Definição 22). O pior caso acontece quando precisamos determinar a validade de uma fórmula no estado inicial e daí procurar em todos os estados para avaliar a validade da fórmula $\phi$. Neste caso chegamos a uma complexidade igual a do procedimento $A U C h e c k$, ou seja $O(\operatorname{card}(S)+\operatorname{card}(E))$. Se todas as sub-fórmulas de $\phi$ são fórmulas de caminho então o complexidade do algoritmo de verificação local para fórmulas TCTL é $O(l$ ength $(\phi) \times(\operatorname{card}(S)+\operatorname{card}(E)))$.

Teorema 28 (Requisito de espaço do algoritmo de verificação local). Existe um algoritmo que determina a validade de uma fórmula TCTL $\phi$ em um estado $s_{0} \in S$ da estrutura $G=(S, \mu, s, A, E, I t)$ e que precisa $O(($ length $(\phi)+$ $2) \times \operatorname{card}(S)+4 \times \operatorname{card}(E))$ de espaço de memória.

Prova O pior cenário de uso de memória neste algoritmo é atingido quando uma fórmula formada por sub-fórmulas de caminho aninhadas precisam ser verificada e o contra-exemplo ou o avalista possui comprimento igual ao caminho mais longo no GTT. Neste caso, o espaço de memória para armazenar a validade 
de cada sub-fórmula de $\phi$, incluindo a própria fórmula $\phi$, é determinado pelo comprimento da fórmula e pelo número de estados. O array usado para marcar os estados como visitados consome um bit para cada estado no GTT. Os valores máximos e mínimos de tempo em cada transição consome memória proporcional ao quádruplo do número de arestas no GTT. A memória usada pelo procedimento de busca em profundidade consome $O(1)$ e o stack que guarda o contraexemplo ou o avalista usa espaço proporcional ao tamanho do grafo $O(|S|)$. Sendo assim, o requisito de memória no pior caso é $O(($ length $(\phi)+2) \times \operatorname{card}(S)+4 \times \operatorname{card}(E))$.

Como em (ALUR; COURCOUBETIS; DILL, 1993), a mudança introduzida para verificar a lógica TCTL não aumentou de forma significativa a complexidade do algoritmo de verificação.

No anexo B é apresentada uma análise comparativa entre algoritmos para verificação de modelos usando CTL retirada de (HELJANKO, 1997). Note que a verificação da CTL é feita sobre uma estrutura $M=(S, R, P)$ onde $R$ representa a relação entre os estados, ou seja, as transições, o que equivale ao conjunto $E$ definido na estrutura proposta neste trabalho.

A complexidade destes algoritmos é bem similar, como se pode deduzir da tabela B.1 e dos teoremas aqui enunciados. Entretanto, a análise apresentada para o requisito de espaço de memória não é suficientemente detalhada para permitir uma comparação precisa. Isto se deve ao fato de que na análise do requisito de espaço de memória assumimos que o espaço necessário para armazenar a validade das sub-fórmulas é igual ao espaço usado para armazenar a relação de sucessão dos estados. Este tipo de simplificação é usual na análise de complexidade de algoritmos e é a mesma simplificação usada para obter os resultados da tabela B.1.

Uma análise mais rigorosa foi apresentada nas tabelas B.2 e B.3 com respeito ao requisito de espaço de memória dos algoritmos comparados em (HELJANKO, 1997). Uma análise mais detalhada do espaço usado pelos algoritmos local e global propostos neste trabalho, seguindo os mesmos critérios usados em (HELJANKO, 1997), é mostrada na tabela 3.6.

O aumento na quantidade de bits usados para verificar uma fórmula devese basicamente ao fato de que, na abordagem proposta, estamos lidando com domínio temporal denso. O mesmo é representado através dos intervalos definidos usando o domínio dos números racionais. Estes números são armazenados em 


\begin{tabular}{|l|l|l|l|}
\hline Algoritmo & Mapa de bits & Cons. de stack & Sucessores e predecessores \\
\hline global & $|S| \times(|\phi|+65)$ & $O(|S|)$ & $128 \times(|R| \times\lceil\log |S|\rceil+|S| \times\lceil\log |R|\rceil)$ \\
\hline local & $|S| \times(|\phi|+65)$ & $O(|S|)$ & $128 \times(|R| \times\lceil\log |S|\rceil+|S| \times\lceil\log |R|\rceil)$ \\
\hline
\end{tabular}

Tabela 3.6: Requisito de espaço de memória em bits dos algoritmos de verificação global e local.

registros de 32 bits $^{11}$.

Isto leva a uma representação da relação de estados sucessores através de uma lista de adjacência, com os seus respectivos valores de tempo, que necessita $128 \times(|R| \times\lceil\log |S|\rceil+|S| \times\lceil\log |R|\rceil)$ bits. O array que guarda o estado das subfórmulas, incluindo a fórmula verificada, precisa $|\phi| \times|S|$ bits. Os dois contadores de tempo máximo e mínimo usados nos algoritmos que verificam sub-fórmulas de caminho necessitam $2 \times 32 \times|S|$ bits e finalmente $|S|$ bits, um bit por estado, para marcar os estados que já foram visitados pelos algoritmos de busca em profundidade. O consumo de stack é determinado pelo maior comprimento de um caminho no GTT que no pior caso é o tamanho do próprio grafo, portanto é função da cardinalidade do conjunto $S$.

\footnotetext{
${ }^{11}$ consideramos 32 bits um tamanho suficiente para a representação dos intervalos de tempo, usando aritmética de ponto fixo.
} 


\section{A rede GHENeSys}

As redes de Petri têm sido utilizadas com sucesso na modelagem de sistemas discretos em diferentes áreas do conhecimento. A disseminação do uso das redes em diversas áreas levou ao aparecimento de várias extensões seja para inserir elementos típicos da aplicação seja por necessidade do processo de modelagem e design. Ao mesmo tempo, apareceram ferramentas com o intuito de automatizar o processo de modelagem e facilitar o uso prático destas extensões em problemas cada vez mais complexos.

Recentemente surgiu a idéia de uma abordagem unificada que poderia integrar todos os recursos disponíveis nas extensões que cobrem todas as necessidades gerais de modelagem e podem lidar com a maior parte, se não com a totalidade, dos sistemas discretos, qualquer que seja sua área de aplicação. Entretanto, embora esta abordagem integradora seja quase consenso entre os pesquisadores da área, nenhuma ferramenta prática foi desenvolvida neste sentido.

A rede GHENeSys foi concebida e desenvolvida a partir de idéias apresentadas em (MIYAGI, 1988), (SILVA; MIYAGI, 1995), (SILVA; MIYAGI, 1996), (SILVA, 1998) e finalmente formalisada em (FOYO, 2001). Inicialmente, a idéia era ter uma rede estendida com conceitos de orientação a objetos e dotada de mecanismos de abstração - através da uma definição de hierarquia - e de síntese - usando uma abordagem estruturada associada ao encapsulamento introduzido pelos objetos.

Potencialmente a rede GHENeSys pode vir a se tornar uma ferramenta capaz de representar de forma unificada as redes de Petri e suas extensões assim como as redes de alto nível. Uma formulação mais completa como esta depende essencialmente da escolha de um formalismo amplamente aceito para os objetos e suas relações, incluindo entre eles as marcas. Nesta tese não discutiremos a rede GHENeSys em detalhes mas apenas a evolução do formalismo original para a representação de comportamentos temporais. 


\subsection{O tempo na rede GHENeSys}

Originalmente, o comportamento temporal de sistemas modelados usando a rede GHENeSys apenas permitiam representar processos com durações determinísticas. Estes lapsos de tempo eram representados como tempo de espera no disparo de atividades, proporcionando a representação de comportamentos temporais tipo Ramchandani. A duração das atividades era um atributo das classes do tipo Atividade à qual pertencem todas as transições da rede GHENeSys e interferiam no comportamento e propriedades da rede apenas por inibirem as transições por um determinado lapso de tempo.

No entanto, para permitir a representação de sistemas com tempo onde a duração dos processos não é determinística é preciso utilizar intervalos com limites mínimo e máximo para o disparo de uma transição, como acontece nas RPT de Merlin. Deste modo, manteremos a possibilidade de análise através do cálculo de propriedades para sistemas sem tempo permitindo ao mesmo tempo a verificação do comportamento temporal dos sistemas especificados usando TCTL.

Na rede GHENeSys, tanto os lugares (boxes) quanto as transições (atividades), podem representar subredes através da existência dos elementos "macros". Também temos o fato de que a rede GHENeSys admite multiplicidade de marcas nos lugares o que nos leva à necessidade de diferenciar as marcas dependendo do tempo de criação ou permanência num determinado lugar.

Por esta razão, as marcas precisam do atributo tempo, de vital importância para a determinação das atividades habilitadas e as regras de disparo.

Introduzindo multiplicidade nas marcas será necessário uma definição de multi-set que será usada na representação das marcações da rede GHENeSys.

Definição 29 (Multi-set). Um multi-set b, sobre um conjunto $A$, é uma função de $A$ em $N, b: A \rightarrow N$. Se $a \in A$ então $b(a)$ é o número de vezes que o elemento a aparece no multi-set $b . A_{M S}$ é o conjunto de todos os multi-sets sobre $A$. $O$ multi-set vazio e denotado como $\varnothing$.

A seguir será apresentada a definição da rede GHENeSys que permite a representação de sistemas com tempo não determinístico. 
Definição 30 (GHENeSys). GHENeSys é uma tupla $G=\left(L, A, F, K, \Pi, C_{0}, \tau\right)$ na qual $(L, A, F, K, \Pi)$ representa a estrutura da rede, $C_{0}$ é um conjunto de multisets representando a marcação inicial, e $\tau$ é uma função que mapeia os intervalos de tempo a cada elemento da rede.

- $L=B \cup P$, são os lugares os quais podem ser denominados Boxes e pseudoboxes;

- A são as atividades;

- $F \subseteq(L \times A \rightarrow N) \cup(A \times L \rightarrow N)$ é a relação de fluxo;

- $K: L \rightarrow N^{+}$é a função de capacidade;

- $\Pi:(B \cup A) \rightarrow\{0,1\}$ é a função que identifica os elementos macro;

- $C_{0}=\left\{\left(l, \sigma_{j}\right)\left|l \in L, \sigma_{j} \in R^{+}\right| l \mid \leq K(l)\right\}$ é o conjunto das marcas no estado inicial;

- $\tau:(B \cup A) \longrightarrow\left\{Q^{+}, Q^{+} \cup\{\infty\}\right\}$ é uma função que mapeia os intervalos de tempo a cada elemento da rede.

O conjunto das marcas é composto pelos pares $\left(l, \sigma_{j}\right)$ onde $l \in L$ determina o lugar onde a marca se encontra e $\sigma_{j}$ é o relógio que mede o tempo que a marca permanece neste lugar. Os relógios estão sincronizados com um relógio global e são atualizados cada vez que uma transição acontece. Como podem existir várias marcas num mesmo lugar o conjunto das marcas é na realidade um multi-set.

O mapeamento $\tau$ associa um intervalo de tempo para cada elemento do tipo Box ou atividade. Carece de sentido associar um intervalo de tempo aos pseudo-boxes uma vez que estes são elementos com marcação permanente, isto é, a marcação dos pseudo-boxes não é modificada pela dinâmica da rede ${ }^{1}$. Os intervalos de tempo ligados aos elementos da rede seguem diferentes semânticas dependendo do tipo de elemento. Por exemplo, nos boxes, o intervalo de tempo representa o tempo mínimo ou máximo que uma marca pode permanecer nesse elemento. Nas atividades, o intervalo de tempo possui a mesma semântica que as transições nas RPT de Merlin.

Os intervalos de tempo são formados pelos extremos inferior e superior. Para cada elemento $e \in B \cup A$ da rede, o intervalo de tempo será denominado como $[\downarrow e, \uparrow e] \operatorname{com} \downarrow e \in Q^{+} \mathrm{e} \uparrow e \in Q^{+} \cup\{\infty\}$.

\footnotetext{
${ }^{1}$ Existem casos especiais onde a marcação dos pseudo-boxes é modificada o que será discutido mais adiante.
} 
A relação de fluxo $F$ foi modificada para permitir peso nos arcos, como nas redes lugar/transição e as RPT. O resto dos items da definição mantém o mesmo significado atribuído da definição original em (FOYO, 2001). A figura 4.1 mostra um exemplo de rede GHENeSys.

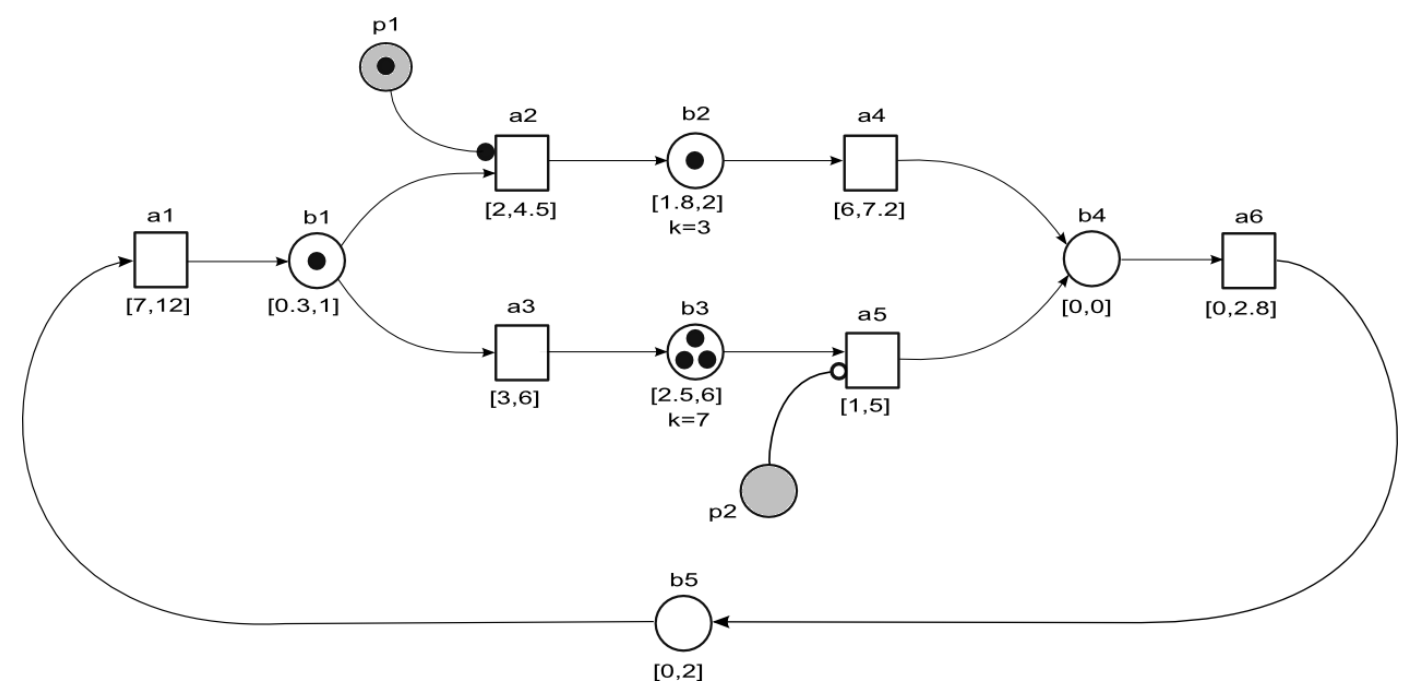

Figura 4.1: Exemplo de rede GHENeSys

Na figura 4.1 os lugares $b 1, b 2, b 3, b 4$ e $b 5$ são boxes enquanto os lugares $p 1$ e $p 2$ são pseudo-boxes. Nos lugares onde a capacidade (denotada pela letra $k$ ) não foram explicitadas possuem capacidade unitária, ou seja, assume-se $k=1$ por defeito.

A modificação feita na definição da rede GHENeSys para a inclusão do tempo modifica as definições prévias de marcação, habilitação, e regras de disparo. Devido a isso, novas definições para estes aspectos devem ser formuladas. A seguir serão tratados os conceitos de estado e transição de estado.

\subsubsection{Estado e transição de estado}

Nas redes de Petri "sem tempo" o estado é representado apenas pela marcação da rede. Já nos sistemas com tempo o estado não só depende da marcação mas também do tempo em que o sistema alcança determinada marcação e do tempo de permanência nesta marcação.

Como se pode ver da definição da rede GHENeSys, as marcas estão associadas não só ao lugar a que pertencem em determinado momento mas também ao tempo que as mesmas permanecem nesse lugar. Portanto, é através da evolução da marcação na rede que é possível determinar o estado geral do sistema que estas representam. 
Como a rede GHENeSys deve ser usada tanto para sistemas sem tempo como para sistemas temporizados, é preciso ter uma definição de marcação que seja complementar.

Definição 31 (Marcação na rede GHENeSys). Uma marcação em G é representada pela distribuição das marcas na rede sem considerar o atributo tempo dessas marcas. Esta marcação é denotada pelo vetor coluna $M$.

$\forall l_{i} \in L, m_{i}=\left(l_{i}, x\right)(C)$ onde $C$ é o multi-set das marcas num determinado momento, e $x$ é um numero real, $n=|L|$ e $i=1 \ldots n$.

Então $M=\left[\begin{array}{lll}m_{1} & \ldots & m_{n}\end{array}\right]^{T}$

Assim o vetor de marcação $M$ está relacionado ao multi-set $C$ o que pode ser expresso como $M=\eta(C)$.

Definição 32 (Estado). Um estado na rede $G$ é um par $s=(M, \sigma)$ onde $M$ é uma marcação atingível em $G$ e $\sigma: A \rightarrow Q^{+}$é um vetor composto por um relógio para cada atividade habilitada pela marcação $M$ disparados no momento em que cada uma delas ficou habilitada pela última vez. Denota-se por $\sigma(a)$ o relógio associado à atividade $a \in A$.

O comportamento da rede é definido por regras de disparo que estabeleçam de forma clara as seqüências de estados admissíveis a partir de um estado inicial. Por seu turno, a regra de disparo depende da semântica de disparo das atividades assim como da semântica da permanência nos boxes.

Quanto a semântica de disparo existem duas possibilidades para determinar a seqüência de disparos em uma RPT. Estas duas semânticas foram identificadas em (RIVIERE et al., 2001) e foram chamadas de semânticas "forte" (strong) e "fraca" (weak). Usando a semântica forte, uma atividade habilitada deve disparar obrigatoriamente se permanece habilitada por um tempo igual ao limite máximo do intervalo ligado à mesma. Na semântica fraca, a atividade não precisa disparar se permanecer habilitada até atingir o limite máximo do intervalo de tempo associado, porém a atividade não poderá mais ser disparada depois deste tempo. Em ambas semânticas uma atividade não pode ser disparada num tempo menor ao limite mínimo do intervalo associado.

A semântica de permanência nos boxes pode ser mínima ou máxima. Isto 
depende da escolha do tempo limite no intervalo ligado a estes elementos:

$$
\forall l_{i} \in L \quad \sigma_{l i}=\left\{\begin{aligned}
\downarrow b & \forall l_{i} \in B \text { para a semântica de permanência mínima } \\
\uparrow b & \forall l_{i} \in B \text { para a semântica de permanência máxima } \\
0 & \forall l_{i} \in P
\end{aligned}\right.
$$

Cada vez que uma marca é gerada através do disparo de uma transição o seu respectivo relógio é atualizado com o tempo de permanência associado ao box onde esta se encontra. Com a passagem do tempo, estes relógios vão se decrementando até serem zerados, o que indica que esta marca pode ser considerada para compor a marcação corrente.

Por exemplo, o conjunto das marcas numa determinada marcação inicial $M_{0}=\left[\begin{array}{llll}m_{01} & m_{02} & \ldots & m_{0 n}\end{array}\right]$ com $n=|L|$ será o seguinte:

$$
C_{0}=m_{01} \times\left(l_{1}, \sigma_{l 1}\right) \cup m_{02} \times\left(l_{2}, \sigma_{l 2}\right) \cup \ldots \cup m_{0 n} \times\left(l_{n}, \sigma_{l n}\right)
$$

onde cada $\sigma_{l i}$ com $i=1 \ldots n$, é inicializado segundo a equação 4.1.

Na rede GHENeSys o padrão é adotar as semânticas forte e mínima.

Seja uma atividade $a_{1}$ com intervalo de tempo $\left[\downarrow a_{1}, \uparrow a_{1}\right]$ habilitada pelo conjunto de marcas $C_{1}$, então $a_{1}$ pode ser disparada em um instante de tempo entre $\left(\sigma+\downarrow a_{1}\right)$ e antes de $\left(\sigma+\uparrow a_{1}\right)$. Quando $a_{1}$ é disparada o conjunto de marcas é modificado para $C_{2}$, os relógios das marcas criadas assumem o valor do tempo mínimo dos lugares em que foram criadas, e os relógios das marcas que já existiam são decrementados no valor de tempo consumido no disparo de $a_{1}$ (que é um valor entre $\downarrow a_{1}$ e $\left.\uparrow a_{1}\right)$. Finalmente, o relógio global então é incrementado com o mesmo valor usado para disparar $a_{1}$. Claramente o limite inferior para os relógios de cada marca é o valor zero.

Isto significa que a marcação $M_{2}=\eta\left(C_{2}\right)$ é atingível a partir de $M_{1}=\eta\left(C_{1}\right)$ pelo disparo de $a_{1}$ em um tempo $\delta\left(\downarrow a_{1} \leq \delta \leq \uparrow a_{1}\right)$. Esta operação corresponde a uma transição de estado e pode ser denotada por $s_{1}\left[a_{1}>s_{2}\right.$, onde $s_{1}=\left(M_{1}, \sigma_{1}\right)$ e $s_{2}=\left(M_{2}, \sigma_{2}\right)$. Por $R\left(s_{0}\right)$ entende-se o conjunto de todos os estados atingíveis a partir de $s_{0}$.

O comportamento da rede GHENeSys estará definido uma vez que se determine todos os cenários de disparo possíveis a partir da marcação inicial, o que leva recursivamente à avaliação das atividades disparáveis em cada estado atingível a partir da marcação inicial.

Genericamente, a definição de transição de estado pode ser resumida intro- 
duzindo o seguinte conjunto de notações e definições:

Definição 33 (Condição de habilitação). Uma atividade a $\in$ A está habilitada no estado $s=(M, \sigma)$ com $s \in R\left(s_{0}\right), M=\eta\left(C_{t}\right)$ e $x \in R^{+}$se as seguintes condições são satisfeitas:

$$
\begin{aligned}
& \forall l_{i} \in \bullet a,\left(l_{i}, x\right)\left(C_{t}\right) \geq n\left[l_{i}, a\right] \\
& \forall l_{i} \in a^{\bullet}, m_{i} \leq K\left(l_{i}\right)-n\left[a, l_{i}\right]
\end{aligned}
$$

onde $n\left[l_{i}, a\right]$ é o peso do arco que liga o lugar $l_{i}$ à atividade a e $n\left[a, l_{i}\right]$ é o peso do arco que liga a atividade a ao lugar $l_{i}$.

As atividades que satisfazem essas condições no estado s são ditas habilitadas em s. O conjunto das atividades habilitadas em $s$ é denotado por enb(s) ou $\operatorname{enb}(M)$.

A condição 4.3 garante a existência de marcas nas pré-condições de $a$ que satisfariam a condição de habilitação num determinado tempo. A condição 4.4 garante a capacidade nas pós-condições de $a$ para admitir estas marcas. Portanto a condição de habilitação independe do tempo.

Assim, a habilitação constitui uma condição necessária para que esta atividade seja disparável. Nesse caso, além da condição de habilitação sem tempo é preciso determinar o tempo mínimo de permanência neste estado.

Definição 34 (Tempo mínimo para habilitação). O tempo mínimo para habilitação é o menor intervalo de tempo necessário para que o conjunto mínimo de marcas que compõem a condição de habilitação tenham seus respectivos relógios zerados.

O tempo mínimo de habilitação num estado deve ser computado para todas as atividades habilitadas no estado, $\nu_{s}: a \rightarrow R^{+}$e compõe um vetor coluna de dimensão $|e n b(s)|$.

Seja um determinado estado $s=(M, \sigma)$ com $s \in R\left(s_{0}\right), M=\eta\left(C_{t}\right)$ e $x \in R^{+}$, e o conjunto de atividades habilitadas enb(s). O tempo mínimo de habilitação pode ser calculado usando o seguinte algoritmo: 


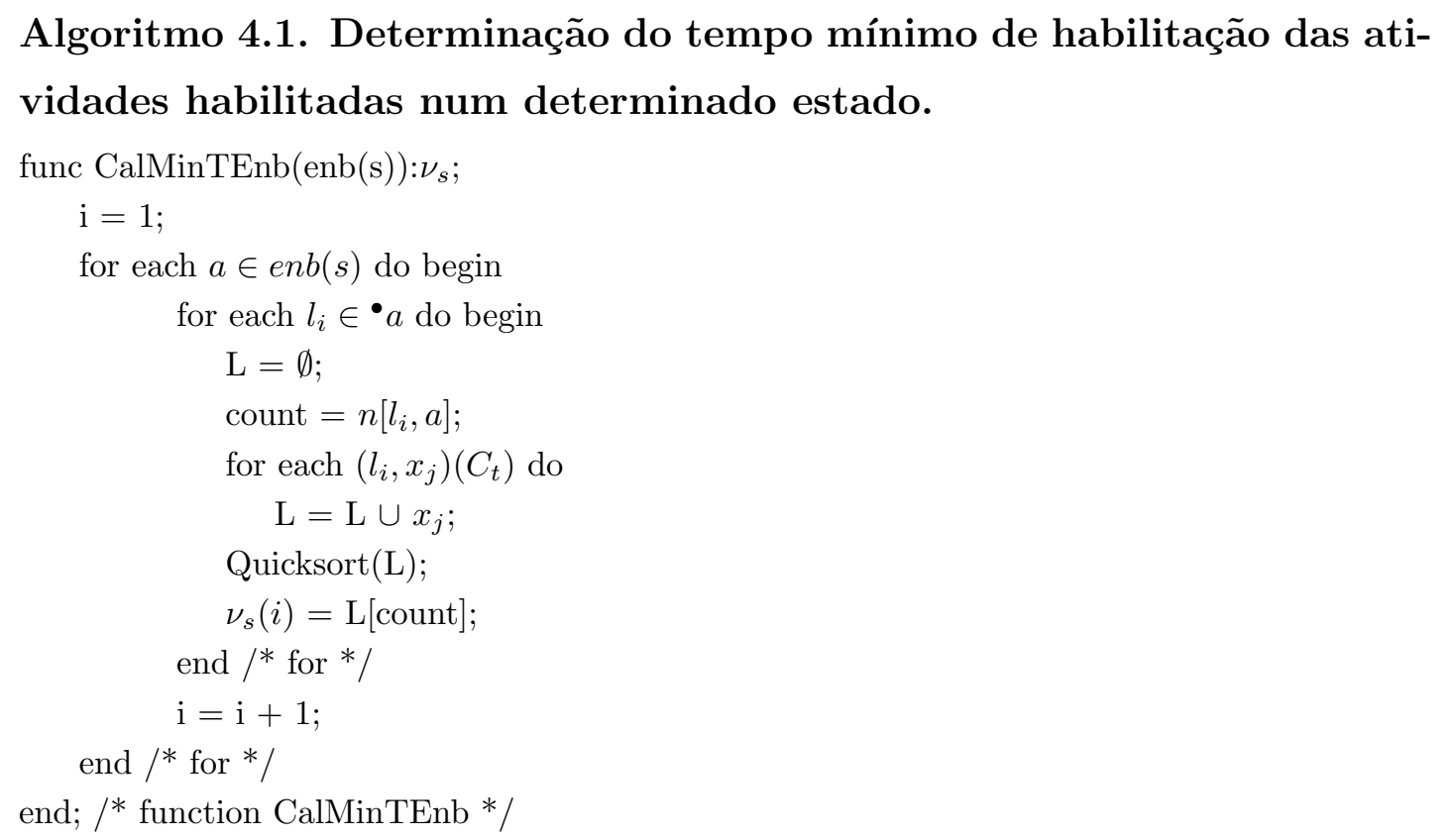

Como nas RPT, uma atividade habilitada só pode disparar se o limite inferior do seu intervalo estático de tempo é menor ou igual aos limites superiores dos intervalos estáticos de tempo das atividades habilitadas restantes.

Definição 35 (Condição para o disparo). Uma atividade a $\in$ A pode ser disparada no estado $s=(M, \sigma)$ se e somente se:

$$
\begin{gathered}
a \in \operatorname{enb}(s) ; \\
\forall a_{i} \in \operatorname{enb}(s), a_{i} \neq a, \downarrow a+\nu_{s}(a)-\sigma(a) \leq \uparrow a_{i}+\nu_{s}\left(a_{i}\right)-\sigma\left(a_{i}\right)
\end{gathered}
$$

O conjunto das atividades disparáveis em s é denotado por $\Upsilon_{s}, \Upsilon_{s} \subseteq$ enb(s).

Para determinar as mudanças provocadas pelo disparo de uma atividade devemos primeiro determinar as atividades habilitadas pela marcação atual assim como os tempos de habilitação para cada uma delas. Estes dados, conjuntamente com os intervalos ligados às atividades permitem determinar as atividades que de fato podem ser disparadas, como colocado na definição 35.

A transição de estados pode então ser definida de forma integrada utilizando os conceitos acima e assumindo a semântica forte.

Definição 36 (Transição de estados). O disparo de uma atividade $a \in \Upsilon_{s}$ leva a uma transição de estado $\left(M_{1}, \sigma_{1}\right) \stackrel{a, \delta}{\longrightarrow}\left(M_{2}, \sigma_{2}\right)$ em um tempo $\delta \in R^{+}$, tal que $\downarrow a+\nu_{s}(a)-\sigma_{1}(a) \leq \delta \leq \uparrow a_{i}+\nu_{s}\left(a_{i}\right)-\sigma_{1}\left(a_{i}\right), \forall a_{i} \in e n b\left(s_{1}\right)$, provocando as 
seguintes mudanças:

$$
\begin{gathered}
\forall\left(l_{i}, x_{j}\right) \in C_{t 1}, C_{t 1}=C_{t 1} \backslash\left\{\left(l_{i}, x_{j}\right)\right\} \cup\left\{\left(l_{i}, \max \left(0, x_{j}-\delta\right)\right)\right\} ; \\
\forall l_{i} \in \bullet^{\bullet} \cap B, C_{t 2}=C_{t 1} \backslash n\left[l_{i}, a\right] \times\left\{\left(l_{i}, 0\right)\right\} ; \\
\forall l_{i} \in a^{\bullet} \cap B, C_{t 2}=C_{t 1} \cup n\left[a, l_{i}\right] \times\left\{\left(l_{i}, \downarrow l_{i}\right)\right\} ; \\
M_{2}=\eta\left(C_{t 2}\right) ; \\
\forall a_{k} \in \operatorname{enb}\left(s_{2}\right), \sigma_{2}\left(a_{k}\right)=0 ; \\
\forall a_{k} \in \operatorname{enb}\left(s_{1}\right) \cap \operatorname{enb}\left(s_{2}\right), a_{k} \neq a, \sigma_{2}\left(a_{k}\right)=\sigma_{1}+\delta-\nu_{s}\left(a_{k}\right) ; \\
\sigma=\sigma+\delta ;
\end{gathered}
$$

$s \stackrel{a, \delta}{\longrightarrow} s^{\prime}$ define o conjunto de estados $s^{\prime}$ atingíveis a partir do estado s disparando a transição a num tempo $\delta$, ou seja, $\left|s^{\prime}\right|=\left|\Upsilon_{s}\right|$.

A definição acima também pode ser interpretada como um procedimento para determinar o comportamento de uma RPT onde o primeiro passo é reajustar os tempos nas marcas decrementando os respectivos relógios. Em seguida, as marcas das pré-condições da atividade disparada são removidas - cujos relógios estavam zerados. No próximo passo as novas marcas são criadas nas pós-condições e os relógios dessas marcas atualizados com o valor do tempo de permanência no lugar onde foram criadas. Nesse ponto, o conjunto das marcas já foi devidamente construído e portanto a nova marcação pode ser determinada. Com a nova marcação é possível corrigir o tempo das atividades em conflito com a atividade disparada, zerando os relógios dessas atividades. Para as demais atividades o relógio é atualizado incrementando do valor $\delta$ o tempo que os mesmas tinham no estado recém abandonado. Finalmente o relógio global é atualizado usando o seu valor anterior e o tempo transcorrido no disparo.

Na rede apresentada na figura 4.1 o estado inicial é determinado pelo seguinte conjunto de marcas:

$$
C_{0}=\{(1,0.3),(2,1.8),(3,2.5),(3,2.5),(3,2.5),(6,0)\}
$$

Note que os lugares são numerados seguindo uma ordem determinada, primeiro os boxes e logo os pseudo-boxes. Neste caso o elemento $(6,0)$ corresponde à marca no pseudo-box $p 1$.

Para determinar as atividades habilitadas na marcação inicial $M_{0}=\eta\left(C_{0}\right)$ é possível usar a representação matricial original da rede como sugerida em (FOYO, 2001) uma vez que a condição de habilitação não depende do tempo. Em seguida 
deve-se calcular o tempo mínimo de habilitação para cada uma das atividades habilitadas. Os resultados obtidos são mostrados a seguir:

$\left.\begin{array}{ccccc}\text { enb }\left(s_{0}\right)= & a_{2} & a_{3} & a_{4} & a_{5} \\ \nu_{s_{0}}= & 0.3 & 0.3 & 1.8 & 2.5 \\ I\left(\Upsilon_{s_{0}}\right) & {\left[\begin{array}{ll}2.3 & 4.8\end{array}\right]} & {\left[\begin{array}{lll}3.3 & 4.8\end{array}\right]} & -\end{array}\right]$

A última fila mostra os intervalos válidos de $\delta$ para cada uma das transições segundo a definição 36. Claramente a atividade $a_{4}$ não é disparável no estado $s_{0}$ segundo a definição 35, portanto não foi inserida no vetor $I$.

Se a atividade $a_{2}$ é disparada com $\delta=2.4$ no estado $s_{0}=\left(M_{0}, \sigma_{0}\right)$. Como

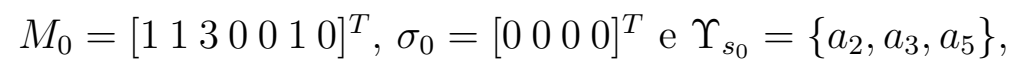

$$
\left(M_{0}, \sigma_{0}\right) \stackrel{a_{2}, 2.4}{\longrightarrow}\left(M_{1}, \sigma_{1}\right) \text {. }
$$

Segundo a definição 36 isto levaria ao estado $s_{1}=\left(M_{1}, \sigma_{1}\right) \operatorname{com} M_{1}=$

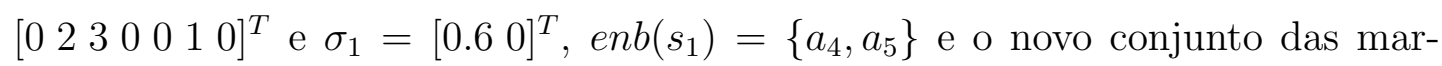
cas seria o seguinte:

$$
C_{1}=\{(2,0),(2,1.8),(3,0.1),(3,0.1),(3,0.1),(6,0)\}
$$

Note-se que a atividade $a_{4}$ está a 0.6 unidades de tempo habilitada no estado $s_{1}$, pois precisou de 1.8 unidades de tempo para zerar a marca na sua pré-condição. As atividades $a_{2}$ e $a_{3}$ não estão mais habilitadas em $s_{1}$, portanto não aparecem em $\sigma_{1}$. O tempo $\delta$ transcorrido não foi suficiente para zerar as marcas em $b_{3}$, portanto $\nu_{s 1}\left(a_{5}\right)=0.1$ e $\sigma_{1}\left(a_{5}\right)=0$.

\subsubsection{A abordagem de classes de estados em GHENeSys}

Na rede GHENeSys o tempo é modelado usando números reais (ou racionais), portanto, existirá um número infinito de estados concretos qualquer que seja a estrutura da rede. A abordagem das classes de estados é aplicável às redes GHENeSys, agrupando estados concretos em classes de estados obtendo um número finito de estados, uma vez que esta rede é em princípio limitada. Portanto, os problemas de atingibilidade (reachability) e limitação (boundedness), que são indecidíveis nas RPT (JONES; LANDWEBER; LIEN, 1977), de fato não se aplicam às redes GHENeSys.

Mesmo que existam boxes com capacidade infinita existem condições suficientes para determinar limitação (boundedness) - que podem ser usadas conjuntamente com os algoritmos de geração das classes de estado, de modo que a 
terminação do algoritmo seja garantida - mesmo para redes com número infinito de estados.

As classes de estados são determinadas por um par $s=(M, \Delta)$ onde $M$ é a marcação comum a todos os estados e $\Delta$ representa o domínio temporal. O domínio temporal é uma fórmula caracterizada pela união de todos os tempos de disparo associados aos estados pertencentes à classe de estado, ou seja:

$$
\Delta=\left(\bigwedge_{a \in e n b(s)} \operatorname{tmin}(a) \leq \delta \leq \operatorname{tmax}(a)\right)
$$

O domínio temporal é representado usando DBM's de ordem $|e n b(s) \cup\{o\}|^{2}$. O intervalo de tempo válido para cada atividade no domínio temporal é determinado da seguinte forma:

$$
\forall a, a_{k} \in \Upsilon_{s} \quad, \quad \operatorname{tmin}(a) \leq \delta(a) \leq \min \left(\max \left(a_{k}\right)\right)
$$

Note-se que, dependendo dos valores de $\operatorname{tmin}(a)$ e $\operatorname{tmax}(a)$ em 4.6, para cada atividade $a$ podem existir atividades habilitadas não disparáveis. Os valores admissíveis para o tempo válido de cada atividade refere-se apenas às atividades disparáveis em $s$, ou seja, é denotado como $I(a), a \in \Upsilon_{s}$. Este intervalo $I$ é representado por uma matriz de $n \times 2$ onde $n$ é o número de atividades disparáveis em $s, n=\left|\Upsilon_{s}\right|$.

Mesmo assim, no domínio temporal devem estar representadas as atividades habilitadas pois o tempo em que cada atividade permanece habilitada conta para a determinação dos próximos estados ou classes de estados.

Partindo de um estado inicial, onde os relógios das atividades estão todos zerados determinam-se as classes de estados usando a definição 36. Para isto, é preciso conhecer o marcação dos pseudo-boxes. A marcação destes elementos, considerarados elementos externos ao sistema, é definida pela marcação inicial e está associada a diferentes cenários quanto aos sinais externos. No caso destes elementos não serem externos sua marcação é determinada pelo elemento ao qual estão ligados através da propriedade MasterElement.

A exemplo das RPT, o objetivo final é, usando a abordagem das classes de estados, construir grafos que mostram como se preservam várias propriedades, como foi discutido na capítulo anterior, com a diferença de que, no caso da rede GHENeSys, as marcas possuem tempos associados. Os tempos mínimos de habilitação para cada atividade são representados também por inequações.

\footnotetext{
${ }^{2} \mathrm{~A}$ variável $o$ representa o valor zero.
} 
Os tempos mínimos de habilitação (menores e maiores) - denotados pelos símbolos $\downarrow \nu_{s}$ e $\uparrow \nu_{s}$ respectivamente - serão representados por uma matriz de ordem $n \times 2$ onde $n$ é o número de atividades habilitadas no estado $s$. Obviamente estes valores afetarão os intervalos de tempo de cada transição entre as classes de estados.

Dada a transição $s \stackrel{a}{\rightarrow} s^{\prime}$ estes tempos são determinados da seguinte forma:

$$
\begin{aligned}
& \forall\left(l_{i}, x_{j}\right) \in C_{s}, C_{s}=C_{s} \backslash\left\{\left(l_{i}, x_{j}\right)\right\} \cup\left\{\left(l_{i}, \max \left(0, x_{j}-\min \left(I_{\delta_{a}}\right)\right)\right)\right\} ; \\
& \forall l_{i} \in \bullet \cap B, C_{s^{\prime}}=C_{s} \backslash n\left[l_{i}, a\right] \times\left\{\left(l_{i}, 0\right)\right\} ; \\
& \forall l_{i} \in a^{\bullet} \cap B, C_{s^{\prime}}=C_{s} \cup n\left[a, l_{i}\right] \times\left\{\left(l_{i}, \downarrow l_{i}\right)\right\} ; \\
& \forall a_{k} \in \operatorname{enb}(M), \text { Calcular } \nu_{s^{\prime}}\left(a_{k}\right) \text { usando o algoritmo } 4.1 ; \\
& \forall a_{k} \in \operatorname{enb}(M), \uparrow \nu_{s^{\prime}}\left(a_{k}\right)=\nu_{s^{\prime}}\left(a_{k}\right) ; \\
& \forall a_{j} \in \operatorname{new}(M), \downarrow \nu_{s^{\prime}}\left(a_{j}\right)=\nu_{s^{\prime}}\left(a_{j}\right) ; \\
& \forall a_{l} \in\{\operatorname{enb}(M) \backslash \text { new }(M)\}, \downarrow \nu_{s^{\prime}}\left(a_{l}\right)=\max \left(0, \nu_{s^{\prime}}\left(a_{l}\right)+\min (\operatorname{Ia})-\max (\operatorname{Ia})\right) ; \\
& \text { onde new }(M) \text { é o conjunto de atividades recém habilitadas. }
\end{aligned}
$$

Como na rede GHENeSys é atribuído um intervalo de tempo (de permanência) aos lugares, onde as marcas carregam o relógio que conta este tempo, será também necessário rever a definição de atividades habilitadas.

Definição 37 (Atividades recém habilitadas). Seja uma transição de estado s $\stackrel{a, I(a)}{\longrightarrow} s^{\prime}$, admissivel em uma rede $\boldsymbol{G H E N e S y s ~} G$. As atividades recém habilitadas no estado s' são aquelas que estavam habilitadas em s mas não eram disparáveis (desde que independentes das disparáveis) unido ao conjunto das novas atividades habilitadas no novo estado, ou seja:

$$
n e w\left(M^{\prime}\right)=\left(\operatorname{enb}\left(M^{\prime}\right) \cap \operatorname{enb}\left(M+D \Lambda^{+} \vec{a}\right)\right) \backslash\{\operatorname{enb}(M) \backslash\{a\}\}
$$

onde D é a matriz de correção da marcação permanente, $\Lambda^{+}$é a matriz de incidência formada só pelos coeficientes positivos, e $\vec{a}$ é o vetor de disparo da atividade a. O conjunto das atividades recém habilitadas em $s^{\prime}$ é denotado por new $\left(s^{\prime}\right)$ ou new $\left(M^{\prime}\right)$.

O comportamento da rede GHENeSys é caracterizado pelo grafo das classes de estados definido da seguinte forma: 
Definição 38 (Grafo das classes de estados). O grafo das classes de estados de uma rede $\boldsymbol{G H E N e S y s} G=\left(L, A, F, K, \Pi, C_{0}, \tau\right)$ é a estrutura:

$$
S G=\left(\Omega, \stackrel{*}{\longrightarrow}, C_{\epsilon}\right)
$$

onde $\Omega$ é o conjunto de todas as classes de estados, $\stackrel{*}{\longrightarrow}$ representa todas as transições de estados possíveis a partir da classe de estado inicial, e $C_{\epsilon}$ que é determinada pela marcação no estado inicial e pelo domínio temporal $\Delta_{0}$ definido por:

$$
\Delta_{0}=\left\{\begin{array}{rl}
\bigwedge_{a \in \operatorname{enb}(s)} & a \leq \operatorname{tmax}(a) \wedge-a \leq-\operatorname{tmin}(a) \\
\bigwedge_{\left(a, a^{\prime}\right) \in \operatorname{enb}(s)^{2}} & a-a^{\prime} \leq \operatorname{tmax}(a)-\operatorname{tmin}\left(a^{\prime}\right)
\end{array}\right.
$$

Note que $\Delta_{0}$ já está na forma canônica. Como foi mostrado em (BOUCHENEB; MULLINS, 2003) é possível determinar o novo domínio temporal a partir do domínio temporal anterior na sua forma canônica com uma complexidade $O\left(n^{2}\right)$ onde $n$ é o número de atividades habilitadas no estado.

A forma canônica do domínio temporal $\Delta$ é definida pela seguinte matriz de ordem $|\operatorname{enb}(s) \cup\{o\}|$ :

$$
\forall x, y \in \operatorname{enb}(s) \cup\{o\})^{2}, \Delta_{x y}=\operatorname{Sup}_{\Delta}(x-y)
$$

onde $\operatorname{Sup}_{\Delta}(x-y)$ é o supremo ${ }^{3}$ de $x-y$ no domínio de $\Delta$.

O algoritmo para determinar o novo domínio temporal na sua forma canônica é baseado na seguinte proposição:

Proposição 39 (Determinação das classes sucessoras). Seja $s^{\prime}=(M, \Delta)$ uma classe de estado na sua forma canônica obtida através do disparo de uma atividade $a_{i}$ com intervalo de tempo associado $I\left(a_{i}\right), s \stackrel{a_{i}, I\left(a_{i}\right)}{\longrightarrow} s^{\prime}$, e $a_{j}$ uma atividade habilitada pela marcação $M$.

- $a_{j}$ é disparável em s', ou seja, $a_{j} \in \Upsilon_{s^{\prime}}$ em um intervalo $I\left(a_{j}\right)$.

- $\uparrow \nu_{s^{\prime}} e \downarrow \nu_{s^{\prime}}$ são o maior e o menor tempo de habilitação da atividade $a_{j}$.

- a classe sucessora de $s^{\prime}, s^{\prime} \stackrel{a_{j}, I\left(a_{j}\right)}{\longrightarrow} s^{\prime \prime}, s^{\prime \prime}=\left(M^{\prime}, \Delta^{\prime}\right)$ é computada como:

\footnotetext{
${ }^{3}$ Se o domínio $\Delta$ é representado pelo grafo direcionado com pesos nos arcos onde a cada variável corresponde um nó e o peso do arco entre dois nós é a diferença entre essas variáveis, o supremo de um par de nós equivale ao caminho mais curto entre esses nós
} 


$$
\begin{gathered}
\forall a^{\prime} \in \operatorname{enb}\left(M^{\prime}\right), \quad \Delta_{a^{\prime} o}^{\prime}=\left\{\begin{array}{r}
\uparrow a^{\prime}+\uparrow \nu_{s^{\prime}}\left(a^{\prime}\right) \text { se } a^{\prime} \in \text { new }\left(M^{\prime}\right) ; \\
\Delta_{a^{\prime} a} \text { nos casos restantes }
\end{array}\right. \\
\Delta_{o a^{\prime}}^{\prime}=\left\{\begin{array}{r}
-\left(\downarrow a^{\prime}+\downarrow \nu_{s^{\prime}}\left(a^{\prime}\right)\right) \text { se } a^{\prime} \in \text { new }\left(M^{\prime}\right) ; \\
M i n_{u \in e n b(M)} \Delta_{u a^{\prime}} \text { nos casos restantes }
\end{array}\right. \\
\forall\left(a, a^{\prime}\right) \in \operatorname{enb}\left(M^{\prime}\right)^{2}, \Delta_{a a^{\prime}}^{\prime}=\left\{\begin{array}{r}
0 \text { se } a=a^{\prime} ; \\
\Delta_{a o}^{\prime}+\Delta_{o a^{\prime}}^{\prime} \text { se } a \vee a^{\prime} \in \text { new }\left(M^{\prime}\right) ; \\
\operatorname{Min}\left(\Delta_{a a^{\prime}}, \Delta_{a o}^{\prime}+\Delta_{o a^{\prime}}^{\prime}\right) \text { nos casos restantes }
\end{array}\right.
\end{gathered}
$$

A prova da proposição 39 é mostrada no apêndice B.2.

O algoritmo para o cálculo do grafo das classes de estados usa a definição 36 na determinação das classes de estados sucessoras. Uma determinada classe de estado $C_{w}=\left(M_{w}, \Delta_{w}\right)$ tem uma classe de estado sucessora se existe ao menos uma atividade disparável em $C_{w}$, ou seja, $\Upsilon_{s_{w}} \neq \emptyset$. Para as classes de estados lineares, podemos afirmar que existe uma classe de estado sucessora para cada atividade disparável nessa classe de estado.

Para determinar as atividades disparáveis, assim como os intervalos válidos de disparo de cada uma delas, usamos um algoritmo baseado na seguinte proposição:

Proposição 40 (Intervalos válidos de disparo). Seja $s^{\prime}=\left(M^{\prime}, \Delta^{\prime}\right)$ uma classe de estado na sua forma canônica obtida através do disparo de uma classe $s=(M, \Delta)$, os intervalos válidos de disparo para cada atividade habilitada em ${ }^{\prime}$, $a^{\prime} \in \operatorname{enb}\left(s^{\prime}\right)$ são determinados da seguinte forma:

$$
\begin{array}{r}
\delta_{\min }\left(a^{\prime}\right)=-\Delta_{o a^{\prime}}^{\prime} \quad a^{\prime} \in \operatorname{enb}\left(M^{\prime}\right) ; \\
\delta_{\max }=\min \left(\Delta_{a^{\prime} o}^{\prime}\right) \\
I\left(a^{\prime}\right)=\left[\delta_{\min }\left(a^{\prime}\right), \delta_{\max }\right] ; \quad \operatorname{enb}\left(M^{\prime}\right) ;
\end{array}
$$

Note que $a^{\prime} \in \Upsilon_{s^{\prime}}$ sse $\Delta_{a a^{\prime}}^{\prime} \geq 0 \forall a \in \operatorname{enb}\left(s^{\prime}\right)$.

As classes de estados sucessoras podem ser calculadas usando o seguinte algoritmo:

- Determinar as atividades habilitadas pela marcação $M_{w}$, enb $\left(M_{w}\right)$;

- Determinar o menor e o maior tempo de habilitação para cada atividade recém habilitada;

- Determinar as atividades disparáveis em $C_{w}, \Upsilon_{s_{w}}$; 
- Determinar o intervalo de disparo válido para cada atividade disparável em $C_{w}$, ou seja, $I\left(a_{j}\right), \forall a_{j} \in \Upsilon_{s_{w}}$;

- Para cada atividade disparável em $C_{w}, a_{j} \in \Upsilon_{s_{w}}$, calcular a classe sucessora:

1. Determinar a nova marcação $M_{w^{\prime}}$.

2. Determinar o novo domínio temporal $\Delta_{w^{\prime}}$ na sua forma canônica.

3. Comparar se existe alguma classe de estado equivalente e, se existir, eliminar a classe recém criada e apontar a transição de estado recém computada para a classe de estado equivalente.

4. Repetir estes passos até ter processado todas as atividades disparáveis em $C_{w}$.

Este algoritmo é executado até que todas as classes sucessoras tenham sido computadas. A garantia de terminação está ligado ao fato da rede subjacente ser limitada, o que pode ser previamente determinado.

Usando o exemplo da figura 4.1, pode-se notar que os pseudo-boxes mantém uma marcação persistente. Sendo assim, teremos a seguinte classe de estado inicial:

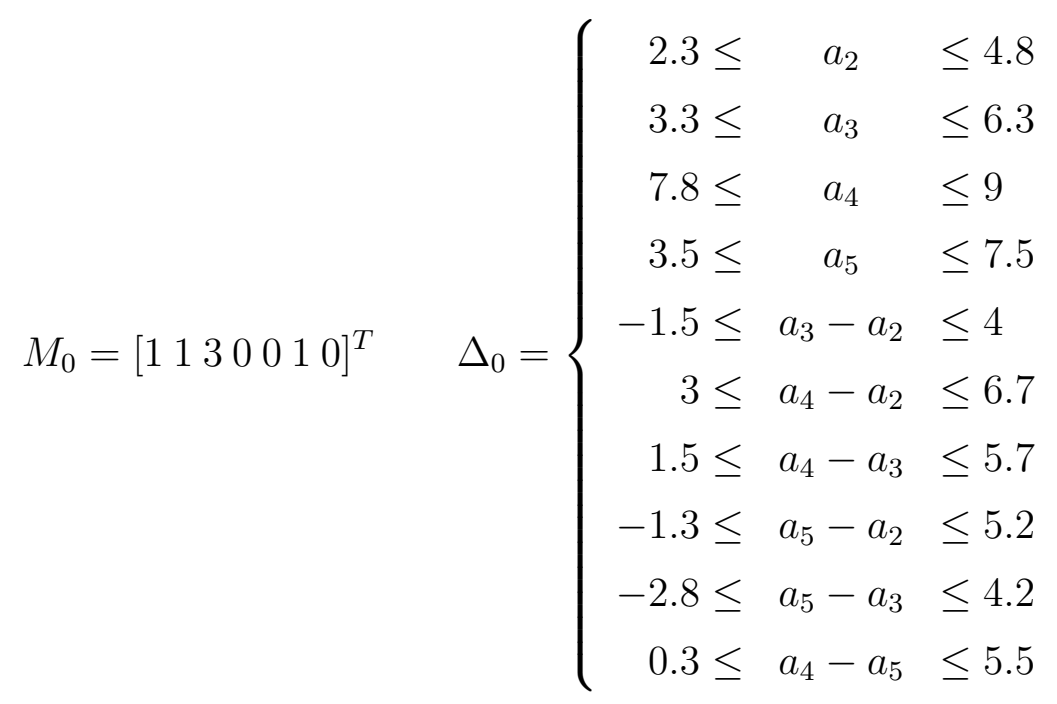

Disparando $a_{2}$ em um tempo $\sigma \in I\left(a_{2}\right)=[2.3,4.8]$ leva à classe de estado $C_{1}=\left(M_{1}, \Delta_{1}\right)$ :

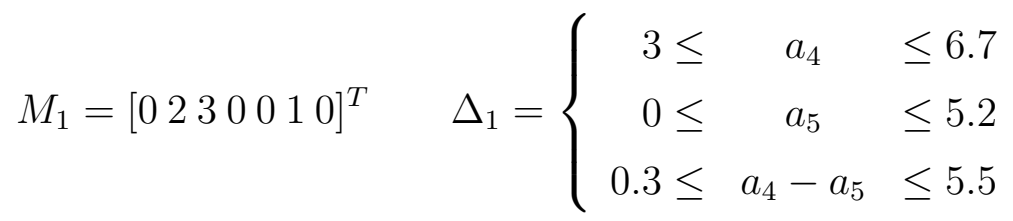

disparando $a_{3}$ em um tempo $\sigma \in I\left(a_{3}\right)=[3.3,4.8]$ leva à classe de estado $C_{2}=$ 
$\left(M_{2}, \Delta_{2}\right)$ :

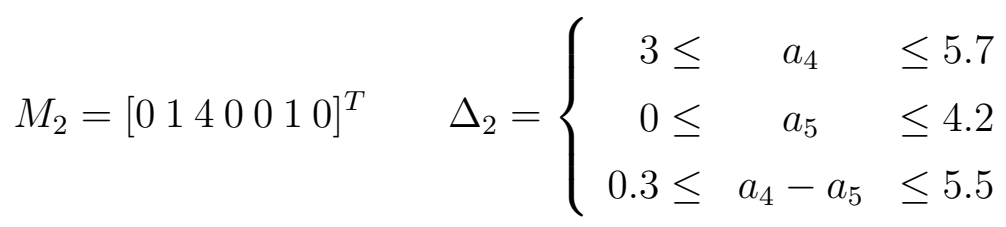

e disparando $a_{5}$ em um tempo $\sigma \in I\left(a_{5}\right)=[3.5,4.8]$ leva à classe de estado $C_{3}=\left(M_{3}, \Delta_{3}\right)$ :

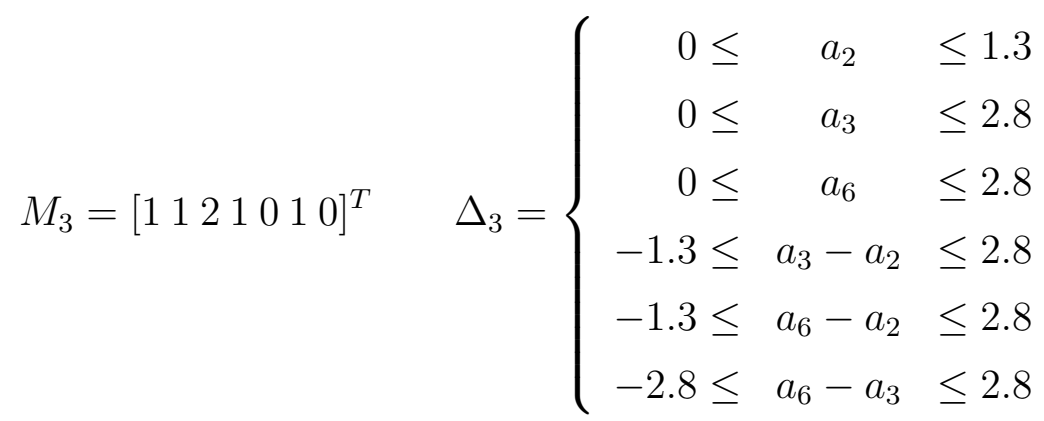

\subsubsection{As classes de estados com restrições na rede GHENeSys}

No capítulo anterior foi mostrado como computar as classes com restrições nas RPT. Existem duas discrepâncias entre este processo e o da rede GHENeSys: primeira é a introdução do tempo nas marcas, lembrando que estes tempos são atualizados dependendo do lugar onde a marca é criada; a segunda refere-se à manipulação dos pseudo-boxes.

O primeiro caso, tem como impacto apenas um incremento de tempo nas atividades recém habilitadas devido ao acréscimo do tempo mínimo de habilitação para cada atividade recém habilitada.

No segundo caso, como os pseudo-boxes podem assumir marcação externa (ou de um outro elemento da rede), e portanto cada vez que se determinam as atividades habilitadas é preciso executar um método que atualiza a marcação dos pseudo-boxes.

Para a construção do GTT, é necessário construir antes o CSCG como foi mostrado no capítulo anterior. O algoritmo é formado pelas seguintes etapas:

- Determinar as atividades habilitadas pela marcação $M_{w}$, enb $\left(M_{w}\right)$;

- Determinar o menor e o maior tempo de habilitação para cada atividade recém habilitada;

- Ajustar os valores de EFT' e LFT' de cada atividade recém habilitada 
usando os respectivos tempos de habilitação:

$$
\begin{aligned}
\forall a \in \operatorname{new}\left(M_{w}\right) \quad & \operatorname{EFT}^{\prime}(a)=\operatorname{EFT}(a)+\downarrow \nu_{s_{w}}(a) \\
\operatorname{LFT}^{\prime}(a) & =\operatorname{LFT}(a)+\uparrow \nu_{s_{w}}(a)
\end{aligned}
$$

- Determinar as atividades disparáveis em $C_{w}, \Upsilon_{s_{w}}$;

- Determinar o intervalo de disparo válido para cada atividade disparável em $C_{w}$, ou seja, $I\left(a_{j}\right), \forall a_{j} \in \Upsilon_{s_{w}}$;

- Para cada atividade disparável em $C_{w}, a_{j} \in \Upsilon_{s_{w}}$ calcular a classe sucessora:

1. Determinar a nova marcação $M_{w^{\prime}}$.

2. Determinar o novo domínio temporal $\Delta_{w^{\prime}}$ na sua forma canônica.

3. Verificar se a nova classe é estável.

4. Se não for estável, dividi-la em duas classes estáveis.

5. Comparar se existe alguma classe de estado equivalente e, se existir, eliminar a classe recém criada e apontar a transição de estado recém computada para a classe de estado equivalente.

6. Repetir estes passos até ter processado todas as atividades disparáveis em $C_{w}$.

O algoritmo para o cálculo do CSCG de uma rede GHENeSys é baseado nas seguintes proposições.

\section{Proposição 41 (Determinação do domínio para uma classe sucessora).}

Seja $s=(M, \Delta)$ uma classe de estado na sua forma canônica e $a_{j}$ uma atividade habilitada pela marcação $M$.

- $a_{j}$ é disparável em sse $E F T^{\prime}\left(a_{j}\right)-\Delta_{a_{j} o} \leq \operatorname{Min}_{u \in e n b(M)}\left(\operatorname{LFT}^{\prime}(u)-\Delta_{o u}\right)$.

- se $a_{j}$ é disparável em s então o intervalo de disparo é: $\left.I\left(a_{j}\right)=\left[\max \left(0, \operatorname{EFT}^{\prime}\left(a_{j}\right)\right)-\Delta_{a_{j} o}\right), \operatorname{Min}_{u \in e n b(M)}\left(\operatorname{LFT}(u)-\Delta_{o u}\right)\right]$.

- o domínio temporal da classe sucessora de s disparando $a_{j}$ no intervalo de disparo $I\left(a_{j}\right), s \stackrel{a_{j} I\left(a_{j}\right)}{\longrightarrow} s^{\prime}, s^{\prime}=\left(M^{\prime}, \Delta^{\prime}\right)$ é computada como: 


$$
\begin{aligned}
& \forall a \in \operatorname{enb}\left(M^{\prime}\right), \Delta_{t o}^{\prime}=\left\{\begin{array}{r}
0 \text { se } a \in \text { new }\left(M^{\prime}\right) ; \\
\operatorname{Min}_{u \in \operatorname{enb}(M)}\left(L F T^{\prime}(u)+\Delta_{a u}\right) \text { nos casos restantes }
\end{array}\right. \\
& \Delta_{o a}^{\prime}=\left\{\begin{array}{r}
\text { se } a \in \text { new }\left(M^{\prime}\right) ; \\
\max \left(0, E F T^{\prime}\left(a_{j}\right)-\Delta_{a_{j} a}\right) \text { nos casos restantes }
\end{array}\right. \\
& \forall\left(a, a^{\prime}\right) \in \operatorname{enb}\left(M^{\prime}\right)^{2}, \Delta_{a a^{\prime}}^{\prime}=\left\{\begin{array}{r}
0 \text { se } a=a^{\prime} \text { ou } a \wedge a^{\prime} \in \text { new }\left(M^{\prime}\right) ; \\
\Delta_{a o}^{\prime}-\Delta_{o a^{\prime}}^{\prime} \text { se } a \vee a^{\prime} \in \text { new }\left(M^{\prime}\right) ; \\
\Delta_{a a^{\prime}} \text { nos casos restantes }
\end{array}\right.
\end{aligned}
$$

Proposição 42 (Identificação de classes não estáveis). Seja $s_{i}=\left(M^{\prime}, \Delta^{\prime}\right)$ uma classe de estados tal que $s_{i-1} \stackrel{I\left(a_{j}\right)}{\rightarrow} s_{i}, s_{i}$ é instável sse satisfaz a:

$\exists a, a^{\prime} \in \operatorname{enb}\left(M^{\prime}\right), a \in$ new $\left(M^{\prime}\right)$ tal que :

$$
\begin{aligned}
& \Delta_{o a^{\prime}}^{\prime}<E F T^{\prime}\left(a^{\prime}\right)-\operatorname{LFT}^{\prime}(a)<\Delta_{a^{\prime} o}^{\prime} \\
& \Delta_{o a^{\prime}}^{\prime}<\operatorname{LFT}^{\prime}\left(a^{\prime}\right)-E F T^{\prime}(a)<\Delta_{a^{\prime} o}^{\prime}
\end{aligned}
$$

Se a condição 4.10 é satisfeita, a' pode ou não ser disparável, dependendo do tempo em que esta foi recém habilitada em uma classe antecessora a $s_{i}$. Se a condição 4.11 é satisfeita a pode ou não ser disparável, dependendo do tempo em que a atividade $a^{\prime}$ foi recém habilitada em uma classe antecessora a $s_{i}$.

As tabelas 4.1 e 4.2 mostram os valores usados na divisão das classes não estáveis.

\begin{tabular}{|l|c|c|}
\hline Condição 4.10 & \multicolumn{2}{|c|}{$\sigma=s_{k} \rightarrow \ldots \rightarrow s_{i-1} \stackrel{a_{j}}{\rightarrow} s_{i}$} \\
\hline Restrição & $s_{i}-s_{k} \geq E F T^{\prime}\left(a^{\prime}\right)-L F T^{\prime}(a)$ & $s_{i}-s_{k}<E F T^{\prime}\left(a^{\prime}\right)-L F T^{\prime}(a)$ \\
\hline$\left[\Delta_{\text {min }}, \Delta_{\text {max }}\right]$ & $E F T^{\prime}\left(a^{\prime}\right)-L F T^{\prime}(a)-\Delta_{o a^{\prime}}^{\prime}$ & $E F T^{\prime}\left(a^{\prime}\right)-\Delta_{a^{\prime} o}^{\prime}-L F T^{\prime}(a)-\epsilon$ \\
\hline$s_{i-1} \stackrel{I\left(a_{j}\right)}{\rightarrow} s_{i a}$ & $E F T^{\prime}\left(a_{j}\right)-\Delta_{a_{j} o}$ & $\operatorname{Min}_{u \in e n b(M)}\left(L F T(u)-\Delta_{o u}\right)$ \\
\hline$s_{i-1} \stackrel{I\left(a_{j}\right)}{\rightarrow} s_{i b}$ & $E F T^{\prime}\left(a_{j}\right)-\Delta_{a_{j} o}$ & $\operatorname{Min}_{u \in e n b(M)}\left(L F T(u)-\Delta_{o u}\right)$ \\
\hline
\end{tabular}

Tabela 4.1: Restrição usada na divisão da classe $s_{i}$ para a condição 4.10

\begin{tabular}{|l|c|c|}
\hline Condição 4.11 & \multicolumn{2}{|c|}{$\sigma=s_{k} \rightarrow \ldots \rightarrow s_{i-1} \stackrel{a_{j}}{\rightarrow} s_{i}$} \\
\hline Restrição & $s_{i}-s_{k}>L F T^{\prime}\left(a^{\prime}\right)-E F T^{\prime}(a)$ & $s_{i}-s_{k} \leq L F T^{\prime}\left(a^{\prime}\right)-E F T^{\prime}(a)$ \\
\hline$\left[\Delta_{\min }, \Delta_{\max }\right]$ & $L F T^{\prime}\left(a^{\prime}\right)-E F T^{\prime}(a)-\Delta_{o a^{\prime}}^{\prime}+\epsilon$ & $L F T^{\prime}\left(a^{\prime}\right)-\Delta_{a^{\prime}}^{\prime}-E F T^{\prime}(a)$ \\
\hline$s_{i-1} \stackrel{\left(\left(a_{j}\right)\right.}{\longrightarrow} s_{i a}$ & $\Delta_{o a^{\prime}}^{\prime}-\Delta_{o a^{\prime}}+\Delta_{\min }$ & $\operatorname{Min}_{u \in e n b(M)}\left(L F T(u)-\Delta_{o u}\right)$ \\
\hline$s_{i-1} \stackrel{I\left(a_{j}\right)}{\longrightarrow} s_{i b}$ & $\operatorname{Max}\left(0, E F T^{\prime}\left(a_{j}\right)-\Delta_{a_{j} o}\right)$ & $\Delta_{a^{\prime} o}^{\prime}-\Delta_{o a^{\prime}}+\Delta_{\max }$ \\
\hline
\end{tabular}

Tabela 4.2: Restrição usada na divisão da classe $s_{i}$ para a condição 4.11 
Os domínios das novas classes $s_{i a}$ e $s_{i b}$ e o incremento de tempo global são calculados utilizando as proposições 11 e 12 apresentadas no capítulo anterior.

Todos estes algoritmos foram implementados e fazem parte do verificador de modelos que será apresentado no capítulo seguinte.

\subsection{Abordagem modular do processo de veri- ficação em GHENeSys}

Valette (1979) propôs uma definição de hierarquia que pode ser usada no desenvolvimento de redes de Petri através de refinamentos sucessivos. Esta definição era baseada na substituição de transições por subredes que começam e terminam com transições. Foi provado que, se estas subredes constituem "blocos bem formados" certas propriedades como limitação e vivacidade podem ser analisadas nos níveis mais abstratos, com a garantia de que estas propriedades serão preservadas através dos sucessivos refinamentos. Esta proposta foi mais tarde estendida por Suzuki e Murata (1982) para permitir refinamentos tanto nas transições quanto nos lugares.

O conceito de hierarquia em redes foi revisto em (GIRAULT; VALK, 2001), desta vez permitindo a representação de subredes de uma forma geral. A definição de subrede usada nessa abordagem permitia que elementos de diferentes tipos (passivos ou ativos) tivessem relação com a rede no nível abstrato superior. Este tipo de definição de hierarquia, apesar de ser muito geral, não garante que teremos uma rede válida em todos os níveis de abstração e portanto, verificações em diferentes níveis de detalhamento do projeto não seriam possíveis.

Entre as propostas recentes de extensões de redes de Petri que suportam características de hierarquia, a abordagem proposta em (VALETTE, 1979) é a mais utilizada como por exemplo nas redes PRES+ (CORTES; ELES; PENG, 2003) e TMPN (LAKOS; PETRUCCI, 2007).

Por definição (FOYO, 2001), a rede GHENeSys suporta a modelagem de sistemas em diferentes níveis de abstração. Como em (SUZUKI; MURATA, 1982), subredes podem substituir tanto lugares quanto transições. A definição de "blocos bem formados" em (VALETTE, 1979) foi relaxada ao introduzir a definição de subredes próprias, e foi provada a conservação de propriedades tais como limitação, vivacidade, além da conservação dos invariantes de lugar e transição.

Com a incorporação do tempo na rede GHENeSys, a definição dos elementos 
macros deverá sofrer ligeiras mudanças. A seguir apresentamos as novas definições para estes elementos.

Definição 43 (Elemento macro). Um elemento macro $\pi_{x} \in B \cup A$ que pertence a uma rede $\boldsymbol{G H E N e S y s} G=\left(L, A, F, K, \Pi, C_{0}, \tau\right)$ com $\Pi\left(\pi_{x}\right)=1$, representa uma subrede $G_{x}=\left(L_{x}, A_{x}, F_{x}, K_{x}, \Pi_{x}, C x_{0}, \tau_{x}\right)$ com $L_{x}=B_{x} \cup P_{x}$ onde:

1. $G_{x}$ tem dois elementos in e out, tal que ${ }^{\bullet}$ in $=\emptyset$ e out ${ }^{\bullet}=\emptyset$;

2. $\pi_{x} \in B \rightarrow$ in, out $\in B$ e $\pi_{x} \in A \rightarrow$ in, out $\in A$;

3. $\operatorname{dom}\left(F_{x}\right) \cap \operatorname{cod}\left(F_{x}\right)=L_{x} \cup A_{x}$;

4. se $\pi_{x} \in B, \forall M_{i} \in R\left(C x_{0}\right) \bullet$ out $\in \operatorname{enb}\left(M_{i}\right)$ sse $M x_{0}($ in $)>0$;

5. se $\pi_{x} \in A, \forall M_{i} \in R\left(C x_{0}\right)$ out $\in \operatorname{enb}\left(M_{i}\right)$ sse $\forall b \in \bullet^{\text {in }}, M(b)>0$;

6. o melhor tempo de execução $\sigma_{l}$ e o pior tempo de execução $\sigma_{u}{ }^{4}$ satisfazem $l \leq \sigma_{l}$ e $\sigma_{u} \leq u$ onde $\tau\left(\pi_{x}\right)=[l, u]$.

Os items 1, 2 e 3 referem-se à estrutura da subrede, que começa e termina com elementos do mesmo tipo que a subrede substitui. Também garante que não existem elementos desconectados na subrede. Os items 4 e 5 garantem que só as marcas presentes na entrada da subrede podem evoluir até alcançar a saída. Isto permite estabelecer de forma segura os tempos mínimos e máximos em que uma marca transita através da subrede. Finalmente o item 6 especifica que os tempos mínimos e máximos de evolução das marcas através da subrede devem ser compatíveis com o intervalo ligado ao elemento que representa a subrede no nível abstrato superior.

Respeitando a definição anterior, a conservação de propriedades como limitação, vivacidade, invariantes de lugar e transição durante os sucessivos refinamentos continua valendo, como foi provado em (FOYO, 2001). Note-se que a definição de elemento macro continua a mesma do ponto de vista da sua estrutura, e os items 4 e 5 determinam que a subrede é viva, segundo a definição de elementos próprios usada neste mesmo trabalho.

\footnotetext{
${ }^{4}$ Estes tempos são medidos como o tempo da ocorrência da atividade out a partir da ocorrência de in no caso das atividades macro, e o tempo transcorrido até atingir uma marcação onde $M$ (out) é incrementada a partir do momento em que uma marcação com $M($ in $)>0$ foi alcançada, no caso dos lugares macro.
} 
Na definição anterior, o comportamento temporal da subrede foi ligeiramente relaxado, permitindo refinamentos onde os intervalos de tempo ligados aos elementos macro não seguem exatamente o comportamento temporal da subrede que estes representam. Este tipo de refinamento é definido como refinamento fraco.

Seguindo esse raciocínio, o refinamento forte é aquele onde os intervalos ligados ao elemento macro identificam de forma exata os limites superior e inferior dos tempos de execução da subrede que eles representam.

Dependendo do tipo de refinamento usado, a preservação da validade das fórmulas lógicas nos diferentes níveis de abstração será de vital importância para a aplicação de uma abordagem modular do processo de verificação de modelos.

Seja uma rede $G=\left(L, A, F, K, \Pi, C_{0}, \tau\right)$ e uma fórmula $\phi$. Admitimos que $\phi$ é válida em $G$, isto é, $G, s_{0} \models \phi$. Admita-se ainda que a rede $G$ tem um elemento macro $\pi_{x}$ que representa a subrede $G_{x}=\left(L_{x}, A_{x}, F_{x}, K_{x}, \Pi_{x}, C x_{0}, \tau_{x}\right)$.

Se este elemento macro for um box $\left(\pi_{x} \in B\right)$, então as proposições que representam a marcação do elemento $\pi_{x}$ não podem ser usadas em $\phi$. Se o elemento refinado for uma atividade, então não existem restrições para a fórmula $\phi$.

Denotaremos como $\Omega_{G}$ ao conjunto de todas as seqüencias ou caminhos que satisfazem a fórmula $\phi$ na rede $G$.

Resta tentar provar que na rede $G r$, resultado do refinamento do elemento $\Pi_{x}$ na rede $G$, a fórmula $\phi$ continua válida, ou seja, $G r, s_{0} \models \phi$.

Como na fórmula $\phi$ não existem proposições ligadas aos elementos da subrede Gr e o próprio elemento macro não faz parte da fórmula $\phi$, a validade desta fórmula só vai depender do comportamento temporal da subrede $G r$.

Supondo que a validade da fórmula $\phi$ depende de uma restrição temporal entre dois estados da rede $G$, podemos considerar, sem perda de generalidade, que a seguinte restrição temporal: $\phi_{1}-\phi_{2} \leq c$.

Admita-se que $\Omega_{G}$ denota o conjunto dos caminhos possíveis entre estes estados. Então é possível escolher dentre estes caminhos possíveis o que passa pela atividade macro $\pi_{x}^{5}$ e tem a maior duração possível em todas as transições de estado. Denominaremos este caminho como $\omega_{i}$.

Considere-se então que $\omega_{i} \in \Omega_{G}$ é definido da seguinte forma:

\footnotetext{
${ }^{5} \mathrm{O}$ mesmo argumento pode ser usado se $\Pi_{x}$ for um box só que nesse caso usaríamos o conjunto das pós-condições de $\Pi_{x}$
} 


$$
\omega_{i}=s_{0} \stackrel{a_{1}, u_{1}}{\longrightarrow} s_{1} \stackrel{a_{2}, u_{2}}{\longrightarrow} \ldots \stackrel{a_{n-1}, u_{n-1}}{\longrightarrow} s_{n-1} \stackrel{a_{n}, u_{n}}{\longrightarrow} s_{n}
$$

com $s_{0} \in \phi_{1}$ e $s_{n} \in \phi_{2}$ e como $G, s_{0} \models \phi$ então $\sum_{i=0}^{n} u_{i} \leq c$.

Como a subrede $G_{x}$ é viva, de acordo com a definição 43 , então podemos garantir que existe pelo menos um caminho $\omega_{j}$ em $G r$. Este caminho estaria definido da seguinte forma:

$$
\omega_{j}=s_{0} \stackrel{a_{1}, u_{1}}{\longrightarrow} s_{1} \stackrel{a_{2}, u_{2}}{\longrightarrow} \ldots s_{x 0} \stackrel{a_{x 1}, d_{x 1}}{\longrightarrow} s_{x 1} \ldots \stackrel{a_{x k}, d_{x k}}{\longrightarrow} s_{x k} \ldots \stackrel{a_{x+1}, u_{x+1}}{\longrightarrow} \ldots s_{n-1} \stackrel{a_{n}, u_{n}}{\longrightarrow} s_{n}
$$

onde o elemento $\pi_{x}$ foi substituído pela seqüencia de $k$ atividades que levam do elemento de entrada ao elemento de saída da subrede $G_{x}$.

Como $\sum_{i=1}^{k} d_{x i} \leq u_{x}$, pela definição 43 , os novos estados $s_{x 0} \ldots s_{x k}$ não modificam a marcação fora da subrede $G_{x}$ e os elementos que pertencem a subrede $G_{x}$ não fazem parte da fórmula, portanto podemos garantir que a seqüencia $\omega_{j}$ satisfaz a fórmula $\phi$.

Note-se que permutações ou relações de ordem parcial das atividades que pertencem a subrede $G_{x}$ não afetam a lógica da fórmula porque estas não modificam estados da rede de alto nível.

Isto nos leva a formular a seguinte proposição:

Proposição 44. Dada uma rede $\boldsymbol{G H E N e S y s} G=\left(L, A, F, K, \Pi, C_{0}, \tau\right)$ e uma fórmula temporal $\phi$ tal que $G \models \phi$, se refinamos o elemento macro $\pi_{x}$ de modo que a definição 43 é satisfeita na rede $G_{r}$ resultado do refinamento do elemento $\pi_{x}$, a fórmula $\phi$ continua sendo válida.

O mesmo processo pode ser analisado de maneira inversa. Suponha que temos uma rede $G$ onde uma determinada fórmula $\phi$ é válida. Admitamos que existe uma subrede $G_{x}$ dentro da rede $G$ que cumpre a definição 43 e portanto esta subrede pode ser substituída pelo elemento macro $\pi_{x}$. Claramente, nenhum elemento de $G_{x}$ pode aparecer na fórmula $\phi$.

Considere-se, sem perda de generalidade, fórmulas com restrição de tempo mínimo ${ }^{6}$.

Seja o caminho mais demorado que contém atividades pertencentes a subrede $G_{x}$ que satisfaz $\phi$ em $G$, denotado por $\omega_{i}$. Obviamente as atividades que pertencem à rede $G_{x}$ em nada mudam a lógica da fórmula $\phi$. Substituindo a rede $G_{x}$

\footnotetext{
${ }^{6} \mathrm{~A}$ validade destas fórmulas depende dos tempos máximos das possíveis seqüencias
} 
pelo elemento macro correspondente na rede $G$ observando a definição 43 tem-se que a somatória do tempo das atividades em $\omega_{i}$ que pertencem a $G_{x}$ é menor ou igual ao tempo máximo da atividade que substitui à rede $G_{x}$, portanto $G \models \phi$ continua valendo.

Proposição 45. Dada uma rede $\boldsymbol{G H E N e S y s ~} G=\left(L, A, F, K, \Pi, C_{0}, \tau\right)$ e uma fórmula temporal $\phi$ tal que $G \models \phi$, se existe uma subrede $G_{x}$ dentro da rede $G$ que cumpre a definição 43 e nenhum dos seus elementos aparece na fórmula $\phi$, então esta pode ser substituída pelo elemento macro $\pi_{x}$ na rede de $G$ sem alterar a validade da fórmula $\phi$.

As proposições anteriores estabelecem a manutenção da validade das fórmulas através dos refinamentos ainda que nada tenha sido dito sobre a propagação de fórmulas do tipo $G \not \models \phi$. Para que as fórmulas preservem o seu resultado, seja verdadeiro ou falso, a semântica do refinamento forte deve ser garantida. Só a precisão dos intervalos dos elementos macro pode garantir a preservação das fórmulas nos diferentes níveis de abstração.

O argumento para este tipo de declaração vem da possibilidade de uma fórmula, considerada falsa no nível abstrato superior, ser verdadeira logo do refinamento por causa do relaxamento dos limites temporais do elemento macro.

Seguindo este argumento e levando em consideração as proposições anteriores é possível formular o seguinte teorema:

\section{Teorema 46 (Manutenção da validade das fórmulas durante o processo} de refinamento). Dada uma rede GHENeSys $G=\left(L, A, F, K, \Pi, C_{0}, \tau\right)$ e uma fórmula temporal $\phi$, a validade da fórmula $\phi$ é mantida em qualquer nível de abstração sempre que os elementos macros respeitarem a definição 43 e a semântica forte do refinamento.

A prova deste teorema segue os argumentos apresentados nas proposições 44 e 45 assim como dos argumentos que precedem o teorema 46 .

O teorema 46 é a base para a aplicação de uma abordagem composicional na verificação de sistemas na proposta apresentada neste trabalho. Suponha que temos um sistema modelado usando GHENeSys e um conjunto de fórmulas que precisam ser verificadas. Como foi estabelecido, a parte mais custosa do algoritmo de verificação é o cálculo do espaço de estados. Sendo assim, seria conveniente reduzir o cálculo do espaço de estados a um subconjunto próprio com respeito às 
fórmulas que precisam ser verificadas. Esta idéia é a mesma em que ampara as abordagens simbólicas e composicionais.

O modelo do sistema pode então ser reduzido da seguinte forma:

1. Selecione uma subrede que possa ser substituída por um elemento macro de modo que nenhum dos seus elementos apareça nas fórmulas a verificar.

2. Determine as classes de estados da subrede e construa o grafo das transições temporizadas correspondente. Note que ao construir este grafo é possível determinar se a rede é viva.

3. Se a subrede selecionada for viva, execute sobre o GTT obtido uma variante do algoritmo DiffCheck para determinar a diferença de tempo mínima e máxima entre a entrada e a saída da subrede.

4. Substitua esta subrede pelo elemento macro com intervalo estático de tempo igual ao determinado no item anterior.

Estes passos devem ser repetidos até que não existam subredes que possam ser substituídas por elementos macro. Neste ponto temos uma rede onde a verificação das fórmulas pode ser feita de forma eficiente.

Outra decorrência deste teorema é a possibilidade de verificação das fórmulas nos níveis mais abstratos, sem a necessidade de um refinamento excessivo dos componentes do sistema sempre que se tenha informação suficiente do comportamento temporal dos mesmos.

\subsection{Modificação no diagrama de classes da rede GHENeSys}

Com a inclusão do tempo na rede GHENeSys o diagrama de classes desta rede sofre algumas modificações. A figura 4.2 mostra o novo diagrama de classes padrão usado no desenvolvimento do ambiente GHENeSys.

Entre as modificações no diagrama de classes está a introdução da classe Net e a classe Arc. A classe Net é resultado da agregação das classes Arc, Place e Activity. Por sua vez, a classe Token foi relacionada à classe Place.

A classe Arc tem como única funcionalidade o relacionamento entre os elementos do conjunto dos lugares e as atividades, que são representados respectivamente pelas classes Place e Activity, além de guardar o peso de cada arco. 


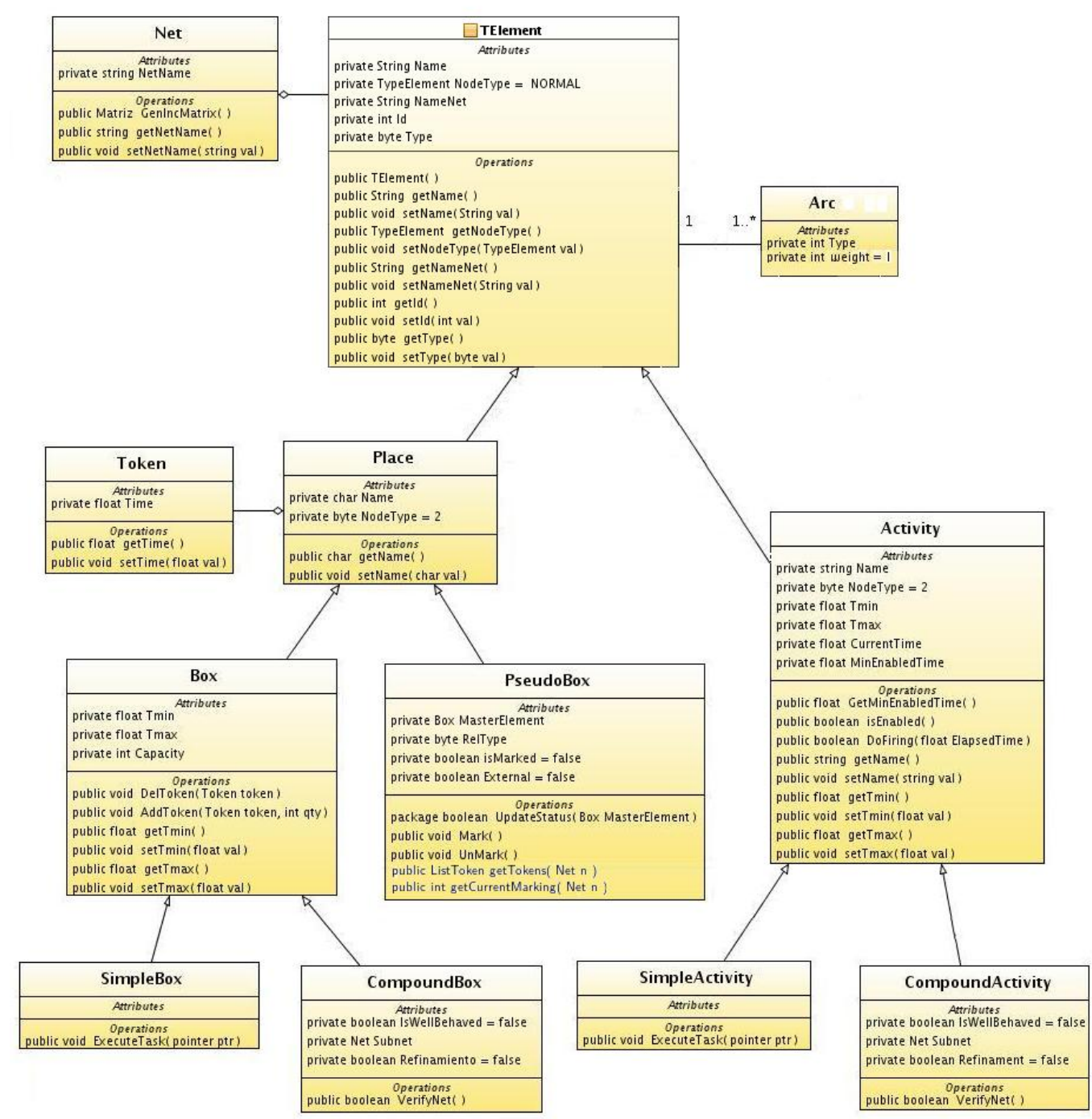

Figura 4.2: Novo Diagrama de Classes da rede GHENeSys

Uma mudança fundamental foi a inclusão da operação GenIncMatriz na classe Net. Este método gera a matriz de incidência da rede até o nível de refinamento desejado, o que é declarado através do atributo Refinement presente nos elementos macros CompoundBox e CompoundActivity. Nestas classes foi incluído também o método VerifyNet que atualiza o atributo isWellBehaved indicando se a subrede ligada ao elemento macro em questão satisfaz completamente a definição 43.

Como os pseudo-boxes podem representar elementos que são boxes em outras redes foi acrescentado na classe pseudo-box o método GetCurrentMarking o qual permite obter a marcação do elemento referenciado pelo atributo MasterElement. Este método é usado nas rotinas que determinam se uma determinada atividade está habilitada ou não. 


\section{Aplicações e Estudo de Caso}

Aplicações práticas podem ser encontradas nos sistemas embarcados, ou mais genericamente, nos sistemas de tempo real, onde este tipo de abordagem é mais freqüentemente utilizada. Existem ainda outros campos de aplicação onde um ambiente como o GHENeSys com os algoritmos de verificação com tempo denso pode ser bastante útil, mas que não foram ainda explorados devidamente. Portanto, o objetivo é apresentar uma proposta que não seja aderente a nenhuma classe de problemas em especial e que se ajuste a qualquer situação de modelagem, design e verificação.

Neste capítulo abordaremos algumas dessas aplicações assim como a forma de utilizar a técnica da verificação de modelos proposta neste trabalho.

\subsection{Escalonamento de workflows com restrições de tempo}

Sistemas de gerenciamento de workflows (WFMS pelas suas siglas em inglês) contribuem ao melhoramento dos processos de negócios através da automação das tarefas, coletando informações precisas para executar uma função de trabalho, e integrando informação as empresas (WFMC, 1996).

Neste conjunto de aplicações existe um grupo que demanda o gerenciamento do tempo e por isto tem recebido uma atenção especial por parte dos pesquisadores da área. Entre as abordagens usadas na modelagem de workflows com restrições de tempo podemos citar as seguintes:

Eder, Panagos e Rabinovich (1999) propuseram um grafo de workflow temporizado para modelar WFMSs. Neste trabalho foi introduzida uma técnica para escalonar as atividades de forma que todos os prazos fossem respeitados. Esta técnica foi logo melhorada em (EDER; GRUBBER; PANAGOS, 2000) com a introdução de uma rede denominada T-WfMC com a qual se pode modelar e especificar os WFMS. 
Nos workflows as atividades possuem durações determinísticas ou conhecidas o que favorece o uso de redes de Petri tipo Ranchandami na modelagem e análise de tais sistemas. Porém, o uso de redes tipo Merlin permite analisar o impacto de eventuais atrasos ou problemas de sincronização no cumprimento dos prazos estipulados para estes processos.

Nesta seção, vamos mostrar através de um exemplo como é possível usando a abordagem da verificação de modelos, obter resultados que podem ser usados na implementação dos algoritmos de escalonamento dos workflows.

A figura 5.1 mostra a especificação (ordem de precedência, duração de cada atividade e prazo limite estipulado para que dita atividade seja finalizada) de um fluxo de atendimento hospitalar.

\begin{tabular}{|c|c|c|}
\hline $\mathbf{a}_{\mathrm{i}}$ & dur $_{\mathrm{i}}$ & $\mathrm{d}_{\mathrm{i}}$ \\
\hline $\mathbf{a}_{0}$ & 5 & 5 \\
\hline $\mathbf{a}_{1}$ & 6 & $\mathbf{1 4}$ \\
\hline $\mathbf{a}_{2}$ & 4 & 21 \\
\hline $\mathbf{a}_{3}$ & 2 & 9 \\
\hline $\mathbf{a}_{4}$ & 2 & 8 \\
\hline $\mathbf{a}_{5}$ & 3 & 13 \\
\hline $\mathbf{a}_{6}$ & 3 & 18 \\
\hline $\mathbf{a}_{7}$ & 2 & 25 \\
\hline
\end{tabular}

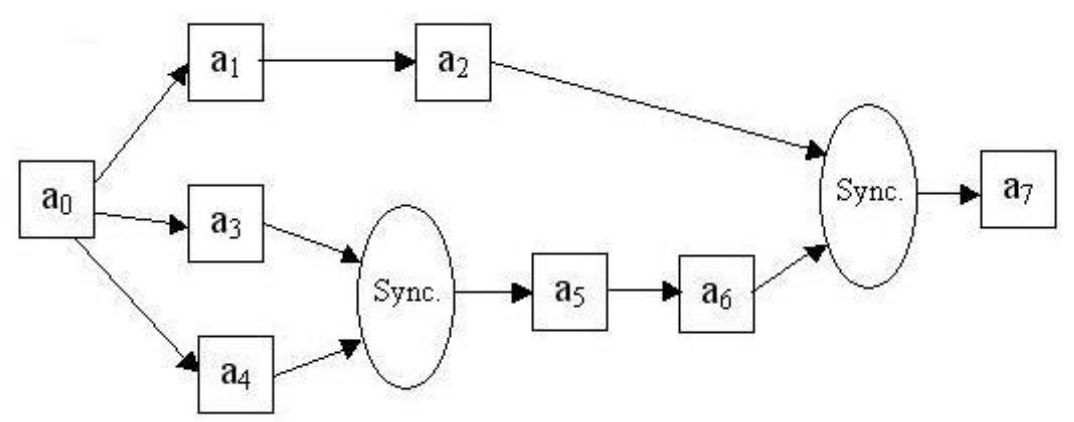

Figura 5.1: Exemplo de workflow

O exemplo da figura 5.1 é modelado usando uma RPT onde os intervalos de cada atividade refletem a duração, representado pelo EFT, e o prazo final para o término da sua execução contado a partir do término das atividades anteriores representado pelo LFT. A RPT é mostrada na figura 5.2.

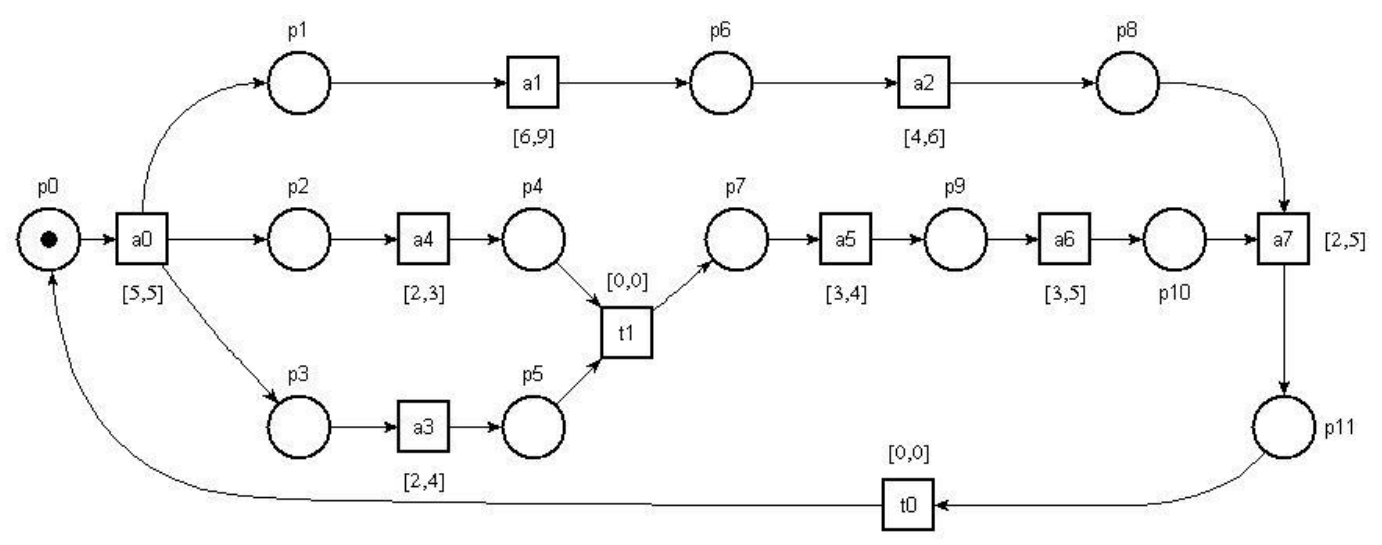

Figura 5.2: Modelo em RPT do exemplo de workflow da figura 5.1

Através da fórmula $\exists \diamond_{\leq 25} p 11$ é possível determinar se o processo é escalonável 


\begin{tabular}{|l|c|c|c|}
\hline Id & Atividade & Fórmula a verificar & Estado \\
\hline 1 & $a_{0}$ & $\forall \diamond_{\leq 5}(p 1 \wedge p 2 \wedge p 3)$ & $s_{0}$ \\
2 & $a_{1}$ & $\exists \diamond_{\geq 0} p 1 \rightarrow p 6$ & - \\
3 & $a_{1}$ & $\forall \diamond_{\leq 14} p 6$ & $s_{0}$ \\
4 & $a_{2}$ & $\exists \diamond_{\geq 0} p 6 \rightarrow p 8$ & - \\
5 & $a_{2}$ & $\forall \diamond_{\leq 21} p 8$ & $s_{0}$ \\
6 & $a_{3}$ & $\exists \diamond_{\geq 0} p 3 \rightarrow p 5$ & - \\
7 & $a_{3}$ & $\forall \diamond_{\leq 9} p 5$ & $s_{0}$ \\
8 & $a_{4}$ & $\exists \diamond_{\geq 0} p 2 \rightarrow p 4$ & - \\
9 & $a_{4}$ & $\forall \diamond_{\leq 8} p 4$ & $s_{0}$ \\
10 & $a_{5}$ & $\exists \diamond_{\geq 0} p 7 \rightarrow p 9$ & - \\
11 & $a_{5}$ & $\forall \diamond_{\leq 13} p 9$ & $s_{0}$ \\
12 & $a_{6}$ & $\exists \diamond_{\geq 0} p 9 \rightarrow p 10$ & - \\
13 & $a_{6}$ & $\forall \diamond_{\leq 18} p 10$ & $s_{0}$ \\
14 & $a_{7}$ & $\exists \diamond_{\geq 0}(p 8 \wedge p 10) \rightarrow p 11$ & - \\
15 & $a_{7}$ & $\forall \diamond_{\leq 25} p 11$ & $s_{0}$ \\
\hline
\end{tabular}

Tabela 5.1: Especificação em TCTL do exemplo de workflow

ou não. Nesse caso é fácil de ver que o sistema é escalonável mas se fosse usada uma fórmula mais restritiva, por exemplo, $\forall \diamond_{\leq 25} p 11$, uma verificação positiva desta fórmula garantiria que - sempre respeitando os prazos máximos para o término de cada tarefa prevista - as folgas previstas para as próximas tarefas são suficientes para garantir o cumprimento do prazo final.

Usando o verificador de modelos global, não basta apenas determinar se a fórmula é verdadeira. Isto poderia indicar apenas que a mesma é verdadeira em ao menos um estado o que não é suficiente para garantir a propriedade desejada. Neste caso deve-se consultar se a fórmula é verdadeira no estado inicial.

Uma técnica como esta pode ser usada para projetar escalonadores estáticos, que são aqueles onde as tarefas, as pre-condições e as durações máximas e mínimas são conhecidos desde a fase de projeto.

A especificação do sistema é representada na figura 5.1. O grafo representa a ordem de precedência para cada atividade e a tabela mostra os prazos definidos para cada atividade. Esta especificação pode ser interpretada pelas seguintes fórmulas temporais: A figura 5.3 mostra o resultado da verificação das propriedades da tabela 5.1 no verificador.

As fórmulas 2, 4, 6, 8, 10 são verdadeiras e as fórmulas 1, 3, 5, 7, 9, 11 são verdadeiras no estado inicial ( $s_{0}$ no caso) além de em outros estados. No caso específico da figura 5.3 são mostrados os estados onde a fórmula $\forall \diamond_{\leq 25} p 11$ é verdadeira. 


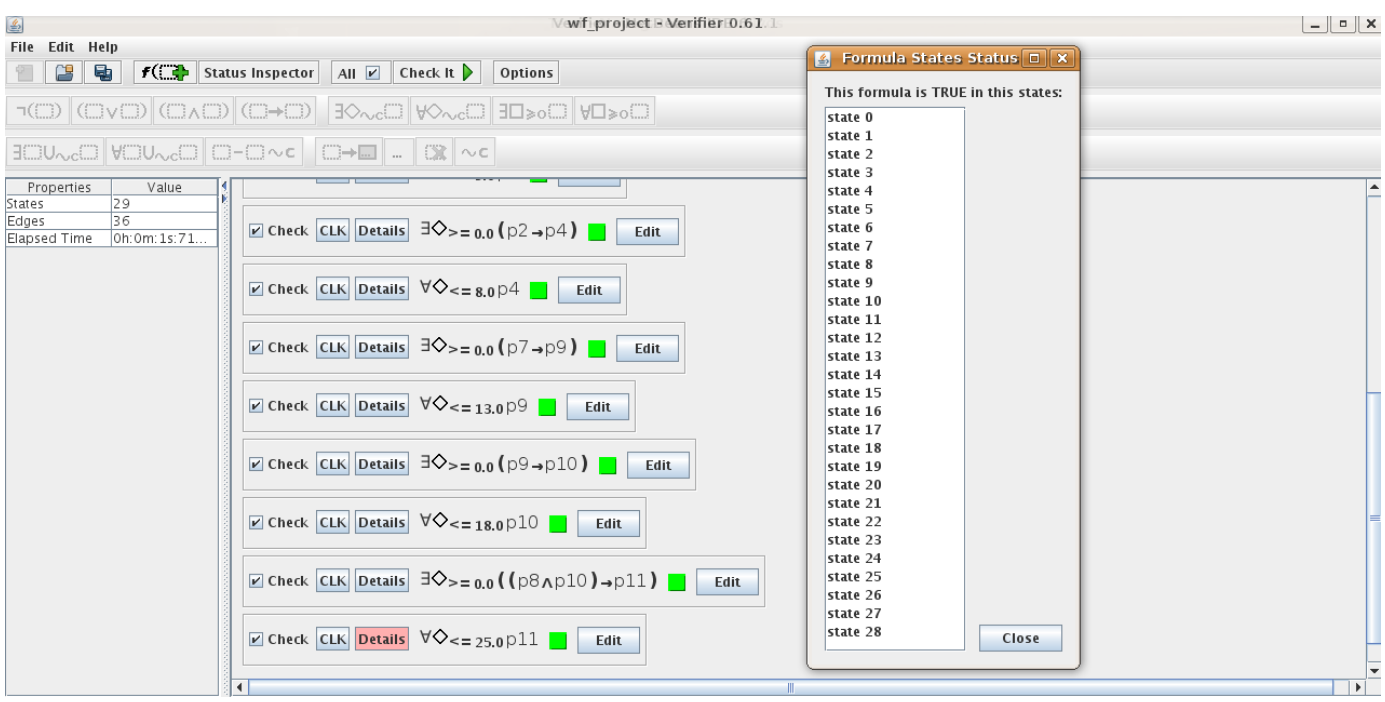

Figura 5.3: Resultados da verificação do exemplo da figura 5.1, ressaltando em que estados a fórmula $\forall \diamond_{\leq 25} p 11$ é verdadeira

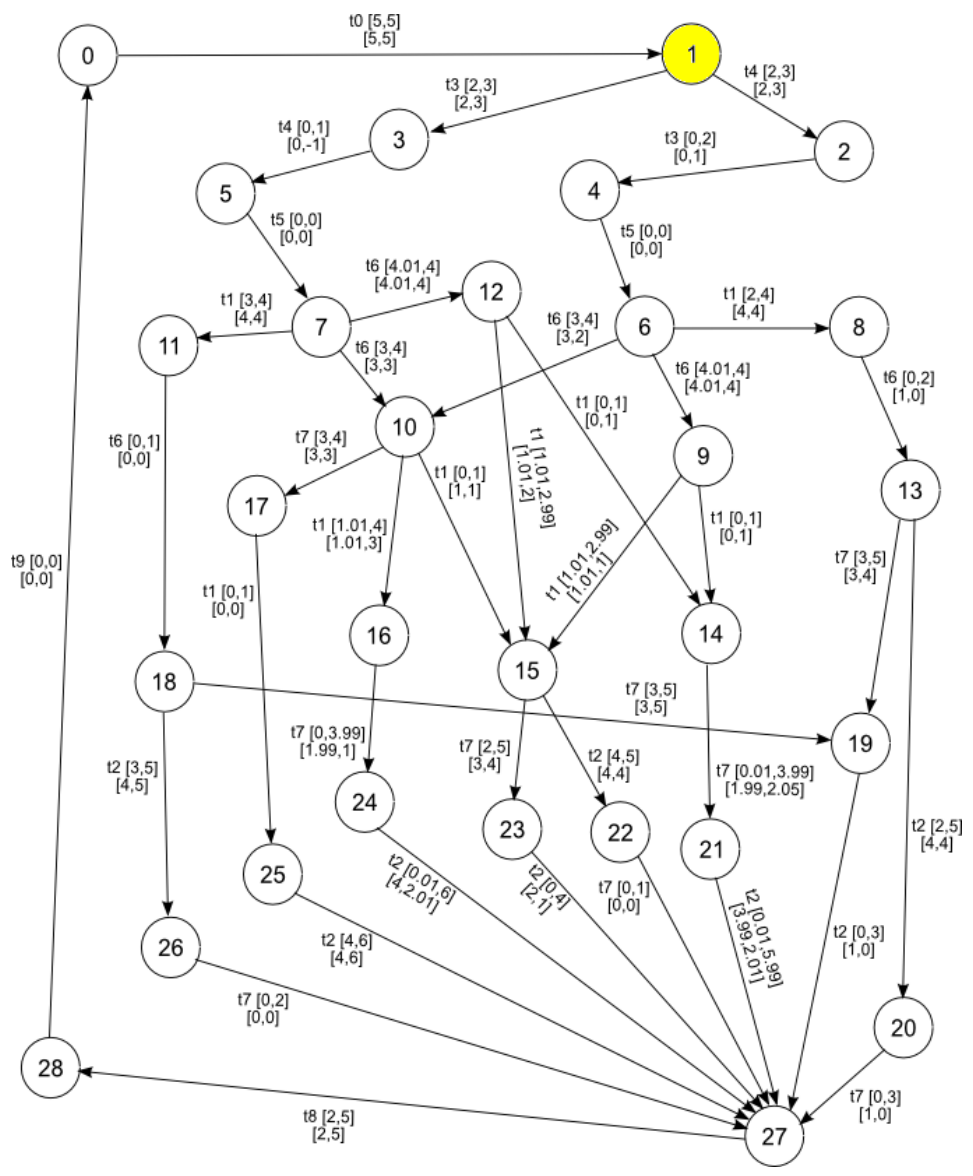

Figura 5.4: GTT do exemplo da figura 5.1

A figura 5.4 apresenta o GTT do exemplo da figura 5.1. Nesta figura, os estados onde a subfórmula $p 1 \wedge p 2 \wedge p 3$ é verdadeira estão sinalizados pela cor amarela. A figura 5.5 mostra o resultado da verificação da fórmula $\forall \diamond_{\leq 5}(p 1 \wedge$ $p 2 \wedge p 3)$. 


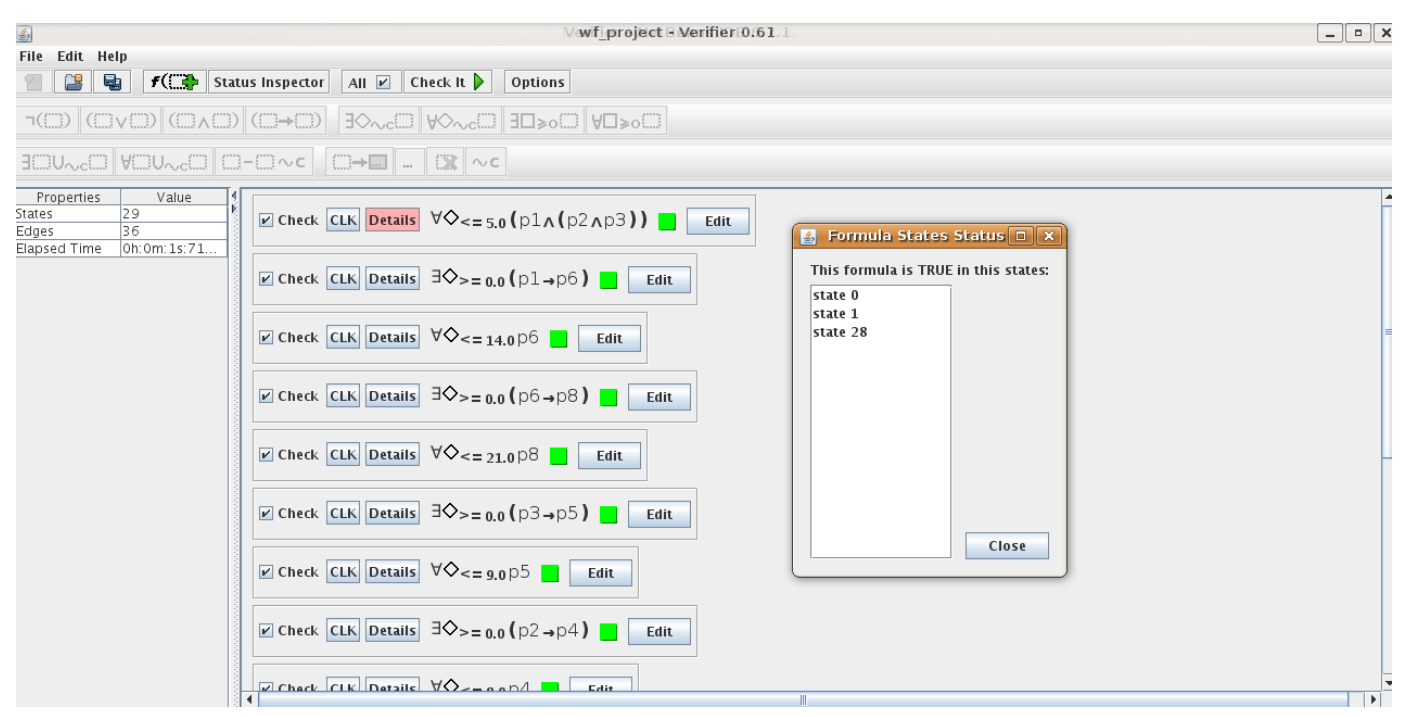

Figura 5.5: Estados onde a fórmula $\forall \diamond_{\leq 5}(p 1 \wedge p 2 \wedge p 3)$ é verdadeira

Nota-se que o estado 1 só pode ser atingido em um tempo menor ou igual a 5 unidades de tempo a partir dos estados 0 e 28.

\subsection{Verificação de sistemas com tempo}

Nesta seção mostra-se o uso das ferramentas desenvolvidas neste trabalho na verificação de um sistema de evaporação inicialmente apresentado em (HUUCK; LUKOSCHUS; LAKHNECH., 2001), (KOWALEWSKI; STURSBERG; BAUER, 2001) e modificado em (MACHADO et al., 2007).

Em (MACHADO et al., 2007) foram apresentados os resultados da verificação do sistema de evaporação usando a ferramenta UPPAAL, portanto utilizaremos como estudo de caso o sistema tal como descrito nesse trabalho, com o intuito de fazer uma comparação entre os resultados produzidos por este sistema e os da GHENeSys.

\subsubsection{Descrição do comportamento do estudo de caso}

O sistema de evaporação usado no estudo de caso é apresentado na Figura 5.6 e é composto pelos tanques 1 e 2, (sendo que no tanque 1 o líquido pode ser aquecido e misturado), um condensador, sensores de nível e válvulas on/off. No modo de operação normal o sistema deve se comportar da seguinte forma:

- O tanque 1 começa a encher abrindo as válvulas V1 e V2.

- O misturador começa a funcionar para promover a mistura das duas soluções. 


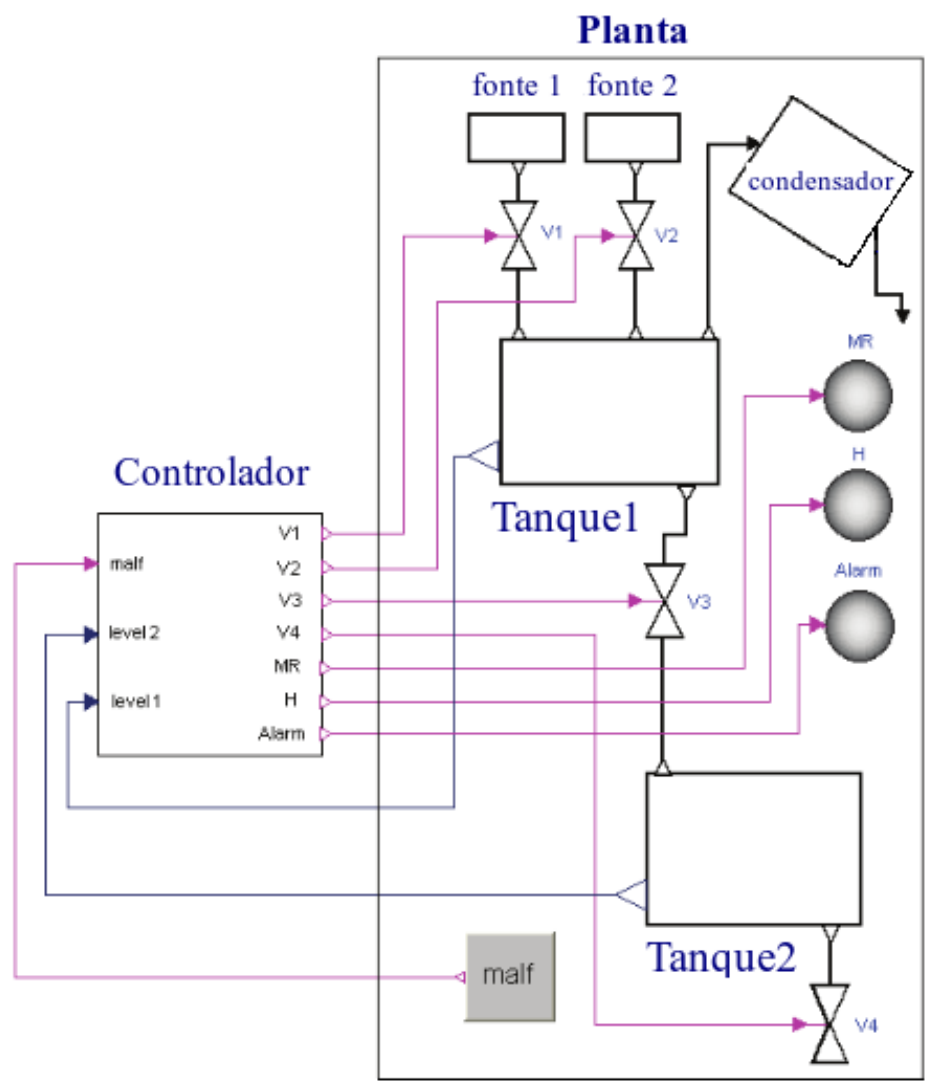

Figura 5.6: Sistema de evaporação. Malha fechada do sistema composto pelo controlador e a planta.

- Depois de 2 unidades de tempo, o aquecedor é ligado por um intervalo de 20 unidades de tempo para aumentar a temperatura da solução. Durante este período, parte do líquido é evaporado e resfriado no condensador. Neste ponto a solução deve ter alcançado a concentração requerida e o aquecedor deve ser desligado.

- As válvulas V1 e V2 são fechadas uma vez que o tanque 1 está cheio.

- O líquido no tanque 1 é transferido para o tanque 2 através da abertura da válvula V3.

- O misturador é desligado uma vez que o tanque 1 é esvaziado e a válvula V3 é fechada.

- A solução deve permanecer no tanque 2 por 32 unidades de tempo quando deve ser descartada abrindo a válvula V4.

Durante a operação normal do sistema falhas podem acontecer. No processo de evaporação o condensador pode falhar o que não permitiria que o vapor fosse resfriado causando aumento da pressão no condensador. Para evitar a explosão 
do condensador, o aquecedor deve ser desligado, restringindo a produção de vapor. Por causa disso a temperatura do líquido no tanque 1 pode diminuir o que levaria a solidificação da solução impedindo a transferência para o tanque 2 .

Portanto, a solução no tanque 1 deve ser transferida antes de que solidifique. Isto é feito através da abertura das válvulas V3 e V4.

Em caso de avaria do condensador é preciso ainda garantir alguns tempos de resposta do controlador levando em consideração as características do processo:

- No momento em que acontece a falha do condensador, este pode explodir se o vapor continua a ser produzido por 22 unidades de tempo a partir do começo da ocorrência da falha.

- Uma vez que o aquecedor é desligado a produção de vapor cessa após 12 unidades de tempo.

- Em caso de falta de vapor no tanque 1 por um intervalo de 19 unidades de tempo a solução é solidificada.

- Esvaziar o tanque 2 demora entre 0 e 26 unidades de tempo

- Encher o tanque 1 leva entre 1 e 6 unidades de tempo enquanto o esvaziamento é considerado instantâneo.

\subsubsection{Modelando o sistema usando GHENeSys}

Seguindo a recomendação em (DICESARE et al., 1994) o modelo do sistema de evaporação, a planta, e seu controle são modelados de forma separada. Para isto, o uso da rede GHENeSys na modelagem destes sistemas apresenta a vantagem do uso do pseudo-box como elemento de ligação.

A figura 5.7 mostra os modelos para os Tanques 1 e 2, a produção de vapor no condensador e sua relação com um possível mal funcionamento.

Os pseudo-boxes ps1, ps2 e ps3 representam o estado das válvulas e os pseudoboxes ps 8 e ps 9 representam o estado do aquecedor no sistema de evaporação. Estes elementos constituem saídas de controlador e estão ligados através da propriedade MasterElement da classe pseudo-box aos lugares da rede que modelam o comportamento do controlador:

Note que no modelo apresentado na figura 5.7 está sendo usado um "observador" para a situação em que a explosão do condensador pode acontecer. Esta situação é então verificada através da propriedade $\forall \square \neg C$ Cond. explodes. 


\begin{tabular}{|ccl|}
\hline pseudo-box & MasterElement & Comentário \\
\hline ps1 & V1V2 opened & válvulas 1 e 2 abertas \\
ps2 & V3 opened & válvula 3 aberta \\
ps3 & V4 opened & válvula 4 aberta \\
ps8 & Heater off & aquecedor desligado \\
ps9 & Heater on & aquecedor ligado \\
\hline
\end{tabular}

Tabela 5.2: Sinais do controlador presentes no modelo do evaporador

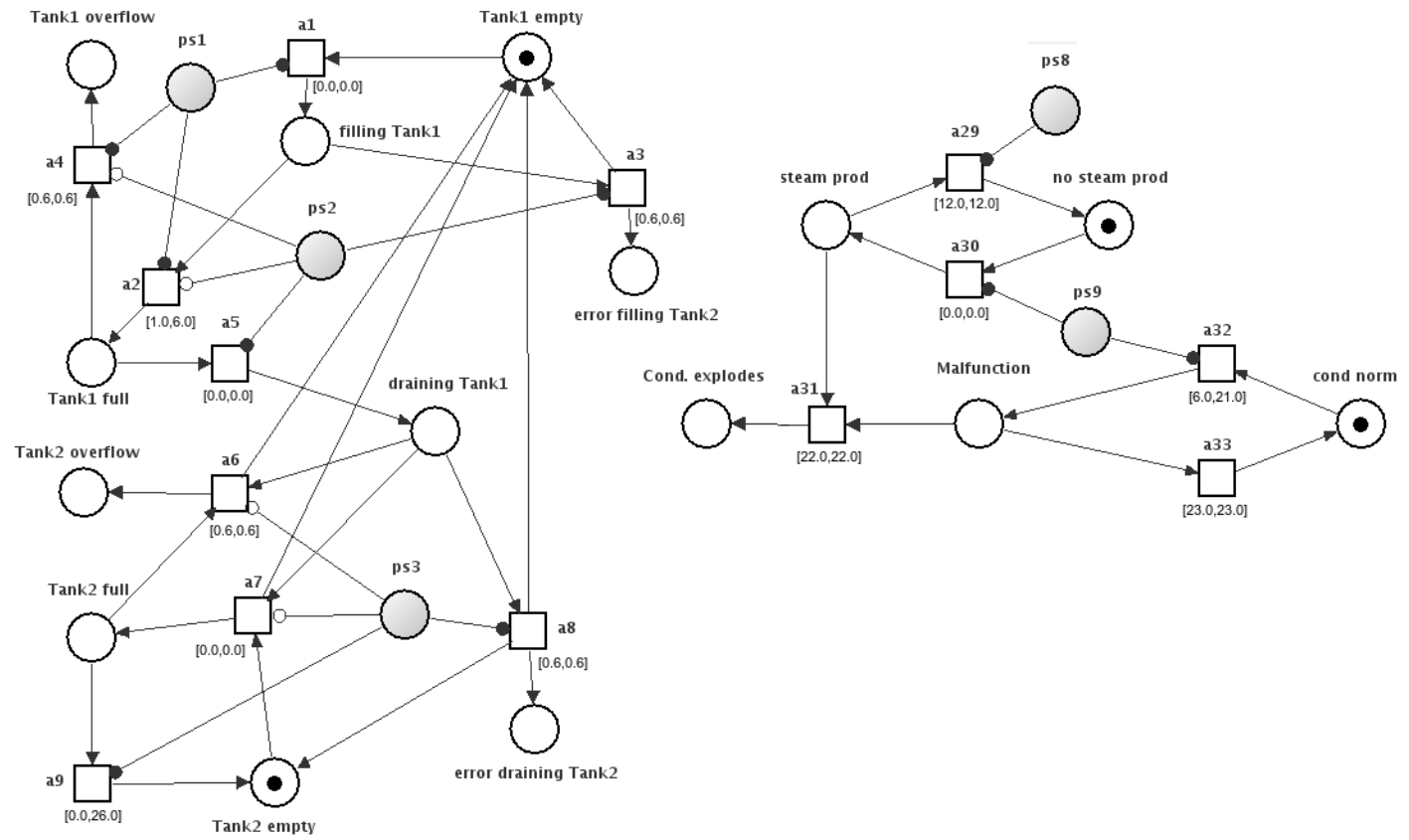

Figura 5.7: Modelo do Evaporador

A propriedade $\neg \exists($ Malfunction $\wedge$ steam prod $) U_{>22}$ no steam prod é uma outra forma de colocar esta situação sem a necessidade de inserir o lugar Cond. explodes, a transição a31 e os arcos ligados a ela.

O modelo do controle é apresentado na figura 5.8.

Como pode ser visto, os pseudo-boxes representam os sinais dos sensores do processo, como é mostrado na seguinte tabela:

\begin{tabular}{|ccl|}
\hline pseudo-box & MasterElement & Comentário \\
\hline ps4 & Malfunction & falha de funcionamento no condensador \\
ps5 & Tank2 empty & tanque 2 vazio \\
ps6 & Tank1 empty & tanque 1 vazio \\
ps7 & Tank1 full & tanque 1 cheio \\
\hline
\end{tabular}

Tabela 5.3: Sinais dos sensores do processo presentes no modelo do controlador 


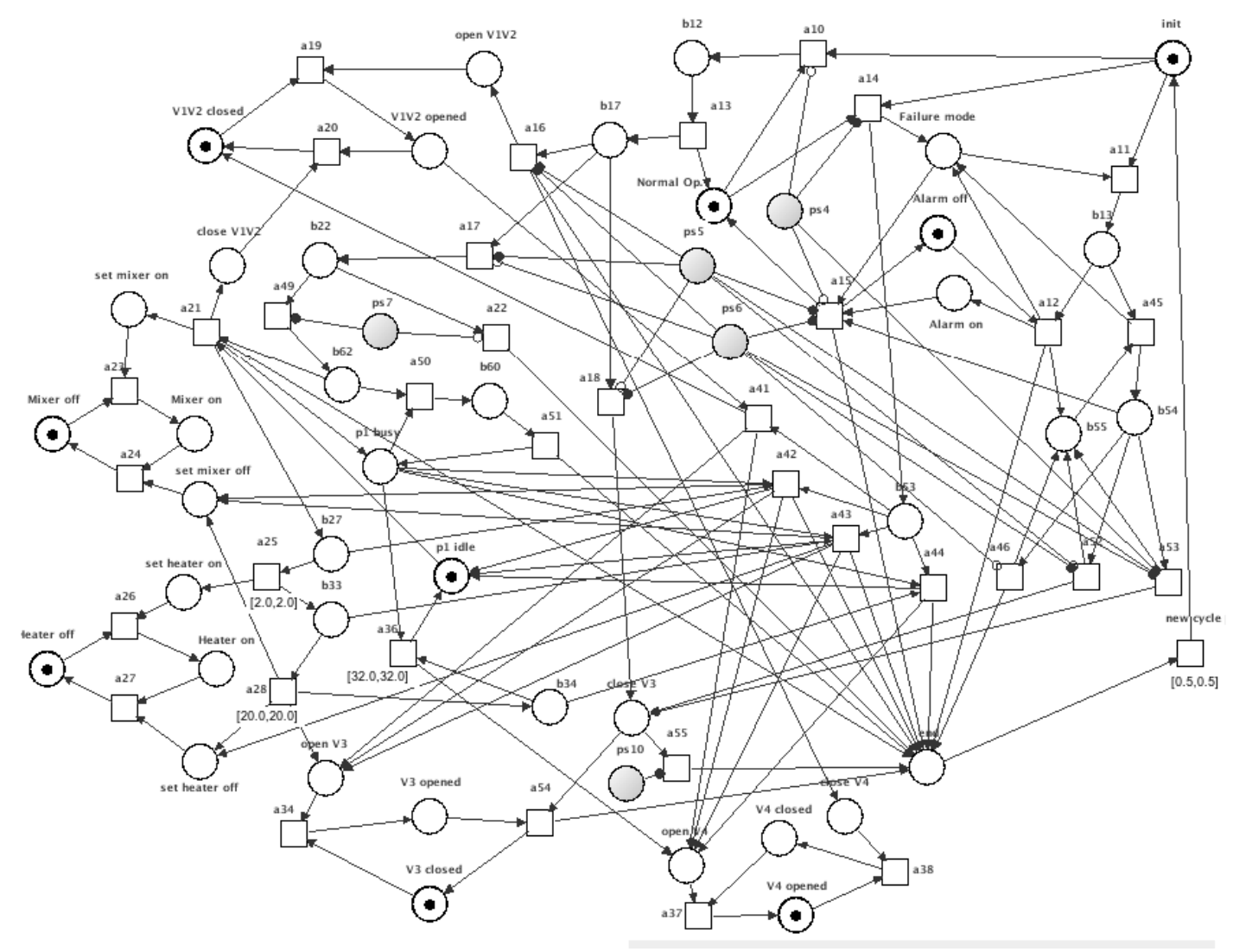

Figura 5.8: Modelo do controlador

\subsubsection{Formalizando a especificação usando a TCTL}

Uma vez que o modelo do sistema é concluído é possível formalizar a especificação do sistema. Note que é preciso conhecer as proposições atômicas, que são os lugares da rede, para poder definir as fórmulas.

A especificação do sistema de evaporação como a de qualquer outro sistema pode ser dividida em dois grupos de propriedades: as de vivacidade ou inevitáveis e as de segurança. As primeiras se referem a situações ou estados que devem ser atingidos para que o sistema possua as funcionalidades previstas. As segundas referem-se a situações que não podem ser atingidas porque levariam o sistema a uma condição crítica ou não desejada (restrições).

A tabela 5.4 mostra as fórmulas TCTL que expressam formalmente a especificação do sistema em apreço.

A propriedade 1 se refere à não existência de deadlocks no funcionamento do controlador. As propriedades 2 à 8 referem-se a propriedades de segurança ou situações que o controlador deve evitar que aconteçam. As propriedades 9 à 16 referem-se a propriedades de vivacidade ou funcionalidade que identificam o comportamento desejado para o sistema. 


\begin{tabular}{|c|c|}
\hline Id & Fórmula TCTL \\
\hline 1 & $\forall \square($ init $\rightarrow \forall \diamond$ end $)$ \\
\hline 2 & $\forall \square \neg$ Cond. explodes \\
\hline 3 & $\forall \square \neg$ Tank 1 over flow \\
\hline 4 & $\forall \square \neg$ Tank 2 overflow \\
\hline 5 & $\neg \exists$ no steam prod $\wedge($ filling Tank $1 \vee$ Tank 1 full $) U_{>19}$ steam prod \\
\hline 6 & $\forall \square \neg$ error filling Tank2 \\
\hline 7 & $\forall \square \neg($ error draining Tank $2 \wedge \neg$ Malfunction $)$ \\
\hline 8 & $\forall \square \neg($ Tank1 empty $\wedge$ Heater on $)$ \\
\hline 9 & $\forall \diamond_{\geq 0}\left[(\right.$ Tank 1 empty $\wedge$ Tank 2 empty $) \rightarrow \forall \diamond_{\leq 0.5} V 1$, V2 opened $]$ \\
\hline 10 & $\forall \diamond_{\geq 0}\left(\right.$ Tank 1 full $\rightarrow \forall \diamond_{\leq 0.5}$ Mixer on $)$ \\
\hline 11 & $\forall \diamond_{\geq 0}^{-}$Tank 1 full $\rightarrow \forall \diamond_{\leq 0.5} V 1, V 2$ closed \\
\hline 12 & $\forall \diamond_{\geq 0}\left[(\right.$ Tank 1 full $\wedge$ Heater of $f) \rightarrow \forall \diamond_{\leq 0.5} V 3$ opened $]$ \\
\hline 13 & $\forall \diamond_{\geq 0}\left[\right.$ Tank 1 empty $\rightarrow \forall \diamond_{\leq 0.5}(V 3$ closed $\wedge$ Mixer of $\left.f)\right]$ \\
\hline 14 & $\exists$ Tank 2 full $U_{\geq 32} V 4$ opened \\
\hline 15 & $\exists \diamond_{\geq 0}\left[\right.$ Tank 1 full $\wedge(\neg$ Mixer on $) \wedge\left(\forall\right.$ Heater on $U_{\geq 20}$ Heater of $\left.\left.f\right)\right]$ \\
\hline 16 & {$[\neg$ Malfuncion $\vee($ Tank 1 empty $\wedge$ Tank 2 empty $)] \rightarrow$ Alarm of $f$} \\
\hline
\end{tabular}

Tabela 5.4: Especificação em TCTL do funcionamento do sistema de evaporação

\subsubsection{Resultados do processo de verificação formal}

O GTT correspondente ao exemplo do sistema de evaporação foi construído em 28m:28s:452ms num computador Pentium Core 2 Duo a 2.66GHz e 2Gb de RAM.

O resultado deste processo pode ser observado na figura 5.9

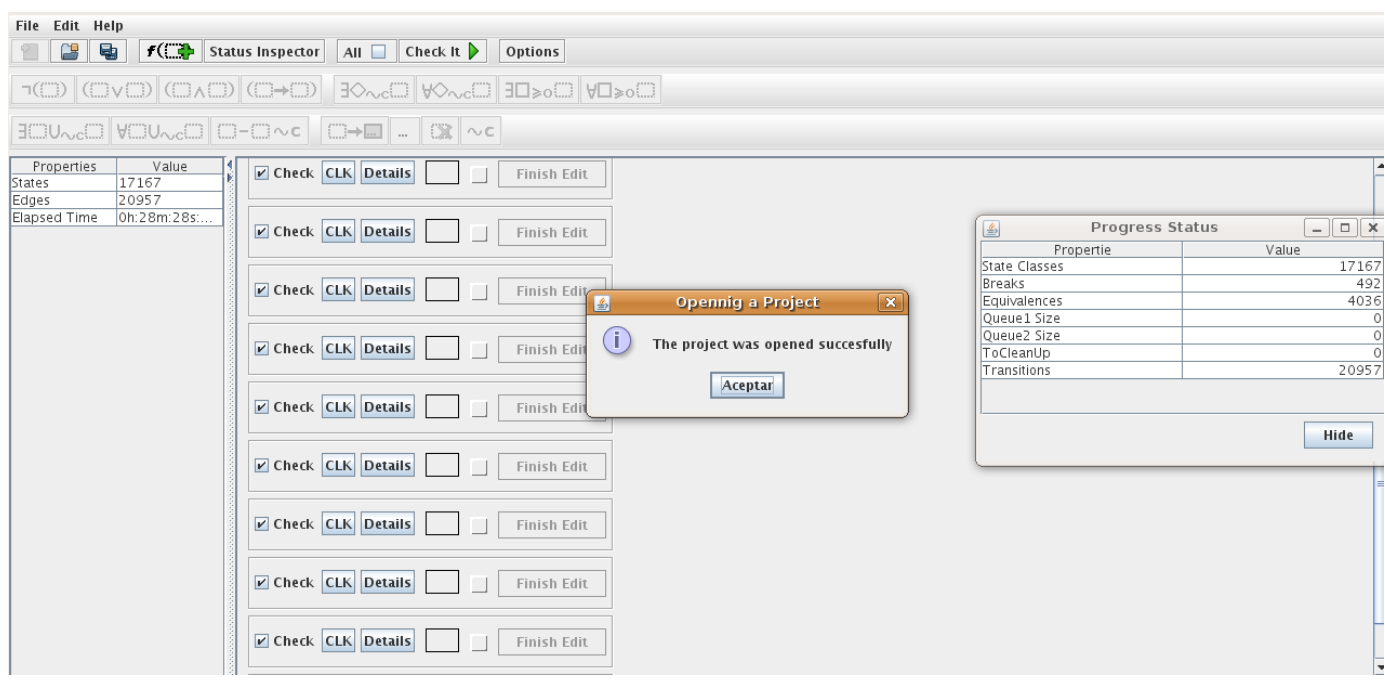

Figura 5.9: Resultado do computo das classes de estados com restrições para o sistema de evaporação

Uma vez computado o GTT, foram editadas as fórmulas TCTL que representam a especificação do sistema de evaporação (Ver tabela 5.4). As figuras 5.10 e 5.11 mostram o resultado do processo de verificação das fórmulas temporais que 
formam a especificação.

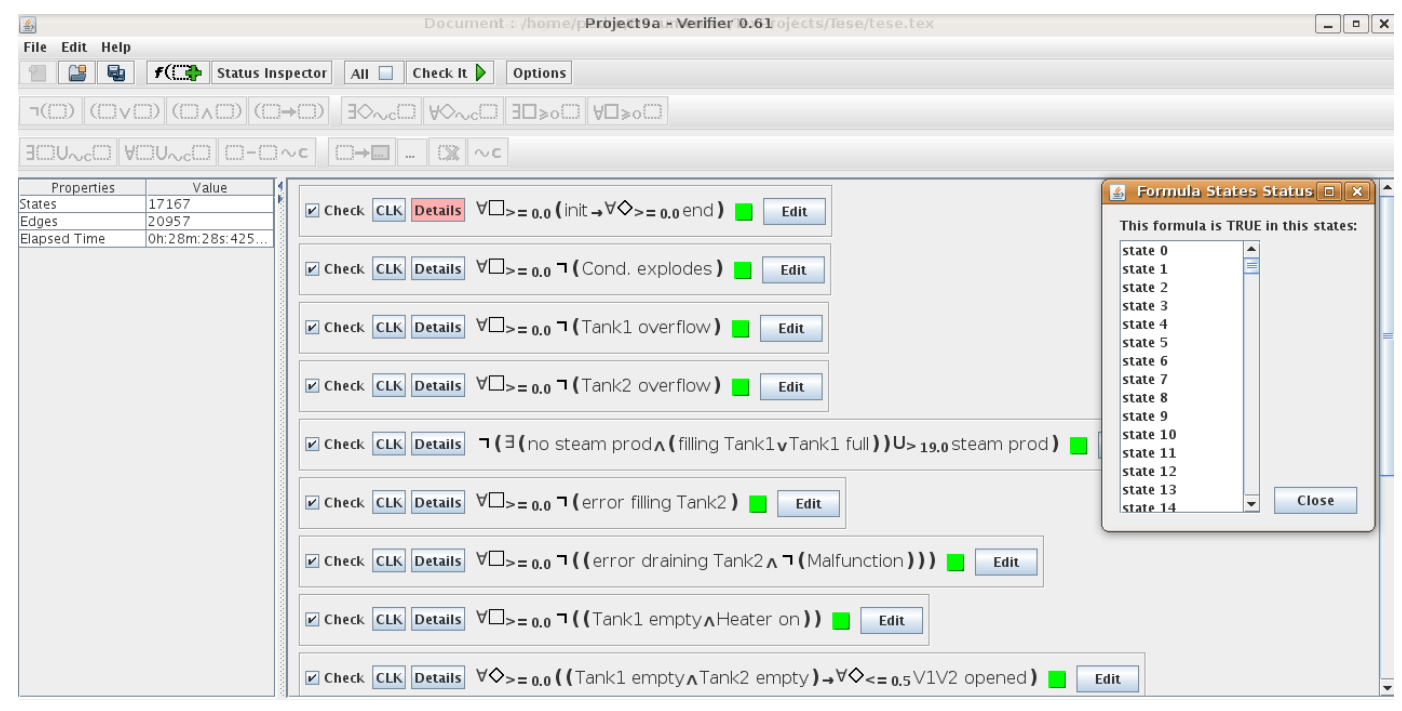

Figura 5.10: Resultado da verificação das propriedades de segurança para o sistema de evaporação

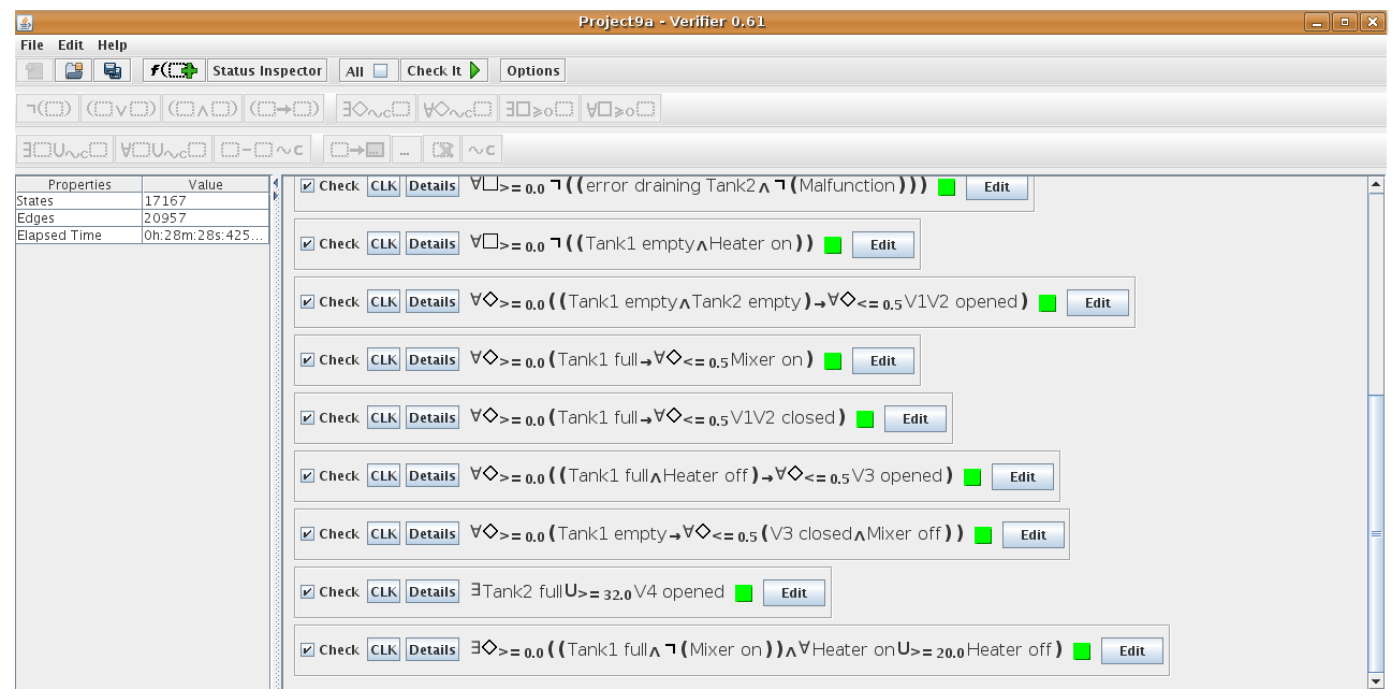

Figura 5.11: Resultado da verificação das propriedades de vivacidade para o sistema de evaporação

A determinação da validade ou não das 16 propriedades foi determinada em $3 m: 16 s$ sendo que todas resultaram válidas como pode-se apreciar nas figuras 5.10 e 5.11 .

A partir da rede que representa o comportamento do controlador pode ser desenvolvido o programa do CLP. O processo de tradução ainda que não é automático pode ser realizado com segurança, note que apenas recursos disponíveis nos CLP's tais como temporizadores e interrupções além das instruções de controle básicas são necessários na implementação do programa a partir do modelo. 


\subsection{Comparação dos algoritmos de geração das classes de estados}

A seguir apresenta-se uma comparação entre os algoritmos mais utilizados na geração das classes de estados que preservam o comportamento ramificado. Os algoritmos usados nesta comparação são: o algoritmo que gera as classes atômicas incorporado na ferramenta TINA, o proposto pelo Yoneda e Ryuba (1998) e o proposto nesta tese. Os casos usados na comparação foram os exemplos propostos em (YONEDA; RYUBA, 1998).

Em (YONEDA; RYUBA, 1998) apresentaram quatro exemplos de redes de Petri Temporizadas usaremos como base de comparação entre o tamanho do grafo de estados gerado usando o algoritmo proposto nesse trabalho e no algoritmo do grafo das regiões proposto em (ALUR; COURCOUBETIS; DILL, 1993). As redes escolhidas para realizar estes experimentos são mostrados na figura 5.12 .

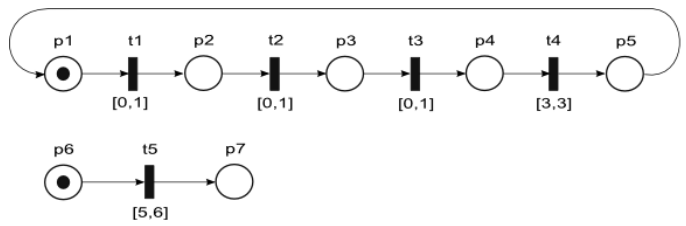

(a) Rede 1

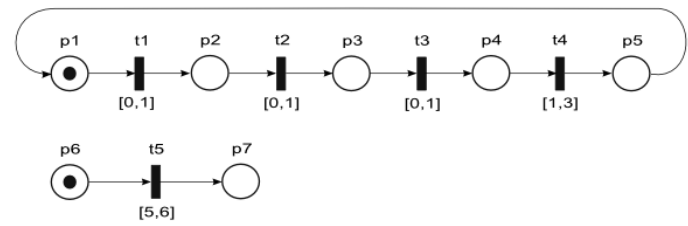

(b) Rede 2

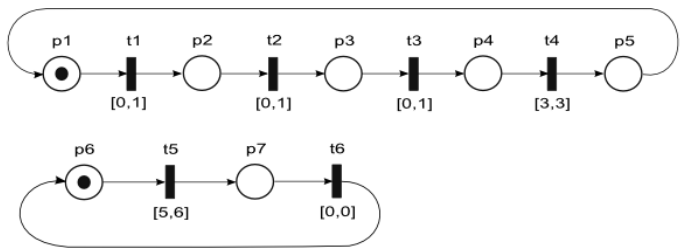

(c) Rede 3

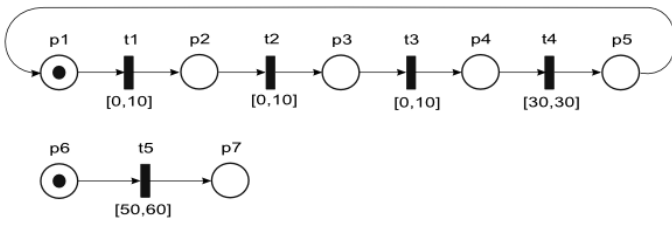

(d) Rede 4

Figura 5.12: Exemplos de rede de Petri temporizadas usadas nos experimentos de Yoneda e Ryuba (1998)

Os experimentos foram realizados em um computador com processador Pentium Core 2 Duo a $2.66 \mathrm{GHz}$ e $2 \mathrm{~Gb}$ de RAM. A ferramenta TINA versão 2.9.2 foi usada na geração das classes de estado atômicas (Opção -A). O algoritmo que gera as classes de estados com restrições foi implementado na versão 0.60 do verificador. O programa fonte que gera as classes atômicas apresentado no artigo de Yoneda e Ryuba foi cedido pelo Dr. Tomohiro Yoneda, do National Institute of Informatics do Japão (a quem o autor deste trabalho agradece).

A tabela 5.5 mostra os resultados do experimento.

Os resultados mostram que dependendo do comportamento da rede, o ASCG de Berthomieu e o CSCG aqui proposto podem gerar grafos mais compactos: o 


\begin{tabular}{|l|c||c|c|c|}
\hline \multicolumn{2}{|c|}{ Exemplos } & Yoneda & ASCG & CSCG \\
\hline \multirow{3}{*}{ Fig. 5.12 (a) } & estados & 53 & 27 & 39 \\
& transições & 95 & 49 & 53 \\
& tempo de CPU(s) & 0.034 & 0.001 & 0.022 \\
\hline \multirow{3}{*}{ Fig. 5.12 (b) } & estados & 64 & 47 & 51 \\
& transições & 178 & 140 & 70 \\
& tempo de CPU(s) & 0.357 & 0.004 & 0.049 \\
\hline \multirow{3}{*}{ Fig. 5.12 (c) } & estados & 168 & 80 & 121 \\
& transições & 363 & 204 & 171 \\
& tempo de CPU(s) & 6.36 & 0.004 & 0.163 \\
\hline \multirow{2}{*}{ Fig. 5.12 (d) } & estados & 53 & 27 & 39 \\
& transições & 95 & 49 & 53 \\
& tempo de CPU(s) & 0.040 & 0.001 & 0.035 \\
\hline
\end{tabular}

Tabela 5.5: Resultados dos experimentos de Yoneda e Ryuba

CSCG gera grafos de menor tamanho apenas no caso $c$ enquanto o ASCG gera grafos menores nos casos $a, b$ e $d$ (o tamanho do grafo é dado pela soma dos estados e transições).

O ASCG não tem compromisso com a inclusão de todos os caminhos, e utiliza uma definição de equivalência menos restritiva ${ }^{1}$ portanto pode gerar grafos mais compactos. Isto pode ser notado pela tendência de gerar menos nós e mais arestas que o CSCG.

Note que o CSCG ganha do grafo das regiões geométricas em todos os quesitos o que deve-se em boa medida ao fato de desligar a seqüência de disparo da definição de classe de estados. Entretanto o ganho em eficiência deve-se de fato ao uso da propriedade de estabilidade no lugar da atomicidade como requisito para construir grafos que preservem as propriedades de tempo ramificado.

O ganho na performance no computo dos grafos do ASCG pode ser atribuída ao fato do CSCG ter sido implementado sobre as redes GHENeSys, o que implica em uma complexidade maior na determinação da habilitação, condição de disparo, tempo mínimo de habilitação etc por causa da inclusão do tempo nas marcas e pela necessidade de verificação da capacidade dos boxes.

O ganho significativo em performance entre o grafo proposto pelo Yoneda e o CSCG, apesar da complexidade da GHENeSys comparado às RPTs, devese ao fato do CSCG aplicar a restrição somente uma vez quando uma classe é avaliada como não estável, em lugar de continuar aplicando esta restrição a seus sucessores. Isto limitou também o número de operações de divisão e junção de

\footnotetext{
${ }^{1}$ No ASCG se um domínio temporal subsumes um outro de uma classe com igual marcação as classes de estados são fundidas em uma só.
} 
classes, e tornou o processo de detecção e aplicação da restrição em uma operação mais eficiente.

\subsection{Comparação dos métodos para verificação formal de sistemas}

Nesta seção apresenta-se uma comparação entre os métodos usados para a verificação do sistema de evaporação mostrado no capítulo anterior usando a ferramenta UPPAAL e GHENeSys agora dotada de um sistema de verificação formal.

A modelagem do sistema de evaporação em UPPAAL foi feita usando autômatos temporizados estendidos. A descrição do sistema consta de três partes: as declarações (globais e locais) os autômatos e a definição do sistema.

No anexo C é mostrada a modelagem feita com UPPAAL do sistema de evaporação usando a metodologia proposta em (MACHADO et al., 2007) e (SEABRA et al., 2007) - que também cederam os programas fonte feitos para o UPPAAL.

Antes de prosseguir é preciso sublinhar que o modelo do controlador, segundo modelado no módulo LADDER, não representa a realidade do sistema de evaporação. Nesse modelo não são considerados os casos das válvulas V3 e V4 estarem abertas ao mesmo tempo ou das válvulas V1, V2 e V3 estarem abertas ao mesmo tempo.

Outro destaque importante é que o tempo consumido no ciclo de controle do CLP é considerado no módulo LADDER como sendo entre 1 a 6 unidades de tempo, comparável ou mesmo inferior ao tempo em que o tanque 1 pode ficar cheio o que é uma situação irreal. Se isto acontecesse no sistema real, os tanques poderiam transbordar, o que pode ser visto comparando o tempo necessário para fazer o ciclo de controle e o tempo necessário para encher o tanque $1 \mathrm{com}$ as válvulas V1 e V2 abertas.

Certamente isto deveria conduzir ao resultado direto de que o estado overflow é atingível, e portanto a verificação da propriedade $\forall \square$ not T ANK1.over flow deveria dar um resultado positivo e não negativo (Ver figura C.5 do anexo). Provavelmente esta decisão de simplificar os casos de falha foi tomada para facilitar a modelagem e a verificação das propriedades das ações sobre as válvulas gerando ações que estão sincronizadas com o controlador, o que não representa o comportamento real do sistema. Veja a figura 5.13. 


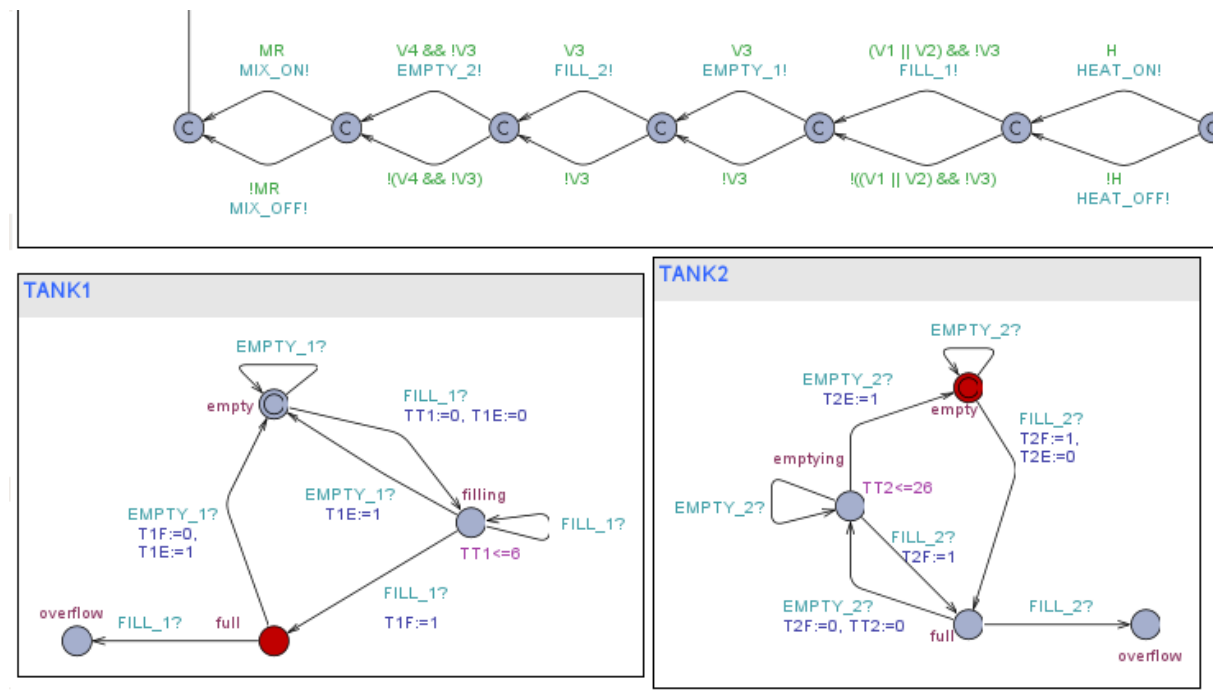

Figura 5.13: Exemplo de modelagem que conduz a erro na verificação de propriedades

A figura 5.13 mostra a parte do módulo LADDER onde as sinais do controlador são sincronizadas com os módulos que modelam o sistema além dos módulos TANK1 e TANK2. Note-se que mesmo quando o módulo TANK1 está no estado full este só pode passar ao estado overflow através de uma ordem do controlador, o que é modelado usando a sincronização FILL_1!. Na realidade a modelagem deveria ter sido feita seguindo o comportamento real qual seja: uma vez que o tanque 1 está cheio se a válvulas V1 e V2 não são fechadas a tempo o tanque 1 transborda.

O controlador só pode mudar as variáveis que estão no seu controle, e a modelagem da planta deve descrever o comportamento do sistema dependendo do estado destas variáveis, tal como foi feito na modelagem mostrada nas figuras 5.7 e 5.8. Veja que neste caso, se as válvulas V1 e V2 não são fechadas num tempo menor ou igual a 0.5 unidades de tempo a partir do instante que o tanque é considerado cheio este passará ao estado transbordando.

No artigo (MACHADO et al., 2007) assume-se que se estados onde as variáveis $X 1, \ldots X 13, X 21$ são computadas são atingíveis então está garantido o funcionamento correto do sistema, o que é falso. A partir da especificação em SFC do controlador (ver figura C.3 do anexo) pode-se assumir que a propriedade $\forall \square C O N D E N S E R . g o o d \rightarrow \forall \diamond A L A R M$.off será verdadeira.

Esta fórmula pode ser colocada em UPPAAL, da seguinte forma:

CONDENSER.good $\vee(T A N K 1 . e m p t y \wedge T A N K 2 . e m p t y) \rightarrow$ ALARM.off

a qual especifica que se o condensador funciona bem (o que é o mesmo que 
not Malf) ou os tanques 1 e 2 estão vazios e o alarme deve ser desligado.

Esta propriedade foi verificada sendo que a mesma não é satisfeita no modelo. (Ver figura C.5 do anexo). Também a propriedade que verifica a ausência de bloqueios falhou no processo de verificação.

O tempo usado pela ferramenta UPPAAL para dar os resultados foi o de aproximadamente 1 segundo para cada propriedade verificada o que é um resultado muito bom. Este resultado é decorrente das "aproximações" feitas na modelagem, de modo a aproveitar os recursos do ferramenta para diminuir o tempo de verificação.

O fato de colocar a maioria dos estados como "commited" reduz de forma dramática o espaço de estados, limitando a possibilidades de combinações de seqüencias através destes estados. Além disso, na modelagem foi usado um "recurso" para permitir que as atualizações das variáveis fossem feitas de uma vez só limitando assim o número de combinações possíveis.

Outro fato a considerar é que não foi possível verificar todas as propriedades que deveriam ser verificadas para determinar o correto funcionamento do sistema. A maioria das propriedades verificadas foram propriedades de segurança sendo verificada apenas duas entre as propriedades comportamentais ou de vivacidade. Isto pode ser constatado a partir da comparação da tabela 5.4 e a figura C.5.

Quanto ao método proposto, usado na verificação do sistema de evaporação no capítulo anterior, pode-se dizer que o modelo representa o comportamento do sistema e o seu controle de forma adequada. Todas as propriedades, tanto as de segurança quanto as comportamentais puderam ser verificadas. Como corresponde aos sistemas de tempo real foi possível determinar não apenas se a ação correta acontecia mas se esta acontecia no tempo determinado pela especificação.

No que se refere à performance o cálculo do espaço de estados consumiu um tempo maior que o usado na verificação com UPPAAL. Se diluído o cálculo do espaço de estados, na verificação das 16 propriedades - considerando o tempo usado pelos algoritmos de rotulação - podemos chegar a um estimado de tempo de 2 minutos para cada propriedade verificada. 


\section{Conclusões e Trabalhos Futuros}

Neste capítulo apresenta-se um resumo das principais contribuições deste trabalho assim como as conclusões e recomendações resultantes das abordagens introduzidas. Propostas para a continuidade do trabalho e para a geração de publicações também serão apresentadas.

\subsection{Conclusões}

Em qualquer método dedicado ao projeto de sistemas existem fases características - independentemente de como são articuladas ou superpostas - tais como as etapas de especificação, modelagem e verificação, necessárias para atingir o nível de segurança e qualidade desejados. Para sistemas temporizados, especificamente os de tempo real, existem poucas técnicas de verificação formal suficientemente eficientes para serem aplicadas a sistemas reais.

Neste trabalho foi proposta uma técnica que usa as RPTs como formalismo base para a modelagem de sistemas e uma abordagem enumerativa que garante a verificação de propriedades temporais definidas usando a TCTL. A vantagem principal desta abordagem é o uso da lógica TCTL como linguagem de especificação, a qual permite descrever propriedades qualitativas e quantitativas (estas últimas imprescindíveis para sistemas com restrições temporais) que incluem tanto as propriedades de segurança quanto as de vivacidade. Apenas a abordagem proposta em (ALUR; COURCOUBETIS; DILL, 1990) para autômatos temporizados e as RPT em (VIRBITSKAITE; POKOZY, 1999) possuem esta vantagem.

A grande diferença da técnica aqui proposta com relação a estas propostas é a eficiência do processo de verificação. Neste sentido dois resultados importantes foram atingidos: primeiro desvincular o cálculo do espaço de estados da fórmula que se deseja verificar, permitindo a verificação de várias fórmulas sem a necessidade de recomputar o espaço de estados do modelo; segundo, a melhoria do 
processo de geração do espaço de estados fazendo que o mesmo não dependa da magnitude das constantes de tempo do sistema de forma direta.

Especificamente, no que diz respeito aos algoritmos de geração do espaço de estados que preservam as propriedades de tempo ramificado, a proposta de uma nova condição suficiente para a preservação destas propriedades leva a partições melhores do espaço de estados e constitui uma contribuição para melhorar a eficiência na verificação das propriedades de vivacidade, geralmente associadas à funcionalidade.

A proposta de condições suficientes para realizar o cálculo correto dos incrementos de tempo e conseguir determinar a validade de propriedades quantitativas constitui outra contribuição do trabalho apresentado.

O desenvolvimento e implementação dos algoritmos de rotulação para o GTT, conjuntamente com a técnica de geração deste grafo constituíram a base do programa de verificação.

Na comparação com outras técnicas para o computo do espaço de estados o algoritmo aqui proposto mostrou-se superior ao apresentado em (YONEDA; RYUBA, 1998) em todos os parâmetros de comparação. Com relação a outros algoritmos disponíveis, os resultados dos experimentos mostraram-se similares em alguns parâmetros, sobretudo se considerado quando os algoritmos aqui implementados são aplicados sobre as redes GHENeSys. Também mostrou-se que estas outras técnicas não permitem a determinação de propriedades quantitativas de forma direta ${ }^{1}$ e as razões pelas quais este objetivo não pode ser atingido por elas, diferentemente da proposta deste trabalho.

Para lidar com o problema da explosão de estados, a rede GHENeSys foi estendida de forma a aceitar intervalos de tempo em lugar de durações fixas. Isto permitiu acoplar à rede a técnica de verificação desenvolvida para as RPT ao mesmo tempo que mantém os conceitos de hierarquia, abstração associada às estratégias top-down e bottom-up. A utilização combinada destas técnicas auxiliam o projeto de sistemas complexos e cria uma maior sinergia entre os processos de modelagem e verificação, típica dos projetos de sistemas reais.

A prova da manutenção das propriedades no processo de refinamento da rede - sejam nos sistemas seja nos sistemas temporizados - constitui a base para o uso de qualquer formalismo na modelagem, especificação e verificação de sistemas complexos. A rede GHENeSys mostrou-se neste sentido em plenas condições

\footnotetext{
${ }^{1}$ Sem o uso dos observadores.
} 
para ser utilizada no projeto deste tipo de sistema.

Também foi estabelecida a possibilidade de usar mecanismos de abstração e hierarquia para reduzir a complexidade dos algoritmos de verificação sempre que a propriedade a ser verificada assim o permita.

Através de um estudo de caso foi mostrada a utilidade dos pseudo-boxes como elemento de integração entre diferentes redes com um desempenho equivalente ao introduzido pelo uso de canais de sincronização nos autômatos temporizados (redes de autômatos).

\subsection{Trabalhos Futuros}

Neste trabalho foram considerados sistemas onde todos os intervalos de tempo têm limites diferentes de infinito. Também foi mostrado que a introdução de uma normalização não modifica os resultados de preservação de propriedades, mas seria interessante expandir o tipo de sistema que pode ser modelado e verificado usando a rede GHENeSys, ampliando o nível de indeterminação no disparo das transições.

O algoritmo de geração do GTT ainda pode ser melhorado, usando técnicas de redução de ordem parcial. Uma técnica como esta poderia melhorar a eficiência do processo de verificação através do cálculo de partições mais compactas do espaço de estados, sem comprometer as condições para o cálculo correto dos intervalos de tempo globais.

O próximo passo na evolução no processo de unificação das redes GHENeSys é introduzir tipos nas marcas - através de atributos de objetos - e com isso incluir a formulação das redes de alto nível. Desta forma, a rede atingiria o seu máximo potencial na representação de sistemas complexos se consideradas todas as extensões de redes disponíveis na comunidade acadêmica. Um estudo da conservação das propriedades das redes, assim como da manutenção das propriedades verificadas nos diferentes níveis de abstração, tornaria esta ferramenta o sistema indicado para o projeto de sistemas discretos ou pelo menos o primeiro sistema de redes unificadas implementado por completo.

Exsistem trabalhos reportados na literatura que combinam teoria de escalonamento com a abordagem da verificação de modelos tais como (BRABERMAN; FELDER, 1999), (HSIUNG; WANG; KUO, 1999), (WASZNIOWSKI; KRAKORA; HANZÁLEK, 2009). Baixo determinadas condições, a verificação formal pode ser usada também para o escalonamento de sistemas temporizados. Neste sentido 
seria necessário desenvolver novos algoritmos de busca no espaço de estados do modelo na procura de soluções ótimas ou pelo menos que satisfazam todos os requisitos do escalonador. Isto abre uma nova porta para estudos na direção da integração entre o ambiente GHENeSys e o ambiente para projetos de sistemas de planejamento itSIMPLE (Integrated Tools and Software Interface for Modeling PLanning Environments) (VAQUERO et al., 2007) vencedor da competição mundial entre sistemas deste tipo no ICKEPS 2009. 


\section{Referências}

AALST, W. Interval Timed Coloured Petri Nets and their Analysis. In: MARSAN, M. A. (Ed.). Application and Theory of Petri Nets 1993. [S.1.]: Springer-Verlag, Berlin, 1993. v. 691, p. 453-472.

ALUR, R.; COURCOUBETIS, C.; DILL, D. L. Model checking for real-time systems. In: Proceedings of the 5th IEEE Symposium on Logic in Computer Science. Piscataway, NJ: IEEE Press, 1990. p. 414-425.

ALUR, R.; COURCOUBETIS, C.; DILL, D. L. Model-checking in dense real-time. Information and Computation, v. 104, n. 1, p. 2-34, 1993.

AluR, R.; COURCOUBETIS, C.; HENZINGER, T. A.; HO, P.-H. Hybrid automata: An algorithmic approach to the specification and verification of hybrid systems. In: Hybrid Systems. [S.l.: s.n.], 1992. p. 209-229.

ALUR, R.; DILL, D. Automata for modeling real-time systems. Lecture Notes in Computer Science, v. 443, p. 322-335, 1990.

ALUR, R.; HENZINGER, T. Real-Time Logics: Complexity and Expressiveness. In: Fifth Annual IEEE Symposium on Logic in Computer Science. Washington, D.C.: IEEE Computer Society Press, 1990. p. 390-401.

ASARIN, E.; BOZGA, M.; KERBRAT, A.; MALER, O.; PNUELI, A.; RASSE, A. Data-structures for the verification of timed automata. In: Hybrid and Real-Time Systems. [S.l.]: Springer Verlag, 1997. v. 1201, p. 346-360.

BEHRMANN, G.; LARSEN, K. G.; PEARSON, J.; WEISE, C.; YI, W. Efficient timed reachability analysis using clock difference diagrams. In: Computer Aided Verification. Heidelberg, Germany: Springer Verlag, 1999. v. 1633, p. 341-353.

BERTHELOT, G. Occurrence graphs for interval timed coloured nets. In: VALETTE, R. (Ed.). Proceedings of ICATPN 94. [S.l.]: Springer Verlag, 1994. v. 815 , p. $79-98$.

BERTHOMIEU, B.; DIAZ, M. Modelling and verification of time dependent systems using time petri nets. IEEE Trans. on Software Engineering, v. 17, n. 3, p. 259-273, 1991.

BERTHOMIEU, B.; MENASCHE, M. An enumerative approach for analyzing time Petri nets. In: MASON, R. E. A. (Ed.). Information Processing: proceedings of the IFIP congress 1983. [S.l.]: Elsevier Science Publishers, Amsterdam, 1983. v. 9, p. 41-46.

BERTHOMIEU, B.; RIBET, P. O.; VERNADAT, F. The tool tina - construction of abstract state spaces for petri nets and time petri nets. Int. J. Prod. res., v. 42, n. 14, p. 2741-2756, July 2004. 
BERTHOMIEU, B.; VERNADAT, F. State class constructions for branching analysis of time petri nets. Lecture Notes in Computer Science, Springer-Verlag, v. 2619, p. 442-457, 2003.

BERTHOMIEU, B.; VERNADAT, F. State space abstractions for time petri nets. In: LEE, I.; LEUNG, J. Y.-T.; SON., S. H. (Ed.). Handbook of Real-Time and Embedded Systems. [S.1.]: Chapman and Hall/CRC, 2006.

BOUCHENEB, H.; ALGER, U.; BERTHELOT, G. Towards a simplified building of time petri nets reachability graph. In: Proc. 5th International Workshop on Petri Nets and Performance Models. Toulouse, France: [s.n.], 1993. p. $46-55$.

BOUCHENEB, H.; HADJIDJ, R. Using inclusion abstraction to construct atomic state class graphs for time petri nets. International Journal of Embedded Systems, v. 2, n. 1/2, p. 128-139, 2006.

BOUCHENEB, H.; MULLINS, J. Analyse des réseaux temporels. calcul des classes en o(n2) et des temps de chemin en o(m x n). Technique et Science Informatiques, v. 22, n. 4, p. 435-459, 2003.

BOUCHENEB, H.; RAKKAY, H. A more efficient time petri net state space abstraction preserving linear properties. acsd, IEEE Computer Society, Los Alamitos, CA, USA, v. 0, p. 61-70, 2007. ISSN 1550-4808.

BRABERMAN, V. A.; FELDER, M. Verification of real-time designs: Combining scheduling theory with automatic formal verification. In: NIERSTRASZ, O.; LEMOINE, M. (Ed.). ESEC/FSE '99. [S.1.]: Springer-Verlag, 1999. v. 1687, p. $494-510$.

BRYANT, R. E. Graph-based algorithms for boolean function manipulation. IEEE Trans. Comput., IEEE Computer Society, Washington, DC, USA, v. 35, n. 6, p. 677-691, 1986. ISSN 0018-9340.

BURCH, J.; CLARKE, E.; MCMILLAN, K.; DILL, D.; HWANG, L. Symbolic Model Checking: $10^{20}$ States and Beyond. In: Proceedings of the Fifth Annual IEEE Symposium on Logic in Computer Science. Washington, D.C.: IEEE Computer Society Press, 1990. p. 1-33.

CASSANDRAS, C. G. Discrete Event Systems: Modeling and Performance Analysis. [S.l.]: Aksen Associates Inc., 1993.

CERANS, K.; GODSKESEN, J. C.; LARSEN, K. G. Timed modal specifications - theory and tools. In: COURCOUBETIS, C. (Ed.). Proc. of 5th Computer-Aided Verification. [S.l.]: Springer Verlag, 1993. v. 697, p. 253-267.

CERONE, A.; MAGGIOLO-SCHETTINI, A. Time-based expressivity of time petri nets for system specification. Theoretical Computer Science, v. 216, n. 1, p. 1-53, March 1999.

Christensen, S.; KRISTEnSEN, L. M.; MAILUnD, T. Condensed state spaces for timed petri nets. In: COLOM, J. M.; KOUTNY, M. (Ed.). Proceedings of the Application and Theory of Petri Nets 2001. Berlin, Germany: Springer-Verlag, 2001. v. 2075, p. 101-120. 
CLARKE, E. M.; EMERSON, E. A. Design and synthesis of synchronization skeletons using branching time temporal logic. In: Proc. Workshop on Logics of Programs. Berlin: Springer Verlag, 1981. v. 131, p. 52-71.

CLARKE, E. M.; EMERSON, E. A.; SISTLA, A. P. Automatic verification of finite state concurrent systems using temporal logic specifications. ACM transactions on Programming Languages and Systems, v. 8, n. 2, p. 244-263, April 1986.

CLARKE, E. M.; WING, J. M.; ALUR, R.; CLEAVELAND, R.; DILL, D.; EMERSON, A.; GARLAND, S.; GERMAN, S.; GUTTAG, J.; HALL, A.; HENZINGER, T.; HOLZMANN, G.; JONES, C.; KURSHAN, R.; LEVESON, N.; MCMILlAN, K.; MOORE, J.; PELED, D.; PNUELI, A.; RUSHBY, J.; SHANKAR, N.; SIFAKIS, J.; SISTLA, P.; STEFFEN, B.; WOLPER, P.; WOODCOCK, J.; ZAVE, P. Formal methods: state of the art and future directions. ACM Computing Surveys, v. 28, n. 4, p. 626-643, 1996.

CORTES, L. A.; ELES, P.; PENG, Z. Modeling and formal verification of embedded systems based on a petri net representation. Journal of Systems Architecture, v. 49, p. 571-598, 2003.

DAMM, W.; JOSKO, B.; HUNGAR, H. A compositional real-time semantics of statemate designs. In: Compositionality: The Significant Difference. Heidelberg, Germany: Springer Verlag, 1998. v. 1536, p. 186-238.

DAVID, R.; ALLA, H. On hybrid petri nets. In: Discrete Event Dynamic Systems: Theory and Applications. [S.l.: s.n.], 2001. v. 11, n. 1/2, p. 9-40.

DAVID, R.; ALLA, H. Discrete, Continuous and Hybrid Petri Nets. [S.l.]: Springer-Verlag, 2005.

DAWS, C.; OLIVERO, A.; TRIPAKIS, S.; YOVINE, S. The tool KRONOS. In: Hybrid Systems III: Verification and Control. Rutgers University, New Brunswick, NJ, USA: Springer, 1995. v. 1066, p. 208-219.

DICESARE, F.; KULP, P. T.; GILE, M.; LIST, G. The application of petri nets to the modeling, analysis and control of intelligent urban traffic networks. In: Valette, R. (Ed.). Lecture Notes in Computer Science; Application and Theory of Petri Nets 1994, Proceedings 15th International Conference, Zaragoza, Spain. [S.l.]: Springer-Verlag, 1994. v. 815, p. 2-15.

DILL, D. L. Timing assumptions and verification of finite-state concurrent systems. In: Automatic Verification Methods for Finite State Systems. [S.l.: s.n.], 1989. p. 197-212.

EDER, J.; GRUBBER, W.; PANAGOS, E. Temporal modeling of workflows with conditional execution paths. In: DEXA 2000. [S.l.]: Springer Verlag, 2000. v. 1873 .

EDER, J.; PANAGOS, E.; RABINOVICH, M. Time constraints in workflow systems. In: CaiSE 99. [S.l.]: Springer Verlag, 1999. v. 1626, p. 323+.

EMERSON, E.; MOK, A.; SISTLA, A.; SRINIVASAN, J. Quantitative temporal reasoning. In: Proceedings of CAV'90. [S.l.]: Springer-Verlag, 1990. v. 531, p. $136-145$. 
EMERSON, E. A.; CLARKE, E. Characterizing correctness properties of parallel programs as fixpoints. In: ICALP 83: Automata, Languages, and Programming. [S.l.]: Springer Verlag, 1980. v. 85, p. 169-181.

EMERSON, E. A.; JUTLA, C. S.; SISTLA, A. P. On model-checking for fragments of $\mu$-calculus. In: $C A V$. [S.l.: s.n.], 1993. p. 385-396.

FOYO, P. M. G. del. GHENeSys: Uma Rede Estendida Orientada a Objetos para Projeto de Sistemas Discretos. Dissertação (Mestrado) — Escola Politecnica da Universidade de São Paulo, 2001.

FOYO, P. M. G. del; SILVA, J. R. Using time petri nets for modeling and verification of timed constrained workflow systems. In: Proceedings of the 19th International Congress of Mechanical Engineering. Brasilia, Brazil: ABCM, 2007.

FOYO, P. M. G. del; SILVA, J. R. The verification of real time systems using the tina tool. In: Proceedings of the IFAC World Congress. Seul, Korea: [s.n.], 2008.

GARDEY, G.; LIME, D.; MAGNIN, M.; ROUX, O. H. Romeo: A tool for analyzing time petri nets. In: ETESSAMI, K.; RAJAMANI, S. (Ed.). CAV2005. [S.l.]: Springer-Verlag, 2005. v. 3576, p. 418-423.

GHEZZI, C.; MANDRIOLI, D.; MORASCA, S.; PEZZE, M. A unified high-level petri net formalism for time-critical systems. IEEE Trans. on Software Engineering, IEEE Press, Piscataway, NJ, USA, v. 17, n. 2, p. 160-172, 1991. ISSN 0098-5589.

GIRAULT, C.; VALK, R. Petri Nets for System Engineering: A Guide to Modeling, Verification, and Applications. Secaucus, NJ, USA: Springer-Verlag New York, Inc., 2001. 597 p. ISBN 3540412174.

HADJIDJ, R.; BOUCHENEB, H. On-the-fly tctl model checking for time petri nets using state class graphs. acsd, IEEE Computer Society, Los Alamitos, CA, USA, v. 0, p. 111-122, 2006. ISSN 1550-4808.

HADJIDJ, R.; BOUCHENEB, H. Improving state class constructions for ctl* model checking of time petri nets. STTT, Springer Verlag, Los Alamitos, CA, USA, v. 10, n. 2, p. 167-184, 2008.

HAREL, D. Statecharts: A visual formalism for complex systems. Science of Computer Programming, v. 8, n. 3, p. 231-274, June 1987.

HELJANKO, K. Model Checking the Branching Time Temporal Logic CTL. [S.l.], 1997.

HENZINGER, T.; NICOLLIN, X.; SIFAKIS, J.; YOVINE, S. Symbolic Model Checking for Real-Time Systems. In: 7th. Symposium of Logics in Computer Science. Santa-Cruz, California: IEEE Computer Scienty Press, 1992. p. 394-406.

HENZINGER, T. A.; HO, P.-H.; WONG-TOI, H. HYTECH: A model checker for hybrid systems. International Journal on Software Tools for Technology Transfer, v. 1, n. 1-2, p. 110-122, 1997. 
HENZINGER, T. A.; KOPKE, P. W.; PURI, A.; VARAIYA, P. What's decidable about hybrid automata? In: Proc. 27th Annual ACM Symp. on Theory of Computing (STOC). [S.l.: s.n.], 1995. p. 373-382.

HENZINGER, T. A.; KUPFERMAN, O.; VARDI, M. Y. A space-efficient on-th-fly algorithm for real-time model-checking. In: CONCUR'96. Heidelberg, Germany: Springer Verlag, 1996. v. 1119, p. 514-529.

HOARE, C. A. R. Communicating Sequential Processes. Englewood Cliffs, NJ: Prentice-Hall, 1985.

HSIUNG, P.-A.; WANG, F.; KUO, Y.-S. Scheduling system verification. Lecture Notes in Computer Science, v. 1579, p. 19-33, 1999.

HUUCK, R.; LUKOSCHUS, B.; LAKHNECH., Y. Verifying untimed and timed aspects of the experimental batch plant. In: Special Issue: Verification of Hybrid Systems - Results of a European Union Esprit Project. [S.1.]: Hermes Science Publishing, 2001. v. 7, n. 4, p. 400-415.

JAHANIAN, F.; MOK, A. K. Safety analysis of timing properties in real-time systems. IEEE Trans. on Software Engineering, IEEE Press, Piscataway, NJ, USA, v. 12, n. 9, p. 890-904, 1986. ISSN 0098-5589.

JAHANIAN, F.; MOK, A. K. Modechart: a specification language for real-time systems. IEEE Trans. on Software Engineering, v. 20, p. 933-947, Dec 1994.

JENSEN, K. Coloured Petri Nets: basic concepts, analysis methods and practical use. Berlin: Springer, 1992. (Monographs in Theoretical Computer Science, v. 1: Basic Concepts).

J.MøLLER; LICHTENBERG, J.; ANDERSEN, H. R.; HULGAARD, H. Difference decision diagrams. In: Annual Conf. Eur. Assoc. Computer Science Logic (CSL). Madrid, Spain: [s.n.], 1999.

JONES, N. D.; LANDWEBER, L. H.; LIEN, Y. E. Complexity of some problems in petri nets. Theor. Comput. Sci., v. 4, n. 3, p. 277-299, 1977.

KOWALEWSKI, S.; STURSBERG, O.; BAUER, N. An experimental batch plant as a test case for the verification of hybrid systems. European Journal of Control, v. 7, n. 4, p. 366-381, 2001.

LAKOS, C.; PETRUCCI, L. Modular state space exploration for timed petri nets. STTT, v. 9, n. 3-4, p. 393-411, 2007.

LAMPORT, L. Proving the correctness of multiprocess programs. IEEE Trans. on Software Engineering, v. 3, n. 2, p. 125-143, March 1977.

LAROUSSINIE, F.; LARSEN, K. G. CMC: A tool for compositional model-checking of real-time systems. In: Proc. IFIP Joint Int. Conf. Formal Description Techniques \& Protocol Specification, Testing, and Verification (FORTE-PSTV'98), Paris, France, Nov. 1998. [S.l.]: Kluwer Academic, 1998. p. $439-456$.

LARSEN, K. G.; PETTERSSON, P.; YI, W. UPPAAL - Validation and Verification of Real Time Systems - Status $\&$ Developments. 
LARSEN, K. G.; PETTERSSON, P.; YI, W. Compositional and symbolic model-checking of real-time systems. In: IEEE Real-Time Systems Symposium. [S.l.: s.n.], 1995. p. 76-89.

LIME, D.; ROUX, O. H. Model checking of time petri nets using the state class timed automaton. Discrete Event Dyn Syst, v. 16, p. 179-206, 2006.

MACHADO, J. M.; CAMPOS, J. C.; SOARES, F.; LEãO, C. P.; SILVA, J. C. L. F. da. Simulation and formal verification of industrial systems controllers. In: Proceedings of the 19th International Congress of Mechanical Engineering. Brasilia, Brazil: ABCM, 2007.

MERLIN, P.; FABER, D. Recoverability of communication protocolsimplications of a theoretical study. Communications, IEEE Transactions on [legacy, pre-1988], v. 24, n. 9, p. 1036-1043, Sep 1976.

MILNER, A. A calculus of communicating systems. Lecture Notes in Computer Science, v. 92, p. 1, 1980.

MIYAGI, P. E. Control System Design, Analysis and Implementation of Discrete Event Production Systems by using Mark Flow Graph. Tese (Doutorado) Tokyo Institute of Technology, Tokyo, 1988.

PAGANI, F. Partial orders and verification of real-time systems. In: Formal Techniques in Real-Time and Fault-Tolerant Systems. Heidelberg, Germany: Springer Verlag, 1996. v. 1135, p. 327-346.

PENCZEK, W.; PÓŁROLA, A. Abstractions and partial order reductions for checking branching properties of time Petri nets. Lecture Notes in Computer Science, v. 2075, p. 323+, 2001.

PNUELI, A. The temporal logic of programs. In: FOCS. [S.l.: s.n.], 1977. p. 46-57.

RAMCHANDANI, C. Analysis of asynchronous concurrent systems by timed Petri Nets. Cambridge, MA, USA, 1974.

REED, G. M.; ROSCOE, A. W. A timed model for communicating sequential processes. Theor. Comput. Sci., v. 58, p. 249-261, 1988.

RIVIERE, N.; VALETTE, R.; PRADIN-CHEZALVIEL, B.; UPS, I. A. . Reachability and temporal conflicts in t-time petri nets. In: PNPM '01: Proceedings of the 9th international Workshop on Petri Nets and Performance Models (PNPM'01). Washington, DC, USA: IEEE Computer Society, 2001. p. 229.

ROUX, O. H.; DéPLANCHE, A. M. A t-time petri net extension for real time-task scheduling modeling. European Journal of Automation, v. 36, p. 973-987, 2002.

SCHNEIDER, S. Concurrent and Real-Time Systems: The CSP Approach. New York: Wiley, 1999.

SCHWOON, S.; ESPARZA, J. A note on on-the-fly verification algorithms. In: Tools and Algorithms for the Construction and Analysis of Systems. [S.1.]: Springer Berlin / Heidelberg, 2005. v. 3440, p. 174-190. 
SEABRA, E.; MACHADO, J.; SILVA, J. F. da; SOARES, F. O.; LEãO, C. P. Simulation and formal verification of real time systems: A case study. In: ICINCO 200\%, Proceedings of the Fourth International Conference on Informatics in Control, Automation and Robotics, Signal Processing, Systems Modeling and Control. Angers, France: [s.n.], 2007.

SHAW, A. Communicating real-time state machines. IEEE Trans. on Software Engineering, v. 18, p. 805-816, Sept 1992.

SIFAKIS, J. Performance evaluation of systems using nets. In: Proceedings of the Advanced Course on General Net Theory of Processes and Systems. London, UK: Springer-Verlag, 1980. p. 307-319. ISBN 3-540-10001-6.

SIFAKIS, J. A unified approach for studying the properties of transition systems. Theoretical Computer Science, v. 18, p. 227-258, 1982.

SILVA, J. R. Interactive design of integrated systems. In: MATOS, L. C.; AFSARMANESH, H. (Ed.). Intelligent Systems for Manufacturing. [S.l.]: Kluwer Academic Pub., 1998.

SILVA, J. R.; MIRAlleS, J. A. S. P.; SALMON, A. O.; FOYO, P. M. G. del. Introducing object-orientation in unified petri net approach. In: Proceedings of the 20th International Congress of Mechanical Engineering. Gramado, Brazil: ABCM, 2009.

SILVA, J. R.; MIYAGI, P. E. PFS/MFG: A high level net for the modeling of discrete manufacturing systems. In: MATOS, L. C.; AFSARMANESH, H. (Ed.). Balanced Automation Systems, Arquitectures and Design Methods. [S.l.]: Chapman \& Hall, 1995. p. 349-362.

SILVA, J. R.; MIYAGI, P. E. A formal approach to pfs/mfg: a petri net representation of discrete manufacturing systems. In: Studies in Informatics and Control. Romenia: IC Publications, 1996.

SILVA, J. R.; PESSOA, F. J. B. Análise semiautomática de mark flow graph. In: Ibero-American Workshop in Autonomous Systems Robotics and CIM. Lisbon: [s.n.], 1992.

SUZUKI, I.; MURATA, T. Stepwise refinements of transitions and places. Lecture Notes in Computer Science, v. 52, p. 136-141, 1982.

TORRE, S. L.; NAPOLI, M. A decidable dense branching-time temporal logic. Lecture Notes in Computer Science, v. 1974, p. 139-151, 2000.

TOUSSAINT, J.; SIMONOT-LION, F.; THOMESSE, J. P. Time constraints verification methods based on time petri nets. In: Proc. of the 6th IEEE Workshop on Future Trends of Distributed Computing Systems (FTDCS '97). Washington, DC, USA: IEEE Computer Society, 1997. p. 262-.

TSAI, J. J. P.; YANG, S. J.; CHANG, Y.-H. Timing constraint petri nets and their application to schedulability analysis of real-time system specifications. IEEE Trans. on Software Engineering, IEEE Press, Piscataway, NJ, USA, v. 21, n. 1, p. 32-49, 1995. ISSN 0098-5589.

VALETTE, R. Analysis of petri nets by stepwise refinement. Journal of Computer Science, v. 18, p. 35-46, 1979. 
VAQUERO, T. S.; ROMERO, V.; TONIDANDEL, F.; SILVA, J. R. itsimple 2.0: An integrated tool for designing planning domains. In: 18th International Conference on Automated Planning and Scheduling. Providence, Rhode Island: [s.n.], 2007.

VERGAUWEN, B.; LEWI, J. A linear model checking algorithm for ctl. In: BEST, E. (Ed.). Proceedings of 4 th International Conference on Concurrency Theory. [S.l.]: Springer Verlag, 1993. v. 715, p. 447-461.

VIRBITSKAITE, I.; POKOZY, E. A partial order method for the verification of time petri nets. In: CIOBANU, G.; PAUN, G. (Ed.). FCT. [S.l.]: Springer Verlag, 1999. v. 1684, p. 547-558.

WANG, F. Red: Model-checker for timed automata with clock-restriction diagram. In: DEPT. OF INFORMATION TECHNOLOGY, UPPSALA UNIV. Proc. Workshop Real-Time Tools. [S.l.]: Technical Report 2001-014, 2001.

WANG, F. Symbolic verification of complex real-time systems with clockrestriction diagram. In: IFIP Int. Conf. Formal Techniques for Networked and Distributed Systems. Cheju Island, Korea: [s.n.], 2001.

WANG, F. Formal verification of timed systems: A survey and perspective. Proceedings of the IEEE, v. 92, n. 8, p. 1283-1305, August 2004.

WANG, F.; MOK, A. K.; EMERSON, E. A. Formal specification of synchronous distributed real-time systems by aptl. In: ICSE. [S.l.: s.n.], 1992. p. 188-198.

WANG, J.; DENG, Y.; XU, G. Reachability analysis of real-time systems using time petri nets. IEEE Transactions on Systems, Man and Cybernetics, v. 30, n. 5, p. 725-736, October 2000.

WASZNIOWSKI, L.; KRAKORA, J.; HANZÁLEK, Z. Case study on distributed and fault tolerant system modeling based on timed automata. Journal of Systems and Software, v. 82, n. 10, p. 1678-1694, 2009.

WFMC. Workflow Management Coalition Terminology and Glossary. Brussels: [s.n.], 1996.

YONEDA, T.; RYUBA, H. Ctl model checking of time petri nets using geometric regions. IEICE Trans. on Information and Systems, E81-D, n. 3, p. 297-396, 1998.

YONEDA, T.; SCHLINGLOFF, B.-H. Efficient verification of parallel real-time systems. In: Formal Methods in System Design. [S.l.: s.n.], 1997. v. 11, p. $187-215$.

YOVINE, S. Model checking timed automata. In: European Educational Forum: School on Embedded Systems. [S.l.: s.n.], 1996. p. 114-152.

ZUBEREK, W. M. Timed petri nets and preliminary performance evaluation. In: ISCA '80: Proceedings of the 7th annual symposium on Computer Architecture. New York, NY, USA: ACM Press, 1980. p. 88-96. 


\section{Apêndice A - Resultados da ferramenta TINA}

\begin{tabular}{|c|c|c|}
\hline Classe de estado & Marcação & Domínio temporal \\
\hline class 0 & $\mathrm{p} 1 \mathrm{p} 6$ & $\begin{array}{l}0 \leq t 1 \leq 1 \\
5 \leq t 5 \leq 6\end{array}$ \\
\hline class 1 & $\mathrm{p} 2 \mathrm{p} 6$ & $\begin{array}{l}0 \leq t 2 \leq 1 \\
4 \leq t 5 \leq 6\end{array}$ \\
\hline class 2 & $\mathrm{p} 3 \mathrm{p} 6$ & $0 \leq t 3 \leq 1$ \\
& & $3 \leq t 5<6$ \\
\hline class 3 & $\mathrm{p} 4 \mathrm{p} 6$ & $1 \leq t 4 \leq 3$ \\
& & $2 \leq t 5 \leq 6$ \\
\hline class 4 & $\mathrm{p} 5 \mathrm{p} 6$ & $0 \leq t 5 \leq 5$ \\
\hline class 5 & $\mathrm{p} 5 \mathrm{p} 7$ & \\
\hline class 6 & $\mathrm{p} 4 \mathrm{p} 7$ & $0 \leq t 4 \leq 1$ \\
\hline
\end{tabular}

Tabela A.1: Classes de estado lineares obtidas usando a opção -W para o exemplo da figura 3.2 


\begin{tabular}{|c|c|c|}
\hline Classe de estado & Marcação & Domínio temporal \\
\hline class 0 & $\mathrm{p} 1 \mathrm{p} 6$ & $\begin{array}{l}0 \leq t 1 \leq 0 \\
0 \leq t 5 \leq 0\end{array}$ \\
\hline & & $\begin{array}{l}0 \leq t 2 \leq 0 \\
1 \leq t 5 \leq 1\end{array}$ \\
\hline class 1 & $\mathrm{p} 2 \mathrm{p} 6$ & $0 \leq t 2 \leq 0$ \\
& $\mathrm{p} 2 \mathrm{p} 6$ & $0 \leq t 5<1$ \\
\hline class 2 & $\mathrm{p} 3 \mathrm{p} 6$ & $0 \leq t 3 \leq 0$ \\
& & $0 \leq t 5<2$ \\
\hline class 3 & $\mathrm{p} 3 \mathrm{p} 6$ & $2 \leq t 5 \leq 2$ \\
\hline class 4 & $\mathrm{p} 3 \mathrm{p} 6$ & $0 \leq t 3 \leq 0$ \\
& & $0 \leq t 5<1$ \\
\hline class 5 & $\mathrm{p} 4 \mathrm{p} 6$ & $0 \leq t 4 \leq 0$ \\
& & $2 \leq t 5<3$ \\
\hline class 6 & $\mathrm{p} 4 \mathrm{p} 6$ & $0 \leq t 4 \leq 0$ \\
& & $0 \leq t 5<2$ \\
\hline class 7 & $\mathrm{p} 5 \mathrm{p} 6$ & $1 \leq t 5 \leq 6$ \\
\hline class 8 & $\mathrm{p} 4 \mathrm{p} 7$ & $2 \leq t 4 \leq 3$ \\
\hline class 9 & $\mathrm{p} 5 \mathrm{p} 7$ & \\
\hline class 10 & & \\
\hline
\end{tabular}

Tabela A.2: Classes de estado atômicas obtidas usando a opção -A para o exemplo da figura 3.2

\section{A.1 Cálculo dos intervalos de tempo para o grafo das classes de estado atômicas}

A seguir mostra-se a aplicação do algoritmo proposto em (FOYO; SILVA, 2008) para calcular os incrementos de tempo no grafo das classes de estado atômicas.

$$
\begin{aligned}
& C_{0} \stackrel{t_{1}}{\longrightarrow} C_{1} \quad \text { class } 0 \quad \text { class } 1 \quad C_{0} \stackrel{t_{1}}{\longrightarrow} C_{2} \quad \text { class } 0 \quad \text { class } 2 \\
& \rightarrow 0 \leq t_{1} \leq 0 \quad 0 \leq t_{1} \leq 1 \quad \rightarrow \quad 0 \leq t_{1} \leq 0 \quad 0 \leq t_{1} \leq 1 \\
& 0 \leq t_{5} \leq 0 \quad 1 \leq t_{5} \leq 1 \quad 0 \leq t_{5} \leq 0 \quad 0 \leq t_{5}<1 \\
& {[1,1]} \\
& {[0,1]} \\
& \begin{array}{rll}
C_{1} \stackrel{t_{2}}{\longrightarrow} C_{3} & \text { class } 1 & \text { class } 3 \\
\rightarrow & 0 \leq t_{2} \leq 0 & 0 \leq t_{2} \leq 1 \\
& 1 \leq t_{5} \leq 1 & 1 \leq t_{5}<2
\end{array} \\
& {[0,1]} \\
& C_{1} \stackrel{t_{2}}{\longrightarrow} C_{4} \quad \text { class } 1 \quad \text { class } 4 \\
& \rightarrow 0 \leq t_{2} \leq 0 \quad 0 \leq t_{2} \leq 1 \\
& 1 \leq t_{5} \leq 1 \quad 2 \leq t_{5} \leq 2 \\
& {[1,1]} \\
& C_{2} \stackrel{t_{2}}{\longrightarrow} C_{3} \quad \text { class } 2 \quad \text { class } 3 \\
& \rightarrow 0 \leq t_{2} \leq 0 \quad 0 \leq t_{2} \leq 1 \\
& 0 \leq t_{5}<1 \quad 1 \leq t_{5}<2 \\
& {[1,1]} \\
& \begin{array}{rll}
C_{3} \stackrel{t_{3}}{\longrightarrow} C_{6} & \text { class } 3 & \text { class } 6 \\
\rightarrow & 0 \leq t_{3} \leq 0 & 0 \leq t_{3} \leq 1 \\
& 1 \leq t_{5}<2 & 2 \leq t_{5}<3
\end{array} \\
& \begin{array}{rll}
C_{2} \stackrel{t_{2}}{\longrightarrow} C_{5} & \text { class } 2 & \text { class } 5 \\
\rightarrow & 0 \leq t_{2} \leq 0 & 0 \leq t_{2} \leq 1 \\
& 0 \leq t_{5}<1 & 0 \leq t_{5}<1 \\
{[0,0]} & &
\end{array} \\
& C_{3} \stackrel{t_{3}}{\longrightarrow} C_{7} \quad \text { class } 3 \quad \text { class } 7 \\
& \rightarrow 0 \leq t_{3} \leq 0 \quad 0 \leq t_{3} \leq 1 \\
& 1 \leq t_{5}<2 \quad 0 \leq t_{5}<2 \\
& {[0,0]}
\end{aligned}
$$




$$
\begin{aligned}
& C_{4} \stackrel{t_{3}}{\longrightarrow} C_{6} \quad \text { class } 4 \quad \text { class } 6 \quad C_{5} \stackrel{t_{3}}{\longrightarrow} C_{7} \quad \text { class } 5 \quad \text { class } 7 \\
& \rightarrow 0 \leq t_{3} \leq 0 \quad 0 \leq t_{3} \leq 1 \\
& \rightarrow 0 \leq t_{3} \leq 0 \quad 0 \leq t_{3} \leq 1 \\
& 2 \leq t_{5} \leq 2 \quad 2 \leq t_{5} \leq 3 \\
& {[0,1]} \\
& {[0,1]} \\
& C_{6} \stackrel{t_{4}}{\longrightarrow} C_{8} \quad \text { class } 6 \quad \text { class } 8 \quad C_{6} \stackrel{t_{5}}{\longrightarrow} C_{9} \quad \text { class } 6 \quad \text { class } 9 \\
& \rightarrow \quad 0 \leq t_{4} \leq 0 \quad 1 \leq t_{4} \leq 3 \\
& 2 \leq t_{5} \leq 3 \quad 1 \leq t_{5} \leq 6 \\
& {[1,3]} \\
& \rightarrow 2 \leq t_{5} \leq 3 \quad 5 \leq t_{5} \leq 6 \\
& {[3,3]} \\
& C_{7} \stackrel{t_{4}}{\longrightarrow} C_{8} \quad \text { class } 7 \quad \text { class } 8 \quad C_{8} \stackrel{t_{5}}{\longrightarrow} C_{10} \quad \text { class } 8 \quad \text { class } 10
\end{aligned}
$$

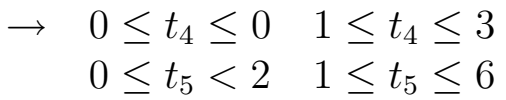

$$
\begin{aligned}
& {[1,3]} \\
& C_{9} \stackrel{t_{4}}{\longrightarrow} C_{10} \quad \text { class } 9 \quad \text { class } 10 \\
& \rightarrow 2 \leq t_{4} \leq 3 \quad 1 \leq t_{4} \leq 3 \\
& {[0,0]} \\
& \rightarrow \quad 1 \leq t_{5} \leq 6 \quad 5 \leq t_{5} \leq 6 \\
& {[4,0]}
\end{aligned}
$$




\section{Apêndice B - Provas}

\section{B.1 Prova de correção do algoritmo AUcheck}

Prova Para garantir que o algoritmo que rotula os estados que satisfazem a fórmula com operador UNTIL com o quantificador universal devemos mostrar que ao concluir a execução do algoritmo a seguinte fórmula é válida:

$$
\forall s\left[\left(\forall\left[p U_{\sim_{c}} q\right] \in L(s)\right) \leftrightarrow s \models \forall\left[p U_{\sim_{c}} q\right]\right]
$$

Isto será mostrado aplicando o princípio de indução na profundidade das chamadas recursivas ao procedimento DFS e garantindo que todos os estados da estrutura foram visitados pelo algoritmo.

Seja $\{I\}$ a junção das seguintes asserções:

I1 Todos os estados estão corretamente rotulados com as subfórmulas $p$ e $q$ : $\forall s[(p \in L(s)) \leftrightarrow s \models p \wedge(q \in L(s)) \leftrightarrow s \models q]$

I2 Se o stack não está vazio, então os estados alocados no stack formam um caminho no GTT:

$\forall i[1 \leq i<$ length $(\operatorname{stack}) \rightarrow(\operatorname{stack}(i), \operatorname{stack}(i+1)) \in E]$.

I3 O estado passado como parâmetro ao procedimento DFS é um sucessor do estado no topo do stack:

$($ Top $($ stack $), s) \in E$.

I4 $p \wedge \neg q$ vale para cada estado alocado no stack:

$\forall i[1 \leq i<$ length $($ stack $) \leftrightarrow s \models p \wedge \neg q]$.

I5 Cada estado alocado no stack foi visitado mas não rotulado:

$\forall i\left[1 \leq i<\right.$ length $($ stack $) \leftrightarrow$ state $[\operatorname{stack}(i)]$.visited $\wedge\left(\forall\left[p U_{\sim c} q\right] \notin L(s)\right)$

I6 Se um estado está rotulado com $\forall\left[p U_{\sim_{c} q}\right]$ então o mesmo foi visitado e $\forall\left[p U_{\sim_{c}} q\right]$ é válido nesse estado:

$\forall s\left[\right.$ out $\leftrightarrow$ state $[s]$.visited $\wedge s \models \forall\left[p U_{\left.\left.\sim_{c} q\right]\right]}\right.$.

I7 Se um estado foi visitado mas não foi rotulado com $\forall\left[p U_{\sim_{c}} q\right]$ nem está alocado no stack então $\forall\left[p U_{\sim_{c}} q\right]$ não é válida nesse estado:

$\forall s\left[\left(\forall\left[p U_{\left.\sim_{c} q\right]} \notin L(s)\right) \wedge\right.\right.$ state $[s]$.visited $\wedge \neg \exists i[1 \leq i<$ length $($ stack $) \wedge s=$ $\left.\operatorname{stack}(i)] \leftrightarrow s \models \neg \forall\left[p U_{\sim c} q\right]\right]$. 
I8 O conteúdo do stack antes da primeira chamada ao procedimento $D F S$ deve ser igual ao conteúdo do mesmo ao concluir as chamadas recursivas ao mesmo:

stack $_{0}=$ stack

I9 Todo estado visitado atualiza os tempos mínimos e máximos em que foi atingido. Seja $\left\lceil\lambda_{s}\right\rceil$ o conjunto de todos os caminhos que começam no estado inicial $s_{0}$ e terminam em $s$.

$\forall s\left[\right.$ state $[s]$. visited $\leftrightarrow \forall \sigma \in\left\lceil\lambda_{s}\right\rceil$ state $[s]$. tmin $\leq t_{\sigma} \leq$ state $\left.[s] . t m a x\right]$.

I10 Todos os estados rotulados com $\forall\left[p U_{\sim_{c}} q\right]$ atualizam os contadores qmin e qmax com os mínimos e máximos valores até atingir um estado em que $q$ é verdadeiro. Seja $\left\lceil\lambda_{q}\right\rceil$ o conjunto de todos os caminhos que começam no estado $s$ e atingem pela primeira vez um estado onde $q$ é verdadeiro. $\forall s\left[\forall\left[p U_{\sim c} q\right] \in L(s)\right] \leftrightarrow \forall \sigma \in\left\lceil\lambda_{q}\right\rceil$ state $[s]$. qmin $\leq t_{\sigma} \leq$ state $\left.[s] . q \max \right]$.

I11 Todos os estados que satisfazem out e TcheckB() ou out e $q$ satisfazem $\forall\left[p U_{\sim_{c}} q\right]:$ $\forall s\left[(\right.$ out $\wedge T$ checkB ()$) \vee(q \in L(s)) \leftrightarrow \forall\left[p U_{\sim c} q\right] \in L(s) \wedge$ out $]$.

A hipóteses indutiva que precisa ser provada é a seguinte:

$$
\{I\} \quad D F S() \quad\left\{I \wedge[\text { out } \wedge T \operatorname{check} B()] \vee[q \in L(s)] \leftrightarrow s \models \forall\left[p U_{\sim_{c} q} q\right\}\right.
$$

Antes de chamar o procedimento DFS todos os estados aparecem como não visitados $(\forall s$ state $[s] . v i s i t e d=$ false $)$, o stack está vazio, e nenhum estado está rotulado com $\forall\left[p U_{\sim_{c} q}\right]$. Considerando que todos os estados estão corretamente rotulados com as subfórmulas $p$ e $q$ então podemos garantir que o conjunto de hipóteses de indução $\{I\}$ é válido antes da primeira chamada a $D F S$.

Como o procedimento DFS não modifica em momento algum os rótulos $p \mathrm{e}$ $q$ nos estados, consideramos que $I 1$ é válida em qualquer situação se e somente se a mesma é válida antes de chamar DFS pela primeira vez. Sendo assim, I1 se mantém válida durante as chamadas a $D F S$ e portanto é válida quando todas as chamadas foram executadas.

A seguir vamos a analisar a validade das hipóteses de indução durante a execução das chamadas a $D F S$.

Case 1 Suponha que $\neg p \wedge \neg q$ é válida em um estado $s$ passado como parâmetro a DFS. DFS marca o estado $s$ como visitado, e coloca a variável out em falso, executa o procedimento UpdateList(s) para colocar em List os sucessores de $s$ que ainda não foram visitados e atualiza os relógios dos estados sucessores de $s$ com o tempo mínimo e máximo para atingir esses estados a partir de $s^{1}$ e retorna. Devido a que $\{I\}$ é válida antes da execução de $D F S$, o stack não foi modificado e $\forall\left[p U_{\sim_{c}} q\right]$ é falso em $s$, a ação correta foi realizada (out $=$ false e $\forall\left[p U_{\sim_{c}} q\right] \notin L(s)$ ) e $\{I\}$ se mantém válida logo da execução de $D F S$ nestas condições.

Case 2 Suponha que $p \wedge \neg q$ é válida num estado $s$ passado como parâmetro a $D F S$ e existem $n$ estados no stack: $\left(\right.$ length $($ stack $\left.)=n, n \in N^{+}, n \leq|S|\right)$.

\footnotetext{
${ }^{1}$ isto resulta importante para provar que o procedimento $D F S$ procura em todo o espaço de estado
} 
Nesse caso DFS marca o estado $s$ como visitado, atualiza os contadores de tempo (I9 é válida), coloca $s$ no stack (I4, I5 são válidas) e chama $D F S$ de forma recursiva para cada sucessor $s_{i}{ }^{2}$ de $s$ (I3 é válida) ${ }^{3}$. Como I2 é válida antes da chamada de $D F S$, os estados no stack formam um caminho de $n$ estados no GTT terminando num estado do qual $s$ é sucessor, então, depois da execução de DFS os estados no stack formam um caminho de $n+1$ estados terminando em $s$ (I2 é válida).

Ao retornar das chamadas do DFS para os sucessores do estado $s_{i}$, suponha que a fórmula foi avaliada como verdadeira e os contadores foram devidamente atualizados ${ }^{4}$. Os contadores para o estado $s_{i}$ contém os tempos máximos e mínimos para atingir um estado no qual a proposição $q$ é válida (I10 é valida).

O estado $s_{i}$ é retirado do stack levando este ao mesmo estado que se encontrava antes da chamada a $D F S$ (I9 é válida). A restrição temporal $\sim c$ é verificada através de $T$ check $B$ e, se é satisfeita o estado $s_{i}$ é rotulado com $\forall\left[p U_{\left.\sim_{c} q\right]}\right]$ e o procedimento retorna verdadeiro. Se a verificação da restrição temporal falha, então a fórmula não é válida nesse estado, portanto o mesmo não é rotulado e o procedimento retorna verdadeiro (I6, I7 e I11 são válidos).

Agora suponha que a fórmula falhou em pelo menos um estado sucessor de $s_{i}$. Sendo assim, a fórmula não é válida em $s_{i}$ segundo a condição 3.16. Neste caso o estado é retirado do stack sem rotular e o procedimento retorna falso (I10, I11, I6 e I7 são válidos). Portanto o conjunto das hipóteses $\{I\}$ são válidas depois da execução de $D F S$.

Case 3 Suponha que a proposição $q$ é válida num estado $s$ passado como parâmetro ao procedimento DFS. Nesse caso DFS marca o estado $s$ como visitado, os contadores cmin, cmax, qmin e qmax atualizam o tempo em que o estado foi atingido (I9 e I10 são válidas) e o estado é rotulado com a fórmula $\forall\left[p U_{\sim_{c} q}\right]^{5}$. O procedimento retorna verdadeiro (I6 e I11 são válidas).

Independente da validade da fórmula o procedimento UpdateList(s) é executado para colocar os sucessores de $s$ que não foram visitados na lista de estados a verificar.

Como $\{I\}$ é válida antes da execução de $D F S$ e o stack não foi modificado as hipóteses de indução I2, I3, I4, I5 e I8 se mantém válidas. Sendo assim, o conjunto das hipóteses $\{I\}$ é válida depois da execução de $D F S$.

Case 4 Suponha que o estado $s$ foi visitado. Isto só pode acontecer de forma recursiva pois $A U C h e c k$ só executa estados colocados na lista os quais não têm sido visitados.

Como o estado já foi visitado e $\{I\}$ é válida antes da execução de $D F S$, o estado $s$ já está rotulado com a fórmula se e so se a mesma é válida nesse estado. Se o estado está rotulado com $\forall\left[p U_{\sim_{c}} q\right]$ então o procedimento retorna verdadeiro e os valores de qmin e qmax atualizados com os tempos

\footnotetext{
${ }^{2} i \in N, i \leq|R(s)|$

3 a diferença do algoritmo DFS com seus similares em (CLARKE; EMERSON; SISTLA, 1986), (HELJANKO, 1997) é que a busca nos sucessores de $s$ é completada mesmo quando a fórmula falha para um desses sucessores

${ }^{4}$ seguindo a semântica de $t_{q}$ mostrada no termo B na condição 3.16

${ }^{5}$ de acordo ao termo A da condição 3.16
} 
necesarios para atingir um estado onde $q$ é vardadeira. Se não estiver rotulado então retorna falso. Como nenhuma mudança foi feita neste caso pelo $D F S$ o conjunto das hipóteses $\{I\}$ é válida depois da execução de $D F S$.

Agora precisamos provar que o procedimento AUCheck garante que todos os estados sejam visitados pelo DFS.

Suponha que $G$ é a árvore gerada a partir do estado inicial do GTT usando a relação de sucessores de cada estado sem visitar estados que já foram visitados. Podemos garantir que esta árvore é finita devido a que o GTT é finito.

Durante a geração da árvore $G$ os seguintes casos podem ser encontrados:

Caso 1 a fórmula $\neg p \wedge \neg q$ é válida no estado $s$ passado como parâmetro ao procedimento DFS. O procedimento UpdateList coloca na variável List todos os sucessores de $s$ que não tenham sido visitados.

Caso 2 a fórmula $p \wedge \neg q$ é válida no estado $s$ passado como parâmetro ao procedimento DFS. Neste caso DFS é chamado de forma recursiva para cada sucessor de $s$.

Caso 3 a fórmula $q$ é válida no estado $s$ passado como parâmetro ao procedimento DFS. O procedimento UpdateList coloca na variável List todos os sucessores de $s$ que não tenham sido visitados.

Caso 4 o estado $s$ já foi visitado. Nada a ser feito.

Portanto, os sucessores de qualquer estado $s \in S$ sempre são visitados, seja através da sua colocação na Lista de estados a visitar ou seja pelo próprio $D F S$ chamado de forma recursiva. Como todos os sucessores do estado inicial serão visitados uma vez pelo procedimento DFS a árvore será construída usando todos os estados do GTT.

Como o estado está vazio antes da chamada de $A U C h e c k$, e como I8 é válida, depois da execução de DFS para todos os estados do GTT o mesmo estará vazio. Sendo assim a seguinte dedução pode ser feita a partir das hipóteses de indução, provadas válidas anteriormente:

(a) $\forall s\left[\right.$ state $[s]$.visited $\left.\rightarrow\left(\forall\left[p U_{\sim_{c}} q\right] \in L(s)\right) \rightarrow s \models \forall\left[p U_{\sim_{c}} q\right]\right]$. devido a I6 e I11.

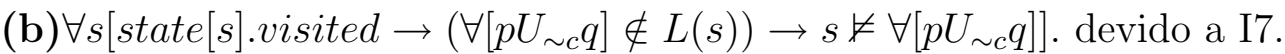

Então segue que: $\forall s\left[\right.$ state $[s]$.visited $\left.\rightarrow\left(\forall\left[p U_{\sim_{c}} q\right] \in L(s)\right) \leftrightarrow s \models \forall\left[p U_{\sim_{c}} q\right]\right]$.

Como na terminação de $A U C h e c k$ todos os estados foram visitados podemos garantir o seguinte:

$$
\forall s\left[\forall\left[p U_{\sim_{c}} q\right] \in L(s) \leftrightarrow s \models \forall\left[p U_{\sim_{c}} q\right]\right]
$$




\section{B.2 Prova da proposição 39}

Prova Dado um domínio $\Delta$ na sua forma canônica, suponha que o mesmo é representado usando a seguinte representação gráfica: os nós representam as variáveis do domínio $\Delta$ (transições habilitadas no domínio com um nó extra que representa o valor 0 ), o peso de cada arco direcionado entre dois nós representa a maxima diferença entre essas duas variáveis.

Como $\Delta$ é um domínio válido, ele é consistente, o que implica que não têm ciclos negativos. $\operatorname{Sup}_{\Delta}(x-y)$ é o peso do menor caminho que sai do nó $x$ e termina no nó $y$ no grafo que representa o domínio $\Delta$. Se $\Delta$ está na sua forma canônica então o peso do menor caminho que começa em $x$ e termina em $y$ é de fato $\Delta x y$ que é o peso de arco que liga esses dois nós no sentido $x y$.

Suponha que $a_{f}$ é uma atividade disparável no domínio $\Delta$. Como $a_{f}$ é uma atividade disparável então $\forall a \in \operatorname{enb}(M), a-a_{f} \geq 0$ o que é mesma coisa que $\forall a \in \operatorname{enb}(M), a_{f}-a \leq 0$.

Sendo assim, o grafo que representa o domínio $\Delta$ completado com o conjunto de arcos de $a_{f}$ a $a, \forall a \in e n b(M)$ com peso 0 é consistente, ou seja, não têm ciclos negativos.

Se disparamos a atividade $a_{f}$, seguindo a regra de disparo isto vai nos levar a uma nova marcação $M^{\prime}$ computada segundo dita regra e a um novo domínio temporal $\Delta^{\prime}$ que pode ser computado a partir do grafo do domínio anterior.

O novo grafo que representa o domínio $\Delta^{\prime}$ possui os nós $a_{n}$ que representam as atividades recém habilitadas pela nova marcação e os novos arcos $\left(a_{f}, a_{n},-\operatorname{tmin}\left(a_{n}\right)\right)$ e $\left(a_{n}, a_{f}, t \max \left(a_{n}\right)\right)$. O nó $a_{f}$ no domínio $\Delta$ passa a ser o nó o no domínio $\Delta^{\prime}$ devido a que todos os tempos no novo domínio são relativos ao tempo de disparo da atividade $a_{f}$.

Sendo assim, para todas as atividades habilitadas no novo domínio teremos:

$\Delta_{a o}^{\prime}$ é o peso do menor caminho entre o nó $a$ e o nó $a_{f}$. Se $a$ é uma atividade recém habilitada o menor caminho e único é o do arco $\left(a, a_{f}, t \max (a)\right)$ que foi adicionado ao novo domínio. Se $a$ é uma atividade disparável no domínio anterior então o peso do menor caminho é o caminho entre o nó $a$ e o no $a_{f}$ que corresponde a o nó o do novo domínio, portanto $\Delta_{a a_{f}}$.

$\Delta_{o a}^{\prime}$ é o peso do menor caminho entre o nó $a_{f}$ e o nó $a$. No caso em que $a$ é uma atividade recém habilitada o menor caminho e único é o do arco $\left(a_{f}, a,-t \min (a)\right)$ que foi adicionado ao novo domínio. Se $a$ é uma atividade habilitada no domínio anterior o peso no grafo do domínio anterior não pode ser mas usado pois a máxima diferença entre estas variáveis era devido ao menor valor de disparo de $a$ e o maior valor de disparo de $a_{f}$ e o disparo de $a_{f}$ aconteceu entre o menor valor da própria $a_{f}$ e o menor valor entre os $\operatorname{arcos}\left(\right.$ ao) do domínio anterior. Portanto o menor caminho entre o nó $a_{f}$ e o nó $a$ têm de ser computado sem levar em consideração o caminho direto entre estes. Sendo assim, o menor caminho seria o menor peso entre os caminhos dos nós correspondentes as atividades $u$ habilitadas no domínio anterior que terminam no nó $a$ devido a introdução dos $\operatorname{arcos}\left(a_{f}, u, 0\right)$. Portanto $\Delta_{o a}^{\prime}=\operatorname{Min}_{u \in \Upsilon_{s}} \Delta_{u a}$. 
$\Delta_{a a^{\prime}}^{\prime}$ é o peso do menor caminho entre os nós $a$ e $a^{\prime}$ habilitados pela nova marcação $M^{\prime}$. Se $a$ e $a^{\prime}$ são as mesmas obviamente a diferença entre eles é 0 . Se alguma delas é uma atividade recém habilitada então a nova diferença é computada segundo a definição a qual estabelece que a maior diferença acontece quando $a$ têm seu menor valor e $a^{\prime}$ seu maior valor, o menor valor de $a$ no domínio $\Delta^{\prime}$ é representado pelo arco (ao) e o maior pelo arco (oa') então $\Delta_{a a^{\prime}}^{\prime}=\Delta_{a o}^{\prime}+\Delta_{o a^{\prime}}^{\prime}$. No caso que as atividades $a$ e $a^{\prime}$ estejam habilitadas desde o domínio anterior então a máxima diferença entre elas será a própria diferença naquele domínio, ou seja $\Delta_{a a^{\prime}}$, ou um novo caminho formado através do novo nó o com os arcos (ao) e (oa'), ou seja, $\Delta_{a o}^{\prime}+\Delta_{o a^{\prime}}^{\prime}$. Portanto $\Delta_{a a^{\prime}}^{\prime}=\operatorname{Min}\left(\Delta_{a a^{\prime}}, \Delta_{a o}^{\prime}+\Delta_{o a^{\prime}}^{\prime}\right)$

Note-se que o tempo máximo de uma atividade recém habilitada é o $L F T$ do intervalo ligado a atividade mais o maior tempo mínimo de habilitação dessa atividade no estado atual, ou seja, $\operatorname{tmax}(a)=\uparrow a+\uparrow \nu_{s^{\prime}}(a)$ e o tempo mínimo de uma atividade recém habilitada é o EFT do intervalo ligado a atividade mais o menor tempo mínimo de habilitação dessa atividade no estado atual, ou seja, $\operatorname{tmin}(a)=\downarrow a+\downarrow \nu_{s^{\prime}}(a)$.

\footnotetext{
${ }^{6}$ lembre que a diferença do arco (oa') foi recalculada
} 


\section{Anexo A - Complexidade de Algoritmos}

O termo complexidade ligado ao problema de verificação como tal refere-se a complexidade computacional do problema de verificação. Entende-se por complexidade a relação de crescimento dos recursos a serem usados para resolver o problema com relação ao tamanho dos dados de entrada, expressa em número de bits. Os recursos podem ser tempo de uso da $C P U$, espaço de memória, consumo de energia, entre outros (WANG, 2004).

Alguns dos termos usados para se referir às classes de complexidades e seus respectivos significados aparecem listados na tabela A.1. Estes termos serão usados ao longo deste trabalho para caracterizar a complexidade dos diferentes problemas e algoritmos a que se fazem referência.

\begin{tabular}{|c|l|}
\hline Abreviatura & Significado \\
\hline PTIME & $\begin{array}{l}\text { Problemas que consomem tempo polinomial com relação } \\
\text { ao tamanho da entrada em número de bits. }\end{array}$ \\
\hline EXPTIME & $\begin{array}{l}\text { Problemas que consomem tempo exponencial com } \\
\text { relação ao tamanho da entrada em número de bits. }\end{array}$ \\
\hline PSPACE & $\begin{array}{l}\text { Problemas que consomem capacidade de memória poli- } \\
\text { nomial com relação ao tamanho da entrada em número } \\
\text { de bits. O tempo de resolução destes problemas é ilimi- } \\
\text { tado. }\end{array}$ \\
\hline EXPSPACE & $\begin{array}{l}\text { Problemas que consomem capacidade de memória expo- } \\
\text { nencial com relação ao tamanho da entrada em número } \\
\text { de bits. Geralmente estes problemas são mais difíceis } \\
\text { que os EXPTIME pois o tempo para resolve-los é ilimi- } \\
\text { tado. }\end{array}$ \\
\hline NP & $\begin{array}{l}\text { Problemas para os quais não se pode obter uma solução } \\
\text { em tempo polinomial com relação ao tamanho da en- } \\
\text { trada. }\end{array}$ \\
\hline NP-completos & $\begin{array}{l}\text { Conjunto de problemas mais difíceis da classe NP os } \\
\text { quais em geral são considerados intratáveis. }\end{array}$ \\
\hline indecidíveis & $\begin{array}{l}\text { Conjunto de problemas para os quais não há terminação } \\
\text { garantida. Geralmente não é possível projetar algorit- } \\
\text { mos para este tipo de problema. }\end{array}$ \\
\hline
\end{tabular}

Tabela A.1: Algumas classes de complexidades presentes em problemas de verificação. 


\section{Anexo B - Complexidade de Algoritmos usados em Verificadores de Modelos CTL}

Heljanko (1997) fez uma comparação na complexidade de diferentes algoritmos usados para verificação de modelos. Os algoritmos incluídos neste trabalho foram os seguintes:

EMC: é um algoritmo para verificação de modelos global para a lógica CTL proposto em (CLARKE; EMERSON; SISTLA, 1986).

Naive: é também um algoritmo de verificação global para a lógica CTL proposto em (HELJANKO, 1997).

ALMC: é um algoritmo de verificação local para a lógica CTL proposto em (VERGAUWEN; LEWI, 1993).

New: é um algoritmo que pode realizar verificação tanto global como local da lógica CTL proposto em (HELJANKO, 1997).

A tabela B.1 mostra os piores cenários de tempo de execução e requerimento de espaço de memória destes algoritmos. Nas tabelas, a notação $|\phi|$ foi usada em lugar de length $(\phi)$ para denotar o comprimento da fórmula. Também usamos $|S|,|R|$ no lugar de $\operatorname{card}(S), \operatorname{card}(R)$ respectivamente.

\begin{tabular}{|l|c|c|}
\hline Algoritmo & Tempo & Espaço \\
\hline Naive & $O(|\phi| \times|S| \times(|S|+|E|))$ & $O(|\phi| \times|S|+|E|)$ \\
\hline EMC & $O(|\phi| \times(|S|+|E|))$ & $O(|\phi| \times|S|+|E|)$ \\
\hline ALMC & $O(|\phi| \times(|S|+|E|))$ & $O(|\phi| \times|S|+|E|)$ \\
\hline New & $O(|\phi| \times(|S|+|E|))$ & $O(|\phi| \times|S|+|E|)$ \\
\hline
\end{tabular}

Tabela B.1: Tempo de execução e requerimento de espaço de memória para algumas implementações de verificadores de modelos

A tabelas B.2 e B.3 mostram de forma mais detalhada o requerimento de espaço de memória dos algoritmos de verificação abordados em (HELJANKO, 1997). 
156 Anexo B - Complexidade de Algoritmos usados em Verificadores de Modelos CTL

\begin{tabular}{|l|l|l|l|}
\hline Algoritmo & Mapa de bits & Busca em profundidade & Consumo de stack \\
\hline Naive & $|S| \times|\phi|$ & - & - \\
\hline EMC & $|S| \times(|\phi|+1)$ & - & $O(|S| \times\lceil\log |S|\rceil)$ \\
\hline ALMC & $|S| \times|\phi| \times 2$ & $|S| \times\lceil\log |S|\rceil$ & $O(|S| \times\lceil\log |S|\rceil \times|\phi|)$ \\
\hline New & $|S| \times(|\phi|+2)$ & $|S| \times\lceil\log |S|\rceil$ & $O(|S| \times\lceil\log |S|\rceil)$ \\
\hline
\end{tabular}

Tabela B.2: Requerimento de espaço de memória em bits para verificação da lógica CTL

\begin{tabular}{|l|l|}
\hline Algoritmo & Sucessores e predecessores \\
\hline Naive & $|R| \times\lceil\log |S|\rceil+|S| \times\lceil\log |R|\rceil$ \\
\hline EMC & $(|R| \times\lceil\log |S|\rceil+|S| \times\lceil\log |R|\rceil) \times 2$ \\
\hline ALMC & $|R| \times\lceil\log |S|\rceil+|S| \times\lceil\log |R|\rceil$ \\
\hline New & $|R| \times\lceil\log |S|\rceil+|S| \times\lceil\log |R|\rceil$ \\
\hline
\end{tabular}

Tabela B.3: Requerimento de espaço de memória em bits para armazenar a estrutura do grafo 


\section{Anexo C - Modelagem do Sistema de Evaporação usando a Ferramenta UPPAAL}

Neste anexo é apresentada a modelagem do sistema de evaporação feito usando a ferramenta UPPAAL. Os modelos aqui apresentados foram gentilmente cedidos pelo Dr. José Mendes Machado da Universidade de Minho, Portugal e correspondem à modelagem feita no artigo (MACHADO et al., 2007) para o sistema de evaporação descrito no seção 5.2.

Na modelagem da planta foram considerados oito módulos: Tank1, Tank2, Heater, Mixer, Alarm, Steam, Condenser e Liquid. As figuras C.1 e C.2 mostram os autômatos correspondentes a cada um destes módulos.
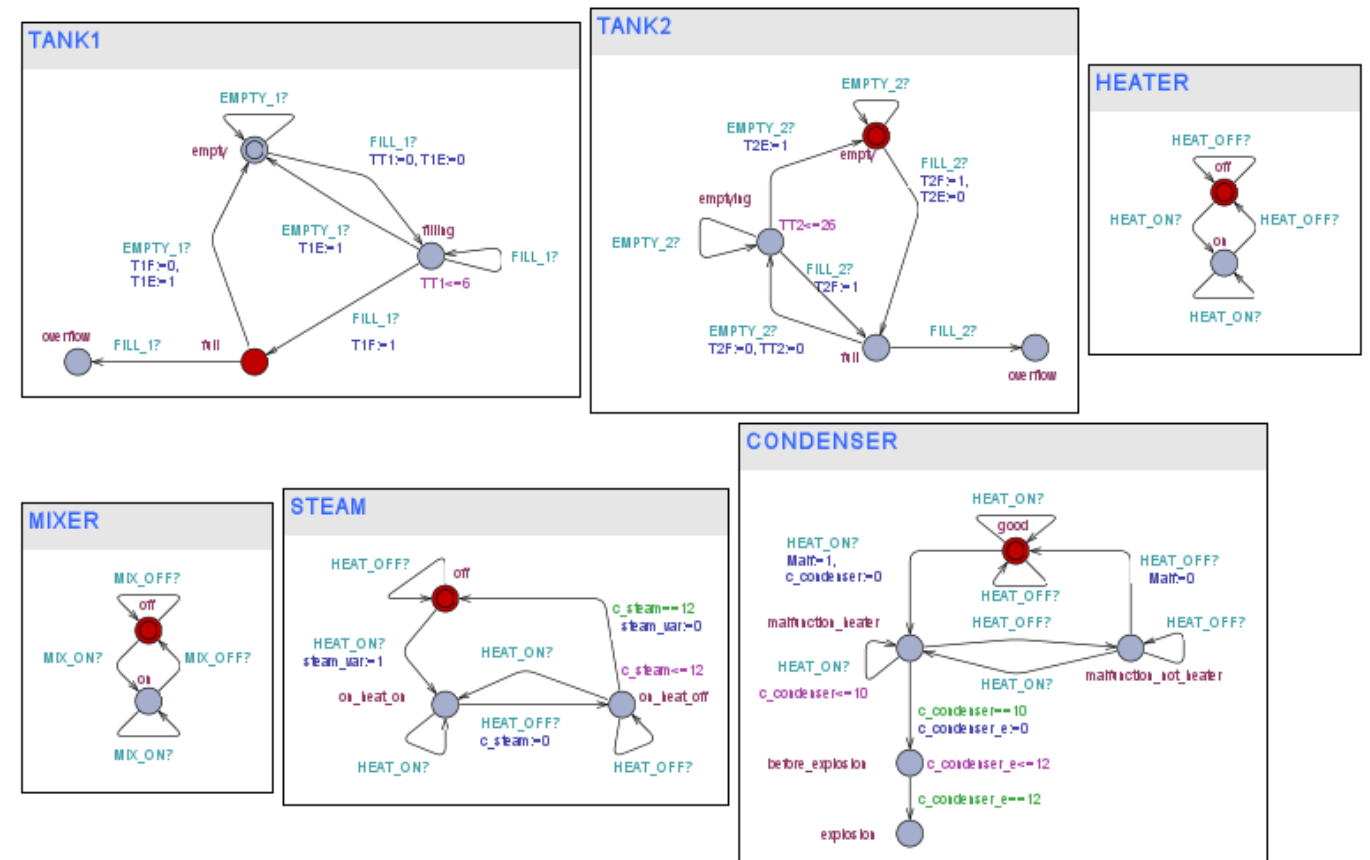

Figura C.1: Modelo em UPPAAL dos módulos Tank1, Tank2, Heater, Mixer, Steam e Condenser.

Os módulos Ton_3, Ton_4 e Ton_6 foram usados como temporizadores. Estes módulos são necessários para computar as ações de controle que dependem da contagem de um tempo determinado como ligar o misturador, ligar o aquecedor e esvaziar o tanque 2 . 


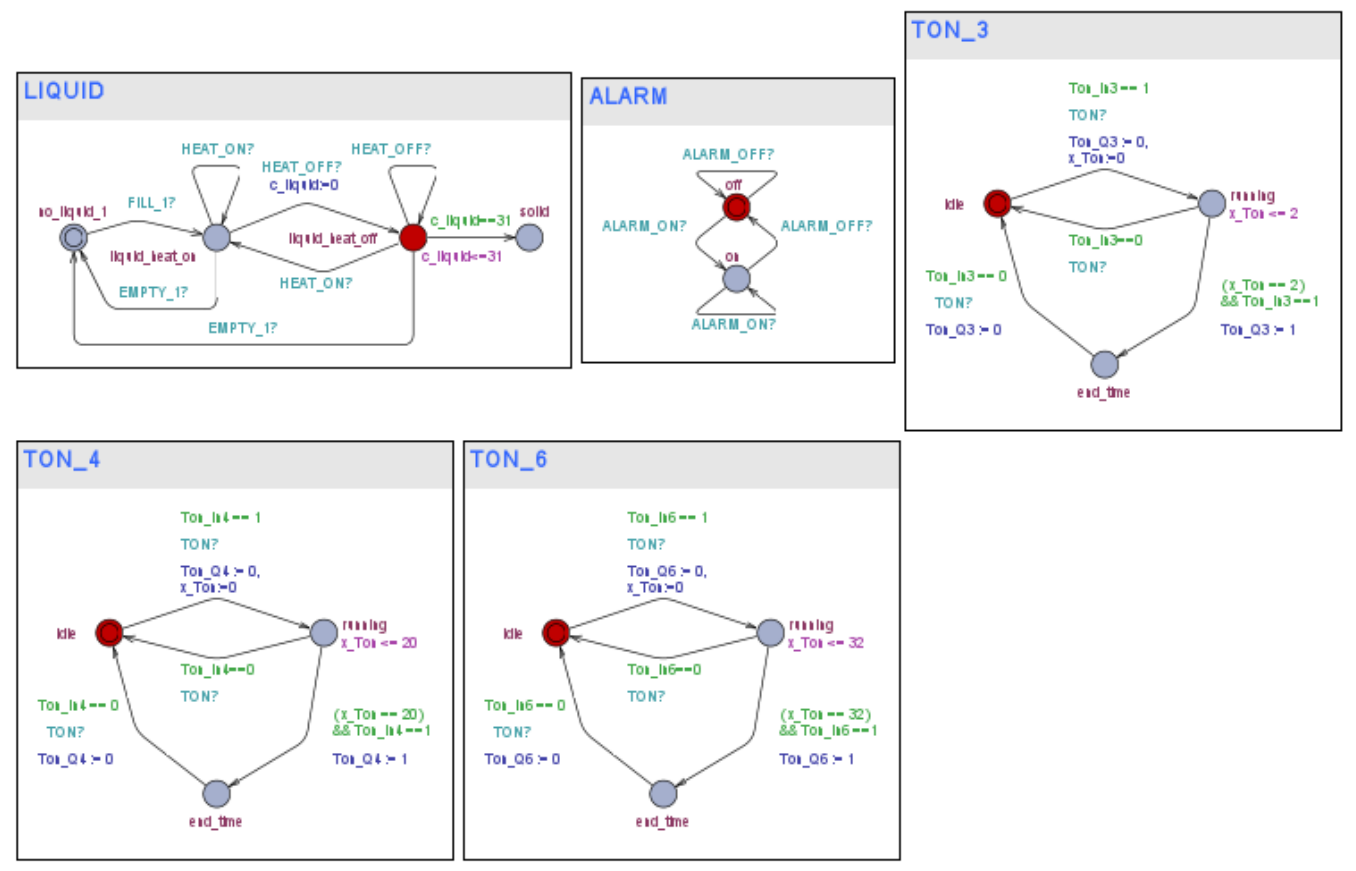

Figura C.2: Modelo em UPPAAL dos módulos Liquid, Alarm, Ton_3, Ton_4 e Ton_6.

O controlador para o sistema de evaporação foi projetado usando o método proposto em (SEABRA et al., 2007) partindo da especificação em SFC (a norma IEC 60848) a qual é apresentada na figura C.3.

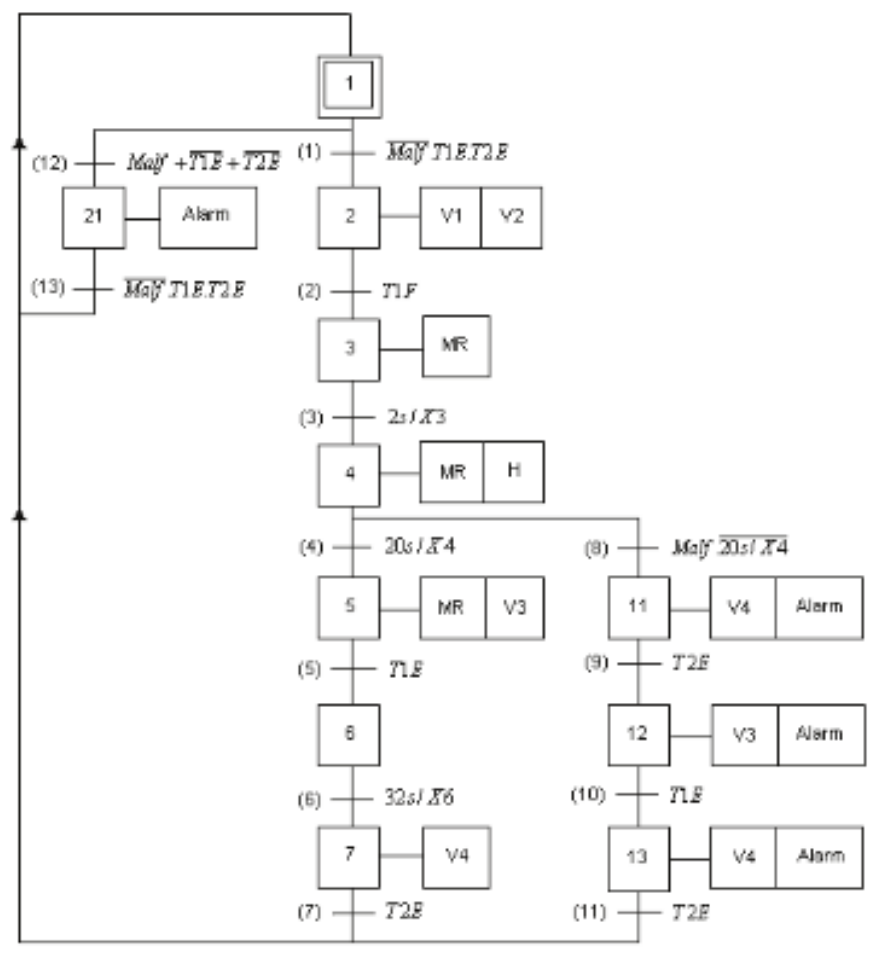

Figura C.3: Especificação em SFC do controlador do Sistema de Evaporação. 
A figura C.4 mostra o módulo Ladder que representa o funcionamento do controlador do sistema de Evaporação.

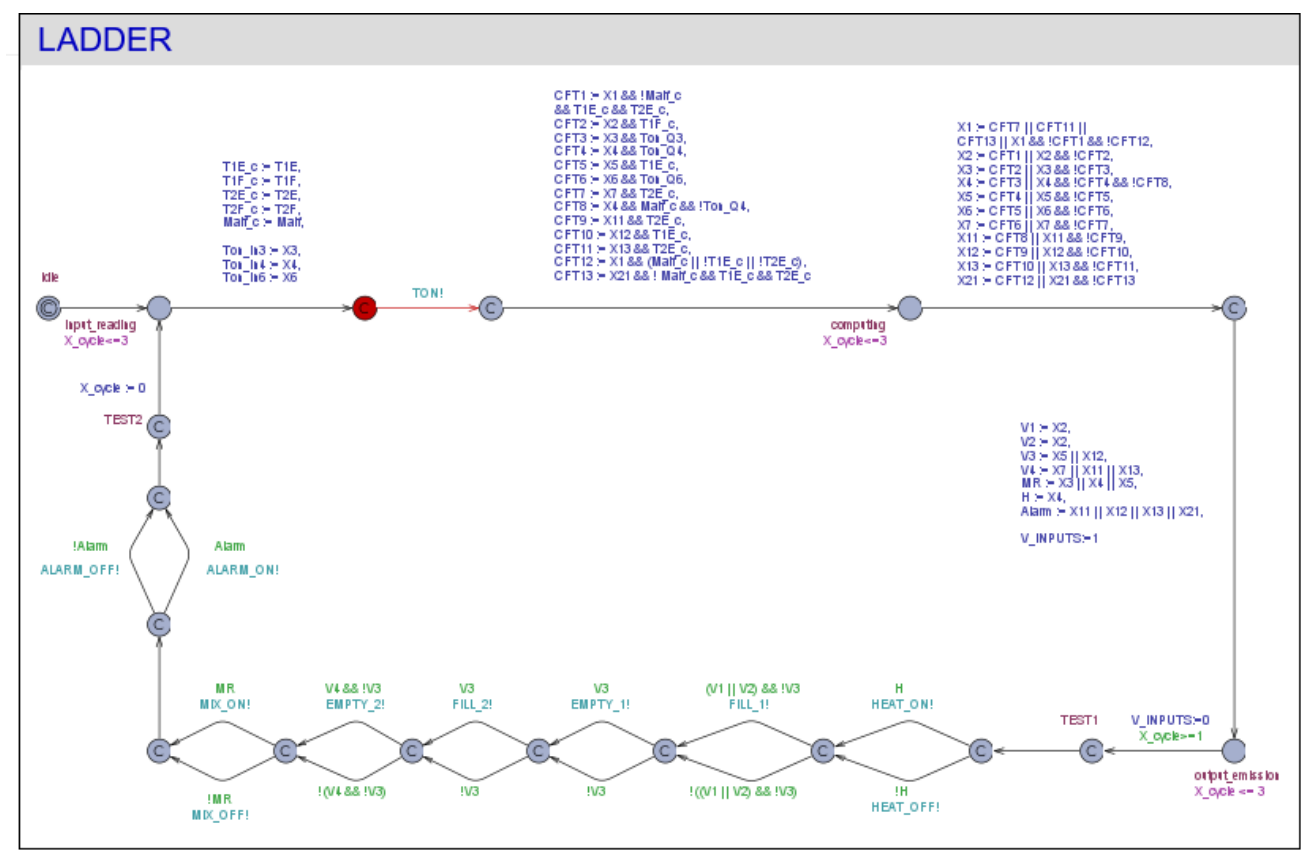

Figura C.4: Modelo em UPPAAL do módulo Ladder.

Note que o tempo previsto para a realização do ciclo do CLP é um valor entre 1 e 6 unidades de tempo o que resulta irreal dado os valores de tempo estabelecidos para o sistema de evaporação. A determinação das ações a serem realizadas em cada ciclo do CLP é feito através da determinação das clearing conditions e logo as variáveis de saída são atualizadas levando em consideração estes resultados.

Finalmente a figura C.5 mostra os resultados da verificação de algumas das propriedades verificadas em (MACHADO et al., 2007) e outras inseridas pelo autor deste trabalho.

Das propriedades verificadas, as primeiras 4 propriedades são propriedades de segurança, as dois seguintes são propriedades de atingibilidade e a última verifica a ausência de bloqueios no sistema. Os tempos envolvidos na verificação de cada uma destas propriedades não ultrapasso os 2 segundos em nenhum dos casos. 


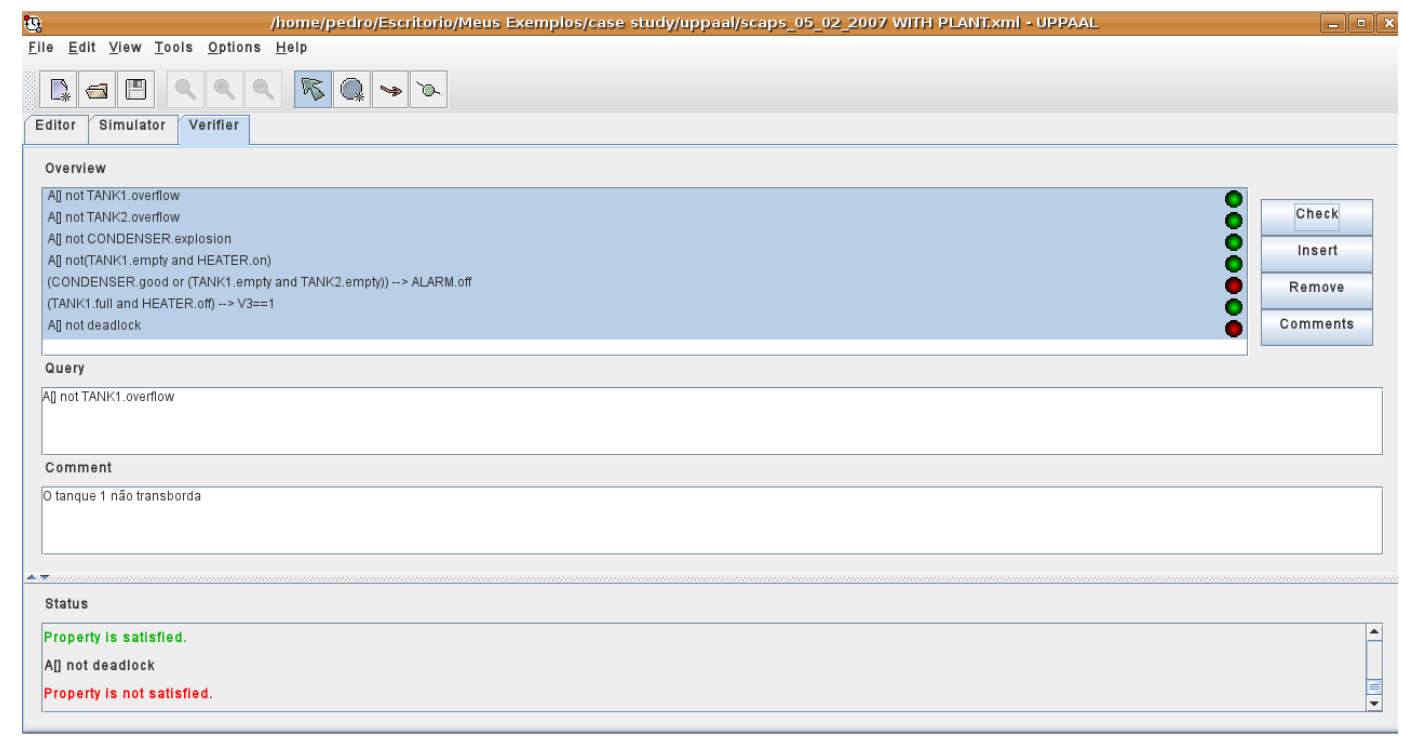

Figura C.5: Resultado da verificação para o Sistema de Evaporação. 